Florida International University FIU Digital Commons

$11-4-2016$

\title{
The Optimization of Pressure Cycling Technology (PCT) for Differential Extraction of Sexual Assault Casework
}

Vanessa Martinez

vmart011@fiu.edu

DOI: $10.25148 /$ etd.FIDC001223

Follow this and additional works at: https://digitalcommons.fiu.edu/etd

Part of the Analytical Chemistry Commons, Biotechnology Commons, and the Forensic Science and Technology Commons

\section{Recommended Citation}

Martinez, Vanessa, "The Optimization of Pressure Cycling Technology (PCT) for Differential Extraction of Sexual Assault Casework" (2016). FIU Electronic Theses and Dissertations. 2999.

https://digitalcommons.fiu.edu/etd/2999 


\section{FLORIDA INTERNATIONAL UNIVERSITY}

Miami, Florida

THE OPTIMIZATION OF PRESSURE CYCLING TECHNOLOGY (PCT) FOR DIFFERENTIAL EXTRACTION OF SEXUAL ASSAULT CASEWORK

A thesis submitted in partial fulfillment of

the requirements for the degree of

MASTER OF SCIENCE

in

CHEMISTRY

by

Vanessa Martinez

2016 
To: Dean Michael R. Heithaus

College of Arts, Sciences and Education

This thesis, written by Vanessa Martinez, and entitled The Optimization of Pressure Cycling Technology (PCT) for Differential Extraction of Sexual Assault Casework, having been approved in respect to style and intellectual content, is referred to you for judgment.

We have read this thesis and recommend that it be approved.

Martin Tracey

Francisco Fernandez-Lima

Bruce McCord, Major Professor

Date of Defense: November 4, 2016

The thesis of Vanessa Martinez is approved.

Dean Michael R. Heithaus

College of Arts, Sciences and Education

Andrés G. Gil

Vice President for Research and Economic Development and Dean of the University Graduate School

Florida International University, 2016 
C Copyright 2016 by Vanessa Martinez

All rights reserved. 


\section{DEDICATION}

I dedicate this thesis to the memory of my mother, Marisol Martinez, who supported me through every stage of my life and education. I miss you Mom. 


\section{ACKNOWLEDGMENTS}

First and foremost, I would like to express my gratitude to my major professor, Dr. Bruce McCord. I could not have hoped for a better mentor. Everything I have accomplished at this institution is thanks to his guidance and support. I would like to thank my committee members Dr. Martin Tracey and Dr. Francisco Fernandez-Lima for their time and efforts in helping me with this endeavor. I must also express my appreciation to Dr. Watson Lees for all his help in keeping me on the path to graduation and to Magali Autie for her invaluable assistance.

I must also acknowledge my fellow graduate students especially Meghan Roig and Fabiana Taglia, who were always there to lend a helping hand or to give encouragement. My thanks go to our lab manager Joana Antunes who has done a wonderful job in her role and to Karin Crenshaw for providing me with information necessary for the completion of my research.

Finally, I would like to thank Dr. Nathan Lawrence and Pressure BioSciences, Inc. for their continuing funding and support. 


\title{
ABSTRACT OF THE THESIS \\ THE OPTIMIZATION OF PRESSURE CYCLING TECHNOLOGY (PCT) FOR DIFFERENTIAL EXTRACTION OF SEXUAL ASSAULT CASEWORK
}

\author{
by \\ Vanessa Martinez \\ Florida International University, 2016 \\ Miami, Florida \\ Professor Bruce McCord, Major Professor
}

\begin{abstract}
A two-step protocol has been devised as a rapid and selective alternative to conventional differential extraction techniques with an increased recovery of DNA. The protocol involves pressure cycling with the Barocycler ${ }^{\circledR}$ NEP 2320 from Pressure Biosciences. Inc. in alkaline conditions for epithelial cell lysis and removal. This step is followed by alkaline lysis at $95^{\circ} \mathrm{C}$ for extraction of sperm cell DNA. At 1:1 or 2:1 female to male cell ratios, high selectivity and complete separation can be achieved. But at higher ratios, male allelic dropout is observed. This protocol has been modified to generate a clean male profile at a 20:1 cell ratio through optimization of $\mathrm{NaOH}$ concentration and inclusion of an additional pressure cycling step. Validation studies have been performed to assess the efficiency of this method under various conditions. An additional immunomagnetic cell capture pretreatment allowed for nearly complete separation at cell ratios of up to 200:1.
\end{abstract}




\section{TABLE OF CONTENTS}

\section{CHAPTER}

PAGE

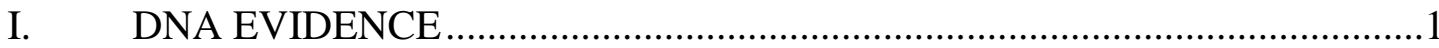

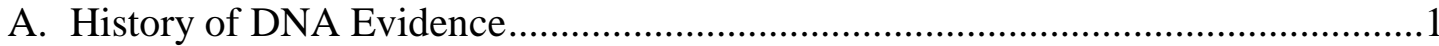

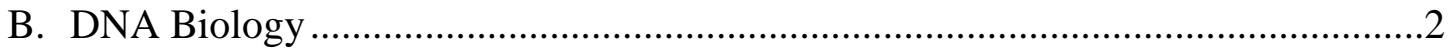

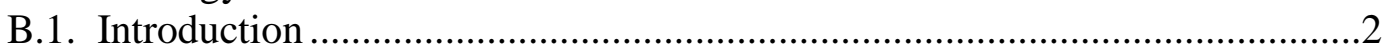

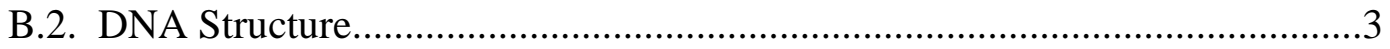

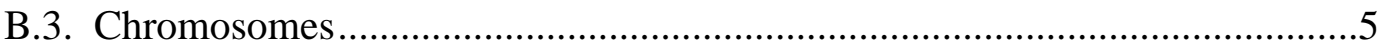

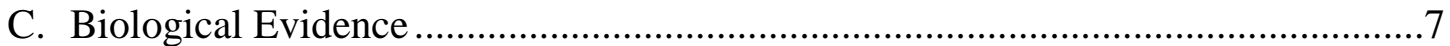

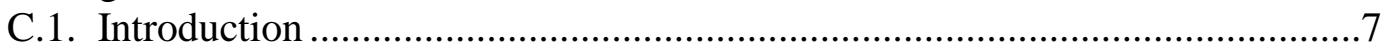

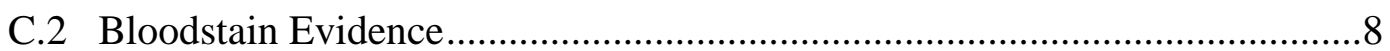

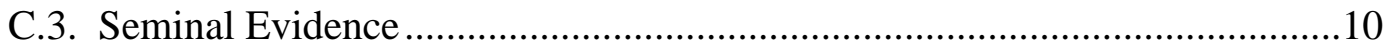

C.4 Epithelial Cells and Touch DNA …………..........................................11

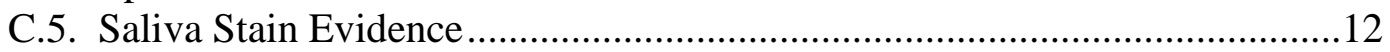

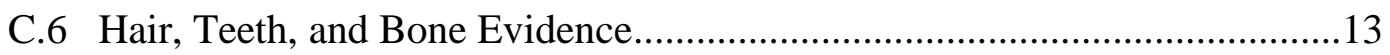

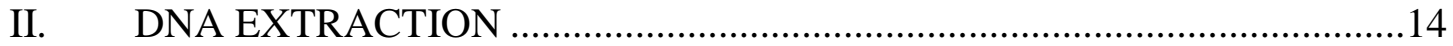

A. Extraction Methods …………………......................................................14

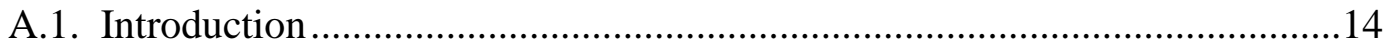

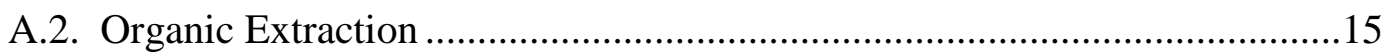

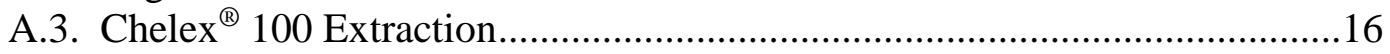

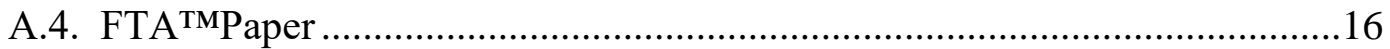

A.5. Solid-Phase Extraction ............................................................................

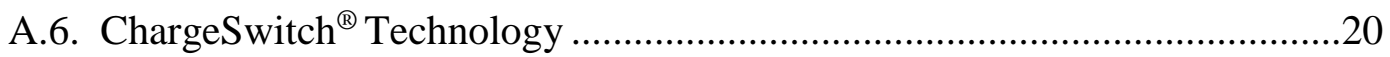

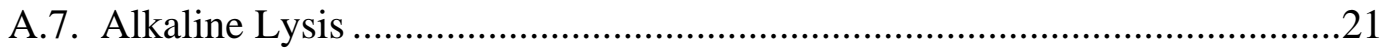

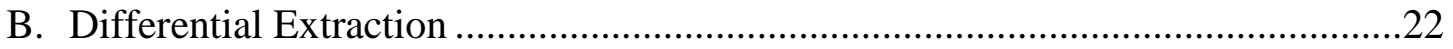

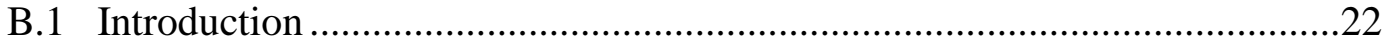

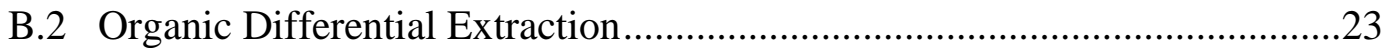

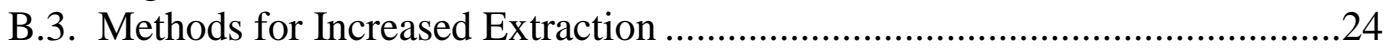

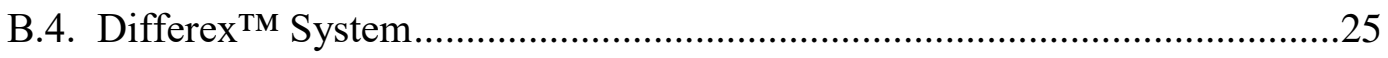

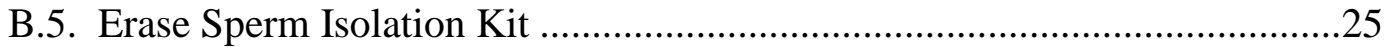

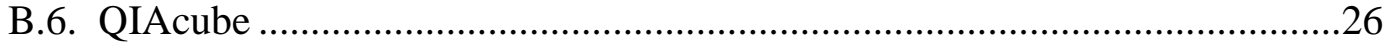

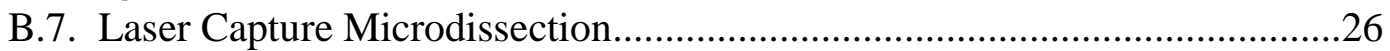

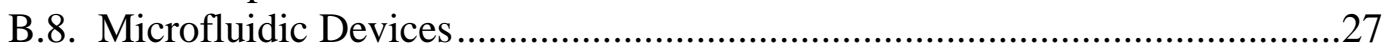

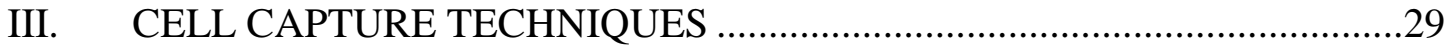

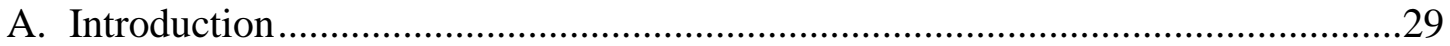

B. Sperm Cell Capture and Fertility Studies …………….......................................30

C. Sperm Cell Capture and Sexual Assault Evidence ………....................................30

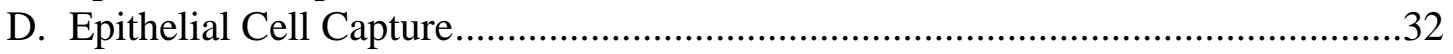

IV. PRESSURE CYCLING TECHNOLOGY (PCT) ...........................................33 


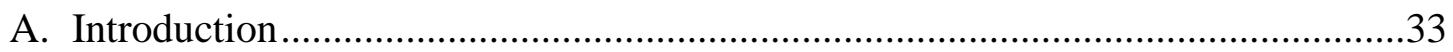

B. Barocycler ${ }^{\circledR}$ NEP 2320 Instrument and Components ...........................................34

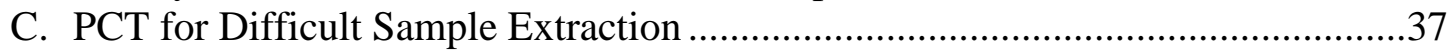

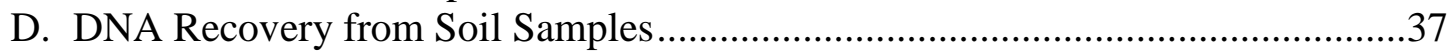

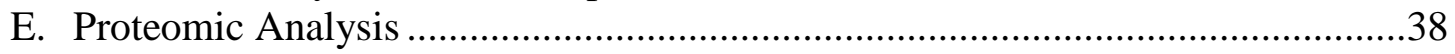

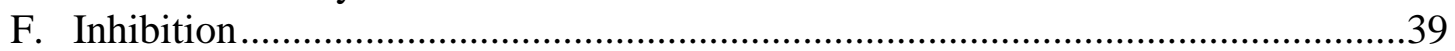

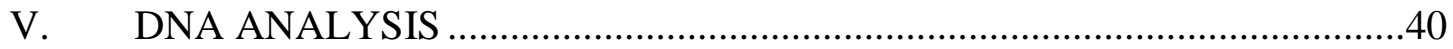

A. STR Analysis and Other Genetic Markers..........................................................40

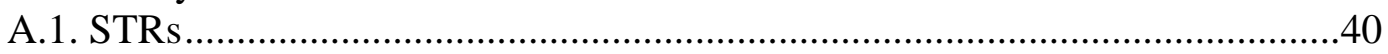

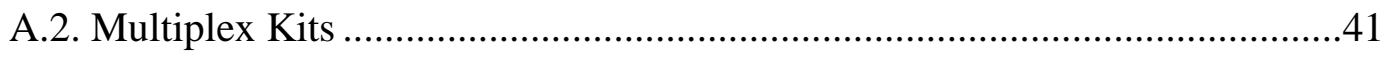

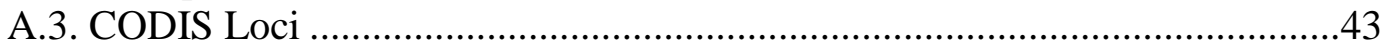

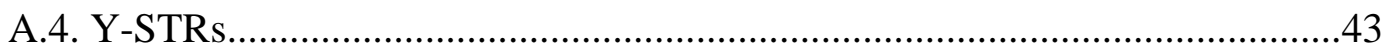

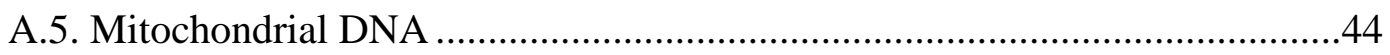

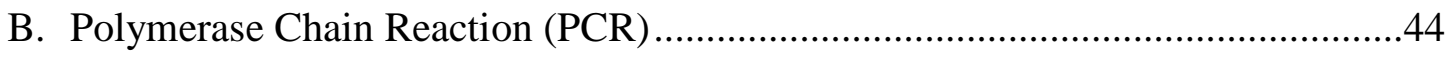

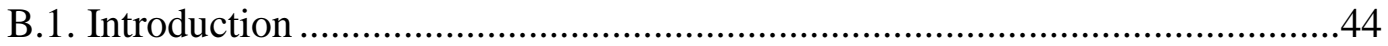

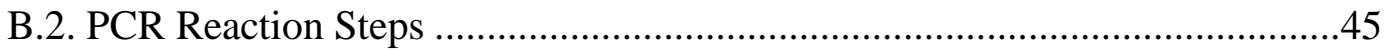

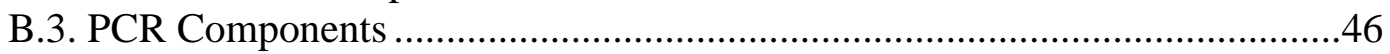

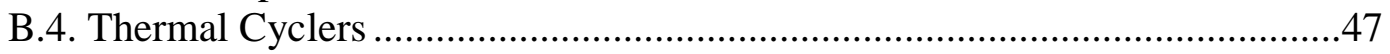

B.5. Real-time PCR

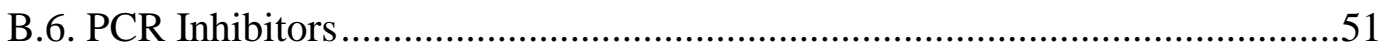

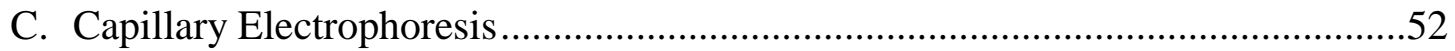

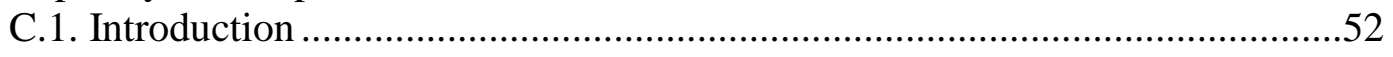

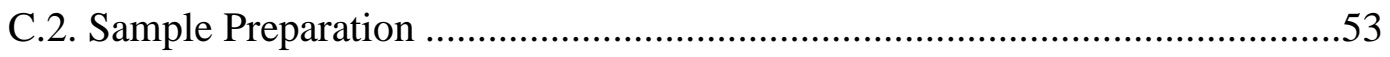

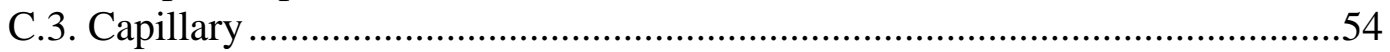

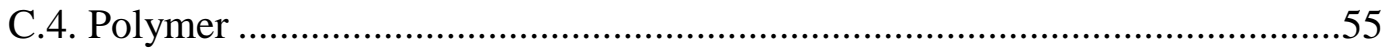

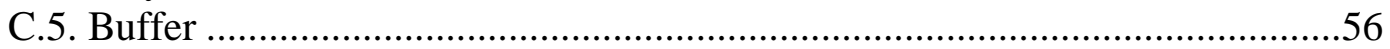

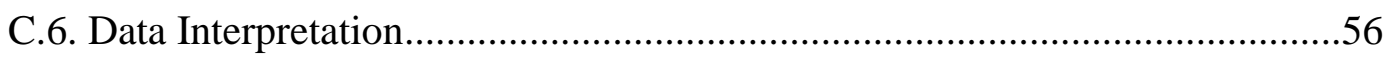

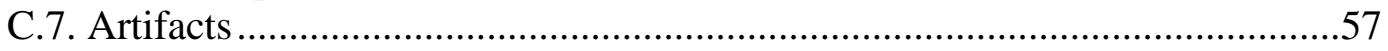

VI. OPTIMIZATION OF PRESSURE CYCLING AND ALKALINE LYSIS

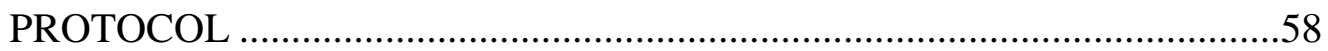

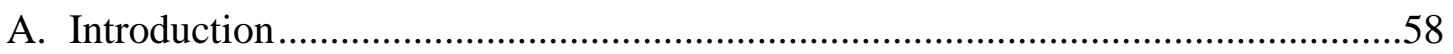

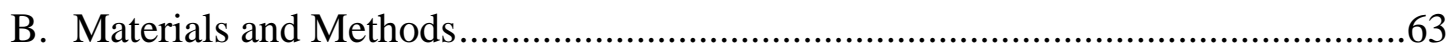

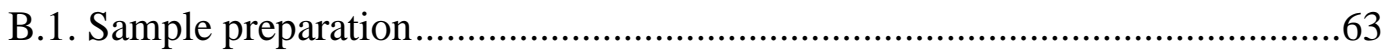

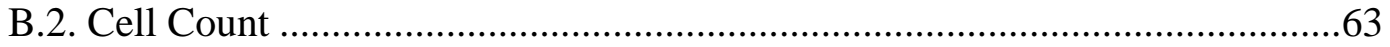

B.3. Pressure Cycling technology (PCT) Sample Preparation ...............................64

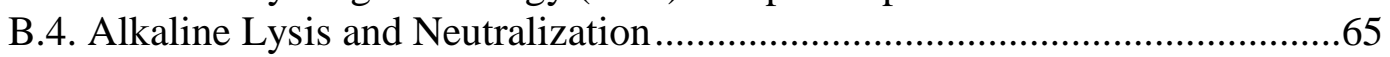

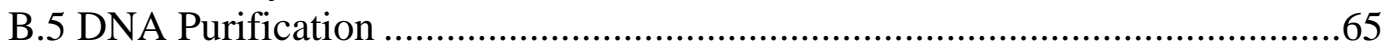

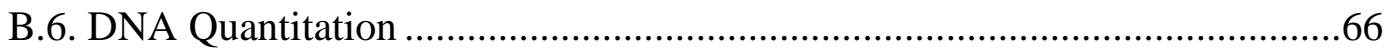

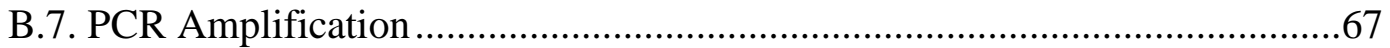

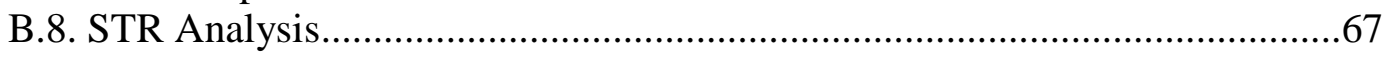

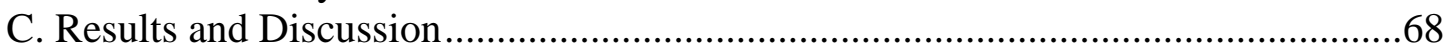

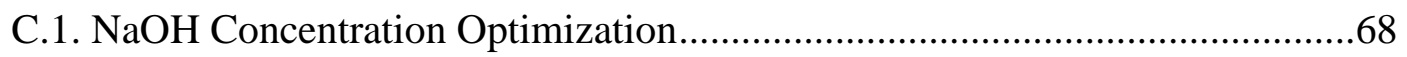

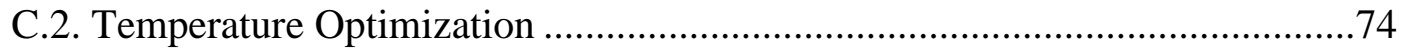




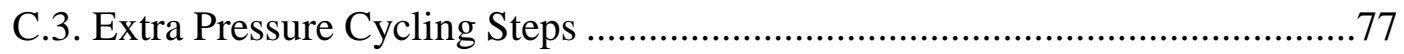

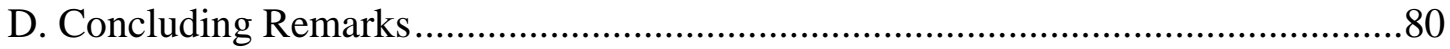

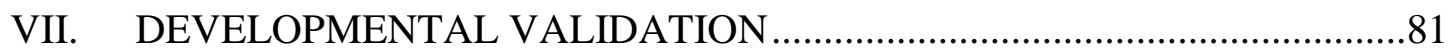

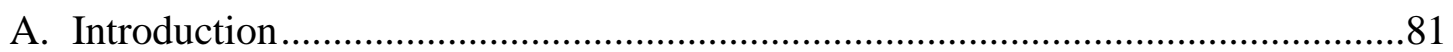

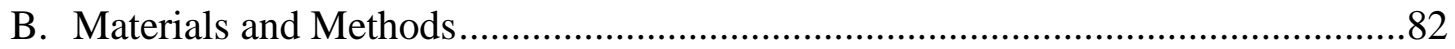

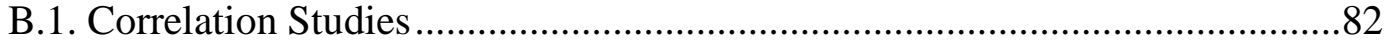

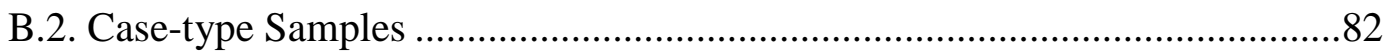

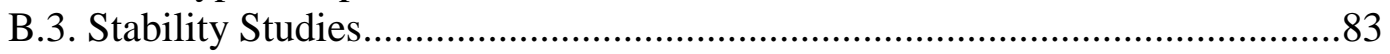

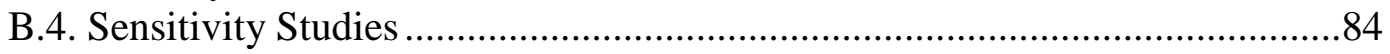

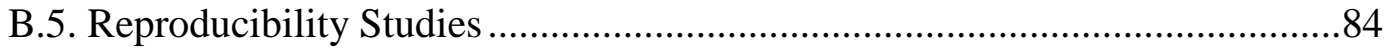

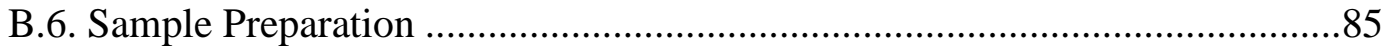

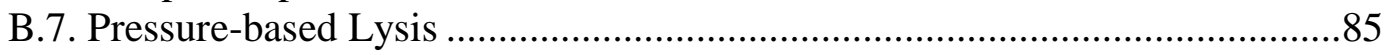

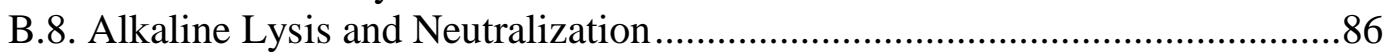

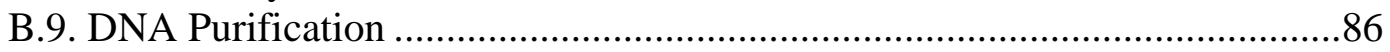

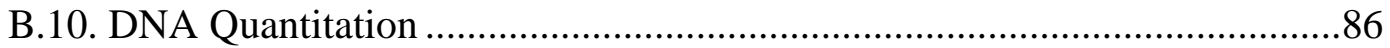

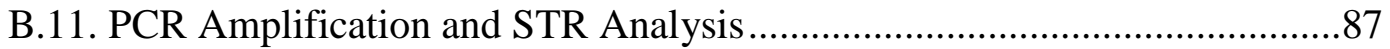

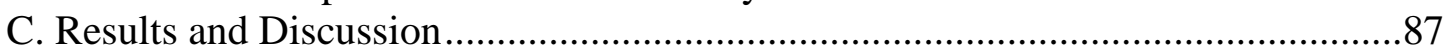

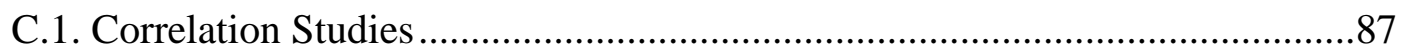

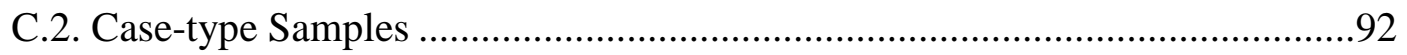

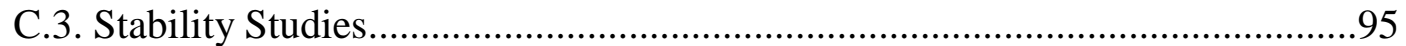

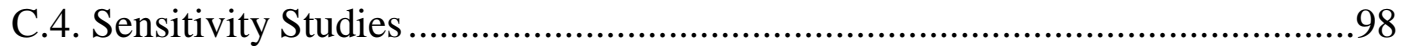

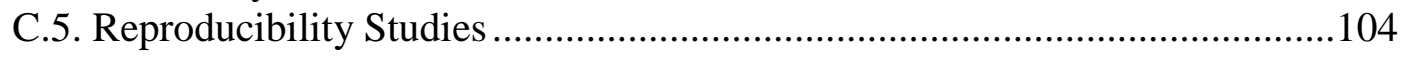

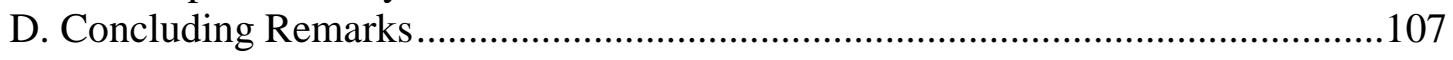

VIII. IMMUNOMAGNETIC CAPTURE (IMC) OF EPITHELIAL CELLS........109

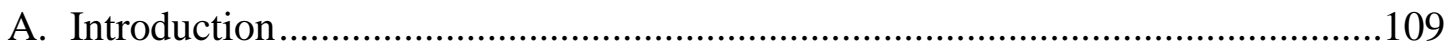

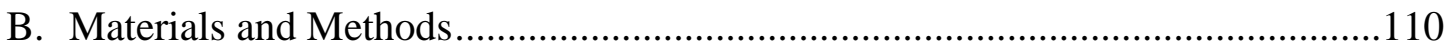

B.1. Cell Suspension Preparation......................................................................110

B.2. Cell Count and Sample Preparation .........................................................110

B.3. Immunomagnetic Capture (IMC) of Epithelial Cells.....................................111

B.4. Pressure Cycling Technology and Pressure-based Lysis ................................112

B.5. Alkaline Lysis and Neutralization .........................................................112

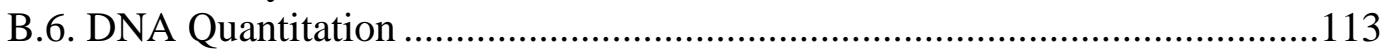

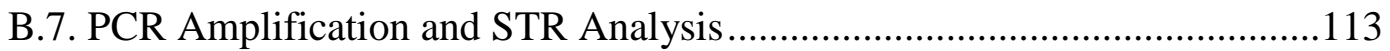

C. Results and Discussion.........................................................................113

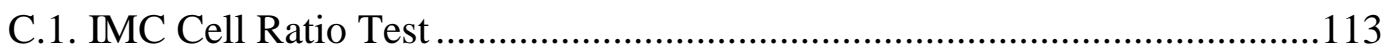

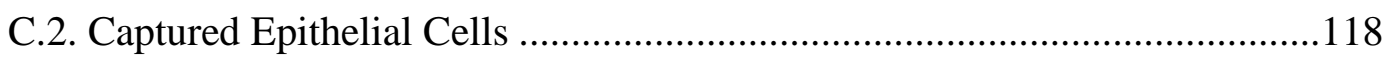

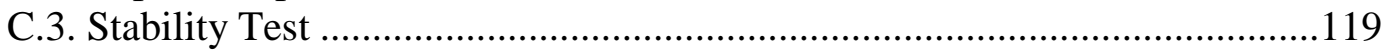

D. Concluding Remarks.................................................................................121

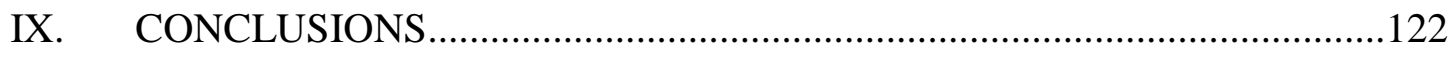

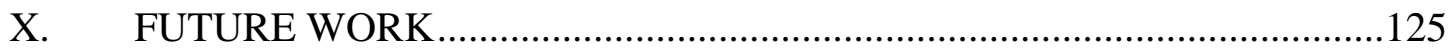


LIST OF REFERENCES. 


\section{LIST OF FIGURES}

FIGURE

PAGE

Figure 1. A DNA sequence is made up of 4 different nitrogenous bases; adenine, thymine, guanine and cytosine. Within the nucleus of each somatic cell, DNA is arranged into 23 pairs of chromosomes. Adapted from the National Institute of Health official

website.

Figure 2. The DNA backbone is made up of phosphate groups that connect deoxyribose molecules. Nitrogenous bases bonded to the deoxyribose molecules are paired with complementary bases on the other strand in an antiparallel fashion forming a double helix. Adapted from the U.S. National Library of Medicine website.............4

Figure 3. A healthy human male has a karyotype of 22 pairs of autosomes and an $X$ and Y chromosomes. Adapted from Broad Institute website

Figure 4. ABAcard $®$ HemaTrace $®$ for the Forensic Identification of Human Blood. Above is a positive result indicated with a pink line at the test region on the left as well as a positive result at the control region. Below is a negative result with a pink line at the control region only. Adapted from the Minnesota Bureau of Criminal Apprehension website.

Figure 5. A Christmas tree stain. Nucleic acids in sperm cell heads are stained red and tails are stained green for improved visibility. Adapted from St. Louis County Police Crime Laboratory website.

Figure 6. Whatman ${ }^{\circledR}$ FTA® ${ }^{\circledR}$ card technology from Sigma-Aldrich (St. Louis, MO). Adapted from Sigma-Aldrich website.

Figure 7. A general QIAamp procedure. Centrifugation (spin) or vacuum manifold may be employed. In either case the process involves, lysis, binding of DNA, washing, and elution. Adapted from Qiagen website. 
Figure 8. ChargeSwitch ${ }^{\circledR}$ purification. After sample lysis, the $\mathrm{pH}$ is altered to less than 6.0 for DNA binding to magnetic beads. The DNA bound to the beads is immobilized with a magnet and impurities are washed away. The $\mathrm{pH}$ is increased to 7.0 and the magnetic beads are washed. Increasing the $\mathrm{pH}$ to more than 8.5 releases the purified DNA. Adapted from ThermoFisher website

Figure 9. General organic differential extraction procedure. The first step involves incubation with the addition of detergent, buffer, and Proteinase $\mathrm{K}$ for epithelial cell lysis. After centrifugation, the supernatant is removed and the intact sperm pellet is left behind. Several wash steps often follow. Incubation with the addition of detergent, buffer, and DTT lyses the sperm cell releasing the DNA for subsequent purification and analysis.

Figure 10. A tetrameric antibody complex is formed bridging the EasySep ${ }^{\mathrm{TM}}$ magnetic particle and the EpCAM antigen present on the cell. The rat ant-mouse IgG monoclonal antibodies are pictures in yellow. The mouse monoclonal antibody that recognizes EpCAM is pictured in red. The mouse monoclonal antibody that attaches to the dextran-coated magnetic particle is pictured in blue. Adapted from Stemcell Technologies official website

Figure 11. Mechanism of pressure based lysis. (A) The cell membrane at ambient pressure. (B) The cell membrane compressed by the application of high pressure. (C) The cell membrane breaks apart when the high pressure is released and the cell is

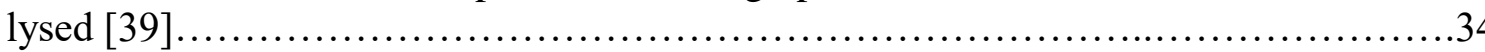

Figure 12. Barocycler® NEP 2320 from Pressure BioSciences, Inc. (South Easton, MA). Adapted from Pressure BioSciences, Inc. website ............................35

Figure 13. PULSE tube FT500. The ram transmits pressure to the sample and the screw cap seals the tube. The sample lies on top of the lysis disk filled with perforations that allow sample to be pushed into the collection chamber. Adapted for Pressure BioSciences, Inc. website.

Figure 14. Allelic ladder and internal size standard in the Y-STR STRtyper-27 system. The top four panels display the allelic ladder. The represented locus is 
labeled in the green bar above each panel and all common alleles are represented by the peaks below. The bottom panel displays the internal size standard labeled in an orange dye [8].

Figure 15. The PCR process includes three steps; denaturation, annealing, and extension. The cycle then repeats for exponential amplification. Adapted from ThermoFisher Scientific website.

Figure 16. Philisa® Thermal Cycler from Streck, Inc. (Nebraska, US). Adapted from Streck, Inc. website

Figure 17. Phases included in a qPCR reaction; exponential phase as product doubles each cycle, linear phase as reagents are depleted and reaction no longer proceeds exponentially, and the plateau phase is entered as regents are exhausted. Adapted from Abbot Molecular website...

Figure 18. As the extension step proceeds, the Dabcyl-iso-dGTP nucleotide is incorporated opposite the fluorescent reporter and fluorescence is quenched. Overall fluorescence in the reaction decreases as product increases. Adapted from Promega website.

Figure 19. 96-capillary 3730xl DNA Analyzer from ThermoFisher Scientific (Weston, FL). Adapted from ThermoFisher Scientific website

Figure 20. At $\mathrm{pH}>5$, the silanol groups become ionized creating an electric double layer composed of cations. An electroosmotic flow results in which all molecules are dragged toward the negatively charged cathode regardless of charge [37] ...............55

Figure 21. Flow chart depicting the developed PCT + alkaline lysis protocol. The chart details steps for obtaining separate epithelial and sperm fractions [75].

Figure 22. Percent contribution of male and female alleles in sperm fraction. Allelic peak heights from male and female contributors were divided by the total peak height at the respective locus. A total of seven loci with no shared alleles between the male 
and female DNA profiles were selected to calculate the percent contribution of male and female DNA [75]...

Figure 23. The effect of $\mathrm{NaOH}$ concentration on selectivity on 20:1 F:M samples. The highest male DNA contribution percentage was obtained at $0.05 \mathrm{~N}$ and the lowest at the original $0.4 \mathrm{~N}$ concentration. Percentage determined by male or female peak height divided by total peak height at 7 loci where no alleles are shared between the male or female contributor, $\mathrm{x} 100$. ( $\mathrm{n}=3 \pm$ standard error $)$.

Figure 24. The effect of $\mathrm{NaOH}$ concentration on female DNA recovery in the epithelial fraction obtained after the PCT step. The highest recovery occurred between $0.05 \mathrm{~N}$ and $0.2 \mathrm{~N}$. Higher and lower concentrations, $0.025 \mathrm{~N}$ and $0.4 \mathrm{~N}$,

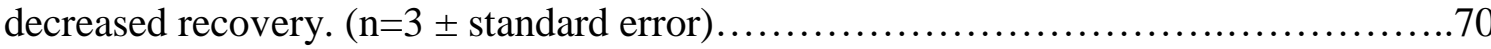

Figure 25. The effect of $\mathrm{NaOH}$ concentration on male DNA loss during the PCT step. As $\mathrm{NaOH}$ concentration increased, more male DNA was lost in the epithelial fraction during PCT. It is likely the result of the disruption of sperm cells by harsh alkaline conditions. ( $\mathrm{n}=3 \pm$ standard error)

Figure 26. Profile of the male contributor. Panel represents loci labeled with 5-TAMRA (5-Carboxytetramethylrhodamine); Amelogenin, D8S1179, TPOX, and FGA.......................................................................... 72

Figure 27. Profile of the female contributor. Panel represents loci labeled with 5-TAMRA (5-Carboxytetramethylrhodamine); Amelogenin, D8S1179, TPOX, and FGA.

Figure 28. Sperm fraction profile of a 20:1 F:M sample extracted with PCT + alkaline lysis protocol modified with $0.05 \mathrm{~N} \mathrm{NaOH}$ for the PCT step. A slight majority male profile is observed with a male DNA contribution of $68 \%$. Panel represents loci labeled with 5-TAMRA (5-Carboxytetramethylrhodamine); Amelogenin, D8S1179, TPOX, and FGA

Figure 29. Sperm fraction profile of a 20:1 F:M sample extracted with original PCT + alkaline lysis protocol with $0.4 \mathrm{~N} \mathrm{NaOH}$ for the PCT step. The percent contribution of male DNA in the profile was only $16 \%$. Panel represents loci labeled 
with 5-TAMRA (5-Carboxytetramethylrhodamine); Amelogenin, D8S1179, TPOX,

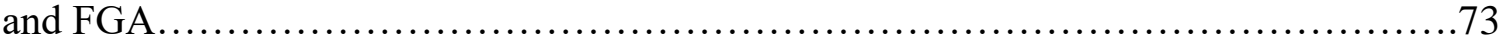

Figure 30. The effect of temperature during the PCT step on selectivity with 20:1 F:M samples. $25^{\circ} \mathrm{C}$ was the temperature that resulted in the highest male DNA contribution. Percentage determined by male or female peak height divided by total peak height at 7 loci where no alleles are shared between the male or female

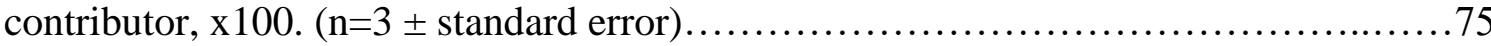

Figure 31. The effect of temperature on female autosomal DNA recovery in the epithelial fraction obtained after the PCT step. There is a slight trend for increasing recovery at higher temperature. ( $n=3 \pm$ standard error))

Figure 32. The effect of temperature on male DNA loss during the PCT step. At increased temperatures, more male DNA was lost in the epithelial during PCT is likely the result of the disturbance of sperm cells. ( $n=3 \pm$ standard error)

Figure 33. The effect of an additional PCT step on selectivity with 20:1 F:M samples. An increase in male DNA contribution resulted through lysis and recovery of residual epithelial cell DNA left behind in the swab. Percentage determined by male or female peak height divided by total peak height at 7 loci where no alleles are shared between the male or female contributor, $x 100(n=3 \pm$ standard error) ....................................... 78

Figure 34. Sperm fraction profile of a 20:1 F:M sample extracted with PCT + alkaline lysis protocol modified with $0.05 \mathrm{~N} \mathrm{NaOH}$ for the PCT step and an additional PCT step. A percent male DNA contribution of $87 \%$ is observed in the profile. Panel represents loci labeled with 5-TAMRA (5-Carboxytetramethylrhodamine); Amelogenin, D8S1 179, TPOX, and FGA ...................................... 79

Figure 35. The effect of an additional PCT step on selectivity with 20:1, 50:1, 100:1, and 200:1 F:M samples. Predictably, selectivity decreased with increasing amounts of epithelial cells. Majority male profiles were obtained at 50:1 and 100:1. Percentage determined by male or female peak height divided by total peak height at 7 loci where no alleles are shared between the male or female contributor, $\mathrm{x} 100$ 
Figure 36. Selectivity comparison between the PBSO and PCT + alkaline lysis method used to extract preserved post-coital samples. PBSO method gave superior results for most samples. Exceptions are observed for PC 158, PC 162, and PC 159. Determined through calculation of peak height ratio of $\mathrm{Y}$ over $\mathrm{X}$ at the Amelogenin locus.

Figure 37. Selectivity comparison between the PBSO and PCT + alkaline lysis method used to extract preserved post-coital samples. PBSO method showed greater male contribution but less reproducibility when compared to PCT + alkaline lysis. Determined through calculation of peak height ratio of $\mathrm{Y}$ over $\mathrm{X}$ at the Amelogenin locus.

Figure 38. Male DNA concentration in sperm fraction of post-coital samples after extraction with PBSO method or PCT + alkaline lysis method; PC 158, PC 159, PC 160, PC 162, PC 163, PC 177, and PC 177. Higher recovery of male DNA reported with samples PC 158 (373\% increase), PC 159 (342\% increase), PC 162 (4,847\% increase), and PC 163 (1,809\% increase)

Figure 39. Male DNA concentration in sperm fraction of post-coital samples after extraction with PBSO method or PCT + alkaline lysis method; PC 161, PC 176, and PC 180. Higher recovery of male DNA reported with samples PC 161 (49\% increase) and PC 180 (60\% increase)

Figure 40. Male DNA concentration in sperm fraction of post-coital sample PC 175 after extraction with PBSO method $(9.9 \pm 6.8 \mathrm{ng} / \mu \mathrm{L})$ or PCT + alkaline lysis method $(17 \pm 3.8 \mathrm{ng} / \mu \mathrm{L}) .(\mathrm{n}=3 \pm$ standard error $)$

Figure 41. Percent male DNA recovery in sperm fraction on extracted samples deposited on various substrates spiked with 5:1 F:M sample. Recovery was poor in all cases compared to the cotton swab. ( $\mathrm{n}=3 \pm$ standard error)

Figure 42. The effect of substrate on selectivity for the sperm fraction from a 5:1 F:M spiked sample. The cotton swab samples resulted in $89 \pm 0.77 \%$ male DNA contribution. Denim and cotton panties resulted in a slight majority female profile with a male DNA contribution of $43 \pm 2.8 \%$ and $43 \pm 2.5 \%$. Nearly all alleles present in the bedspread samples pertained to the male contributor with a male DNA 
contribution of $98 \pm 1.4 \%$. The percent male or female contribution was determined by the male or female peak height divided by total peak height at 7 loci where no alleles were shared between the male or female contributor, $x 100$. $(n=3 \pm$ standard error)

Figure 43. Figure 43. The effect of inhibitors and environmental insults on selectivity of 20:1 sample sperm fractions. Bile salts and tannic acid have a minimal effect on selectivity when compared to the control (20:1 F:M on a cotton swab). The percent male or female contribution was determined by the male or female peak height divided by total peak height at 7 loci where no alleles were shared between the male or female contributor, $\mathrm{x} 100$. ( $\mathrm{n}=3 \pm$ standard error).

Figure 44. Male DNA concentration in sperm fraction of 20:1 treated with either bile salts or tannic acid compared to a control. ( $n=3 \pm$ standard error)

Figure 45. The effect of environmental insults on the selectivity of 100:1 samples compared to control. The percent male or female contribution was determined by the male or female peak height divided by total peak height at 7 loci where no alleles were shared between the male or female contributor, $x 100$. ( $n=3 \pm$ standard error)

Figure 46. Male DNA concentration in the sperm fraction of 100:1 samples subjected to outdoor environmental conditions for 1 week compared to a control.

$(\mathrm{n}=3 \pm$ standard error).

Figure 47. Selectivity of developed protocol with decreasing number of cells present in sample. Male DNA contribution decreased with decreasing cell count. Percentage determined by male peak height divided by total peak height at 7 loci where no alleles are shared between the male or female contributor, $x 100$. ( $n=3 \pm$ standard error).

Figure 48. Sperm fraction profile of a 50,000:2,500 epithelial cell:sperm cell sample. A percent male DNA contribution of $90 \%$ is observed in the profile. Panel represents loci labeled with 5-TAMRA (5-Carboxytetramethylrhodamine); Amelogenin, D8S1179, TPOX, and FGA.... 
Figure 49. Sperm fraction profile of a 25,000:1,250 epithelial cell:sperm cell sample. A percent male DNA contribution of 55\% is observed in the profile. Panel represents loci labeled with 5-TAMRA (5-Carboxytetramethylrhodamine); Amelogenin, D8S1179, TPOX, and FGA

Figure 50. Sperm fraction profile of a 12,500:500 epithelial cell:sperm cell sample. A percent male DNA contribution of $36 \%$ is observed in the profile. Panel represents loci labeled with 5-TAMRA (5-Carboxytetramethylrhodamine); Amelogenin, D8S1179, TPOX, and FGA

Figure 51. Sperm fraction profile of a 6,250:250 epithelial cell:sperm cell sample. A percent male DNA contribution of $17 \%$ is observed in the profile. Panel represents loci labeled with 5-TAMRA (5-Carboxytetramethylrhodamine); Amelogenin, D8S1179, TPOX, and FGA.

Figure 52. Sperm fraction profile of a 3,125:125 epithelial cell:sperm cell sample. A percent male DNA contribution of $16 \%$ is observed in the profile. Panel represents loci labeled with 5-TAMRA (5-Carboxytetramethylrhodamine); Amelogenin, D8S1179, TPOX, and FGA....

Figure 53. Sperm fraction profile of a 375:75 epithelial cell:sperm cell sample.

A percent male DNA contribution of $66 \%$ is observed in the profile.

Dropout occurred at the FGA locus. Panel represents loci labeled with

5-TAMRA (5-Carboxytetramethylrhodamine); Amelogenin, D8S1179, TPOX, and

FGA

Figure 54. Sperm fraction profile of a 250:2,500 epithelial cell:sperm cell sample. A percent male DNA contribution of $94 \%$ is observed in the profile. Panel represents loci labeled with 5-TAMRA (5-Carboxytetramethylrhodamine); Amelogenin, D8S1179, TPOX, and FGA. .104

Figure 55. Reproducibility of male DNA recovery among three sets of samples over three days. ( $\mathrm{n}=3 \pm$ standard error) 
Figure 56. Reproducibility of selectivity among three sets of samples over three days. Results were more reproducible for set E1S1 and E2S2 compared to E1S2. Selectivity was determined through calculation of peak height ratio of $\mathrm{Y}$ over $\mathrm{X}$ at the Amelogenin locus and male peak height over total peak height at the D871179 locus, $\mathrm{x}$ 100.( $\mathrm{n}=3 \pm$ standard error).

Figure 57. The effect of IMC pretreatment compared to original results with no treatment. Majority male profiles were obtained at every concentration with the addition of IMC treatment. Determined by male or female peak height divided by total peak height at 7 loci where no alleles are shared between the male or female contributor, $\mathrm{x} 100$. ( $\mathrm{n}=3 \pm$ standard error)

Figure 58. Profile of the female contributor. Panel represents loci labeled with 5-TAMRA (5-Carboxytetramethylrhodamine); Amelogenin, D8S1179, TPOX, and FGA.

Figure 59. Profile of the male contributor. Panel represents loci labeled with 5-TAMRA (5-Carboxytetramethylrhodamine); Amelogenin, D8S1179, TPOX, and FGA.

Figure 60. Sperm fraction profile of a 50:1 F:M sample extracted with the modified PCT + alkaline lysis protocol with IMC pretreatment. A percent male DNA contribution of $91 \%$ is observed in the profile. Panel represents loci labeled with 5-TAMRA (5-Carboxytetramethylrhodamine); Amelogenin, D8S1179, TPOX, and FGA.

Figure 61. Sperm fraction profile of a 100:1 F:M sample extracted with the modified PCT + alkaline lysis protocol with IMC pretreatment. A percent male DNA contribution of $86 \%$ is observed in the profile. Panel represents loci labeled with 5-TAMRA (5- Carboxytetramethylrhodamine); Amelogenin, D8S1179, TPOX, and FGA.

Figure 62. Figure 5. Sperm fraction profile of a 200:1 F:M sample extracted with the modified PCT + alkaline lysis protocol with IMC pretreatment. A percent male DNA contribution of $82 \%$ is observed in the profile. Panel represents loci labeled with 5-TAMRA (5- Carboxytetramethylrhodamine); Amelogenin, D8S1179, TPOX, and FGA 
Figure 63. Female DNA recovered in the captured fraction following IMC pretreatment. The greatest number of epithelial cells were predictably captured

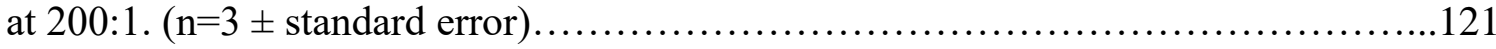

Figure 64. Stability of the method was tested with a 5-year-old post-coital sample. A male DNA contribution of 39\%was obtained. Determined by male or female peak height divided by total peak height at 7 loci where no alleles are shared between the

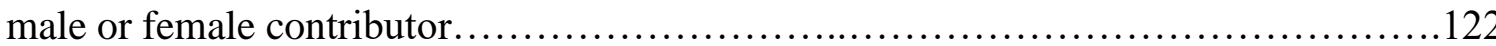

Figure 65. Female DNA recovered in the captured fraction and supernatant following IMC pretreatment of a 5-year-old post-coital sample. A concentration of $24.1 \mathrm{ng} / \mu \mathrm{L}$ was detected in the captured fraction with $43.2 \mathrm{ng} / \mu \mathrm{L}$ left behind in the

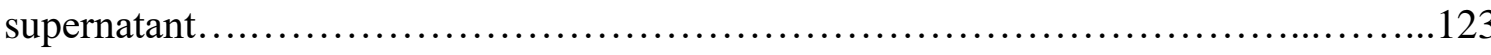




\section{ACRONYMS AND ABBREVIATIONS}

${ }^{\circ} \mathrm{C} \quad$ Degree Celsius

A Adenine

ABI Applied Biosystem by Life Technologies

ADE Acoustic Differential Extraction

AL-PEG Alkaline Lysis Polyethylene Glycol

ALS Alternative Light Source

AP Acid Phosphatase

bp Base Pair

C Cytosine

CCC-DNA Covalently Closed Circular DNA

CCD Charged Couple Device

CE Capillary Electrophoresis

CODIS Combined DNA Index System

Ct Cycle Threshold

DNA Deoxyribonucleic acid

dNTP Deoxyribonucleotide triphosphate

dsDNA Double Stranded DNA

DTT Dithiothreitol

EDTA Ethylenediaminetetraacetic Acid

EOF Electroosmotic Flow

FTA Fast Technology for Analysis of nucleic acids 


\begin{tabular}{|c|c|}
\hline $\mathrm{G}$ & Guanine \\
\hline GPG & Glass-PDMS-Glass \\
\hline $\mathrm{Hb}$ & Hemoglobin \\
\hline ILS & Internal Lane Standard \\
\hline IRB & Institutional Review Board \\
\hline IMBs & Immunomagnetic Beads \\
\hline IMC & Immunomagnetic Capture \\
\hline IPC & Internal Positive Control \\
\hline Iso-C & 5-methylisocytosine \\
\hline Iso-G & Isoguanine \\
\hline $\mathrm{LCM}$ & Laser-Capture Microdissection \\
\hline mAbs & Monoclonal Antibodies \\
\hline $\mathrm{MgCl}_{2}$ & Magnesium Chloride \\
\hline MOSPD3 & Motile Sperm Domain-Containing Protein 3 \\
\hline mRNA & Messenger Ribonucleic Acid \\
\hline PBS & Phosphate-Buffered Saline \\
\hline PBSO & Palm Beach County Sherriff's Office \\
\hline PCIA & Phenol- Chloroform Isoamyl Alcohol \\
\hline PCR & Polymerase Chain Reaction \\
\hline PCT & Pressure Cycling Technology \\
\hline PS & Phosphatidylserine \\
\hline PSA & Prostate Specific Antigen \\
\hline
\end{tabular}


PZT Piezoelectric Transducer

RFLP Restriction Fragment Length Polymorphism

RFU Relative Fluorescence Units

SDS Sodium Dodecyl Sulfate

SPAM1 Sperm Adhesion Molecule 1

ssDNA Single Stranded DNA

STRs $\quad$ Short Tandem Repeats

SWGDAM Scientific Working Group on DNA Analysis Methods

T Thymine

tACE Testicular Isoform of the Angiotensin-Converting Enzyme

TAPS 3-[[1,3-dihydroxy-2-(hydroxymethyl)propan-2-yl]amino]propane-1-sulfonic acid

Taq Thermus aquaticus Polymerase

TE Tris-EDTA

$\mathrm{T}_{\mathrm{m}} \quad$ Melting Temperature

VNTR Variable Number Tandem Repeats 


\section{CHAPTER I. DNA EVIDENCE}

\section{A. History of DNA Evidence}

Forensic techniques have formally existed since the 1900s with the introduction of the Henry Classification System for Fingerprint Analysis. Blood typing and microscopic analysis of ballistic evidence have also been used for the investigation of crime scene evidence [106]. But the history of modern forensic DNA techniques started in 1985 with Sir Alec Jeffreys of the University of Leicester and his discovery of variable number of tandem repeats (VNTRs). These are repeat sequences of ranging anywhere between 8-100 bp in length. The number of repeats vary between individuals making them useful as genetic markers for identification [52].

Sir Jeffreys used restriction fragment length polymorphism (RFLP) analysis to digest VNTR evidence into fragments with restriction enzymes. These fragments are separated by size through electrophoresis techniques, in which such fragments are migrate on a gel with an application of an electric field. The smaller fragments migrate faster and different bands are formed as the fragments progress. The resulting bands are detected through a technique called Southern Blotting. They are applied to a nylon membrane and probes with radioactive labels are attached. The bands are then visualized through X-ray film exposure. This technique came to be known as DNA fingerprinting $[52]$.

These genetic markers have fallen out of favor and short tandem repeats (STRs) have taken their place. These repeats are 2-7 bp in length making them useful for degraded evidence that may contain fragmented DNA [69]. Polymerase chain reaction (PCR) 
methods that allow for amplification of small amounts of DNA greatly increase the sensitivity of DNA analysis [72]. Together, these discoveries are the basis of modern forensic DNA analysis methods [105].

\section{B. DNA Biology}

\section{B.1. Introduction}

Cells are biological units referred to as the "building blocks of life". Within eukaryotic organisms, all cells, with the sole exception of red blood cells, contain a nucleus. Enclosed within this nucleus are deoxyribonucleic acid (DNA) molecules associated with histone proteins to form a complex called a chromosome. DNA contains genetic information for all forms of life [113]. The information found in DNA is transcribed into messenger ribonucleic acid (mRNA) that provides instructions for the synthesis of proteins from amino acid groups. [59]. 


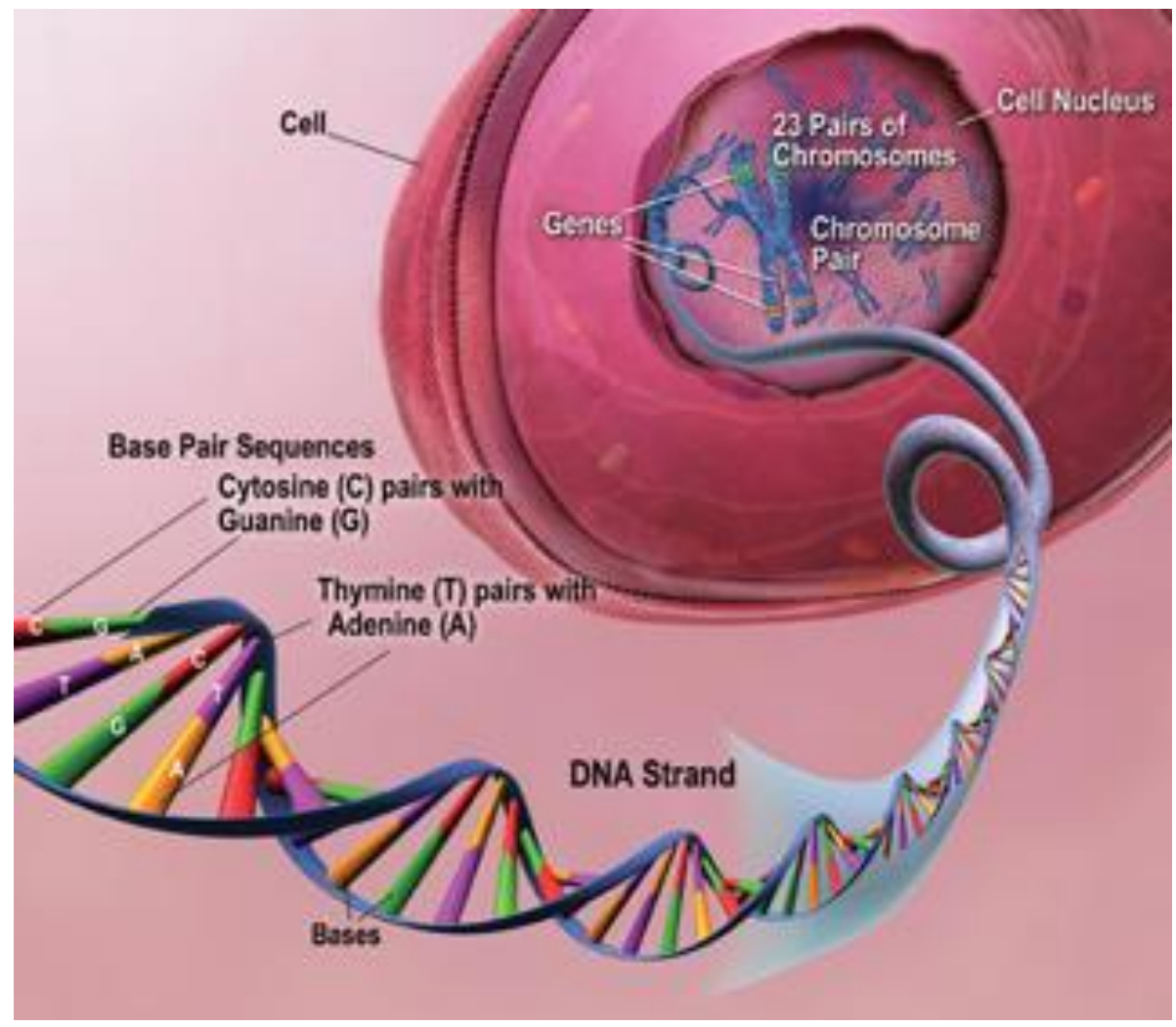

Figure 1. A DNA sequence is made up of 4 different nitrogenous bases; adenine, thymine, guanine and cytosine. Within the nucleus of each somatic cell, DNA is arranged into 23 pairs of chromosomes. Adapted from the National Institute of Health official website.

\section{B.2. DNA Structure}

Nucleic acid molecules are made up of deoxyribose sugars, phosphate groups, and nitrogenous bases. Together, these three molecules form a monomer or subunit of a nucleic acid called a nucleotide. Deoxyribose has a five-carbon ring structure. The phosphate groups form a phosphodiester bond between the 5 ' carbon of one sugar molecule and the 3' carbon on the next in the chain forming the "backbone" of the DNA molecule. [11].

The nitrogenous bases attach to the 1' carbon atom of the sugar molecule. There are four different bases; adenine $(A)$, guanine $(G)$, cytosine $(C)$, and thymine $(T)$. DNA 
exists as a double stranded molecule forming a double helix. These strands are connected through hydrogen bonds between bases forming complementary base pairs. Adenine is paired with thymine by two hydrogen bonds and cytosine is paired with guanine by three hydrogen bonds. The different sequences of these base pairs provide variation in the genetic code. One strand proceeds in the 5' to 3' direction while the other proceeds $3^{\prime}$ to 5' making the double helix antiparallel [11]. The double-stranded DNA (dsDNA) can be denatured into single-stranded DNA (ssDNA) with the application of high temperature or chemical methods. The three hydrogen bonds between cytosine and guanine make molecules with high cytosine content more difficult to denature [4].

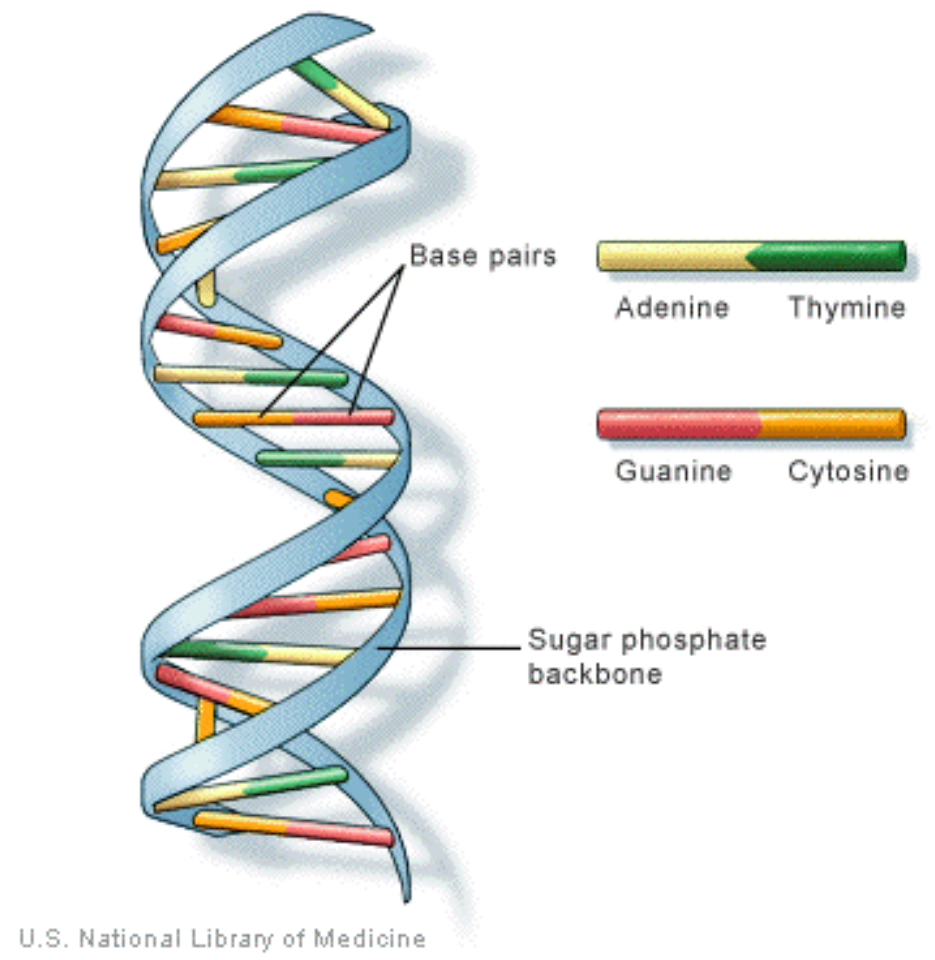

Figure 2. The DNA backbone is made up of phosphate groups that connect deoxyribose molecules. Nitrogenous bases bonded to the deoxyribose molecules are paired with complementary bases on the other strand in an antiparallel fashion forming a double helix. Adapted from the U.S. National Library of Medicine website. 


\section{B.3. Chromosomes}

DNA is associated with histone proteins complexes called chromosomes. Humans have 22 chromosome pairs called autosomes and an additional pair of sex chromosomes. These sex chromosomes are designated as $\mathrm{X}$ and $\mathrm{Y}$, with males having one of each and females having two X chromosomes. These can be used to identify the sex of an individual [113]. A complete set of chromosomes is referred to as a karyotype. Chromosomes contain subunits of DNA called genes which are made up of coding and non-coding regions referred to as exons and introns respectively. When DNA is transcribed into pre-mRNA, the intron sections are excised to form mRNA [59]. These introns are used for identification in forensics.

A section of a chromosome is known as a locus and the different forms of a gene at that locus are called alleles. When both chromosomes have the same allele, the individual is homozygous at that locus. When the alleles are different, the individual is heterozygous. A combination of different alleles at different loci are used for identification of an individual. The more loci included in a profile the higher the power of discrimination; the potential power to discriminate between any two individuals chosen at random [22].

Most cells are diploid and contain both sets of chromosomes. Half of an individual's chromosomes are inherited from each parent through the fusion of haploid gametes that only contain one set of chromosomes.

DNA is also found in mitochondria, organelles that convert molecules from food into energy. Unlike genomic DNA, mitochondrial DNA (mtDNA) exists as multiple 
copies in a cell and is passed down from the mother to children of either sex without recombination [59].

Freidrich Miescher is credited with the first identification of nucleic acids. This was followed by the discovery of nucleotide components by Phoebus Levine and the relationship between nitrogenous base pairs by Erwin Chargaff. These discoveries paved the way for further experiments involving X-ray crystallography by Rosalind Franklin and Maurice Wilkins that elucidated the structure of the DNA molecule. Their work then led to the discovery of the double helical structure of DNA by James Watson and Francis Crick [89]. Current research continues to enhance our understanding of the nature of DNA and the human genome. New technologies that allow for rapid sequencing of large amounts of DNA will undoubtedly lead to exponential advances in our knowledge of DNA and molecular biology. 

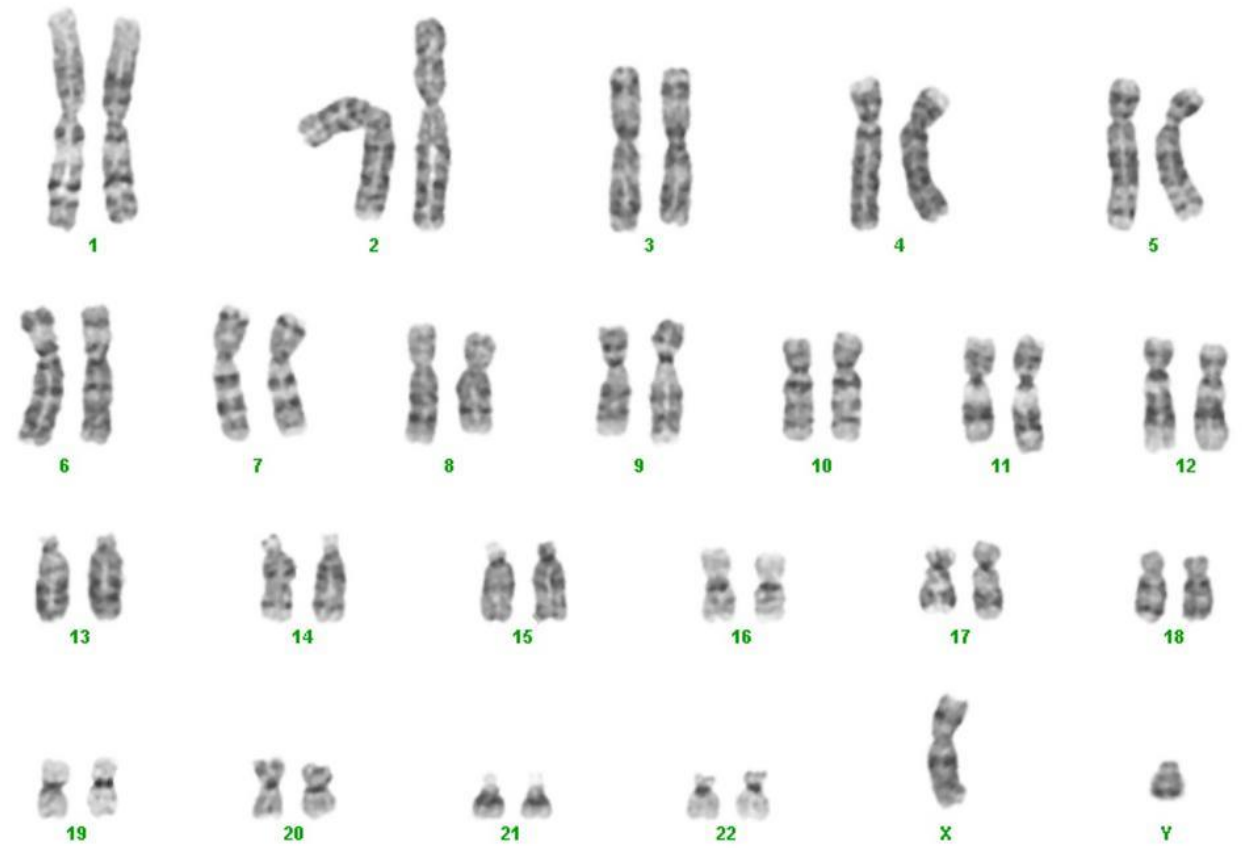

Figure 3. A healthy human male has a karyotype of 22 pairs of autosomes and an $X$ and $Y$ chromosomes. Adapted from Broad Institute website.

\section{Biological Evidence}

\section{C.1. Introduction}

Biological evidence must be carefully documented and collected at a crime scene by crime scene investigators. This evidence includes stains and samples plainly visible to the naked eye, but also stains revealed through screening methods and other types of samples that may contain trace DNA. Reference samples collected from victims and family members, commonly in the form of buccal swabs, are very important evidence for exclusion purposes [106]. Negative controls should also be taken from substrates that have been left unstained by evidence [22]. 
Samples are air-dried in order to prevent bacterial growth that can lead to degradation of DNA evidence and are transported in paper envelopes rather than plastic bags to prevent decomposition. Storage frequently takes place at $4^{\circ} \mathrm{C}$ or $-20^{\circ} \mathrm{C}$ for better preservation [83]. Simple presumptive tests to exclude or include the presence of a type of bodily fluid are often performed. This is then followed by more expensive confirmatory tests [9].

\section{C.2. Bloodstain Evidence}

Bloodstains are one type of evidence that may frequently be recovered from the scene of a violent crime. The splatter pattern of a stain can reveal information about the event and how the stain was formed [58]. While DNA evidence from a bloodstain is obtained from white blood cells, hemoglobin $(\mathrm{Hb})$ found in red blood cells is essential for screening, presumptive, and confirmatory tests that are performed to determine the presence of blood at a crime scene [10].

Screening with luminol (5-amino-2,3-dihydro-1,4-phthalazinedione) $\left(\mathrm{C}_{8} \mathrm{H}_{7} \mathrm{~N}_{3} \mathrm{O}_{2}\right)$ is a common practice. With the addition of luminol and a hydrogen peroxide solution, hemoglobin undergoes a chemiluminescence reaction. This allows for the visualization of trace amounts of blood. DNA and other types of evidence are not damaged by this process and remain intact for further analysis, but the blood splatter pattern may be disrupted. The presence of metals, bleach, or vegetable peroxidases can result in false positives which prevents this test from being considered confirmatory [10].

The Kastle-Myer test is a presumptive test for blood that also utilizes the oxidant properties of hemoglobin molecules. Phenolphthalin is a colorless compound that is 
oxidized in the presence of blood and hydrogen peroxide. It is converted into Phenolphthalein which has a pink color. This test can produce false positives if exposed to other oxidants [35].

The ABAcard ${ }^{\circledR}$ HemaTrace ${ }^{\circledR}$ for the Forensic Identification of Human Blood (Abacus Diagnostics Inc., West Hills, CA) can confirm the presence of blood through antibody-antigen reactions. The test strips contain immobilized polyclonal antihuman antibodies. The analyte is treated with monoclonal antihuman hemoglobin antibodies with an incorporated pink dye. When the hemoglobin in the analyte binds to the immobilized antibodies in the test region, the visible pink line confirms the presence of blood. The test strip includes a control region further downstream where excess antibodies are bound. The formation of a pink line confirms that the test functioned as expected and any negative result was due to lack of blood in the sample and not to any failure on the part of the reagents or analyst [103].

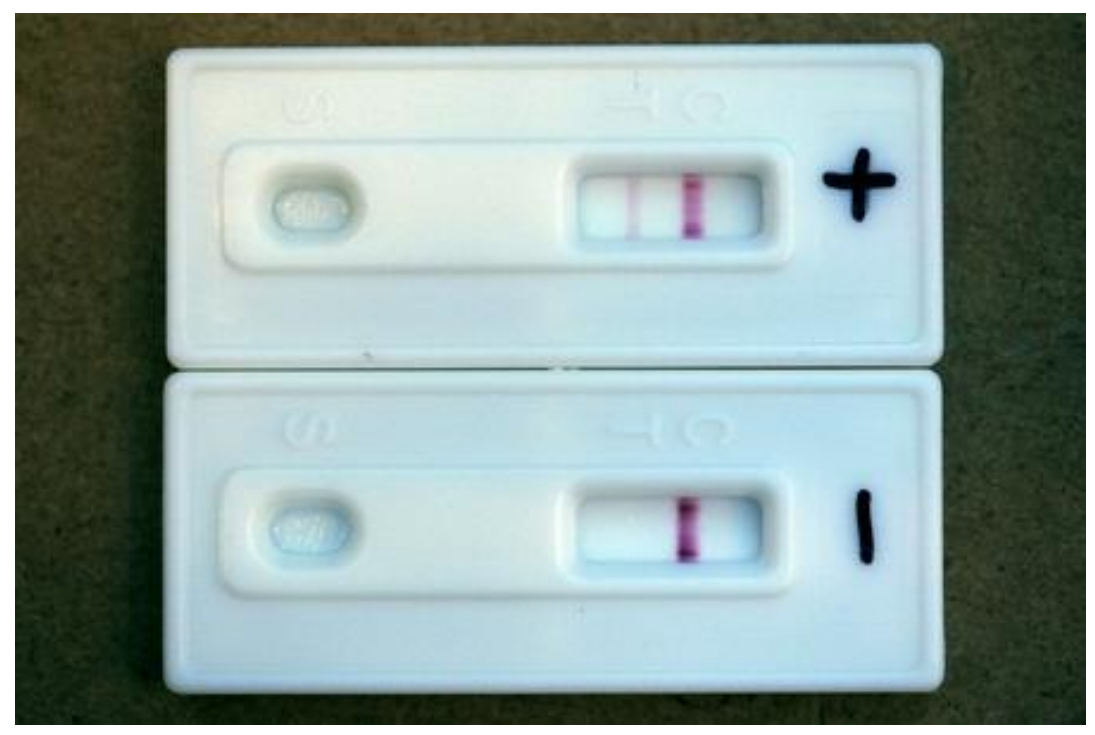

Figure 4. ABAcard ${ }^{\circledR}$ HemaTrace $®$ for the Forensic Identification of Human Blood. Above is a positive result indicated with a pink line at the test region on the left as well as a positive 
result at the control region. Below is a negative result with a pink line at the control region only. Adapted from the Minnesota Bureau of Criminal Apprehension website.

Dried blood may be collected through scraping with a sterilized blade while smaller stains may be collected with a swab. Double-swabbing is often used to collect sample with a moist swab followed by a dry swab to collect any leftover evidence. Larger wet stains are recovered with a sterile absorbent while clothing is cut and stored in paper envelopes [58].

\section{C.3. Seminal Evidence}

The examination of sexual assault evidence often involves tests for the presence of semen. Alternate light sources (ALS) can be used to visualize semen stains through the presence of flavin molecules that have a tendency to fluoresce when exposed to $450 \mathrm{~nm}$ wavelengths [67]. The detected area can then be outlined for later evidence collection and analysis [61].

Acid phosphatase (AP) is produced by the prostate gland and is present in semen in greater amounts than other bodily fluids. It can be detected through the application of sodium alpha naphthyl phosphate and diazo blue dye solution. AP reacts with these reagents and produces a purple color. This enzyme is found in smaller concentrations in other bodily fluids including vaginal secretions, feces, and occasionally in blood [109].

Confirmatory tests include microscopic visualization of sperm cells. This can be simplified by the use of a Christmas tree stain. Nuclear fast red stains nucleic acids in the sperm head red and picroindigocarmine stains the sperm tails green. Epithelial cells commonly found in sexual assault evidence can also have nuclei and cell membranes 
stained in this fashion, but the cells are easily distinguished from one another [67]. This test may be complicated by the existence of oligospermic, azoospermic, aspermic, or vasectomized individuals that produce either no mature sperm cells or very few [22].

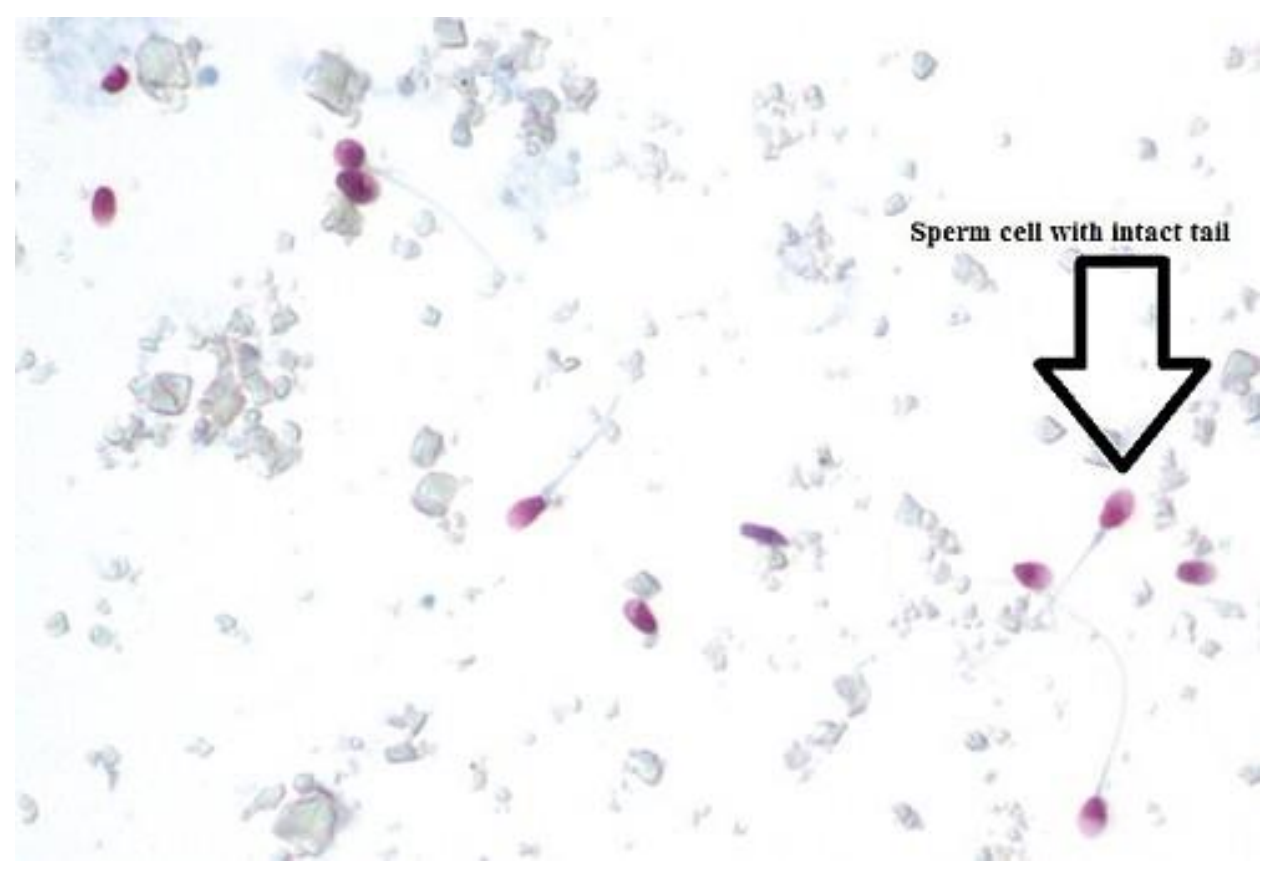

Figure 5. A Christmas tree stain. Nucleic acids in sperm cell heads are stained red and tails are stained green for improved visibility. Adapted from St. Louis County Police Crime Laboratory website.

Another confirmatory test ABAcard ${ }^{\circledR}$ p30 for the Forensic Identification of Semen (Abacus diagnostics Inc., West Hills, CA) targets the prostate specific antigen (PSA) p30 that is only present in semen and functions in a similar manner to the HemaTrace ${ }^{\circledR}$ test. Unlike microscopic examination methods, it does not require the presence of sperm cells to be effective [44].

\section{C.4. Epithelial Cells and Touch DNA}

Epithelial cells appear as problematic components in sexual assault evidence that must be separated from the sperm cells present in the sample for a clear suspect profile 
[33]. They may also be sought after as evidence in violent crimes under the victim's fingernails in hopes that they have been deposited after scratching during a struggle. The fingernails are clipped and collected on a cloth. The nail clippings, clippers, and cloth are all packaged and transported in a paper envelope. The collected evidence may undergo microscopic examination to confirm the presence of epithelial cells. The cells can be collected for extraction through swabbing, scraping, or the entire nail may be placed in extraction buffer. Despite these procedures, epithelial cells belonging to the suspect are rarely found in this way or are overwhelmed by the victim's cells in a sample [43].

Epithelial cell evidence may also be deposited as touch samples. Shed skin cells can be left behind when a suspect touches an object or person [2]. Touch DNA has been recovered from many different types of objects and surfaces such as keyboards, paper, bedding, fabric, pens, doorknobs, firearms, and briefcase handles. Samples may be collected through tape lifting or swabbing [40]. As with other types of evidence, a doubleswab technique is recommended for better recovery [83]. There is some doubt as to whether these epithelial cells actually come from "touch" or whether they may have been transferred through other methods such as saliva. Concerns have been expressed about such evidence being given undue weight as the true source may be uncertain [40] and the reliability of such low-template DNA has come into question as well [56].

\section{C.5. Saliva Stain Evidence}

Buccal epithelial cells can be recovered from saliva stains. This evidence is commonly encountered on drinking glasses, bottles, cigarette butts, chewing gum, and 
even on bite marks [1]. Like semen, it may be visualized through alternate light sources [67].

Saliva contains an enzyme called amylase which breaks down starches. The presumptive starch-iodine test detects the presence of saliva through interaction with amylase. Iodine particles become trapped in starch polymers leading to the production of a purple starch-iodine complex. Amylase can hydrolyze the starch molecules which frees the iodine and the purple color disappears [53].

Phadebas $^{\circledR}$ (Magle Life Sciences, Cambridge, MA) tablets operate on a similar principle, but use a blue dye cross-linked to starch polymers. The presence of amylase breaks down the starch and releases the blue dye into the solution [108].

Amylase is detectable in saliva stains for a long as 28 months, making it remarkably stable. Unfortunately, it can be found at lower levels in other bodily fluids such as semen and vaginal secretions. Albumin and gamma-globulin in blood and semen can also react with starch leading to false positives [51].

\section{C.6. Hair, Teeth, and Bone Evidence}

Nuclear DNA can be recovered from the keratinocyte skin cells present around the root bulb [69]. Naturally shed telogen hairs or hair shafts do not contain much nuclear DNA, but multiple copies of mtDNA may be present [48]. Hair evidence can be collected with lifting tape or sterilized forceps. Lifting tape can be particularly useful when hair is not visible at the scene. Vacuums can be used to collect both hair and fibers, but 
contamination on the vacuum filter where the evidence is deposited can be a problem [93].

Bite mark analysis can be used to identify suspects through specific dental impressions [7]. The examination of skeletal remains can give clues to a victim's identity through comparison to dental or medical x-rays or through signs of age, sex, or trauma $[79,20]$.

The power of discrimination and sensitivity provided by modern DNA methods and technology allow for wider range of evidence collection at a crime scene. It also increases the problem of sample contamination. In order to maintain the integrity of collected evidence, care must be taken by crime scene investigators, forensic scientists in proper handling and storage of evidence and maintenance of the chain of custody.

\section{CHAPTER II: DNA EXTRACTION}

\section{A. Extraction Methods}

\section{A.1. Introduction}

The goal of extraction methods is the recovery of DNA from collected biological evidence. These methods involve the separation of cells and genetic material from the substrate as well as the lysis of cell membranes to release DNA and other components. This is followed by purification of the DNA from any contaminants and unwanted material [30]. These methods have different advantages and disadvantages that may depend on the type of evidence under investigation. 


\section{A.2. Organic Extraction}

Organic extraction requires the use of a detergent such as sodium dodecyl sulfate (SDS) to facilitate cell lysis through disruption of the cell membrane, Proteinase $\mathrm{K}$ for the hydrolysis of histone proteins, and, in some cases, Dithiothreitol (DTT) for the reduction of disulfide bonds found in sperm cells. Incubation in a water bath set to $56^{\circ} \mathrm{C}$ normally follows which allows for more effective cell lysis and deactivates Proteinase K [33].

Organic extractions are commonly purified with phenol-chloroform isoamyl alcohol (PCIA) (25:24:1 v/v). Isoamyl alcohol acts as an anti-foaming agent while phenol-chloroform is added in equal volume to the sample to allow for polar DNA molecules to partition into the aqueous phase while unwanted contaminants, such as proteins and lipids, remain in the organic phenol-chloroform layer [65]. Ethanol precipitation allows for the collection of a DNA pellet which is then subjected to washing steps prior to dissolution in nuclease-free water or Tris-EDTA (TE) buffer for a final desired concentration for analysis [28]. The multiple handling steps and washes are time consuming and can lead to sample loss or contamination. Phenol itself can behave as an inhibitor [68] and is also a suspected carcinogen [97] making its use undesirable if safer and more efficient methods are at hand.

Extraction with proteinase alone is possible with application of proteolytic enzymes obtained from thermophilic species Bacillus EA1 [71]. The procedure is a simple, closed-tube extraction method with few handling steps which limits the chances for contamination [63]. It begins with incubation at $75^{\circ} \mathrm{C}$ for 15 minutes. This is followed 
by incubation at $96^{\circ} \mathrm{C}$ for 15 minutes which deactivates the enzyme and further promotes cell lysis. It has been successfully applied to swabs, drinking glasses, gloves and socks. But the presence of inhibitors in black denim and cigarette butts have been shown to decrease effectiveness of the technique [71].

\section{A.3. Chelex ${ }^{\circledR} 100$ Extraction}

Chelex (Bio-Rad Laboratories, Hercules, CA) is a cation-exchange resin consisting of styrene divinylbenzene copolymer with paired iminodiacetic ions. These

ions bind divalent co-factors such as $\mathrm{Mg}^{2+}$ that are necessary for the activation of nucleases and inhibitors such as porphyrin that are released from heme groups in blood stain samples [45]. The advantages of this extraction method includes its simplicity which involves boiling the sample in a solution of 5\% Chelex 100 solution and deionized water. Although it binds nuclease co-factors and certain inhibitors, Chelex resin itself can lead to inhibition if left behind in the sample and high temperatures can further damage degraded DNA [114, 45].

\section{A.4. FTA ${ }^{\text {TM }}$ Paper}

Fast technology for Analysis of nucleic acids (FTA) serves as a collection, storage, and extraction method for DNA. The paper contains reagents such as weak acids, surfactants, chelating agents, and uric acid that protect the DNA entangled in the matrix from nucleases, microbes, and other environmental sources of contamination and degradation [104]. FTA ${ }^{\mathrm{TM}}$ paper is compact for easy storage. Samples remain stable and can be maintained long term at room temperature. There is no need for quantitation as each disk has a predictable amount of DNA according to size [101]. FTA ${ }^{\mathrm{TM}}$ paper disks 
can be added directly to PCR reactions for amplification after multiple washings with Tris-EDTA (TE) buffer (pH 8.0) and drying at room temperature or with gentle heat [35].

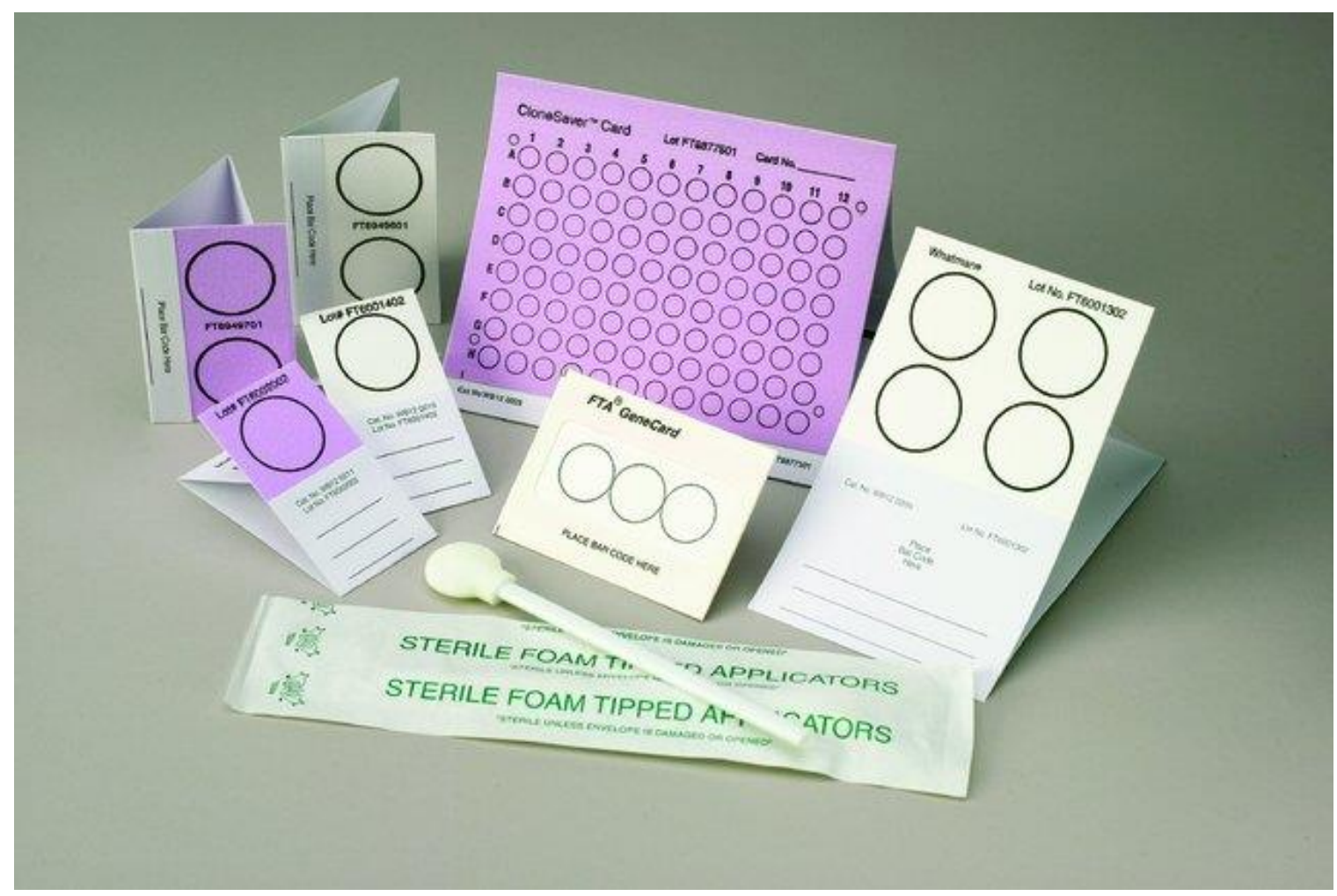

Figure 6. Whatman® FTA® card technology from Sigma-Aldrich (St. Louis, MO). Adapted from Sigma-Aldrich website.

\section{A.5. Solid-phase Extraction}

Solid-Phase extraction is a procedure employed for the simplification of the DNA purification step. In this method DNA is bound to a solid phase, often silica, while unwanted material remains unbound and is washed away [38]. QIAamp ${ }^{\circledR}$ (Qiagen, Inc., Valencia, CA) kits utilize chaotropic salts in acidic conditions. The salt disrupts the shell of hydration around biomolecules and forms a salt bridge between DNA and silica beads. The DNA is washed first with water to remove impurities, then with ethanol for removal 
of salt, then again with water again to remove any residual ethanol. The $\mathrm{pH}$ is increased and salt concentration is lowered allowing the DNA to be released and eluted [73].

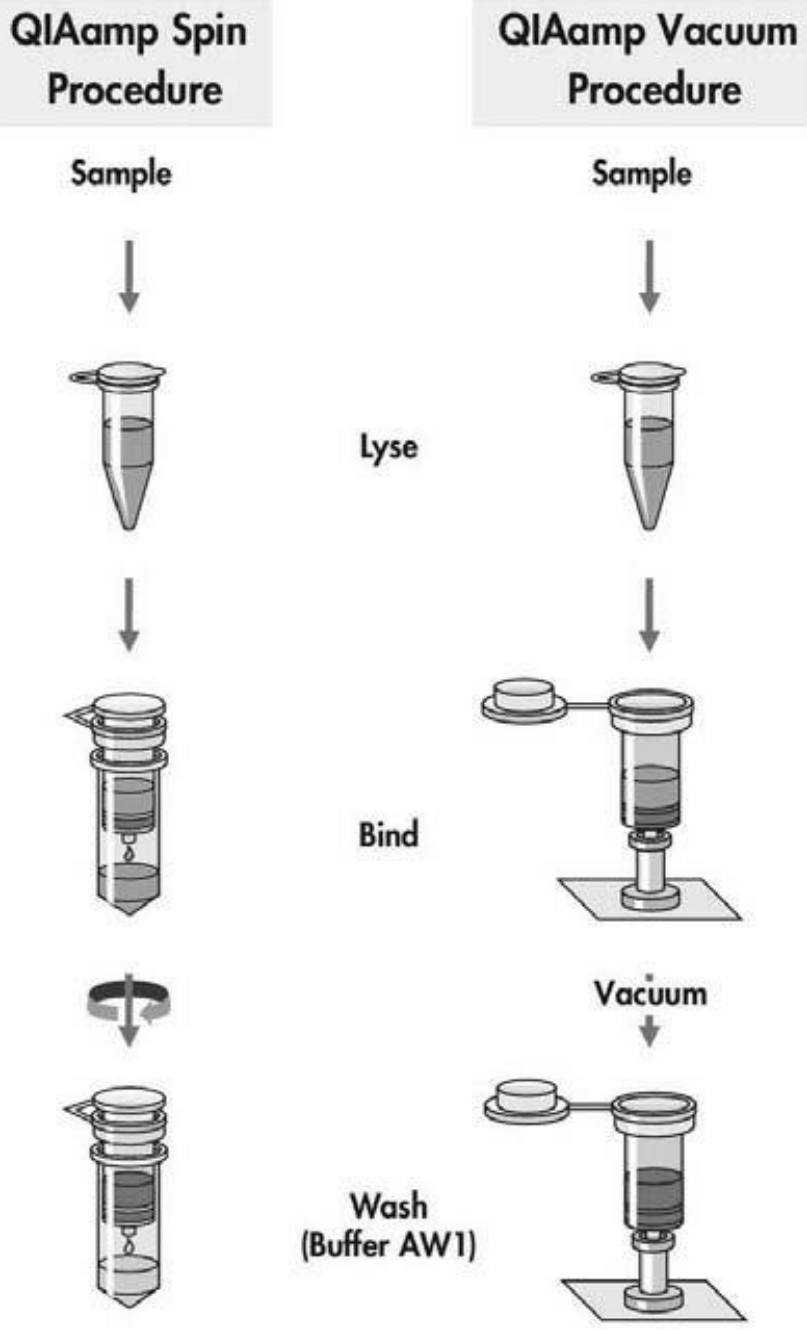




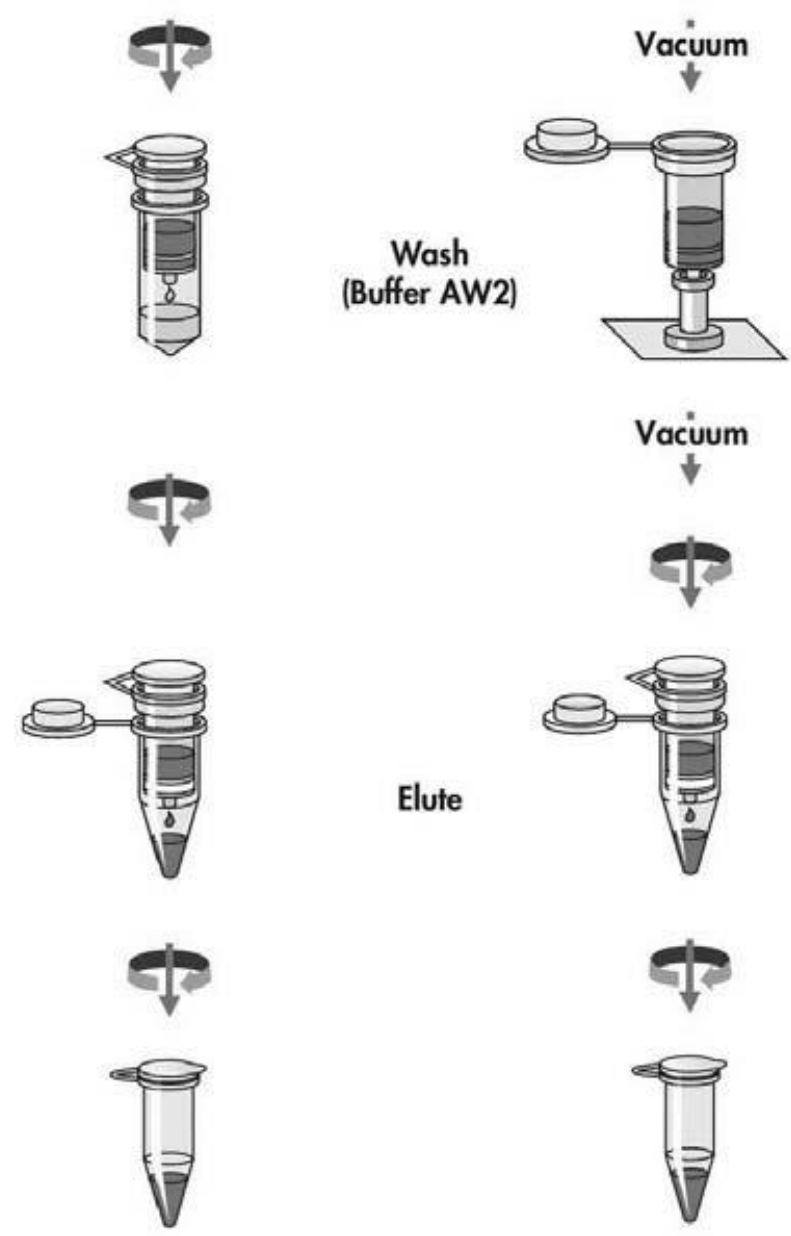

Pure genomic or viral DNA

Figure 7. A general QIAamp procedure. Centrifugation (spin) or vacuum manifold may be employed. In either case the process involves, lysis, binding of DNA, washing, and elution. Adapted from Qiagen website.

The DNA IQ ${ }^{\mathrm{TM}}$ System (Promega Corporation, Madison, WI) uses silica-coated paramagnetic resin that binds DNA with chaotropic salt and immobilizes it with a magnet. This allows for multiple washings to proceed without disturbance of the bound DNA. The DNA is then released into elution buffer with incubation at $65^{\circ} \mathrm{C}$ for 5 minutes [31]. Impurities that can interfere with the functioning of the magnet may lead to loss of sample [86]. 
Automated versions of this process utilizing $\mathrm{pH}$ changes and magnetic beads are included in instruments such as BioRobot EZ1 (Qiagen, Inc., Valencia, CA) and QIAcube (Qiagen, Inc.,Valencia, CA) allowing for greater convenience and efficiency [17].

\section{A.6. ChargeSwitch ${ }^{\circledR}$ Technology}

ChargeSwitch $^{\circledR}\left(\mathrm{CST}^{\circledR}\right.$, Life Technologies, Grand Island, New York) magnetic beads are made of an ionizable nucleic-acid binding ligand. At $\mathrm{pH}$ levels of below 6.0, the DNA binds to the positively-charged beads. Magnetic immobilization allows for the removal of liquid solution and washing away of impurities. The magnet is removed and the beads are washed at $\mathrm{pH}$ levels of 7.0. The purified DNA is then eluted at a $\mathrm{pH}$ of over 8.5. This neutralizes the surface charge of the beads and releases the DNA [115].

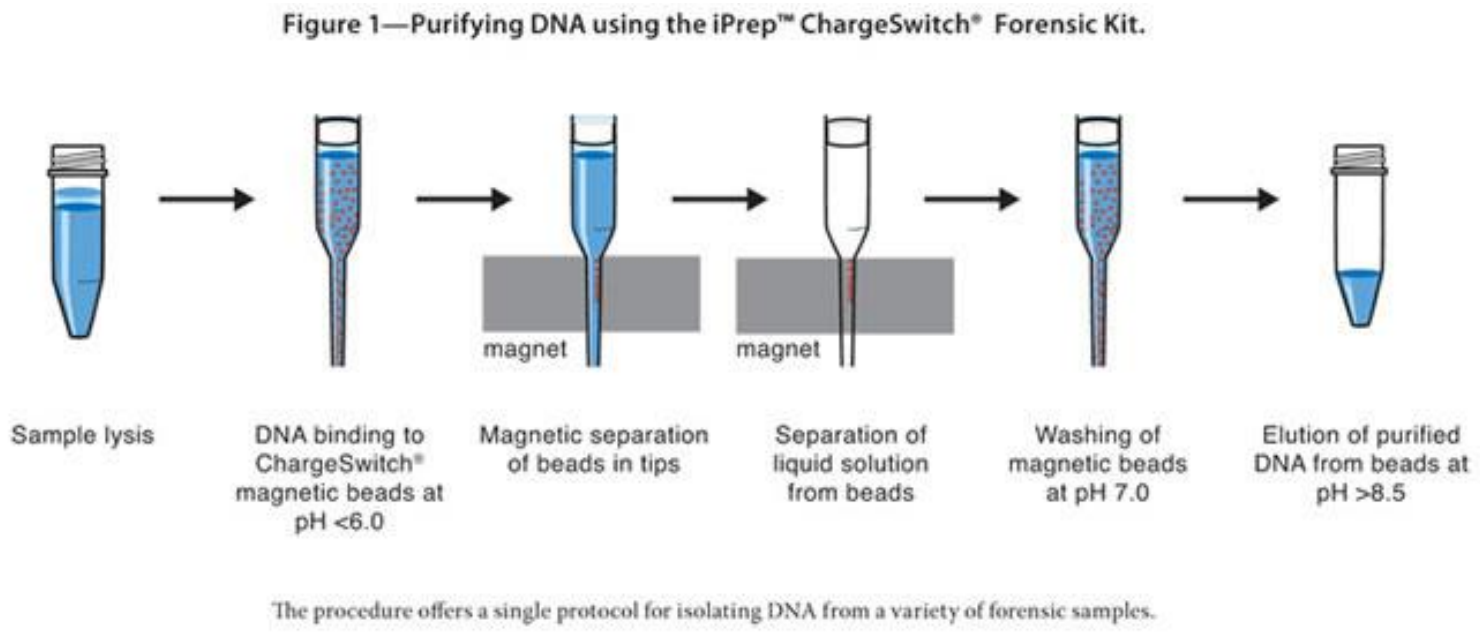

Figure 8. ChargeSwitch ${ }^{\circledR}$ purification. After sample lysis, the pH is altered to less than 6.0 for DNA binding to magnetic beads. The DNA bound to the beads is immobilized with a magnet and impurities are washed away. The pH is increased to 7.0 and the magnetic beads are washed. Increasing the $\mathrm{pH}$ to more than 8.5 releases the purified DNA. Adapted from ThermoFisher website. 


\section{A.7. Alkaline Lysis}

The alkaline lysis method of extraction was originally developed to purify plasmid DNA from bacterial cells [14]. Alkaline conditions denature and solubilize proteins through ionization of amino acid residues. This ionization leads to the disruption of both cellular and nuclear membranes as well as the dissolution of DNA molecules [54]. In a narrow $\mathrm{pH}$ range, 12.0-12.5, covalently closed circular DNA (CCC-DNA) is not disrupted which allows for the lysis and removal of linear DNA and high molecular weight RNA present in a sample with the inclusion of lysozymes to weaken the cell wall, detergents such as SDS, and alkaline solutions. The addition of a neutralization agent such as sodium acetate and ethanol results in precipitation of contaminants which are then pelleted leaving CCC-DNA behind in the supernatant [14].

Modifications to this protocol have been used recently for the detection of Salmonella. The Alkaline lysis polyethylene glycol (AL-PEG) method was applied to colonies dissolved in distilled water. Lysis was achieved with the addition of $500 \mu \mathrm{l}$ of AL-PEG reagent $(60 \mathrm{~g}$ PEG $200+930 \mu \mathrm{KOH}+39 \mathrm{~mL}$ water $)$ and incubation at $60^{\circ} \mathrm{C}$ for 10 minutes. Results were comparable to those attained with commercial kits with the added benefit of a shorter processing time [90].

Plasmid purification through alkaline lysis has also been performed to purify amplified mtDNA fragments directly cloned into a vector for both forensic and anthropological applications. Specifically, the method was successfully applied for the examination of mtDNA evidence in a rape case and to check for contamination in ancient skeletal remains [42]. 
Forensic evidence consisting of blood stains, semen stains, and buccal swabs have been extracted with alkaline lysis methods. Rudbeck et al. utilized $20 \mu 1$ of $0.2 \mathrm{~N}$ $\mathrm{NaOH}$ to samples with incubation at either room temperature at $75^{\circ} \mathrm{C}$ for only $5-10 \mathrm{~min}$ depending on the type of sample being extracted [91]. In every case, alkaline lysis has been described as a rapid, simple, and inexpensive technique for single source samples with the added benefit of deactivating nucleases and diluting inhibitors [54].

There are many different extraction methods available to forensic scientists. In all cases the goal is the same, removal of DNA from the substrate, cell lysis, and purification to maximize yield of pure DNA and obtain a full profile. The selected methods depend on many factors including the type of evidence, presence of inhibitors, and expense of the procedure.

\section{B. Differential Extraction}

\section{B.1. Introduction}

Processing of sexual assault evidence often requires the separation of the victim's epithelial cells from sperm cells in the substrate. Most methods devised for this purpose take advantage of the different structures of these cells. The epithelial cells are usually preferentially lysed and removed while leaving behind sperm cells that remain relatively undisturbed due to the presence of disulfide bonds that require additional reagents to disrupt [22]. Other methods involve the use of microfluidic devices that separate the cells based on their disparate sizes or lasers to directly capture sperm cells $[36,16]$. 


\section{B.2. Organic Differential Extraction}

Conventional differential extraction involves an initial step for the lysis of female epithelial cells in the sample with extraction buffer and Proteinase K [33]. After incubation and centrifugation, the supernatant containing the female DNA is removed. The sperm pellet left behind is washed to remove residual female DNA and lysed with the addition of Dithiothreitol (DTT) to reduce the disulfide bonds in the sperm cell that make it resistant to organic extraction. This process can be time-consuming, laborious, difficult to automate, and often results in poor recovery of DNA from the swab used to collect the sample [111]. The female DNA or undigested epithelial cells may also be left behind in the sperm cell pellet leading to a mixed genotype. The necessary number of washings and centrifugations during this process is determined by the examiner. This makes results dependent on user expertise.

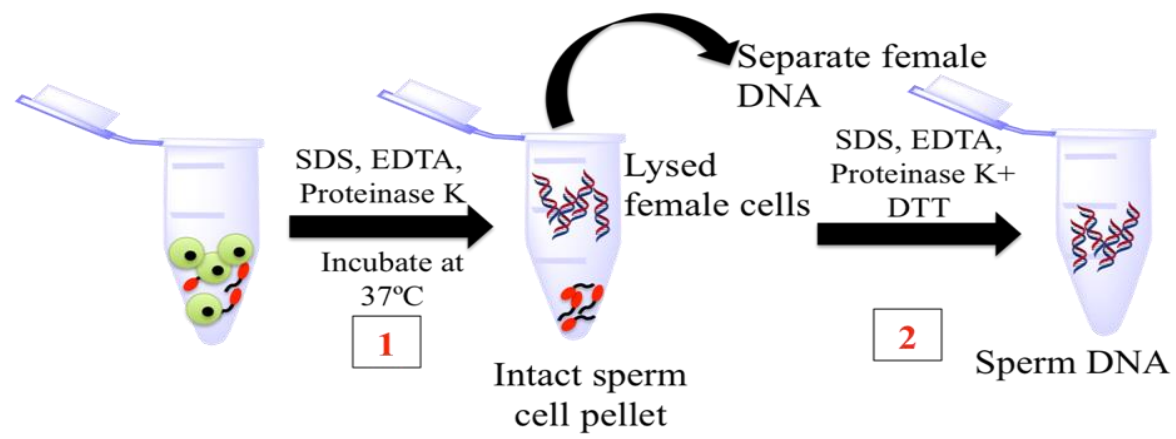

Figure 9. General organic differential extraction procedure. The first step involves incubation with the addition of detergent, buffer, and Proteinase $K$ for epithelial cell lysis. After centrifugation, the supernatant is removed and the intact sperm pellet is left behind. Several wash steps often follow. Incubation with the addition of detergent, buffer, and DTT lyses the sperm cell releasing the DNA for subsequent purification and analysis. 


\section{B.3. Methods for Increased Recovery}

Chemical methods have been devised to improve recovery of sperm cells from cotton swabs. The application of Sarkosyl and SDS were determined to result in sperm cell recoveries of $54.4 \pm 1.87 \%$ and $78.5 \pm 0.7 \%$ respectively. Both of these were significant improvements compared to the $39.4 \pm 2.1 \%$ recovered with differential extraction buffer $(0.01 \mathrm{M}$ Tris $-\mathrm{HCl} \mathrm{pH}$ 8.0, $0.01 \mathrm{M}$ diaminoethanetetraacetic acid (EDTA), $0.1 \mathrm{M} \mathrm{NaCl}, 2 \%(\mathrm{w} / \mathrm{v}) \mathrm{SDS}$ ). (Biorad, Hercules, CA). Storage time had an effect on results with longer times requiring more detergent for elution [76].

Enzymatic digestion was another method investigated for improving DNA recovery. Cellulase, enzymes that catalyze the decomposition of cellulose, was applied to break down cotton fibers and promote the release of cells from the swab. Sample swabs treated with cellulose from Aspergillus niger, Trichoderma reesi, and Trichoderma viride were compared to results from swabs treated with differential extraction buffer alone. All cellulase treated swabs displayed greater sperm cell recoveries with $T$. viride showing the best results [110]. Such treatments were attempted with cotton swabs and pressure-based extraction, but reported no improvement with any variety of cellulase at any concentration [75].

Nylon flocked swabs (MicroRheologics, Brescia, Italy) are claimed by the manufacturer to exhibit both improved sample absorption and sample release compared to typical cotton swabs. In order to test these claims, cotton swabs and nylon flocked swabs were tested with dried saliva stains and three different Qiagen extraction methods; QIAcube, BioRobot EZ1, QIAamp DNA Investigator Kit (Qiagen, Inc., Valencia, CA). 
Surprisingly, the highest recovery was reported with cotton swabs extracted with QIAamp and the lowest with nylon flocked swabs processed with BioRobot EZ1. The authors noted that previous tests with nylon flocked swabs were conducted with abundant amounts of moist sample rather than with dried samples as in this study. These findings support the conclusion that different swab types may be best suited for different types of samples [17].

\section{B.4. Differex ${ }^{\mathrm{TM}}$ System}

Promega produces a differential extraction kit, the Differex ${ }^{\mathrm{TM}}$ System (Promega Corporation, Madison, WI). The epithelial cells in the Differex ${ }^{\mathrm{TM}}$ protocol are still digested with the use of Proteinase K. After centrifugation, resin is added to the sample and the tube is inserted into a magnet. This allows the resin to cap and protect the resulting sperm pellet. The epithelial cell lysate can then be removed and the pellet is washed several times. A separation solution is used to protect the pellet from any solubilized DNA in the washing solution. The sperm pellet is then treated with a lysing solution containing DTT. This method can be automated and leads to a reduction in variability according to the manufacturer, but it still requires many washings and centrifugations to obtain a clean sample. Proteinase K digestion is still required for epithelial cell lysis which adds at least 90 minutes to the total extraction time. At least 2 hours are needed before the samples are ready for purification [107].

\section{B.5. Erase Sperm Isolation Kit}

The Erase Sperm Isolation Kit (Paternity Testing Corporation, Colombia, MO) applies selective degradation with nuclease for purification of the sperm pellet. A master 
mix of extraction buffer and Proteinase $\mathrm{K}$ is prepared and added to a solid substrate in a $2 \mathrm{~mL}$ Dolphin tube, designed for pelleting samples, for incubation at $56^{\circ} \mathrm{C}$ for 1 hour. The sperm cells are then pelleted through centrifugation. The substrate is discarded and the supernatant is transferred to a new tube for a saved epithelial fraction. A solution containing nuclease is added to the sperm pellet and it is incubated at $37^{\circ} \mathrm{C}$ for 15 minutes to digest the epithelial cell DNA left behind. A third solution is added and the sample is incubated at $56^{\circ} \mathrm{C}$ for another 15 minutes to deactivate the nuclease and lyse the sperm cells. Several wash steps can be included to the procedure as determined by the user [27].

\section{B.6. QIAcube}

Automated systems have also been devised for differential extraction such as the QIAcube (Qiagen, Inc., Valencia, CA). The process begins with epithelial cell lysis with extraction buffer and Proteinase $\mathrm{K}$ at $56^{\circ} \mathrm{C}$ for 2 hours. The resulting mixture is then loaded into the instrument where the sperm cells are pelleted and the lysate is removed. Four washing steps proceed with Buffer G2. This is then followed by digestion with sperm lysis buffer. These combined automated steps are complete after 70 minutes. Evaluation of this procedure determined that processing time was not decreased, but automation may lead to a reduction in human error [26].

\section{B.7. Laser Capture Microdissection}

Laser Capture Microdissection (LCM) has been suggested for the purpose of directly recovering sperm cell DNA from a sample. The sample is transferred to microscope slide and stained. Sperm cells are identified and a laser is used to excise and separate them into a collection tube for extraction. Studies have shown that a minimum 
of 30 sperm cells are recommended to achieve a full profile while the diploid epithelial cells require only 15 . This technique can certainly yield the desired results, but time and expense remain a problem [16].

\section{B.8. Microfluidic Devices}

Microfluidic devices are described as "self-contained closed systems for analysis" that will allow for automation and potentially decrease sample loss or contamination of evidence. Horsman et al. attempted cell separations with a microfabricated device that took advantage of differences in size and surface area between epithelial and sperm cells, which have diameters of 40-60 $\mu \mathrm{m}$ and 4-6 $\mu \mathrm{m}$ respectively. The cells were introduced into the microchip and allowed to pass through at a low flow rate. The epithelial cells, because of their greater size, would pass through more slowly and deposit themselves at the bottom of the inlet reservoir where they would undergo adsorption on the glass surface due to their relatively high surface area. This tendency would be increased by the occurrence of cell aggregation. The smaller sperm cells pass through to the outlet reservoir where they are captured separately from the epithelial cells. This technique was not tested with older samples. And free DNA from lysed epithelial cells could easily pass to the outlet reservoir along with the sperm cells leading to mixtures [47].

Different methods have been used to increase the efficiency of such devices. Some have circumvented the problem of lysed epithelial cells present in the sample by lysing epithelial cells prior to injection and depending on the size differences between intact sperm cells and free DNA for separation. Acoustic differential extraction (ADE) of 
sperm cells has been attempted with standing acoustic waves generating a force that acts on and traps objects such as intact sperm cells allowing them to be retained in a chamber of the device. Epithelial cell DNA passes through and is separated from the sperm rich fraction to be collected in a "female" outlet. Once the standing wave is terminated, the sperm cells are allowed to flow into a "male" outlet for collection [77].

Work continued with this technique with the inclusion of a glass-PDMS-glass (GPG) resonator chamber and external piezoelectric transducer (PZT). This increased the flow rate and throughput of the process to $30 \mu \mathrm{L} / \mathrm{min}$ which was previously limited to 1 $\mu \mathrm{L} / \mathrm{min}$. Polymeric beads were also added to assist in the trapping of sperm cells and further increase efficiency. Sample concentrations required for successful separation were previously as high as 500 cells $/ \mu \mathrm{L}$ for a $1 \mathrm{~mL}$ sample. The modifications allowed for processing of 1 sperm cell/ $\mu \mathrm{L}$ in a $1 \mathrm{~mL}$ volume [116].

Modifications to microfluidic devices such as these can result in more complicated microfabrication processes, particularly when external additions such as transducers are included in the design [62]. Simpler designs have been tested that separate cells through hydrodynamic methods. Yamada et al. described a "pinched-flow fractionation" design that separates samples containing intact cells by size [117]. Smaller flowing particles tend to streamline closer to the wall of a channel. This tendency is used to separate sperm cells in a "pinched" chamber. As the sperm cells move closer to the wall, the chamber suddenly widens allowing the sperm cells and epithelial cells to flow in different directions. Hydrodynamic filtration operates on the same principle, but with perpendicular branched channels. The tendency of smaller sperm cells to move closer to 
the walls of the chamber allows for them to be directed into earlier branches with epithelial cells entering later branches for separate collection [36].

These methods alone may not be enough to obtain a clean separation, but improvements have been attempted through a combination of these techniques. The sample is injected into the device and separation of the cells begins with pinched-flow fractionation into branching channels. The collected sperm cells are then further separated through hydrodynamic filtration in perpendicular branching channels. A $50 \mu \mathrm{L}$ mock sample of 300 sperm cells/ $\mu \mathrm{L}$ and 1,000 epithelial cells/ $\mu \mathrm{L}$. In 30 minutes, sperm fraction containing 94\% male DNA was obtained [62]. The authors claim a simple and efficient design, but samples with higher epithelial to sperm cell ratios and lower cell concentrations need to be tested and free epithelial cell DNA in the sample would remain a problem.

Mixed sexual assault samples are among the most challenging evidence encountered in a forensic laboratory. The difficulty and time-consuming nature of evidence processing is one reason for a significant backlog in the processing of sexual assault kits (SAKs) [47]. New methods for differential extraction are sought after with higher throughput, fewer handling steps, and clearer suspect profiles.

\section{CHAPTER III. CELL CAPTURE TECHNIQUES}

\section{A. Introduction}

Magnetic cell separation incorporates magnetic particles conjugated to either antibodies or proteins. The application of a magnet to the sample allows for the selection 
and separation of tagged cells which may then be removed from others in a sample. The selection of cells of interest is a process known as positive selection. Negative selection is performed when the technique is applied for the direct removal of unwanted cells. The earliest magnetic separators utilized micrometer-sized Dynabead magnetic particles (Invitrogen, Carlsbad, CA). The first such separation performed for clinical purposes involved the negative selection and removal of gliomas from bone marrow required for a transplant [95].

\section{B. Sperm Cell Capture and Fertility Studies}

Research into this technique for the separation of sperm cells is ongoing for different applications. Fertility studies have led to the development of kits for increasing the viability of cryopreserved sperm cells through negative selection of apoptotic sperm cells from a sample. Apoptopic spermatozoa externalize the phospholipid phosphatidylserine (PS) making it accessible to magnetic particles with conjugated annexin V, a protein with an affinity for PS. These particles do not pass through the membrane and leave sperm cells with fertilization potential undisturbed [95].

\section{Sperm Cell Capture and Sexual Assault Evidence}

The positive selection of sperm cells from mixed case samples has been of great interest and could solve problems inherent in differential extraction methods. In 2008, Anslinger et al. attempted the separation of sperm cells using biomagnetic beads and monoclonal antibodies (mAbs) of the testicular isoform of the angiotensin-converting enzyme (tACE). Nine mAbs were investigated and three resulted in successful selection. These results were only achieved with samples preserved in phosphate-buffered saline 
(PBS) which preserved sperm cells with intact midpiece and flagellum, where the targeted antigen was more abundant. In dried samples, these sections may become detached from the sperm head, making this technique unsuitable for older samples [6].

Antigens located in the sperm head are needed for the viability of this technique. The motile sperm domain-containing protein 3 (MOSPD3) was considered as a solution to this problem. Experiments with both cotton swabs and nylon flocked swabs revealed $100 \%$ successful detection of alleles at each locus with day old samples. Unfortunately, success with the technique declined over time, resulting in successful detection of only $40 \%$ after 3 days, and $16.67 \%$ after 10 days for cotton swabs. Nylon flocked swabs showed superior performance with $87.5 \%$ successful detection after 3 days, and $40 \%$ after 10 days. In either case, the binding activity of the antigen was thought to have deteriorated over time [60].

The previous studies incorporating anti-MOSPD3 required 100,000 sperm cells with swabs containing 1,000 epithelial cells [60]. The study involving anti-tACE similarly used 100,000 sperm cells in a $1 \mathrm{~mL}$ buccal cell solution [6]. These results lead to an investigation for more potentially sensitive antigens. The sperm adhesion molecule 1 (SPAM1), also known as $\mathrm{pH}-20$, was considered for this purpose. This antigen, like MOSPD3, is located on the sperm head. Full STR profiles were reported in 9 of 10 samples with 1,000 sperm cells in a swab containing 100,000 epithelial cells. Unfortunately, in order to obtain these results, the procedure required the inclusion of a 30 minute DNase (Tiandz, Beijing, China) digestion step at $37^{\circ} \mathrm{C}$ and deactivation of the nuclease with EDTA at $65^{\circ} \mathrm{C}$ for 10 minutes. And this is preceded by 90 minutes of 
incubation with the anti-pH-20 immunomagnetic beads (IMBs). This procedure was also not tested over time as in the MOSPD3 study [118].

\section{Epithelial Cell Capture}

Epithelial cell capture kits also exist for purposes that include cancer research. Negative selection of these cells may be helpful in removing epithelial cells from sexual assault evidence. Stemcell Technologies produces the EasySep ${ }^{\mathrm{TM}}$ Human EpCam Positive Selection Kit (StemCell Technologies, Vancouver, Canada), an immunomagnetic cell capture kit that is designed for capturing human mammary epithelial cells. This kit can be used to capture excess female epithelial cells prior to pressure cycling. The selection cocktail in this this kit include 2 mouse IgG monoclonal antibodies [64]. One recognizes the epithelial cell adhesion molecule (EpCAM) that is present on normal epithelial cells, is overexpressed in solid cancers, and is involved in cell signaling, migration, and proliferation [102]. The other antibody attaches to dextrancoated magnetic particles. The cocktail also contains 2 rat anti-mouse IgG monoclonal antibodies that recognize the Fc-portion of mouse IgG. Together, they bridge the particles in the form of a tetrameric antibody complex [64]. 


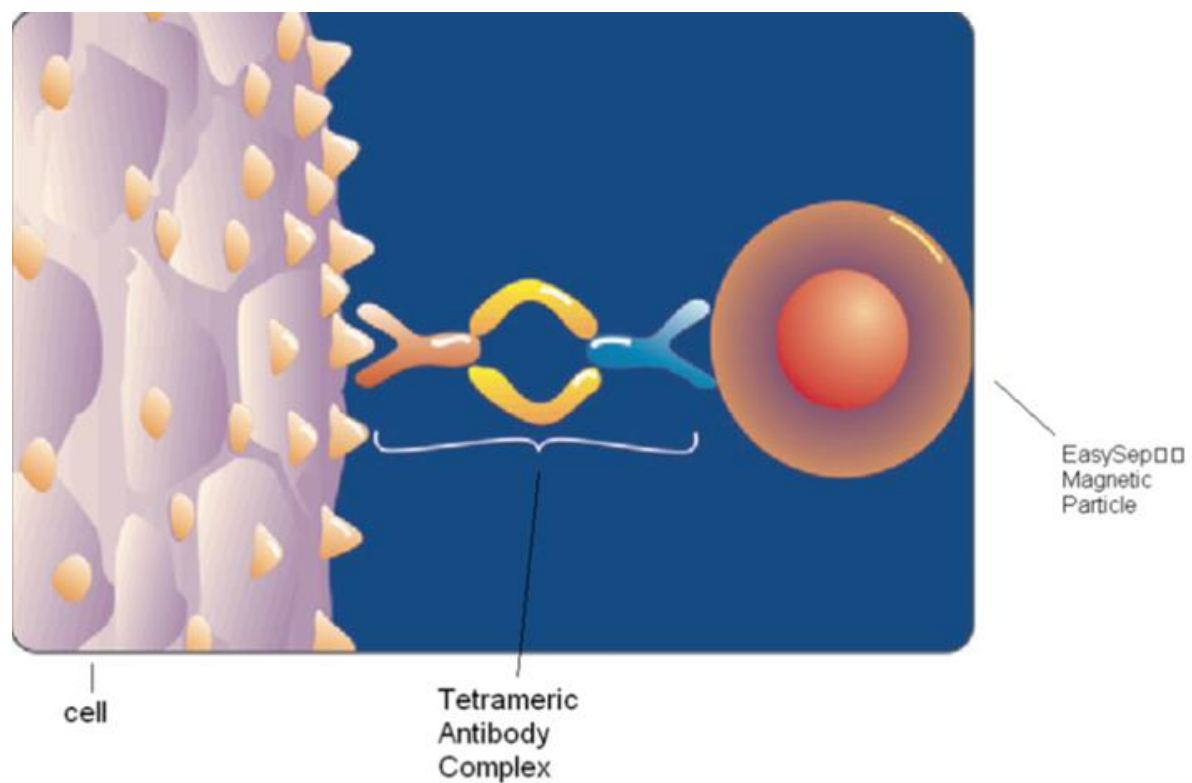

Figure 10. A tetrameric antibody complex is formed bridging the $\operatorname{EasySep}^{\mathrm{TM}}$ magnetic particle and the EpCAM antigen present on the cell. The rat ant-mouse IgG monoclonal antibodies are pictures in yellow. The mouse monoclonal antibody that recognizes EpCAM is pictured in red. The mouse monoclonal antibody that attaches to the dextran-coated magnetic particle is pictured in blue. Adapted from Stemcell Technologies official website.

Positive selection of sperm cells is a promising technique that is not currently ready for casework with older samples or samples with few sperm cells. Negative selection of epithelial cells may not be powerful enough to provide a clear suspect profile, especially with the likely presence of lysed epithelial cells and free DNA, but it may be useful as a pretreatment for samples prior to extraction

\section{CHAPTER IV. PRESSURE CYCLING TECHNOLOGY (PCT)}

\section{A. Introduction}

The application of cycles of high and low pressure have been shown to be more disruptive to cells than high pressure alone [81]. This mechanism of pressure-based lysis functions through the compression of cell membranes at high pressure. When the pressure 
is released, the disrupted cell membranes fly apart, releasing DNA, RNA, and other cellular debris from the sample [39].
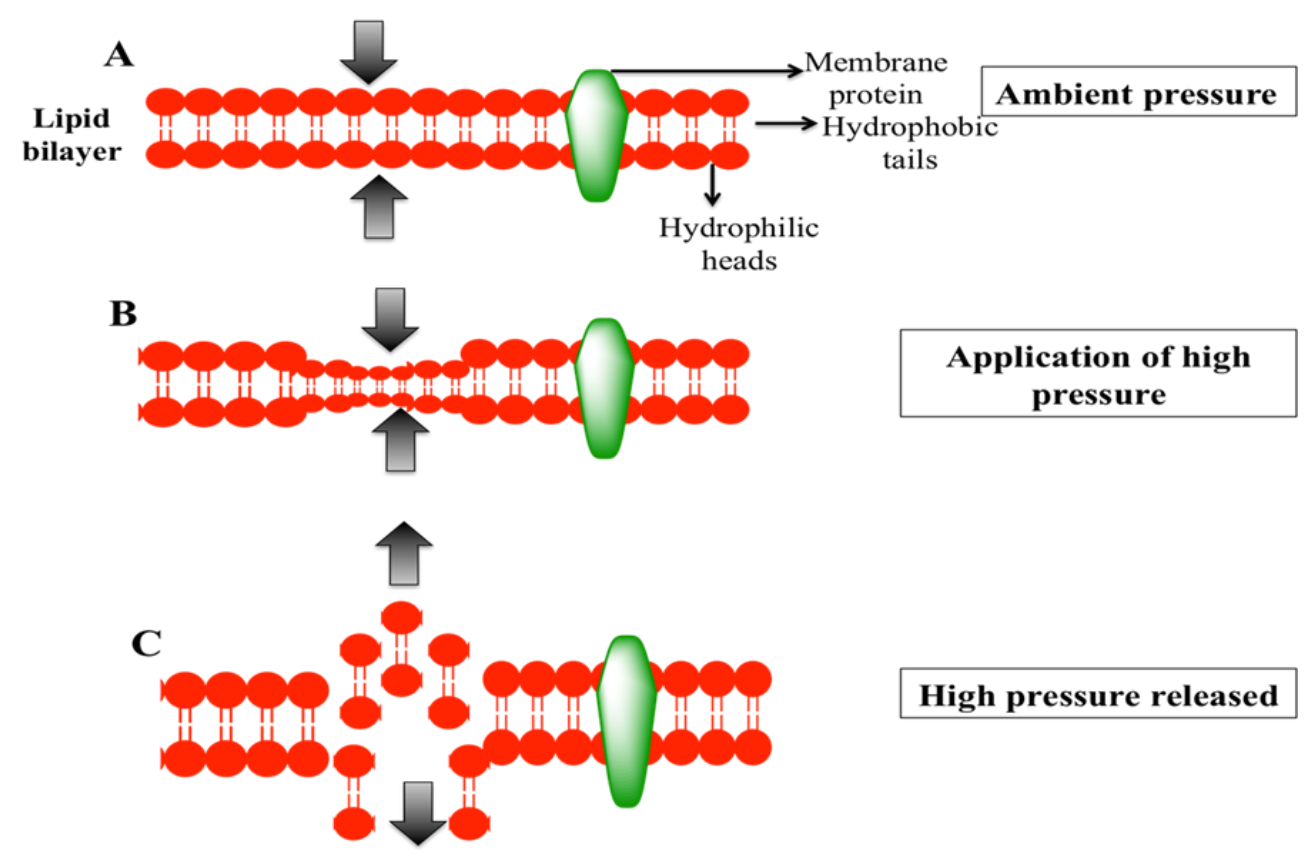

High pressure released

Figure 11. Mechanism of pressure based lysis. (A) The cell membrane at ambient pressure. (B) The cell membrane compressed by the application of high pressure. (C) The cell membrane breaks apart when the high pressure is released and the cell is lysed [39].

\section{B. Barocycler ${ }^{\circledR}$ NEP 2320 Instrument and Components}

The Barocycler ${ }^{\circledR}$ NEP 2320 (Pressure BioSciences Inc., South Easton, MA) is a lightweight pressure cycling technology (PCT) instrument that is compact enough to fit on a desktop. It contains a hydrostatic pressure chamber that is capable of achieving target pressures as high as 45,000 psi. A microprocessor and keypad are included to alter and save variables chosen by the user. These variables include target pressure, number of cycles up to 99 , and both time at target pressure and ambient pressure, which can be applied for 1-99 seconds. The Barocycler ${ }^{\circledR}$ NEP 2320 operates at temperatures from 4 $60^{\circ} \mathrm{C}$ that can be varied through connection to a circulating water bath. 


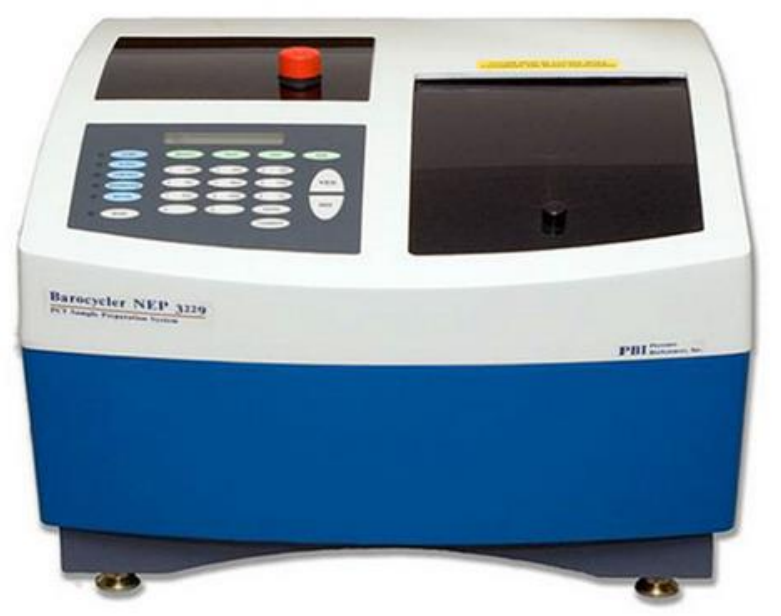

Figure 12. Barocycler® NEP 2320 from Pressure BioSciences, Inc. (South Easton, MA). Adapted from Pressure BioSciences, Inc. website.

PULSE $^{\text {TM }}$ tubes (Pressure BioSciences Inc., South Easton, MA) specifically made for Barocycler ${ }^{\circledR}$ instruments are capable of withstanding extreme pressures. The samples tubes include a moveable ram made of polypropylene on one end and are sealed with a screw cap on the other end. FT500 PULSETM tubes include a lysis disk intended for the disruption of solid tissues. The disks contain perforations that allow for sample to be pushed through into a fluid retention chamber filled with lysis buffer [32]. 


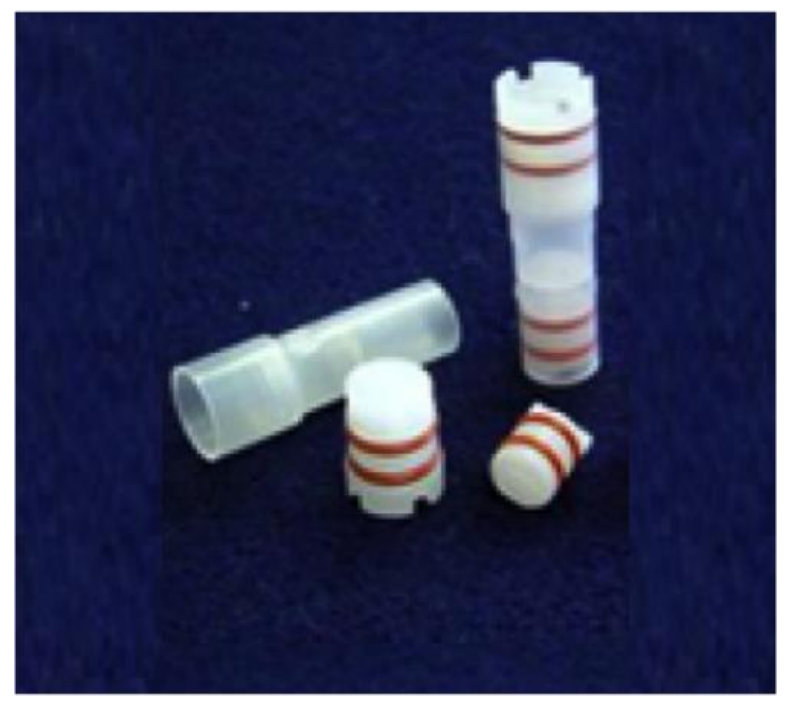

Figure 13. PULSE tube FT500. The ram transmits pressure to the sample and the screw cap seals the tube. The sample lies on top of the lysis disk filled with perforations that allow sample to be pushed into the collection chamber. Adapted for Pressure BioSciences, Inc. website.

FT500-ND PULSETM tubes without lysis disks are also available. These are best used for liquid samples and samples on solid substrates not intended for homogenization that contain absorbed liquid such as swabs or fabric [74]. As the pressure in the hydrostatic chamber differs from the pressure in the sample tube, the ram moves up to transmit pressure to the sample. When the pressure is released, the arm recedes. Repeated cycles of this process provide rapid and efficient release of nucleic acids, proteins, and other cellular contents from the sample [39]. The Barocycler ${ }^{\circledR}$ NEP 2320 can process one PULSETM tube at a time while the NEP 3239 model can handle up to three at once [74].

Up to 48 samples with volumes of $150 \mu \mathrm{L}$ or less can be processed at a time in microtubes placed in microtube cartridges. The tubes are made of fluoropolymer fluorinated ethylene propylene (FEP) with caps made of polytetrafluoroethylene that are manufactured to withstand high pressures and rapid pressure changes. Different caps are 
made for samples of 50,100 , and $150 \mu \mathrm{L}$ that form a tight seal to prevent bubble formation and sample loss from the tube [74].

\section{PCT for Difficult Sample Extraction}

There are many methods in use for traditionally hard-to-lyse tissues and cells such as bead-beating, manual grinding, enzymatic digestion, chemical dissolution, freezing with liquid nitrogen followed by grinding, sonication, and rotor-stator homogenization. These methods lyse cells and release nucleic acids and proteins for analysis, but may also lead to disruption of important complexes for analysis. Pressurebased lysis with FT500 PULSE ${ }^{\text {TM }}$ tubes may improve extraction by avoiding this disruption and also provide rapid and reproducible results. Problematic samples tested with this method include yeast, fungi, grape seeds, cardiac and skeletal muscle, breast tumors, and mosquitoes. Results have shown pressure cycling to yield better or comparable results when contrasted with traditional methods in shorter times and with more reproducibility [32].

\section{DNA Recovery from Soil Samples}

Extraction of microbial DNA from soil samples with pressure cycling has shown promising results. Studies done with extraction of agents of root rot, Rhizoctonia sulani AG-8 and Rhizoctonia oryzae, have reported a 16-fold and 2-fold increase in DNA yield respectively compared to bead-beating even with low population densities for these pathogens. Lyophilized wheat roots, which are known to be resistant to most homogenization methods, were also extracted with great reproducibility between results [78]. A different study recovered microbial genomic DNA from different types of soil 
samples with different extraction methods. While greater amounts of DNA were reported with vortex disruption and bead-beating, pressure cycling resulted in a greater number of unique terminal restriction fragments for analysis [18].

\section{E. Proteomic Analysis}

Pressure cycling has also been investigated as an extraction method for proteomic analysis. Current methods often involve time-consuming sample digestion and preparation steps. The protein, histone $\mathrm{H} 4$, was extracted for identification and quantitation in only 2 hours with pressure cycling at 15,000 psi with comparable results to enzymatic digestion with the proteolytic enzyme chymotrypsin for 18-24 hours of incubation [81].

Proteins from rat tissue including kidney, abdominal fat pad, liver, brain and cardiac muscle were extracted with pressure cycling and ProteoSolve-SB (Pressure Bio Sciences, Inc. South Easton, MA) with no homogenization or post-extraction clean up steps necessary [39]. Proteins from the nematode Caenorhabitis elegans were extracted with pressure cycling with yields $37 \%$ higher than sonication [99]. Escherichia coli protein yield from soil samples were reported as $14.2 \%$ higher than bead-beating [100]. Proteins extracted from liver tissue included those not isolated with other techniques. This and the previous soil sample study demonstrate the potential for unique data to be revealed with the use of this method [99]. 


\section{F. Inhibition}

It has been reported that pressure cycling could help overcome effects of known PCR inhibitors, humic acid and hematin, which are often encountered in blood and bone samples. Experiments were performed with and without DNA. DNA-free samples were evaluated by the internal positive control (IPC) included in the Quantifiler ${ }^{\text {TM }}$ Human DNA Quantification Kit (ThermoFisher Scientific Inc., Weston, FL) The presence of inhibitors tends to increase cycle threshold $(\mathrm{Ct})$ values. Samples processed with pressure cycling consistently showed lower Ct values than those processed without. STR analysis of samples containing DNA revealed higher relative fluorescence units (RFUs) when processed with pressure. Finally, powdered bone samples were incubated in extraction buffer at $56^{\circ} \mathrm{C}$ for 2 hours to overnight. Some samples were then processed with pressure cycling and others were not. Those that underwent pressure cycling treatment showed significantly higher RFUs and a greater number of detected alleles. The authors state that future work is needed to discover the mechanism behind these results [66].

Pressure cycling technology is a promising technique for the extraction of sexual assault evidence. Epithelial cells are more diffuse with a greater surface area than sperm cells making them more easily disrupted by pressure cycling [75]. The compact structure of sperm cells is the result of the presence of protamines in place of histones as in other cells. These positively-charged proteins attract the DNA allowing for a more compact and protected structure [80]. It may then be possible to lyse epithelial cells with the use of a Barocycler ${ }^{\circledR}$ instrument, leaving sperm cells behind to be extracted separately. 
Research must continue in order to further investigate the potential of this technique for differential extraction and other forensic applications.

\title{
CHAPTER V. DNA ANALYSIS
}

\section{A. STR Analysis and Other Genetic Markers}

\begin{abstract}
A.1. STRs
Short tandem repeats (STRs), also known as microsatellites, are regions in the genome that contain repeat units that range from 2-7 base pairs (bp) in length. STRs are the most commonly used genetic markers for current forensic applications. Selected genetic markers show high polymorphism and the short sequences are useful for degraded evidence which contain highly fragmented DNA. STRs make up about $3 \%$ of the human genome making them relatively common [24].

Different alleles are distinguished by the number of repeat units found at the locus. Heterozygous individuals have a different number of repeats on each chromosome whereas homozygous individuals have the same number on each [15]. There are several different types of STRs that vary according to repeat unit. Simple repeats are all of identical length and sequence. Compound repeats consist of two or more simple units adjacent to one another. Complex repeats are variable in both length and sequence. Microvariants also exist that contain incomplete repeats. For example, the 9.3 allele of the TH01 locus contains 9 repeats 4 base pairs long and one repeat with only three base pairs [24].
\end{abstract}


STRs are also characterized by repeat length. Dinucleotides consist of two repeats, trinucleotides have three, tetranucleotides have four, and so on. Tetranucleotides are the preferred length due to better resolution of different alleles than shorter repeats [92] as well as lower amounts of stutter products These products are formed by strand slippage of the template DNA in which a repeat is missed and not amplified. Longer repeat units such as pentanucleotides and hexanucleotides may also be selected as genetic markers, but are not as common as tetranucleotides [24].

Primers, short strands of DNA that serve a starting points for DNA synthesis, are created to target conserved sequences that flank the STR sequence of interest. The design of such primers as well as validation studies for their use can take up much time and resources. Commercial kits are available that save on time and conveniently allow for standard results that can allow for reproducible data between different laboratories. These kits frequently include master mix with polymerase, deoxynucleotides (dNTPs), and buffer as well as a primer mix, size standard, allelic ladder, and positive control DNA [24].

\section{A.2. Multiplex Kits}

Multiplexing is the inclusion of two or more primer sets in a reaction. These primers must be carefully chosen to avoid excessive complementarity that can lead to primers annealing to each other to form primer dimers and should be otherwise compatible with similar annealing temperatures. Amplification products are detected through fluorescence of dyes used to label primers. Different primers can be labeled with different colored dyes to easily resolve alleles from distinct loci. Loci labeled with the 
same dye must have adequate size separation between amplification products from other loci for appropriate detection and identification [24].

Size standards are commonly labeled with a unique dye for detection in a separate lane to distinguish the peaks from true alleles and allow for determination of PCR product size based on comparison of electrophoretic mobility [35].

Allelic ladders contain all common alleles that may be encountered at each locus included in a kit. The primers included in the primer mix are also used to generate the ladder in order to ensure proper alignment and identification since the alleles in the ladder will migrate the same distance as alleles encountered in samples [24]. These ladders can be used to detect shifting of results attributable to instrumentation, environmental condition or differences in polymer [22].

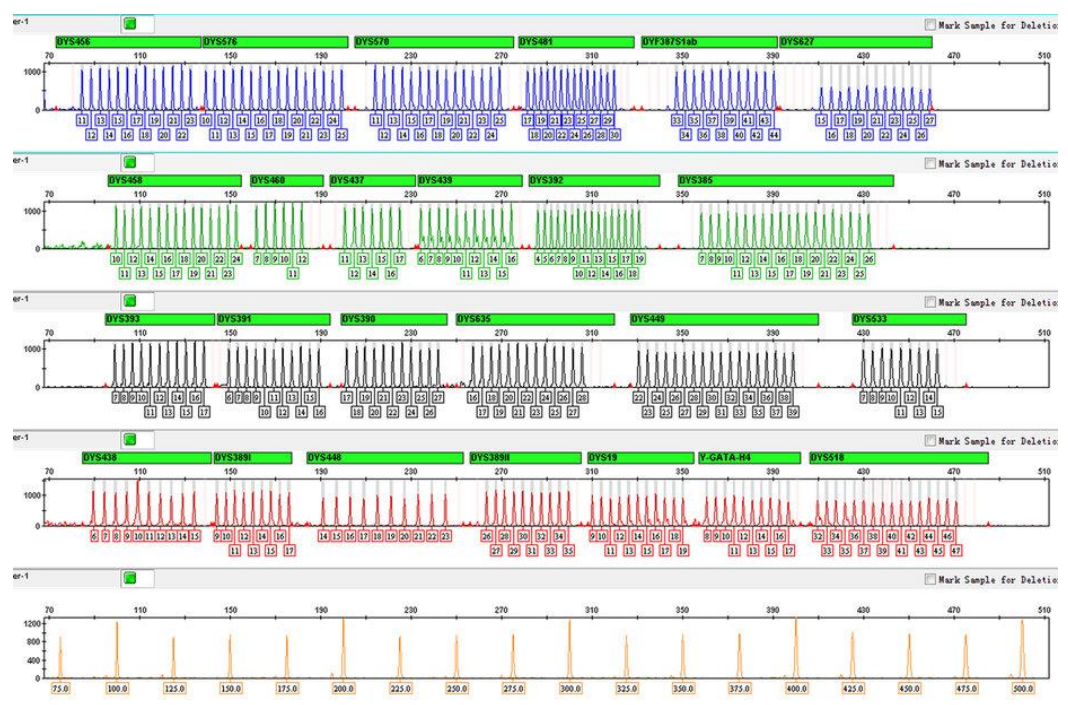

Figure 14. Allelic ladder and internal size standard in the Y-STR STRtyper-27 system. The top four panels display the allelic ladder. The represented locus is labeled in the green bar above each panel and all common alleles are represented by the peaks below. The bottom panel displays the internal size standard labeled in an orange dye [8]. 


\section{A.3. CODIS Loci}

Beginning in 1996, there was a FBI Laboratory sponsored endeavor to establish core loci to be included in the national DNA database known as the Combined DNA Index System (CODIS). These 13 core loci were determined to be, CSF1PO, D3S1358, D5S818，D7S820，D8S1179，D13S317，D16S539，D18S51，D21S11，FGA，TH01, TPOX, and vWA [41]. Commercial kits include these loci as well as the sex determination marker Amelogenin, In order to further improve power of discrimination, even more loci are included with some, such as Powerplex Fusion ${ }^{\circledR}$ System (Promega Corporation, Madison, WI) incorporating as many as 24 in a single multiplex [88]. As of January $1^{\text {st }}$, 2017, 7 more loci will be required for inclusion in the National DNA Index System (NDIS) D1S1656, D2S441, D2S1338, D10S1248, D12S391, D19S433, and D22S1045 [41].

\section{A.4. Y-STRs}

Y-STRs are male specific genetic markers located on the non-recombining portion (NRY) that makes up 95\% of the Y chromosome [24]. As recombination does not occur, the haplotype is passed down from father to son unchanged except for instances of mutation [4]. As many people such as family members from the same patrilineal line, may have the same haplotype these markers lack the power of discrimination of autosomal STR markers [22]. These markers are still very useful in cases of sexual assault mixed male and female DNA where a clear suspect genotype cannot be resolved either

due to a lack of intact sperm or sample being overwhelmed by the DNA of a female victim 
[24]. Separate kits are available for Y-STR identification such as in the Yfiler ${ }^{\circledR}$ Plus DNA Amplification Kit (ThermoFisher Scientific Inc., Weston, FL) [84]. They may also be included with autosomal STRs such in PowerPlex Fusion ${ }^{\circledR}$ 6C (Promega Corporation, Madison, WI). This kit contains primers for 23 autosomal STR loci, Amelogenin, and 3 Y-STRs for a total of 27 loci with 6 dyes [87].

\section{A.5. Mitochondrial DNA}

Mitochondrial DNA is passed down from mother to children of either sex with no recombination although, as with Y-STRs, mutations may occur [13]. It has been considered that paternal mtDNA may be diluted by excessive amounts of mtDNA in an oocyte, or that paternal mtDNA may not enter the oocyte at all. But recent evidence suggests that nucleases present in the oocyte selectively degrades paternal mtDNA making inheritance uniparental [96]. Unlike genomic DNA, mtDNA is not contained as a pair of copies inside a nucleus. Instead, multiple copies of mtDNA can be found in each cell [93]. This makes mtDNA invaluable in cases of degraded evidence such as skeletal remains or in cases where little genomic DNA may be recovered as in hair shaft evidence. Identification of victim remains can also be conducted through comparison with DNA from matrilineal relatives [112].

\section{B. Polymerase Chain Reaction (PCR)}

\section{B.1. Introduction}

In 1985, Kary Mullis described the polymerase chain reaction (PCR) in which multiple copies of a DNA sequence can be generated from even trace amounts of sample 
[24]. The reaction proceeds with the addition of several reagents and applied cycles of rapidly changing temperatures. This technique has become indispensable to forensic applications and all areas of molecular biology [35].

\section{B.2. PCR Reaction Steps}

The PCR process involves three main phases; denaturation, annealing, and extension. These phases occur according to rapid alterations in temperature provided by a thermal cycler instrument. The denaturation step occurs at temperatures of more than $90^{\circ} \mathrm{C}$ in order to break apart the hydrogen bonds that hold together the nitrogenous bases. The results in the denaturation or melting of dsDNA into ssDNA.

After denaturation, the temperature is lowered to anywhere between $40-60^{\circ} \mathrm{C}$ for the annealing phase. The temperature at this step depends on the annealing temperature of the included primers. These primers bind to the complementary sequences at on the template DNA. These sequences are conserved regions flanking the sequence selected for amplification.

The final step is the extension phase. The temperature is raised to $72^{\circ} \mathrm{C}$, the optimal temperate for DNA polymerase functioning. This enzyme incorporates deoxynucleotide triphosphates (dNTPs) along the DNA strand forming a complementary strand for the target sequence [29].

Ideally, the products of each cycle are 2 dsDNA strands for each single strand present in the reaction. This increases the amount of DNA exponentially, with $2 \mathrm{n}$ copies produced with for each cycle "n" [24]. 


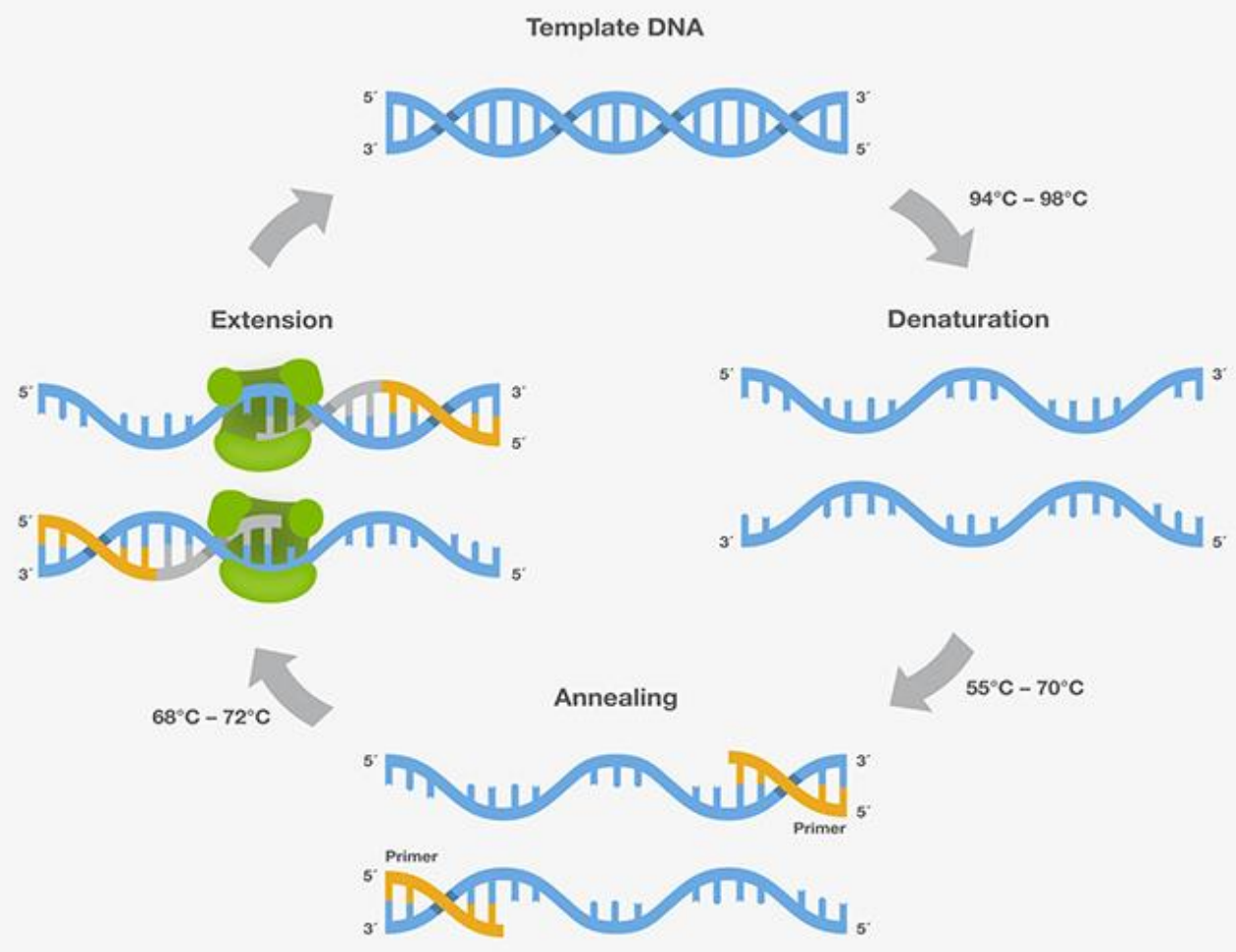

Figure 15. The PCR process includes three steps; denaturation, annealing, and extension. The cycle then repeats for exponential amplification. Adapted from ThermoFisher Scientific website.

\section{B.3. PCR Components}

The components of a PCR reaction include primers, DNA polymerase, dNTPs, template DNA, and buffer. Selected primers should not only bind to conserved regions of the target sequence, but should also be designed to avoid self-complementarity in order to prevent the formation of unwanted products such as primer dimers and hairpins that can reduce the efficiency of the reaction [35]. 
The building blocks needed for new DNA sequences strands are dNTPs; nucleotide molecules with 3 phosphate groups. PCR reactions include equal measure of dNTPs with each of the four bases; dATP, dCTP, dGTP and dTTP [50].

DNA polymerase is an enzyme that binds to the primer sequence and generates a DNA strand through the incorporation of dNTPs. Earlier PCR attempts required continuous replenishment of DNA polymerase as it would be deactivated by high temperatures needed during the denaturation step. The introduction of polymerase from the thermophilic bacteria Thermus aquaticus (Taq) revolutionized this process and allowed for greater automation as this polymerase remained stable at $95^{\circ} \mathrm{C}$ [50].

The amount of DNA template added to each reaction must be carefully controlled as too much can lead to allele drop in and too little can lead to allele drop out. Artifacts such as pull up or stutter peaks may also occur. Care must be taken to prevent contamination as results may be complicated by the amplification of DNA sequences other than those of the template [35].

Other components include buffer for stability of the reaction as well as $\mathrm{MgCl}_{2}$ as a co factor for the proper functioning of polymerase [35]. Nuclease-free deionized water is added at an appropriate volume to prevent evaporation of sample [29].

\section{B.4. Thermal Cyclers}

PCR reactions are run on thermal cycler instruments that are capable of rapid heating and cooling using Peltier systems often with silver or gold plated blocks that allow for quickly changing temperatures and more uniform heating of samples. These 
instruments can be programmed with number of cycles, temperature at each step and ramp speed determined by the user [35].

Amplification often requires hours with a traditional thermal cycler due to lower heating and cooling rates. Rapid PCR amplification can be accomplished using instruments with Peltier systems capable of high heating and cooling rates such as the Philisa ${ }^{\circledR}$ from Streck Inc. (Nebraska, US). This instrument is capable or heating and cooling rates of $15^{\circ} \mathrm{C} / \mathrm{s}$ and $10^{\circ} \mathrm{C} / \mathrm{s}$ respectively where traditional instruments can have rates as low as $5^{\circ} \mathrm{C} / \mathrm{s}$ for each. Instruments such as these can shorten amplification times to less than 15 minutes [2].

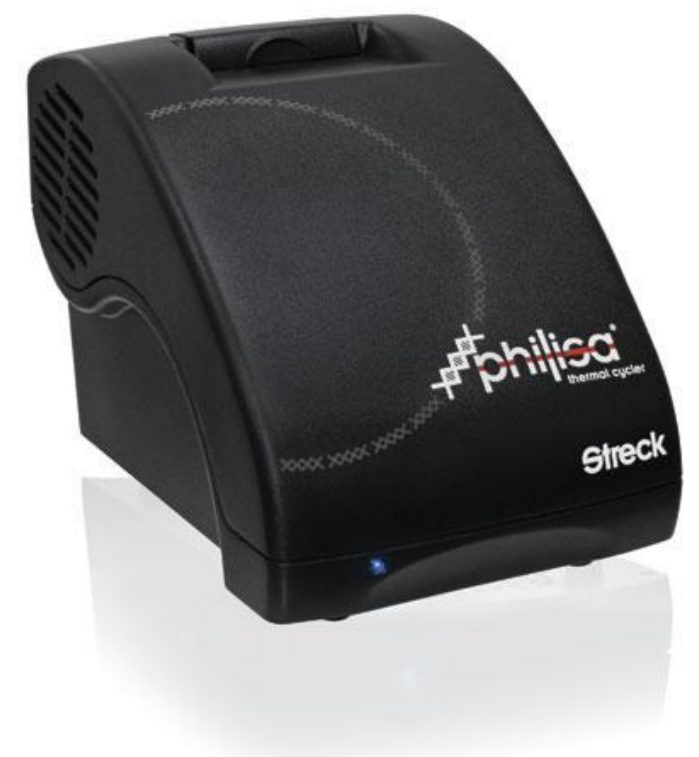

Figure 16. Philisa ${ }^{\circledR}$ Thermal Cycler from Streck, Inc. (Nebraska, US). Adapted from Streck, Inc. website.

\section{B.5. Real-time PCR}

Quantification of DNA in a sample is necessary for optimal functioning of a PCR reaction. PCR reactions can be monitored in real-time as DNA is amplified. Real-Time 
PCR or quantitative PCR (qPCR) allows for quantification through a change in fluorescence. As the reaction proceeds, the concentration of DNA ideally doubles with each cycle during what is known as the exponential phase. As reagents in the reaction are expended, the reaction slows down and the linear phase begins. Eventually, the reagents are all taken up by the reaction and the plateau phase is reached. There is a point in the reaction where the fluorescent signal exceeds that of the background level. The cycle where this "threshold" is reached is known as the cycle threshold $(\mathrm{Ct})$ [25]. More concentrated samples reach this threshold sooner. Quantification is determined according to the $\mathrm{Ct}$ of a sample reaction and through comparison with a generated calibration curve through the inclusion of standards of known concentration [35].

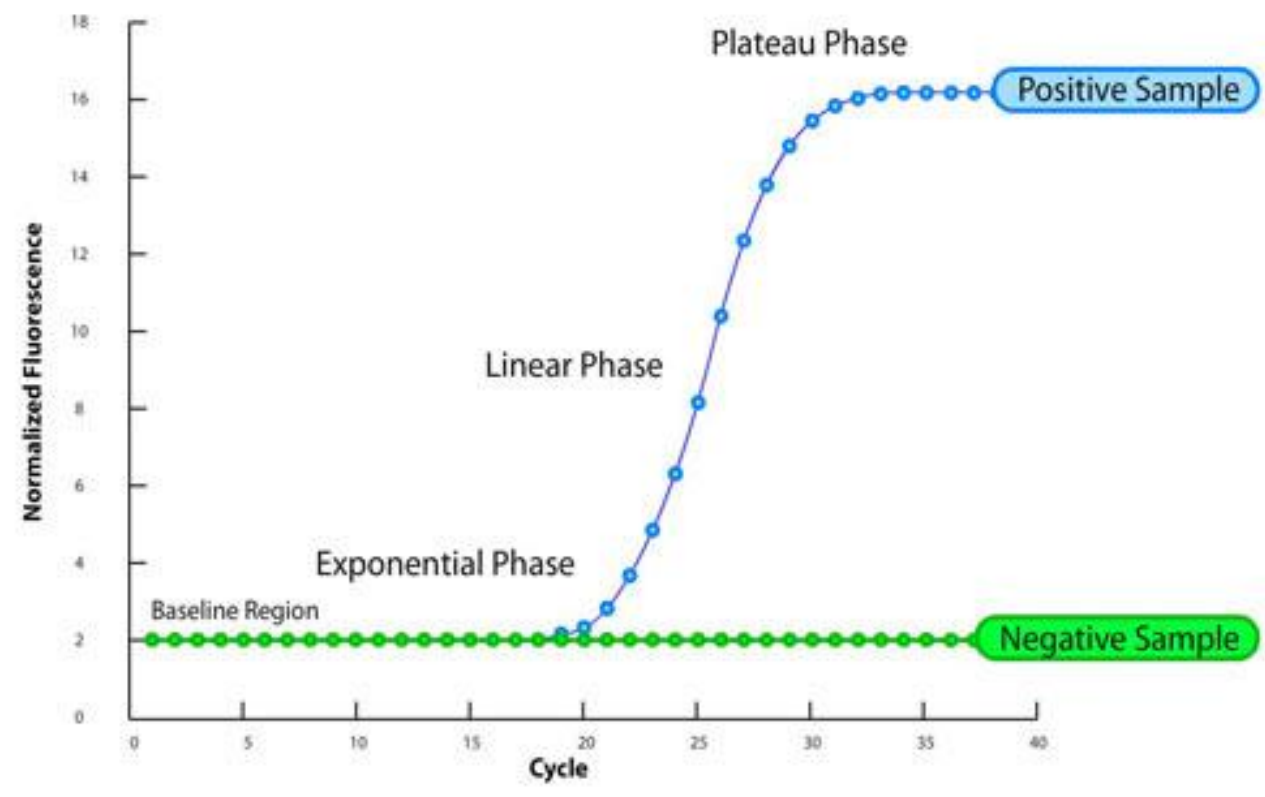

Figure 17. Phases included in a qPCR reaction; exponential phase as product doubles each cycle, linear phase as reagents are depleted and reaction no longer proceeds exponentially, and the plateau phase is entered as regents are exhausted. Adapted from Abbot Molecular website. 
The first such technique utilized ethidium bromide which fluoresces when it is intercalated in dsDNA. SYBR ${ }^{\circledR}$ green is an intercalating dye that functions in the same way [35]. TaqMan ${ }^{\circledR}$ takes a different approach. A probe is included in the reaction with a fluorescent molecule on the 5' end and a quencher molecule on the 3' end. The probe anneals to the DNA template and as DNA polymerase extends, the enzyme cleaves the probe in its path through exonuclease activity and releases the fluorophore from the quencher allowing for its fluorescence to be detected. As more DNA is amplified, more probes are cleaved, and more fluorescence is detected [46].

Plexor $^{\circledR}$ HY System uses a different method that relies on modified nucleotides that pair only with each other; isoguanine (iso-dG) and 5'-methylisocytosine (iso-dC). One primer contains an iso-dC residue and a fluorescent label on the 5' end. The modified iso-dGTP nucleotide includes a Dabcyl quencher. When it is incorporated opposite iso$\mathrm{dC}$ residue the fluorescent label is quenched. As the reaction proceeds fluorescence decreases [55]. 


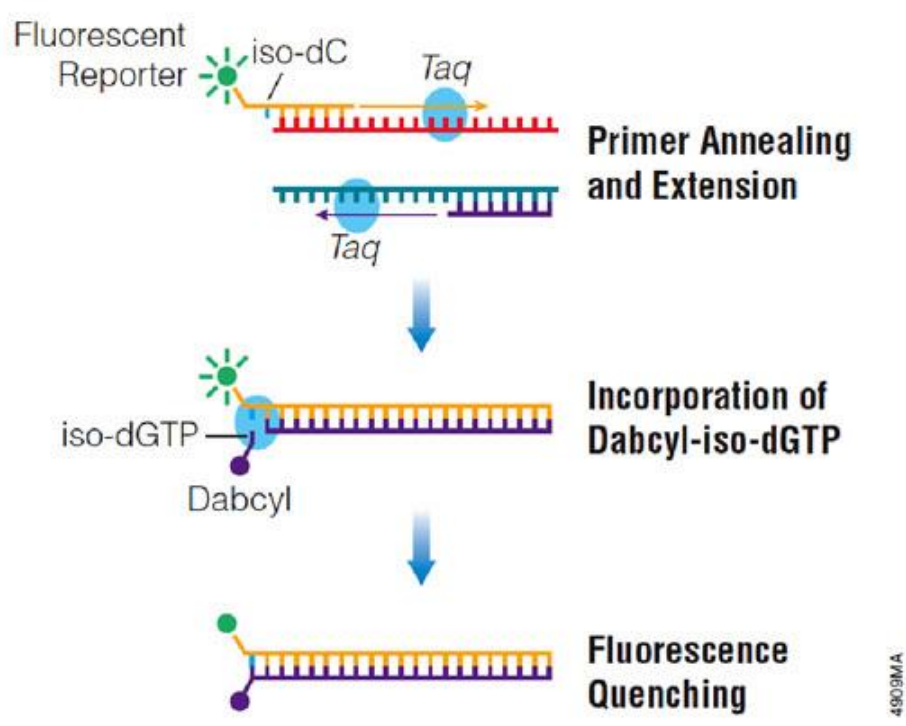

Figure 1. Quenching of the fluorescent signal by dabcyl during product accumulation.

Figure 18. As the extension step proceeds, the Dabcyl-iso-dGTP nucleotide is incorporated opposite the fluorescent reporter and fluorescence is quenched. Overall fluorescence in the reaction decreases as product increases. Adapted from Promega website.

\section{B.6. PCR Inhibitors}

Evidence recovered from a crime scene often includes substances that can interfere with a PCR reaction. Inhibitors than are capable of binding to DNA reduce available template and lead to poor recovery [68]. Other inhibitors may bind to polymerase and decrease the efficiency of the enzyme. Unfortunately, many biological materials are known to have inhibitory effects. Hemoglobin from blood, calcium or collagen from bone, bile salts from feces, melanin from hair or skin can all lead to inhibition. Samples found in soil can be contaminated by inhibitors such as humic acid or tannic acid. Indigo dye found in denim is another known inhibitor. It is often preferable to swab evidence from a sample deposited on this material rather than processing a cutting to decrease the inhibitor's effects [19]. Dilution of a sample can also reduce inhibition 
and such effects can be monitored with the inclusion of an internal positive control (IPC) included in a reaction $[4,82]$.

\section{Capillary Electrophoresis}

\section{C.1. Introduction}

Once amplification is complete, the generated products are present in the form of DNA fragments of various lengths labeled with fluorescent dyes. In a multiplex reaction, these fragments are products from several loci and different fluorescent dyes may be used for better resolution and simplified detections. Capillary electrophoresis (CE) is most often used for separation of these fragments and subsequent detection of alleles. The instrument includes a narrow, glass capillary filled with entangled polymer and 2 buffer vials and electrodes connected to a high voltage power source situated at either end of the capillary. Instruments such as the ABI PRISM ${ }^{\circledR} 310$ include a single capillary allowing for only 1 sample processed at a time. Newer models with capillary arrays such as the 3100 and 3130xl can run 16 samples at once while the 3700 can run 96 [35]. 


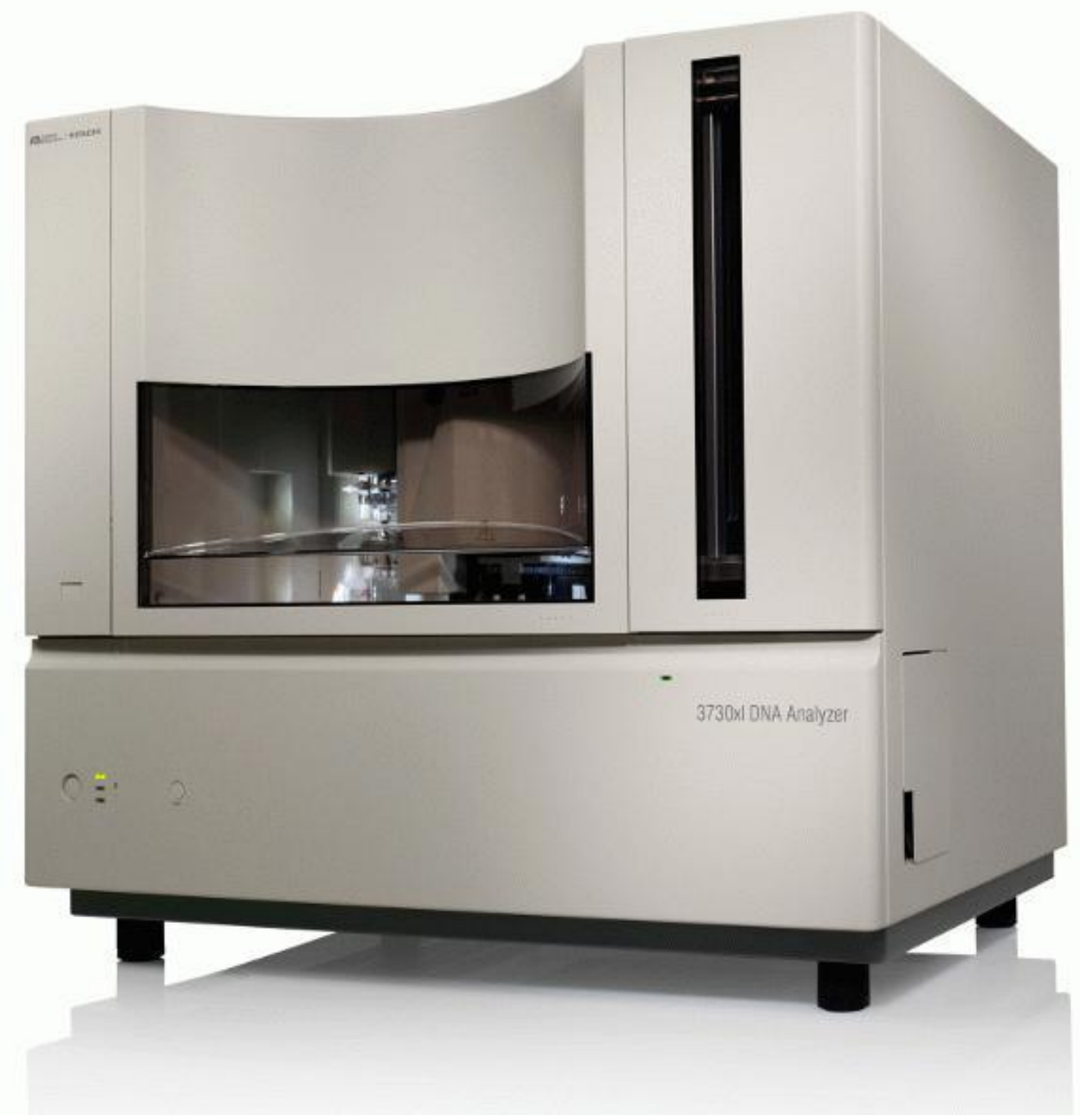

Figure 19. 96-capillary 3730xl DNA Analyzer from ThermoFisher Scientific (Weston, FL). Adapted from ThermoFisher Scientific website.

\section{C.2. Sample Preparation}

Samples are prepared in 10-20 $\mu \mathrm{L}$ of deionized formamide in order to denature the dsDNA into single strands [21]. Snap cooling, in which the sample is heated to $95^{\circ} \mathrm{C}$ then cooled to $4^{\circ} \mathrm{C}$ also aids in denaturation. Deionized water in conjunction with snap cooling can also be used instead of formamide, but reannealing of the single strands is a 
possibility [12]. The internal lane size (ILS) standard is also added at this point. An allelic ladder prepared in the same fashion as a sample is often run in a separate well [35].

\section{C.3. Capillary}

Capillaries are made of fused silica and coated with polyacrylamide for durability. For forensic applications, the capillary is usually $47 \mathrm{~cm}$ long with an internal diameter of only $50 \mu \mathrm{m}$ [24]. A window is burned into the polyacrylamide coating to create a detection window. An argon laser strikes the sample through this window for excitation of the fluorescently labeled DNA molecules. The emitted fluorescence is first filtered to reduce background noise and detected by a charged coupled device (CCD) camera. The amount of detected PCR product is proportional to the peak height measure in relative fluorescent units (RFU) [35].

Joule heating occurs when a current passes through a resistive medium and can degrade gel or polymer used in electrophoresis. The narrow capillary has a high surface area to volume allowing for greater heat dissipation and the application of a strong electric filed which increases mobility and decreases separation time. A balance must be struck between speed and resolution as the former can decrease the latter [98].

Silanol groups on the walls of the capillary are ionized at a $\mathrm{pH}>5$ giving them a negative charge. This attracts positively charges molecules in the buffer to the walls of the capillary and an electric double layer forms with one remaining fixed to the wall and the outer layer dragging the bulk of the solution towards the negatively charged cathode resulting in what is known as electroosmotic flow (EOF). The EOF is undesirable for 
many applications and can be suppressed with the introduction of polymer that coats the capillary walls [98].

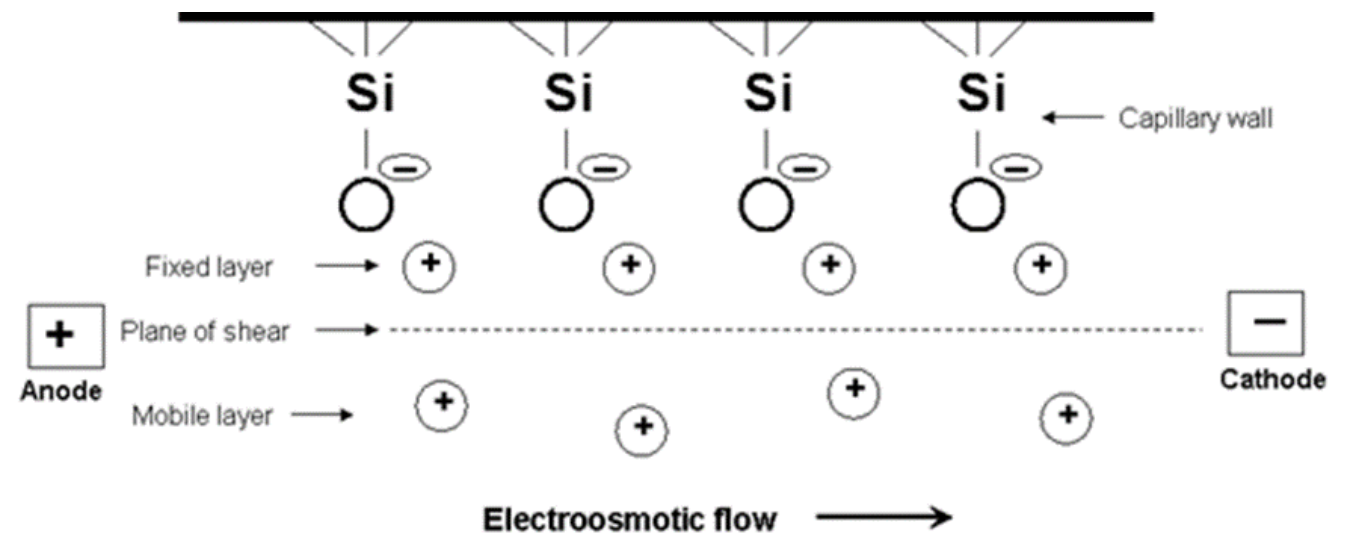

Figure 20. At pH > 5, the silanol groups become ionized creating an electric double layer composed of cations. An electroosmotic flow results in which all molecules are dragged toward the negatively charged cathode regardless of charge [37].

\section{C.4. Polymer}

The entangled polymer acts as a sieving matrix that acts as an obstacle for DNA molecules passing through the capillary and separating them by size. Larger molecules are hindered whereas smaller molecules pass through more easily. There are two models suggested to better explain this process. In Ogston sieving, the DNA is thought to pass through as a rigid, spherical molecule with larger molecules having a greater radius of gyration making it more difficult for such DNA fragments to pass through the mesh as a rigid particle. According to the reptation model, the linear molecules pass though pores in the polymer as a single strand with longer strands taking a longer time to unwind and navigate the entangled network [22].

Urea is included in the polymer in order to maintain the denatured DNA strands and avoid the formation of secondary structures that may impede separation or complicate 
results. Separations are generally performed at high temperatures to further maintain denaturation and to decrease separation time. For this reason, entangled polymers are prepared with thermal stability in mind. For the purposes of STR analysis, polymer concentration is prepared with $4 \%$ dimethyl polyacrylamide with higher concentrations used for other applications such as DNA sequencing [24].

\section{C.5. Buffer}

The buffer used most commonly in CE is $100 \mathrm{mM}$ 3-[[1,3-dihydroxy-2(hydroxymethyl)propan-2-yl]amino]propane-1-sulfonic acid (TAPS), with 1mM with ethylenediaminetetraacetic acid (EDTA) buffer. The buffer controls the $\mathrm{pH}$ and ionic strength in the capillary. Usually, the buffer is adjusted to a $\mathrm{pH}$ of 8 with sodium hydroxide which allows for the ionization if the silanol groups on in the capillary [35].

Electrokinetic injection introduces sample into the capillary. The application of a positive voltage causes the negatively charged DNA molecules to enter the capillary. The voltage and injection time influences the amount of DNA that is introduced. Ionic strength of the buffer can affect the amount of sample introduced. Negatively charged ions in the buffer, such as chlorine ions, can compete with DNA for introduction into the capillary [24].

\section{C.6. Data Interpretation}

Software such as GeneMapper ${ }^{\circledR}$ ID software (Applied Biosystems, Valencia, CA) or GeneScan ${ }^{\circledR}$ (Applied Biosystems, Valencia, CA) are designed for interpretation of the collected data. As there are dyes of different colors included in these reactions, the 
software must implement spectral calibration to separate the different colors into distinct lanes for ease of interpretation. The software determines the amount of spectral overlap between each dye and subtracts this from the peaks within the profile. Matrix standards are used to generate matrix profiles that contain the information necessary for successful calibration [35].

The ILS standard contains DNA fragments of known length used for accurate sizing of the DNA fragments. A size calling curve is generated by the interpretation software and the data from unknown fragments in the sample are compared with this size calling curve. Developed algorithms are used to measure the size of DNA fragments with the most common being the local Southern method [24].

\section{C.7. Artifacts}

Artifacts can complicate the analysis of a generated profile. Stutter peaks are one repeat smaller than the true allele that result when a repeat is skipped over by the polymerase in an event refers to as strand slippage occurs. Occasionally forward slippage may occur that results in peaks one repeat longer caused by a repeat transcribed twice. Fortunately, stutter peaks tend to less than $15 \%$ and setting threshold limits aids in interpretation as these peaks are be disregarded.

Taq has a property called terminal transferase which results in the addition of a nucleotide, usually adenine, at the end of an amplified product. Too much template can lead to incomplete adenylation with some products lacking the extra nucleotide. These split peak artifacts are $1 \mathrm{bp}$ shorter than the true peak and can appear as a "shoulder" beside the true peak if the majority of the strands contain the extra nucleotide. 
A poor quality matrix can result in poor spectral calibration and peaks of more than one color. These can appear in the generated profile as a pull up artifact. They will appear at the same size as a true peak at a different color lane. Overloading of a sample can also result in pull up regardless of the quality of the profile [35].

VI: OPTIMIZATION OF PRESSURE CYCLING AND ALKALINE LYSIS

\section{PROTOCOL}

\section{A. Introduction}

There is currently a backlog of sexual assault kits (SAKs) and other forensic evidence awaiting analysis in the United States. It is unknown how many remain unanalyzed nationwide [119], although some estimates have set the number as high as 500,000 [47]. Census studies have been conducted in major cities in order to accurately determine the extent of this backlog. Reports indicate the discovery of 8,707 and 6,663 SAKs that have never been submitted for analysis in Detroit and Houston respectively. Many other kits of unknown status have also been reported [119].

One factor that stalls evidence processing is the extraction method itself. Conventional differential extraction involves an initial step for the lysis of female epithelial cells in the sample with sodium dodecyl sulfate (SDS) and Proteinase K [33]. After incubation and centrifugation, the supernatant containing the female DNA is removed. The sperm pellet left behind is washed to remove residual female DNA and lysed with the addition of Dithiothreitol (DTT) to reduce the disulfide bonds in the sperm cell that make it resistant to organic extraction. This process can be time-consuming, laborious, difficult to automate, and often results in poor recovery of DNA from the swab 
used to collect the sample [111]. The female DNA or undigested epithelial cells may also be left behind in the sperm cell pellet leading to a mixed genotype. The necessary number of washings and centrifugations during this process is determined by the examiner. This makes results dependent on user expertise.

Research has been conducted regarding the development of a novel method for differential extraction involving pressure cycling technology (PCT) and alkaline conditions for cell lysis. Pressure-based lysis can be achieved with the use of the Barocycler $^{\circledR}$ NEP 2320 from Pressure Biosciences, Inc. (South Easton, MA). This instrument lyses cells by applying cycles of ambient and high pressure to the sample contained in a specially designed PULSETM tube (Pressure Biosciences, Inc., South Easton, MA) that can withstand high pressures. The tube includes a movable ram that transmits pressure to the sample. The hydrostatic chamber of the Barocycler ${ }^{\circledR}$ is capable of pressures ranging from 5-45k psi. The number of cycles, time held at high or ambient pressure, and temperature are all parameters that can be adjusted by the user [74].

The pressure cycling step tends to selectively lyse epithelial cells rather than sperm cells due to differences in cell structure. Lipids are compressed by high pressure and are disrupted when that pressure is released. The epithelial cells are larger and more diffuse which allows them to be more easily disrupted by pressure than the compact sperm cells [74]. Most DNA is wrapped around histone proteins. In sperm cells, these proteins are mostly replaced by protamines. The positive charges in this protein allow the negatively charged DNA to be packed more tightly and protected from the environment [80]. 
Alkaline lysis for the purpose of differential extraction has been described for the improved recovery of sperm cells, which have a tendency to adhere to the substrate on which they are collected. The epithelial cells are lysed at high temperatures in a $0.1 \mathrm{~N}$ $\mathrm{NaOH}$ solution. After DNase digestion of the female DNA, alkaline lysis of the sperm cells is accomplished at a lower temperature and with a $1 \mathrm{~N} \mathrm{NaOH}$ solution. This conveniently also denatures and inactivates the DNase enzyme [49].

A two-step protocol has been developed that uses pressure-based lysis in alkaline conditions for the removal of epithelial cells followed by alkaline lysis at high temperatures for the recovery of the sperm fraction. The flow chart for the protocol is depicted in Figure 21. The first step requires the swab to be suspended in $0.4 \mathrm{~N} \mathrm{NaOH}$. It is then processed in the Barocycler for 10 cycles, with 15 seconds at 20,000 psi followed by 15 seconds at ambient pressure for a total of 5 minutes to recover the epithelial cell fraction. The solution is neutralized with the addition of $2 \mathrm{M}$ Tris ( $\mathrm{pH}$ 7.5). After centrifugation, the swab is then suspended in $0.4 \mathrm{~N} \mathrm{NaOH}$ for another 5 minutes at $95^{\circ} \mathrm{C}$. The solution is once again neutralized with $2 \mathrm{M}$ Tris $(\mathrm{pH} 7.5)$ and centrifuged. After a total of 20 minutes, both the epithelial and sperm fractions are ready for purification [74]. 


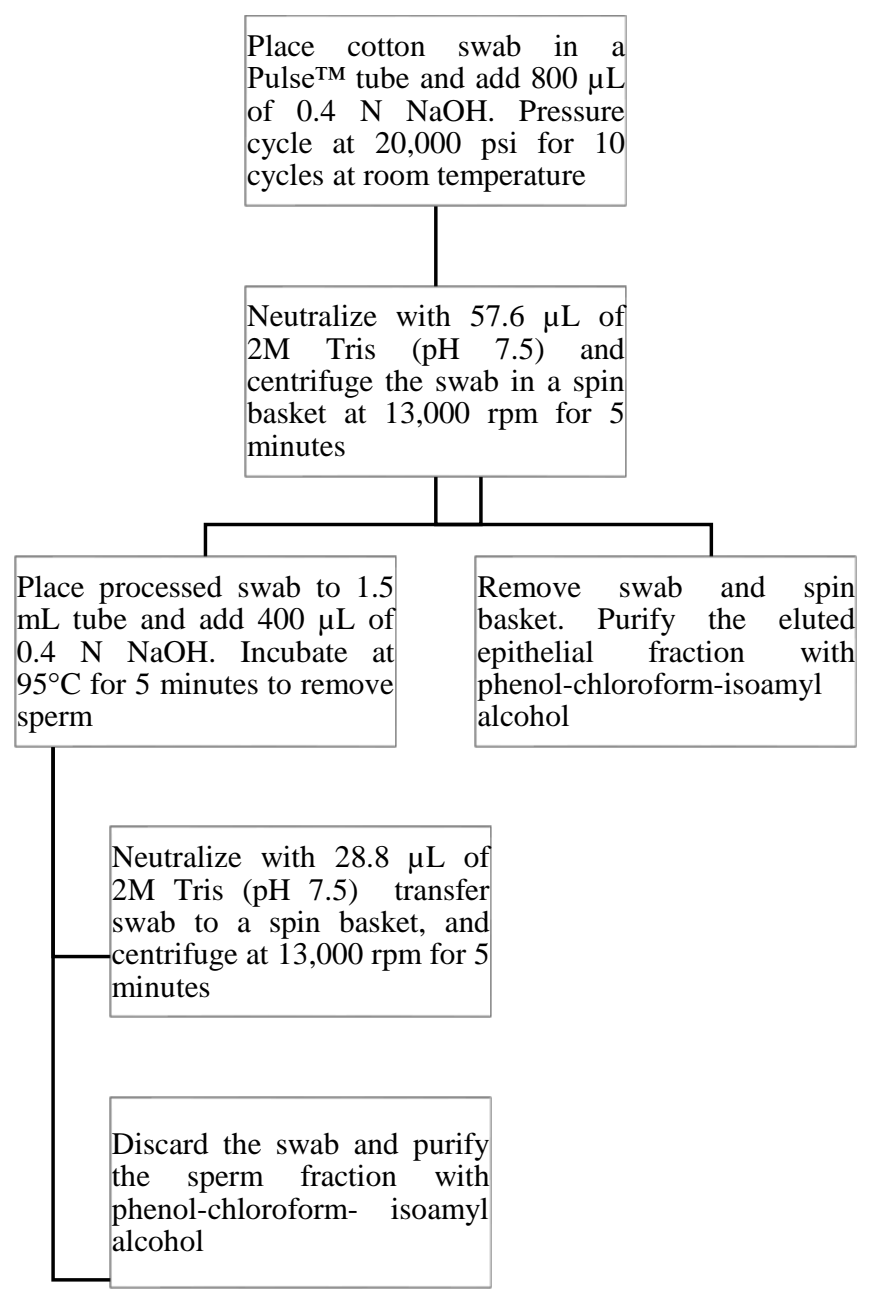

Figure 21. Flow chart depicting the developed PCT + alkaline lysis protocol. The chart details steps for obtaining separate epithelial and sperm fractions [75].

This method results in reported recovery of $104 \pm 6 \%$ for female epithelial cell DNA and $69 \pm 6 \%$ for sperm cell DNA according to experiments involving 1:1 mixtures. Complete separation can be achieved at female to male cell ratios of $1: 1$ or $2: 1$, but as the ratio increases the sample is overwhelmed with female DNA. These results are displayed in Figure 22. The separation becomes less complete and male allelic dropout is observed [75]. 


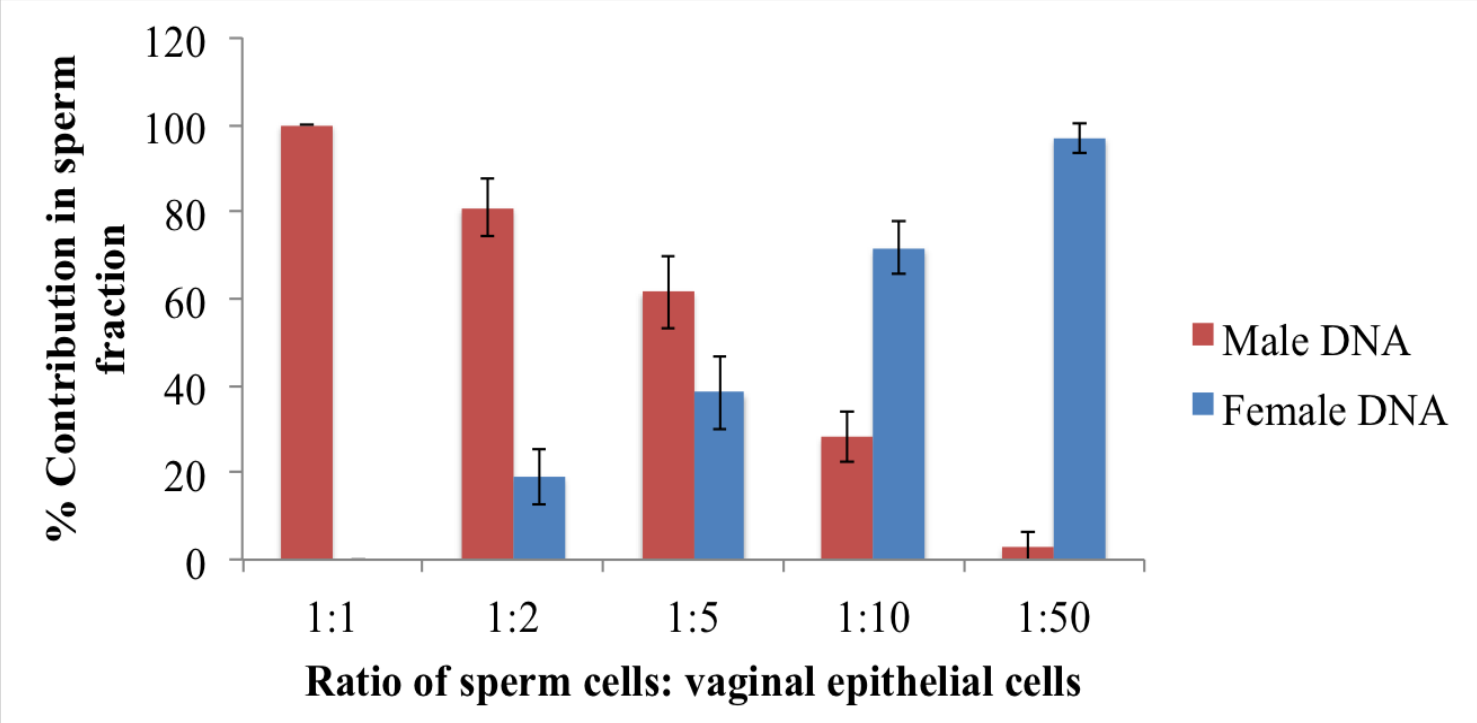

Figure 22. Percent contribution of male and female alleles in sperm fraction. Allelic peak heights from male and female contributors were divided by the total peak height at the respective locus. A total of seven loci with no shared alleles between the male and female DNA profiles were selected to calculate the percent contribution of male and female DNA [75].

Steps must be taken to optimize and improve this protocol. The desired goal is the development of a method for differential extraction that is rapid, selective, and reliable, has results with high recovery, and yields a complete male profile even with a large number of female cells in the sample. Variables during the pressure based lysis step could be optimized to achieve this objective. The concentration of $\mathrm{NaOH}$, temperature, and number of cycles can be varied for maximum recovery and removal of epithelial cell DNA from the substrate. Additional rounds of pressure cycling can also be incorporated to remove any epithelial cell DNA left behind in the swab. 


\section{B. Materials and Methods}

\section{B.1. Sample Preparation}

Epithelial cell samples were collected from healthy female volunteers via approved protocols per the institutional review board (IRB) of Florida International University. Vaginal swabs were placed in $1.5 \mathrm{~mL}$ tubes with $1 \mathrm{~mL}$ of $1 \mathrm{X}$ PBS buffer (pH 7.5) (Fisher Scientific, Fair Lawn, NJ). Samples were agitated for 1 hour on an Adams Nurator (Clay Adams, Parsipanny, NJ). Swabs were then placed in a spin basket and centrifuged at $13,000 \mathrm{rpm}$ for 10 minutes to obtain a cell pellet. The buffer was then decanted and the pellet was diluted to the desired concentration with 1X PBS buffer. Sperm cell samples were obtained from Fairfax Cryobank (Fairfax, VA). Samples were allowed to liquefy at room temperature. Aliquots were diluted to a desired concentration with the addition of $1 \mathrm{X}$ PBS buffer.

\section{B.2. Cell Count}

Cell density was determined with the use of a Neubauer-improved disposable Cchip hemocytometers (INCYTO, Fisher Scientific, Fair Lawn, NJ). Each hemocytometer contains a large grid of nine squares, with the four corner squares divided into sixteen squares each for cell counting. The injection port is filled with $10 \mu \mathrm{L}$ of sample and examined with a light microscope under 40x magnification for epithelial cells and 100x magnification for sperm cells. The number of cells in each of the four squares is counted and an average is calculated. Each of the nine squares has a surface area of $1 \mathrm{~mm}^{2}$ and a depth of $0.1 \mathrm{~mm}$ for a volume if $10^{-4} \mathrm{~cm}^{3}$. The product of the average and $10^{4}$ then

provides a cell concentration in cells/mL. Sperm cell suspensions were adjusted to a 
concentration of 250,000 cells $/ \mathrm{mL}$. Epithelial cell suspensions were prepared and adjusted to concentrations of 2,500,000 cells /mL, 1,250,000 cells/mL, and 500,000 cells/mL. The female epithelial cell to sperm cells ratio was ranged from 20:1, 50:1, 100:1, and 200:1.

\section{B.3. Pressure Cycling Technology (PCT) Sample Preparation}

Samples were prepared with the addition of $100 \mu \mathrm{L}$ of epithelial cell suspension and $10 \mu \mathrm{L}$ of sperm cell suspension on a sterile cotton swab (Puritan Medical Products Co., Guilford, ME). The swab was then left to dry at room temperature for 1 hour.

DNA extraction of the epithelial cells was accomplished through pressure cycling with the use of the Barocycler ${ }^{\circledR}$ NEP 2320 from Pressure BioSciences, Inc. (South Easton, MA). The sample swab was cut into a FT500-ND PULSETM tube (BioSciences, Inc.,South Easton, MA) specifically manufactured for lysis with the Barocycler ${ }^{\circledR}$ instrument. The addition of $800 \mu \mathrm{L}$ of $\mathrm{NaOH}$ prepared from sodium hydroxide crystals (Fisher Scientific, NJ) dissolved in molecular grade water (Fisher Scientific, NJ) completely submerged the substrate and the cap of the tube was set in place sealing the PULSE $^{\text {TM }}$ tube. The tube, which includes a moveable ram that transmits pressure to the sample, was then inserted into the hydrostatic chamber of the instrument. Concentration of $\mathrm{NaOH}$ varied from $0.4 \mathrm{~N}, 0.2 \mathrm{~N}, 0.1 \mathrm{~N}, 0.05 \mathrm{~N}$, and $0.025 \mathrm{~N}$.

Temperature during pressure cycling was adjusted from $20^{\circ} \mathrm{C}, 25^{\circ} \mathrm{C}, 32^{\circ} \mathrm{C}$, and $45^{\circ} \mathrm{C}$ by connection to an Endocal RTE-110 water circulator (Neslab Instruments, Inc., Newington). Each cycle consisted of the application of target pressure to the sample followed by ambient pressure. The time at target pressure and ambient pressure was set 
at 15 seconds each. Target pressure remained set at 20,000 psi as in the original protocol. Each round of pressure cycling was followed by transferring of the supernatant into a 2 $\mathrm{ml}$ tube, inserting of the swab into a spin basket (Kerafast, Inc., Boston, MA), and centrifuging at 13,000 rpm for 5 minutes. The collected solution contained the epithelial fraction which was ready for purification. The swab was saved and transferred to a 1.5 $\mu \mathrm{L}$ tube for further processing.

\section{B.4. Alkaline Lysis and Neutralization}

Alkaline lysis of the remaining sperm cells was performed with the addition of $400 \mu \mathrm{L}$ of $0.4 \mathrm{~N} \mathrm{NaOH}$ and incubation in a $95^{\circ} \mathrm{C}$ water bath for 5 minutes. The swab was then inserted into a spin basket and centrifuged at 13,000 rpm for 5 minutes to obtain the sperm fraction.

Neutralization was initially carried out with the application of $2 \mathrm{M}$ Tris ( $\mathrm{pH} 7.5)$ (Fisher Scientific, NJ). This was found to be ineffective and lead to low DNA concentrations with the use of the EZ1 DNA Investigator Kit and the EZ1 Advanced (Qiagen, Valencia, CA) due to the $\mathrm{pH}$ dependence of the procedure. This step was replaced with the addition of $160 \mu \mathrm{L}$ of $1 \mathrm{M} \mathrm{HCl}$ (Fisher Scientific, NJ) to the sperm fraction.

\section{B.5. DNA Purification}

Sperm fraction lysates were purified with EZ1 DNA Investigator Kit and the EZ1 Advanced (Qiagen, Inc., Valencia, CA). This method involves DNA binding to a silica surface on magnetic particles through the addition of chaotropic salt. The DNA is 
then washed and eluted yielding a pure DNA sample. The instrument can purify up to 6 samples in a single run and handle sample volumes of $200-500 \mu \mathrm{L}$. The large-volume protocol was selected for purification of the sperm lysates which were eluted in $40 \mu \mathrm{L}$ of 1X Tris-EDTA buffer (TE) buffer.

The epithelial fractions were purified by the addition of an equal volume of phenol-chloroform-isoamyl alcohol (25:24:1) (Sigma-Aldrich, St.Louis, MO) and precipitated with $3 \mathrm{M}$ sodium acetate and $100 \%$ ethanol. The resulting pellet was washed with $70 \%$ ethanol and air dried. It was finally resuspended in $1 \mathrm{X}$ Tris-EDTA buffer (pH 8.0) (Fisher Scientific, NJ) and incubated at $56^{\circ} \mathrm{C}$ for 15 minutes.

\section{B.6. DNA Quantitation}

Amplification and quantitation of DNA extracted from the samples was accomplished using the Plexor ${ }^{\circledR}$ HY system (Promega Corp., Madison, WI) on the RotorGene 6000 (Corbett, Australia). Quantification of both autosomal and Y-chromosomal DNA was necessary to determine the selectivity of the procedure through calculation of a Y/Autosomal DNA ratio and to resolve the optimum amount of sample required for PCR amplification. The standard curve was constructed with diluted male genomic DNA standard included by the manufacturer. Reactions were prepared per manufacturer's protocols using $10 \mu \mathrm{L}$ Plexor ${ }^{\circledR}$ HY $2 X$ Master Mix, $7 \mu \mathrm{L}$ amplification-grade water, $1 \mu \mathrm{L}$ Plexor $^{\circledR}$ HY 20X Primer/IPC Mix and $2 \mu$ LNA for a total of $20 \mu \mathrm{L}$ per reaction.

Total DNA concentration and male DNA concentration was determined though autosomal DNA and Y-chromosomal DNA quantitation results respectively. The female 
DNA concentration in the epithelial fraction was determined by subtraction of quantified Y-chromosomal DNA from quantified autosomal DNA.

\section{B.7. PCR Amplification}

The quality of recovered DNA was assessed by performing STR analysis with the PowerPlex ${ }^{\circledR} 16$ HS system (Promega Corp., Madison, WI) according to manufacturer's protocols. The system contains primers for sixteen loci including the Amelogenin sex determination marker. Fluorescein-labeled primers are used for the detection of Penta E, D18S51, D21S11, TH01 and D3S1358 loci; 6-carboxy-4',5'dichloro-2', $7^{\prime}$-dimethoxy-fluorescein (JOE) labeled primers were used for the detection of Penta D, CSF1PO, D16S539, D7S820, D13S317 and D5S818 loci; and carboxytetramethylrhodamine (TMR) labeled primers for FGA, TPOX, D8S1179, vWA and Amelogenin.

\section{B.8. STR Analysis}

Samples were amplified using ABI GeneAmp® 9700 thermal cyclers (Applied Biosystems, Foster city, CA). Amplification products were then processed using ABI PRISM $^{\mathrm{TM}} 310$ genetic analyzer (Applied Biosystems, Foster City, CA) and GeneMapper $^{\circledR}$ v.4.0 (Applied Biosystems, Foster City, CA) to obtain genotype profiles. Samples were prepared for STR analysis with $1 \mu \mathrm{L}$ of sample to a mixture of $9.5 \mu \mathrm{L}$ of Hi-Di ${ }^{\mathrm{TM}}$ formamide (Applied Biosystems, Foster city, CA) and $0.5 \mu \mathrm{L}$ of Internal Lane Standard 600 (Promega Corp., Madison, WI). 


\section{Results and Discussion}

\section{C.1. NaOH Concentration Optimization}

Previous work by Nori et al. determined the optimal concentration of $\mathrm{NaOH}$ for the lysis of sperm cells was $0.4 \mathrm{~N}$ through experiments investigating the effects of varying concentrations of $\mathrm{NaOH}(0.2-1 \mathrm{~N})$, temperature $\left(75^{\circ} \mathrm{C}-95^{\circ} \mathrm{C}\right)$, and incubation time $(2$ or 5 minutes). It was discovered that $0.4 \mathrm{~N} \mathrm{NaOH}$ at $95^{\circ} \mathrm{C}$ for 5 minutes resulted in high recovery of sperm cell DNA, $99.6 \pm 1.0 \%$, and low recovery of female epithelial cell DNA, $41 \pm 2 \%$. This concentration was then selected for further optimization of the differential extraction protocol during both cell lysis steps. This concentration of $\mathrm{NaOH}$ may not be suitable for the PCT step since the goal is female epithelial cell lysis with minimal disruption of sperm cells. It was noted that the best recovery of female DNA occurred with the use of $0.2 \mathrm{~N} \mathrm{NaOH}, 78 \pm 21 \%$ [75]. Considering this data, the PCT step was tested at both concentrations and results were compared with the sperm cell lysis step remaining unaltered. As $0.2 \mathrm{~N}$ was the lowest concentration examined in previous studies, experiments were also performed at $0.1 \mathrm{~N}, 0.05 \mathrm{~N}$, and $0.025 \mathrm{~N}$. The optimal concentration was then determined and selected for further experiments.

Initial experiments were performed to determine the effect of $\mathrm{NaOH}$ on the recovery and selectivity of analysis. The results can be observed in Figure 23. The selectivity of the protocol was determined through calculation of male DNA and female DNA contribution to generated STR profiles of the sperm fraction obtained from each sample. A total of seven loci were selected that share no alleles in common between the profiles of male and female contributors. Relative Fluorescence Units (RFU) of peaks from 
male and female contributors were divided by total RFU at each locus x100 for calculation of percentage.

The highest selectivity using a 20:1 female epithelial cell to sperm cell sample was achieved with the use of $0.05 \mathrm{~N} \mathrm{NaOH}$ with a male DNA contribution of $71 \pm 1.6 \%$. Similar results occurred at $0.025 \mathrm{~N}$ with $65 \pm 3.8 \%$ and intermediate results were obtained at $0.1 \mathrm{~N}$ with $58 \pm 0.98 \%$. The selectivity at these concentrations are superior to that achieved at $0.2 \mathrm{~N}$ with a male DNA contribution of $43 \pm 2.6 \%$. An even lower male DNA contribution resulted from using the original concentration of $0.4 \mathrm{~N}$ with $18 \pm 0.92 \%$.

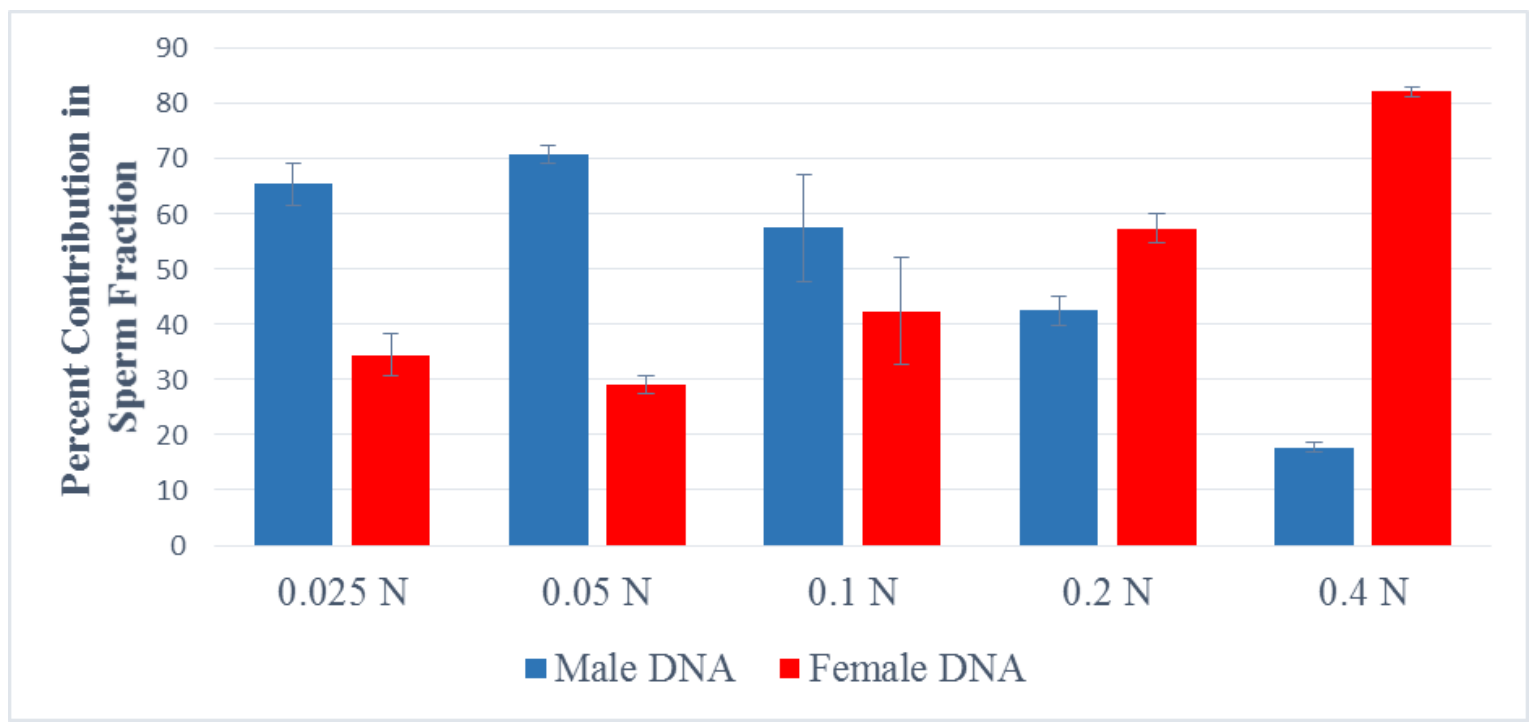

Figure 23. The effect of $\mathrm{NaOH}$ concentration on selectivity on 20:1 F:M samples. The highest male DNA contribution percentage was obtained at $0.05 \mathrm{~N}$ and the lowest at the original $0.4 \mathrm{~N}$ concentration. Percentage determined by male or female peak height divided by total peak height at 7 loci where no alleles are shared between the male or female contributor, $x 100$. $(n=3 \pm$ standard error $)$

Investigation of the epithelial fraction can shed some light on these results. The female DNA recovered in the epithelial fraction is displayed in Figure 24. The highest concentration of female DNA was recovered with $0.1 \mathrm{~N} \mathrm{NaOH}, 7.02 \pm 2.1 \mathrm{ng} / \mu \mathrm{L} .0 .05 \mathrm{~N}$ 
and $0.2 \mathrm{~N}$ showed similar results with $6.86 \pm 1.9 \mathrm{ng} / \mu \mathrm{L}$ and $6.87 \pm 0.36 \mathrm{ng} / \mu \mathrm{L}$ respectively. At higher and lower concentrations, $0.025 \mathrm{~N}$ and $0.4 \mathrm{~N}$, recovery suffered, with only $5.79 \pm 1.6 \mathrm{ng} / \mu \mathrm{L}$ and $5.30 \pm 1.5 \mathrm{ng} / \mu \mathrm{L}$.

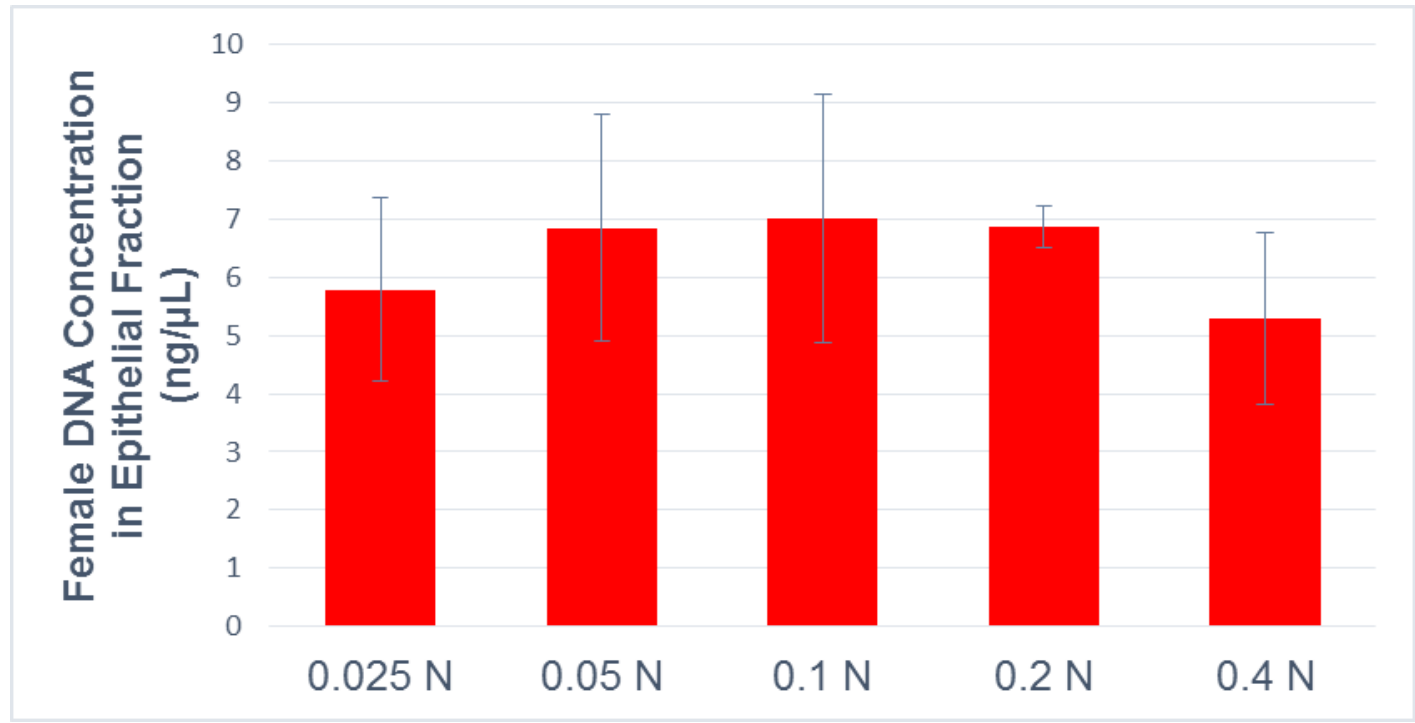

Figure 24. The effect of $\mathrm{NaOH}$ concentration on female DNA recovery in the epithelial fraction obtained after the PCT step. The highest recovery occurred between $0.05 \mathrm{~N}$ and $0.2 \mathrm{~N}$. Higher and lower concentrations, $0.025 \mathrm{~N}$ and $0.4 \mathrm{~N}$, decreased recovery. $(\mathrm{n}=3 \pm$ standard error)

$\mathrm{NaOH}$ concentration affects male DNA loss in the epithelial fraction as well. Figure 25 depicts the concentration of male DNA found in the epithelial fraction at each concentration of $\mathrm{NaOH}$. This DNA loss increases as $\mathrm{NaOH}$ concentration increases. It can be concluded that harsher alkaline conditions disrupt the sperm cells during the PCT step and decrease selectivity. 


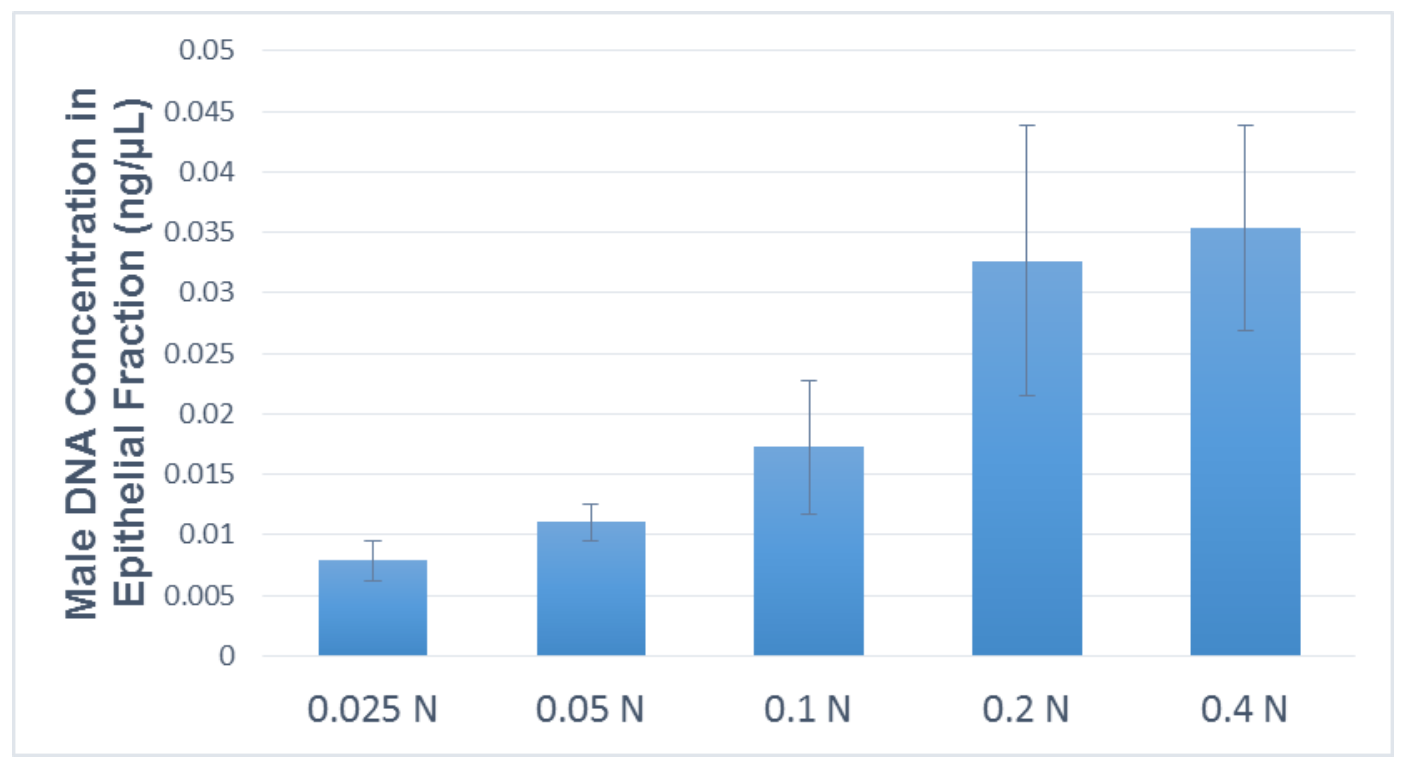

Figure 25. The effect of $\mathrm{NaOH}$ concentration on male DNA loss during the PCT step. As $\mathrm{NaOH}$ concentration increased, more male DNA was lost in the epithelial fraction during PCT. It is likely the result of the disruption of sperm cells by harsh alkaline conditions. $(n=3$ \pm standard error)

The $\mathrm{NaOH}$ concentration selected for further experiments was $0.05 \mathrm{~N}$. Increased female DNA recovery and decreased male DNA loss during the PCT step strike a balance that leads to greater selectivity and higher male DNA contributions in STR profiles obtained from the sperm fraction.

Figures 26 and 27 display the yellow panel of an STR profile generated with PowerPlex ${ }^{\circledR} 16 \mathrm{HS}$ of the male and female contributor respectively. Figure 28 displays an STR profile of the sperm fraction of a sample extracted with the protocol modified with $0.05 \mathrm{~N} \mathrm{NaOH}$ used during the PCT step. The profile is majority male with a male DNA contribution of 68\%. For comparison, Figure 29 displays and STR profile from the sperm fraction of a mixture processed with the original two-step protocol using $0.4 \mathrm{~N}$ $\mathrm{NaOH}$ for the PCT step. The profile is majority female with a male contribution of $16 \%$. 


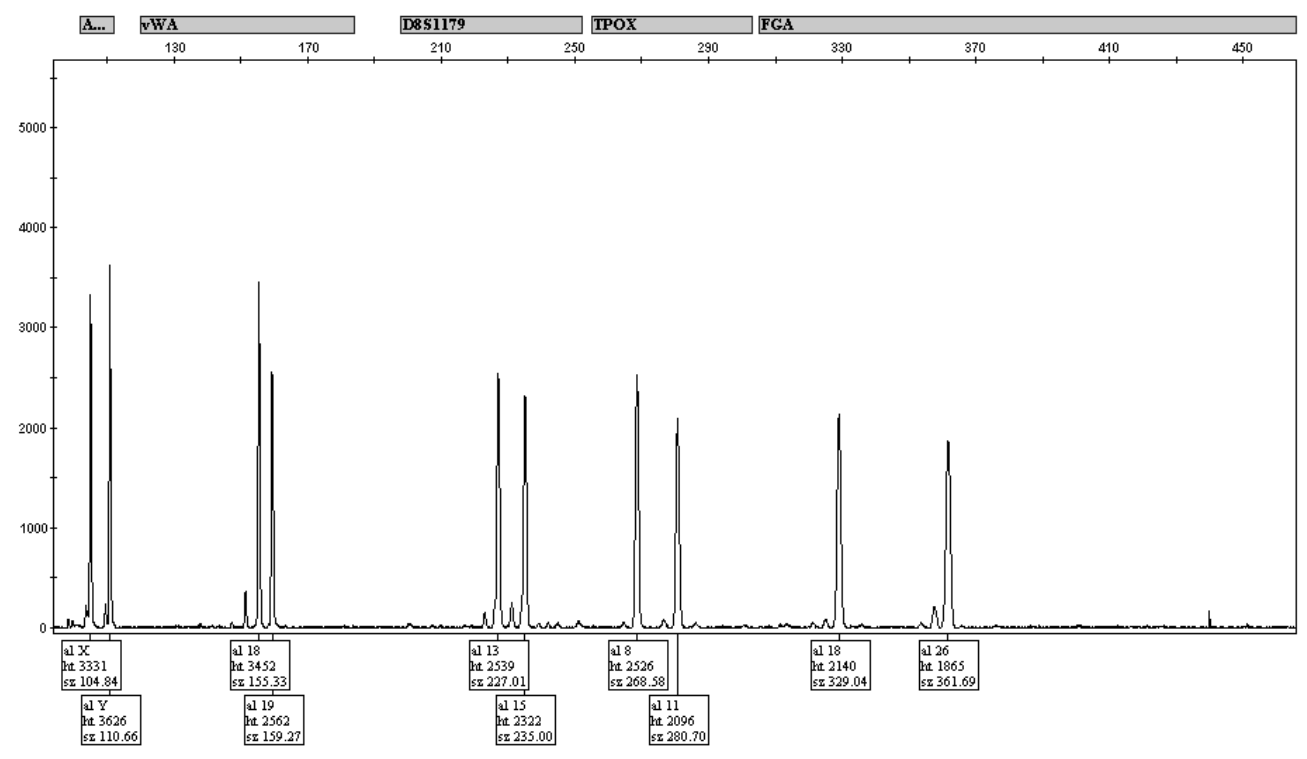

Figure 26. Profile of the male contributor. Panel represents loci labeled with 5-TAMRA (5Carboxytetramethylrhodamine); Amelogenin, D8S1179, TPOX, and FGA.

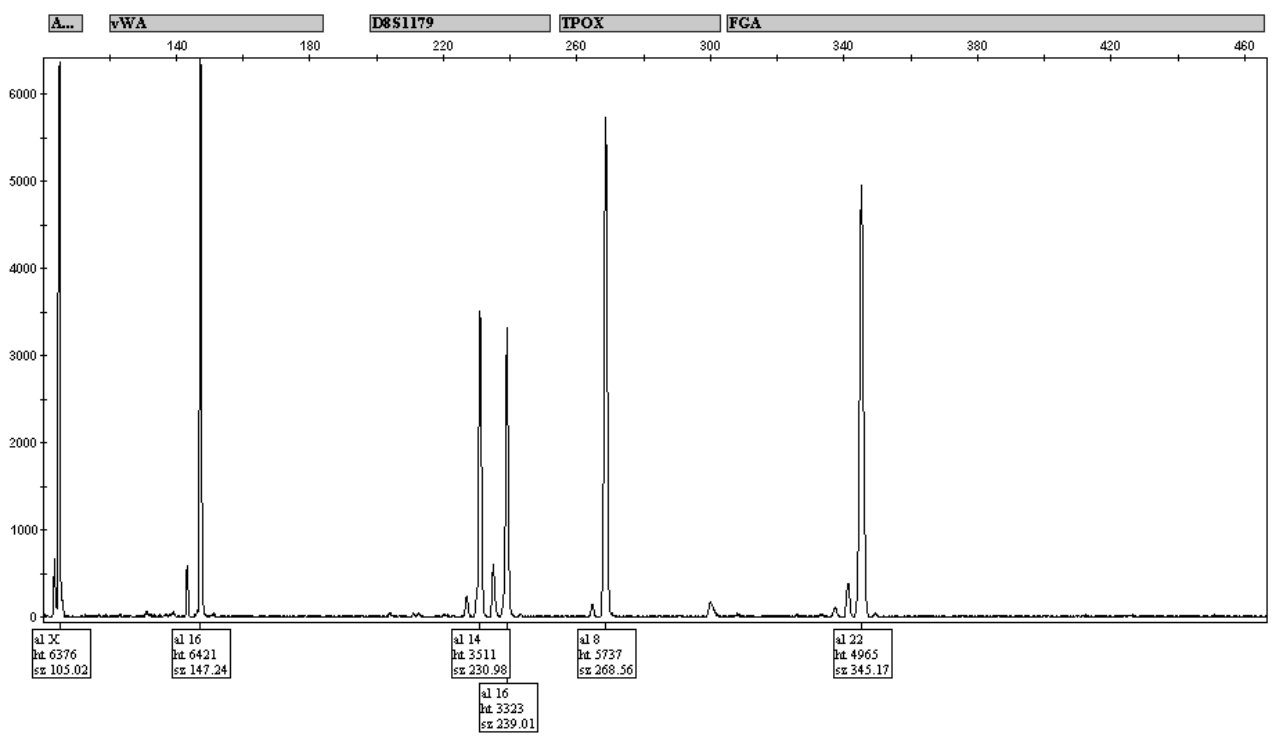

Figure 27. Profile of the female contributor. Panel represents loci labeled with 5-TAMRA (5-Carboxytetramethylrhodamine); Amelogenin, D8S1179, TPOX, and FGA. 


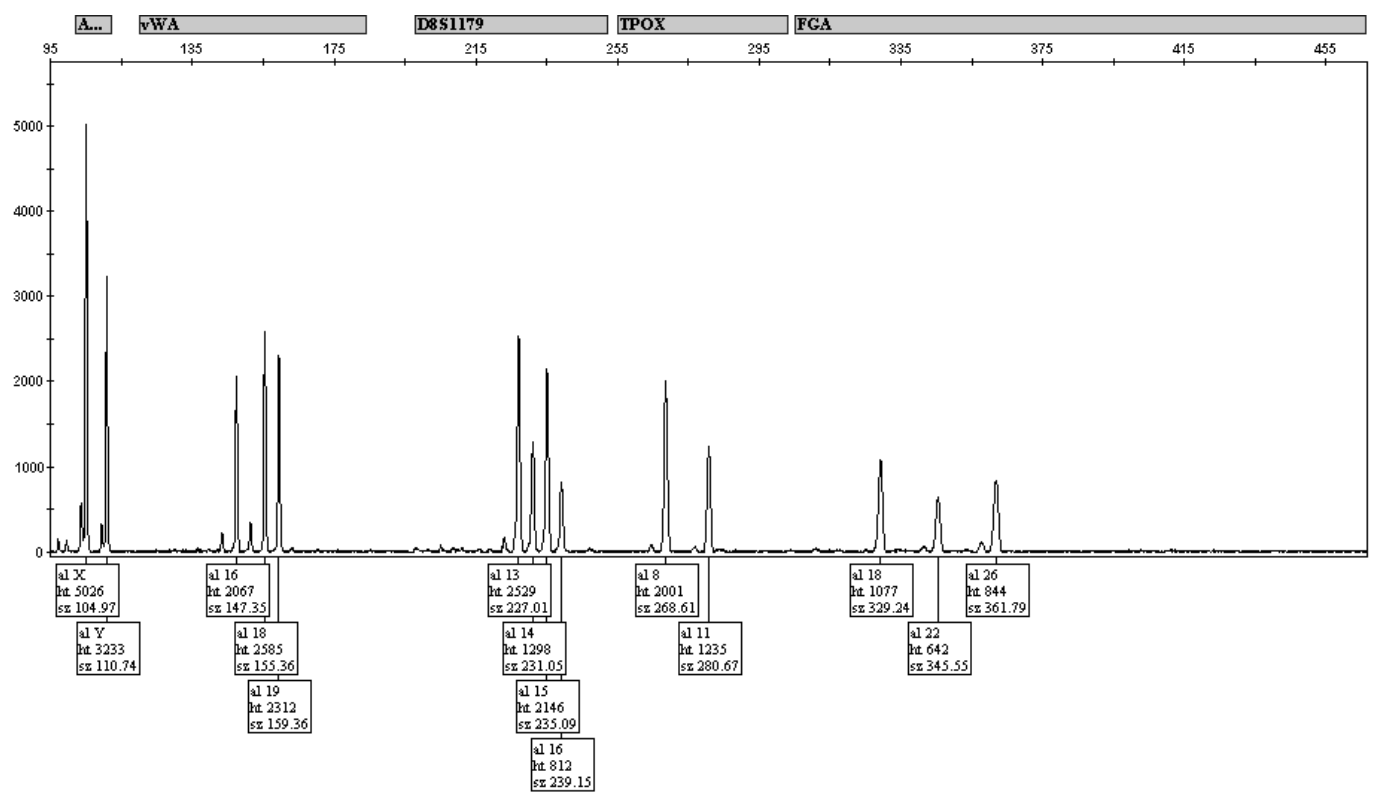

Figure 28. Sperm fraction profile of a 20:1 F:M sample extracted with PCT + alkaline lysis protocol modified with $0.05 \mathrm{~N} \mathrm{NaOH}$ for the PCT step. A slight majority male profile is observed with a male DNA contribution of $68 \%$. Panel represents loci labeled with 5TAMRA (5-Carboxytetramethylrhodamine); Amelogenin, D8S1179, TPOX, and FGA.

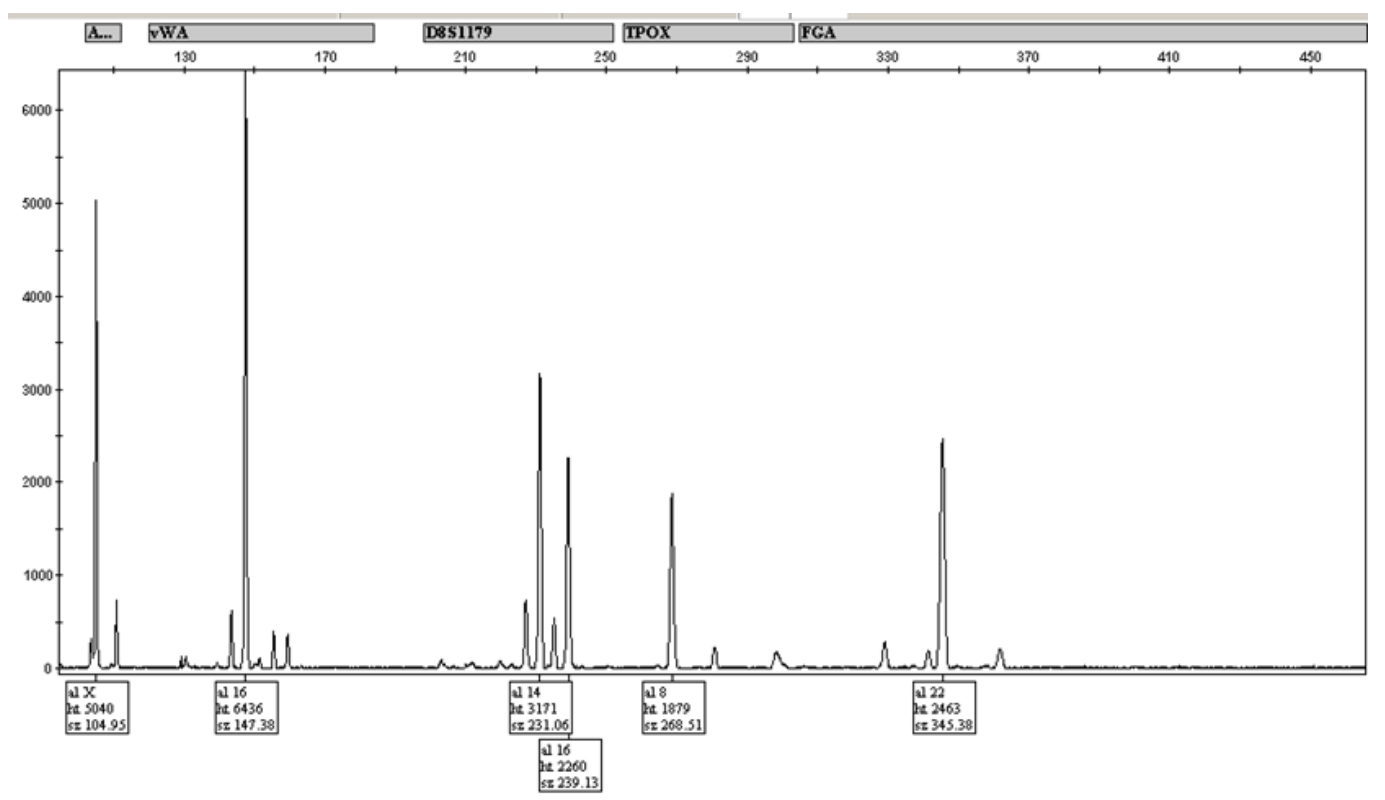

Figure 29. Sperm fraction profile of a 20:1 F:M sample extracted with original PCT + alkaline lysis protocol with $0.4 \mathrm{~N} \mathrm{NaOH}$ for the PCT step. The percent contribution of male DNA in the profile was only $16 \%$. Panel represents loci labeled with 5-TAMRA (5Carboxytetramethylrhodamine); Amelogenin, D8S1179, TPOX, and FGA. 


\section{C.2. Temperature Optimization}

Additional experiments were conducted to further optimize the temperatures used in the lysis step. The Barocycler ${ }^{\circledR}$ NEP 2320 can be run at temperatures ranging from $4-60^{\circ}$ C. previous work was performed at room temperature. Higher temperatures may be used during the PCT step to facilitate recovery and removal of epithelial cell DNA, but care must be taken not to disturb the sperm cells during this step. The application of lower temperatures may also improve results by impeding the removal of male DNA. Experiments were performed at the optimal $\mathrm{NaOH}$ concentration at $45^{\circ} \mathrm{C}$, $32^{\circ} \mathrm{C}$, and $20^{\circ} \mathrm{C}$. Results were then compared to experiments run at room temperature.

The effects of temperature during the PCT step with $0.05 \mathrm{~N} \mathrm{NaOH}$ is displayed in Figure 30. The initial room temperature experiments were determined to result in the highest male DNA contribution percentage. Results at $20^{\circ} \mathrm{C}$ were found to be similar, with a male DNA contribution of $66 \pm 4.2 \%$. Selectivity appeared to decrease with increasing temperature, with $58 \pm 4.5 \%$ male DNA contributions at $32^{\circ} \mathrm{C}$ and $47 \pm 2.5 \%$ at $45^{\circ} \mathrm{C}$. 


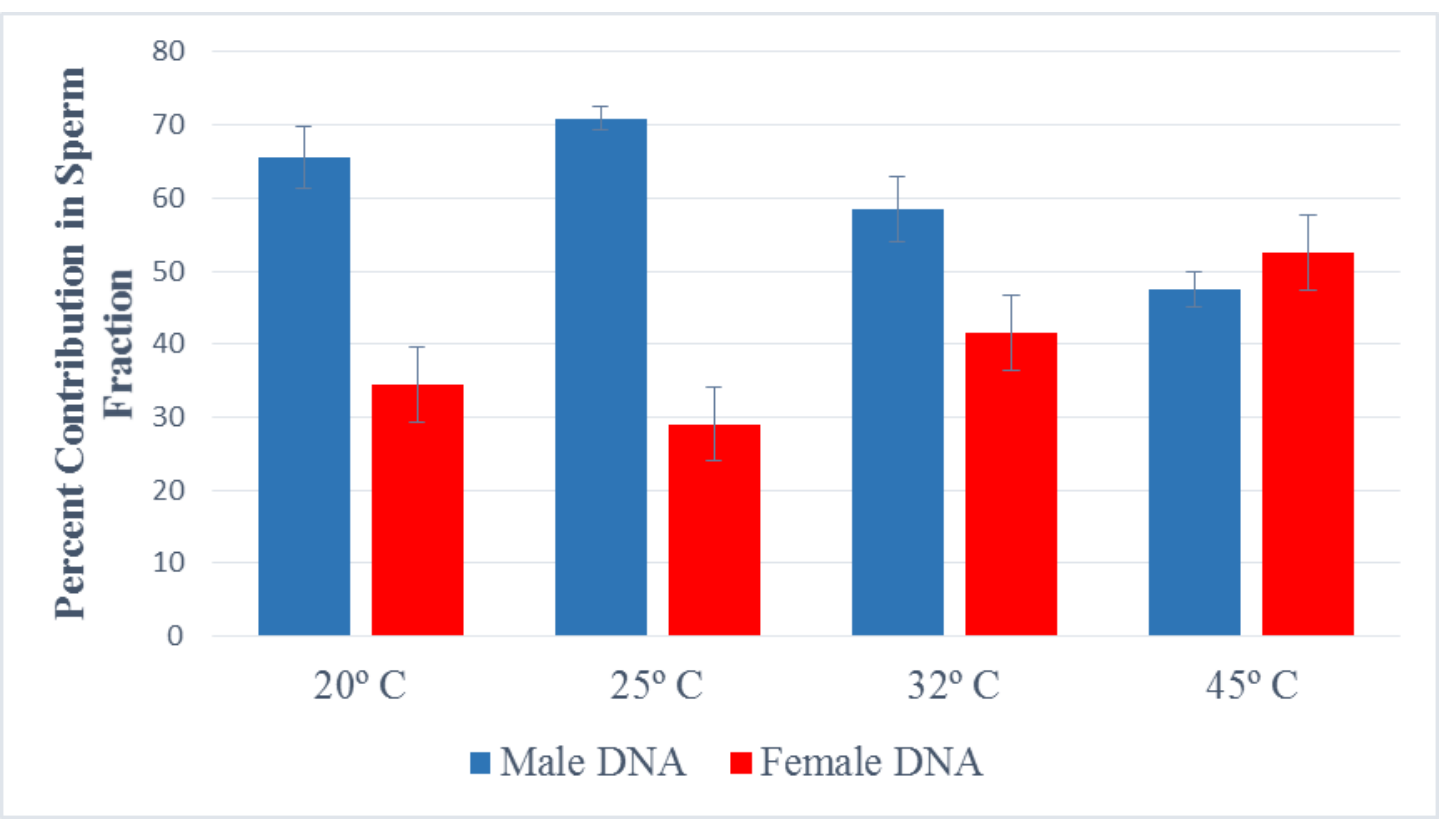

Figure 30. The effect of temperature during the PCT step on selectivity with 20:1 F:M samples. $25^{\circ} \mathrm{C}$ was the temperature that resulted in the highest male DNA contribution. Percentage determined by male or female peak height divided by total peak height at 7 loci where no alleles are shared between the male or female contributor, $x 100$. ( $(n=3 \pm$ standard error)

Figure 31 depicts the female autosomal DNA recovered in the epithelial fraction at each temperature. Aside from a $1.4 \%$ decrease found as the temperature increases for 25 to $32^{\circ} \mathrm{C}$, an increase in total recovery is observed with increasing temperature; $8.9 \%$ from 20 to $25^{\circ} \mathrm{C}$, and $8.0 \%$ from 32 to $45^{\circ} \mathrm{C}$. 


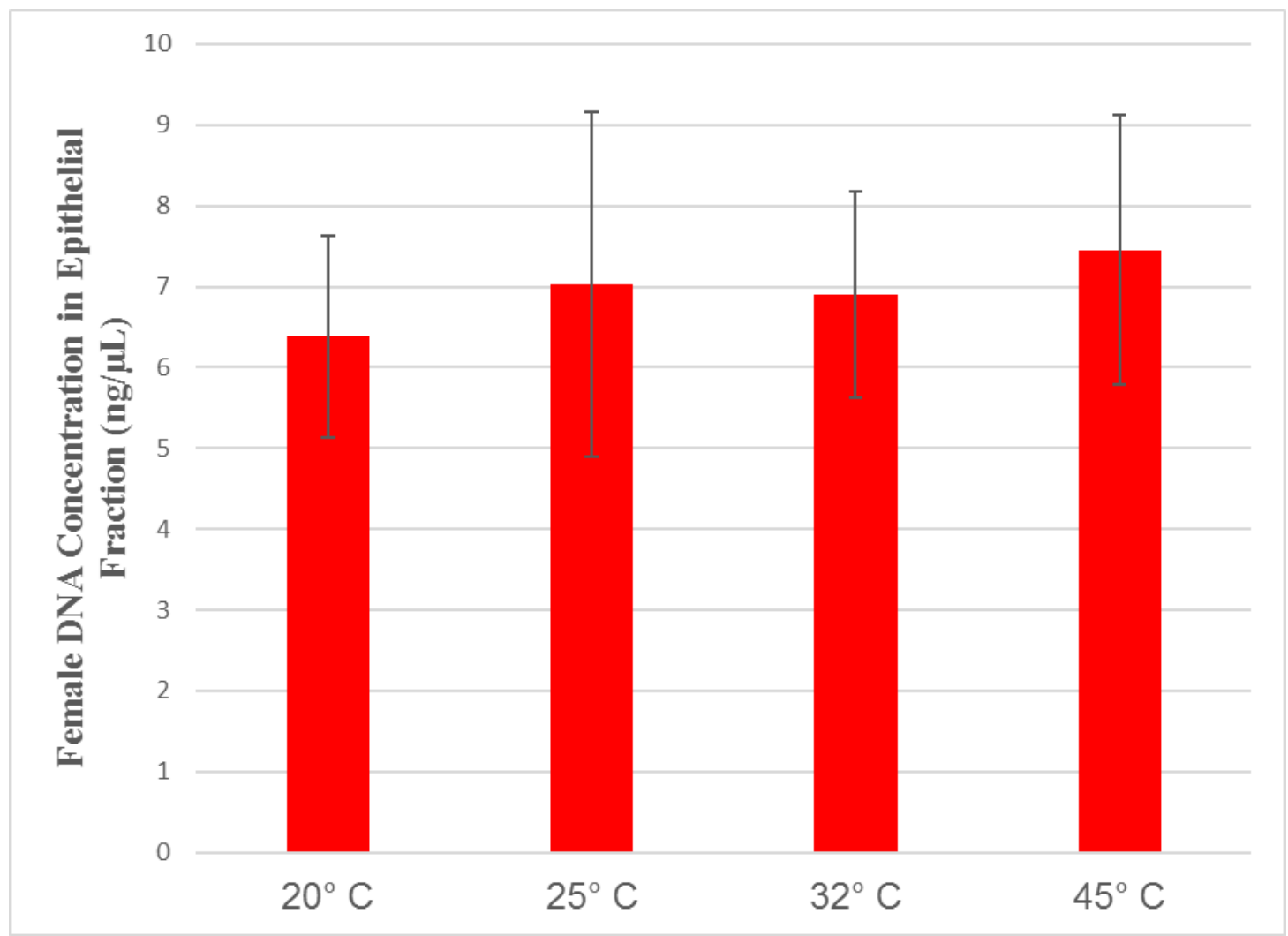

Figure 31. The effect of temperature on female autosomal DNA recovery in the epithelial fraction obtained after the PCT step. There is a slight trend for increasing recovery at higher temperature. $(n=3 \pm$ standard error)

Unfortunately, as is depicted in Figure 32, part of this increase is due to a loss of male DNA as the sperm cells are disrupted by the increasing temperature. There is a clear increase in male DNA loss at $32^{\circ} \mathrm{C}$ with $0.030 \pm 0.0051 \mathrm{ng} / \mu \mathrm{L}$ and $45^{\circ} \mathrm{C}$ with $0.046 \pm 0.0087 \mathrm{ng} / \mu \mathrm{L}$. A smaller increase in male DNA loss is noted at $20^{\circ} \mathrm{C}$ compared to $25^{\circ} \mathrm{C}$ with $0.015 \pm 0.0012 \mathrm{ng} / \mu \mathrm{L}$ and $0.011 \pm 0.0015 \mathrm{ng} / \mu \mathrm{L}$ respectively. As no improvement was detected at higher or lower temperatures, further experiments were maintained at $25^{\circ} \mathrm{C}$. 


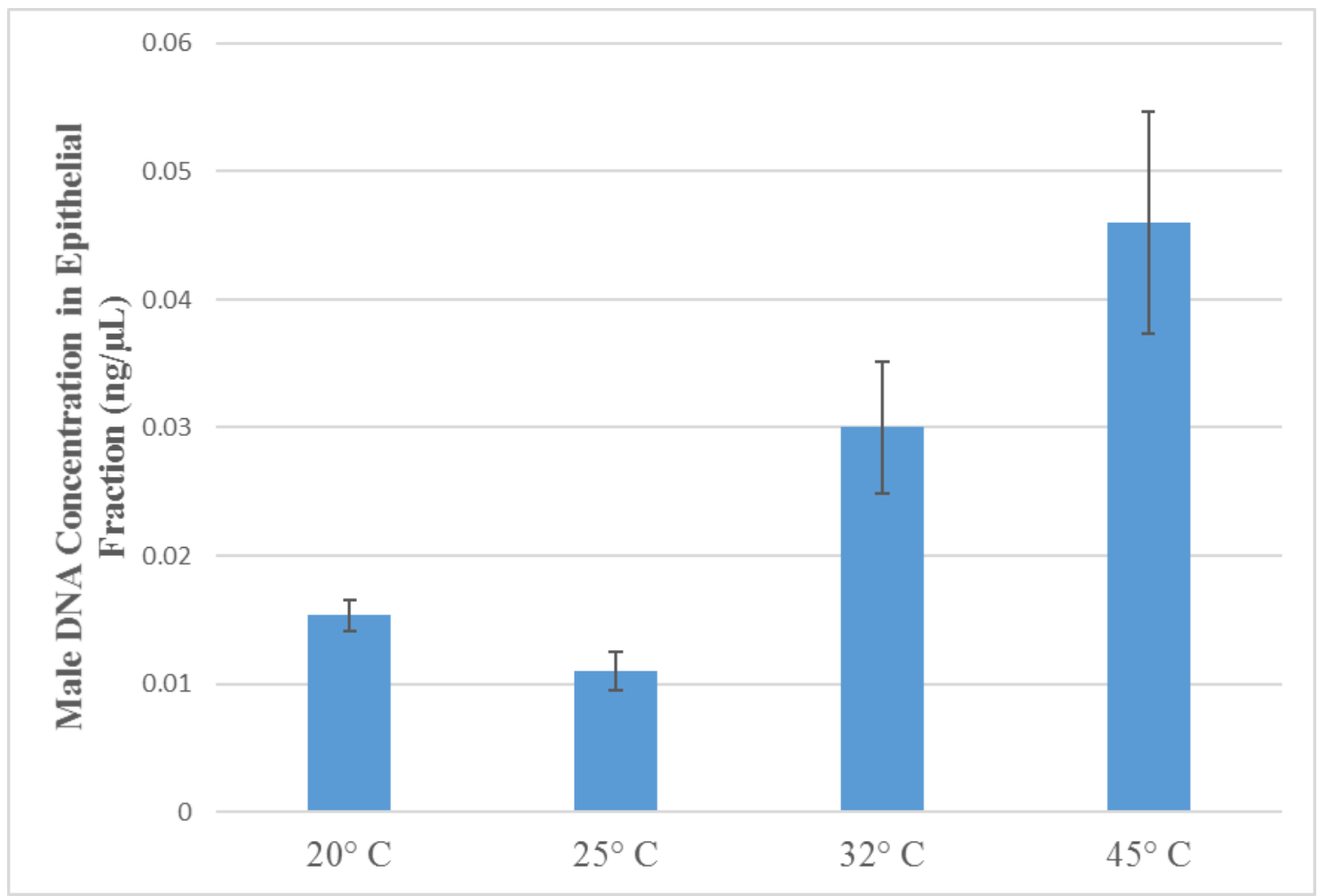

Figure 32. The effect of temperature on male DNA loss during the PCT step. At increased temperatures, more male DNA was lost in the epithelial during PCT is likely the result of the disturbance of sperm cells. $(n=3 \pm$ standard error)

\section{C.3. Extra Pressure Cycling Steps}

In order to improve recovery of female epithelial cells from the swab, the effect of a second PCT step was examined for the purpose of lysing cells and removing and removing DNA left over after the initial step. Following optimization of $\mathrm{NaOH}$ concentration and temperature, experiments were performed with the addition of an extra round of pressure cycling using 10 cycles for each round.

An extra round of pressure cycling was investigated as a way to remove epithelial cell DNA remaining in the swab. At 20:1, the selectivity of the protocol was calculated with only one round of pressure cycling (1X PCT) and with two rounds (2X PCT). The 
results are depicted in Figure 33. There is an increase in male DNA contribution with $88 \pm 0.77 \%$ compared to $71 \pm 1.6 \%$ from previous experiments. The generated STR profile is depicted in Figure 34.

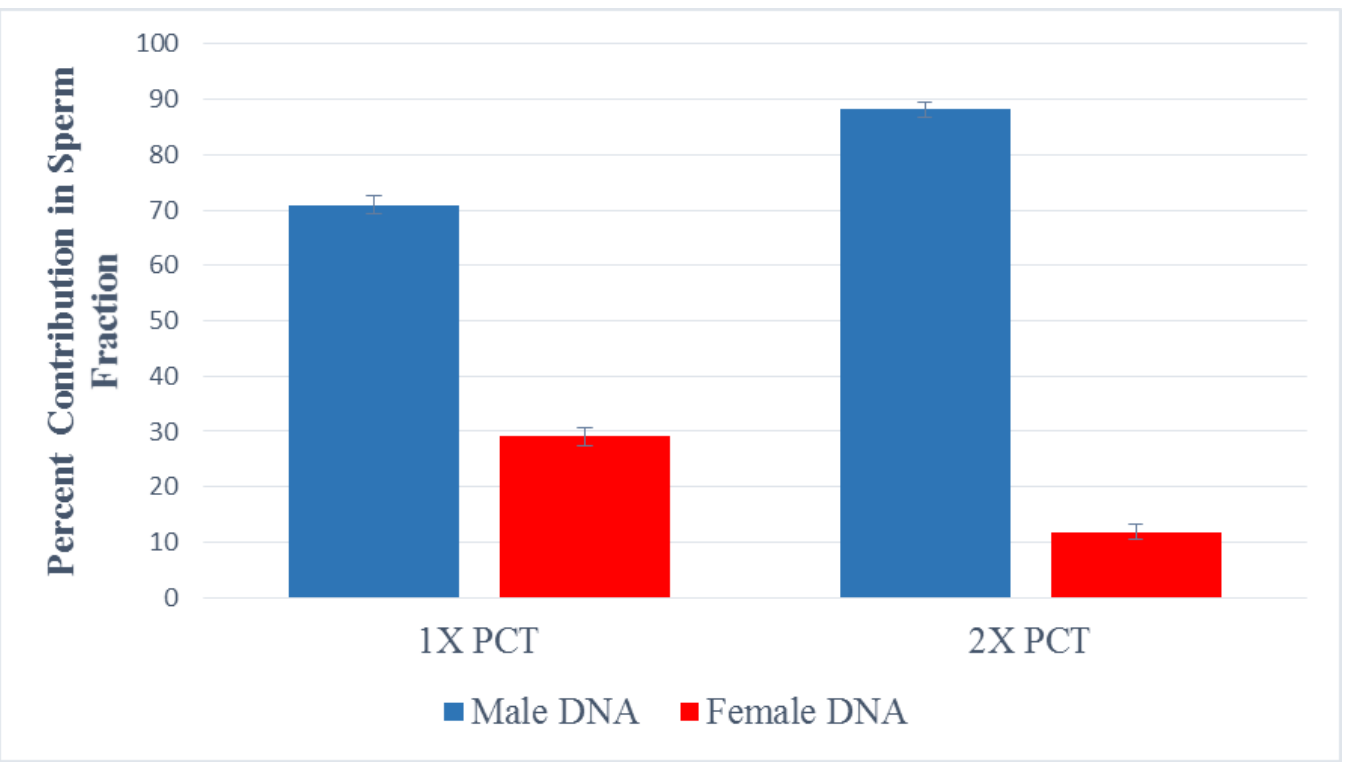

Figure 33. The effect of an additional PCT step on selectivity with 20:1 F:M samples. An increase in male DNA contribution resulted through lysis and recovery of residual epithelial cell DNA left behind in the swab. Percentage determined by male or female peak height divided by total peak height at 7 loci where no alleles are shared between the male or female contributor, $x 100(n=3 \pm$ standard error) 


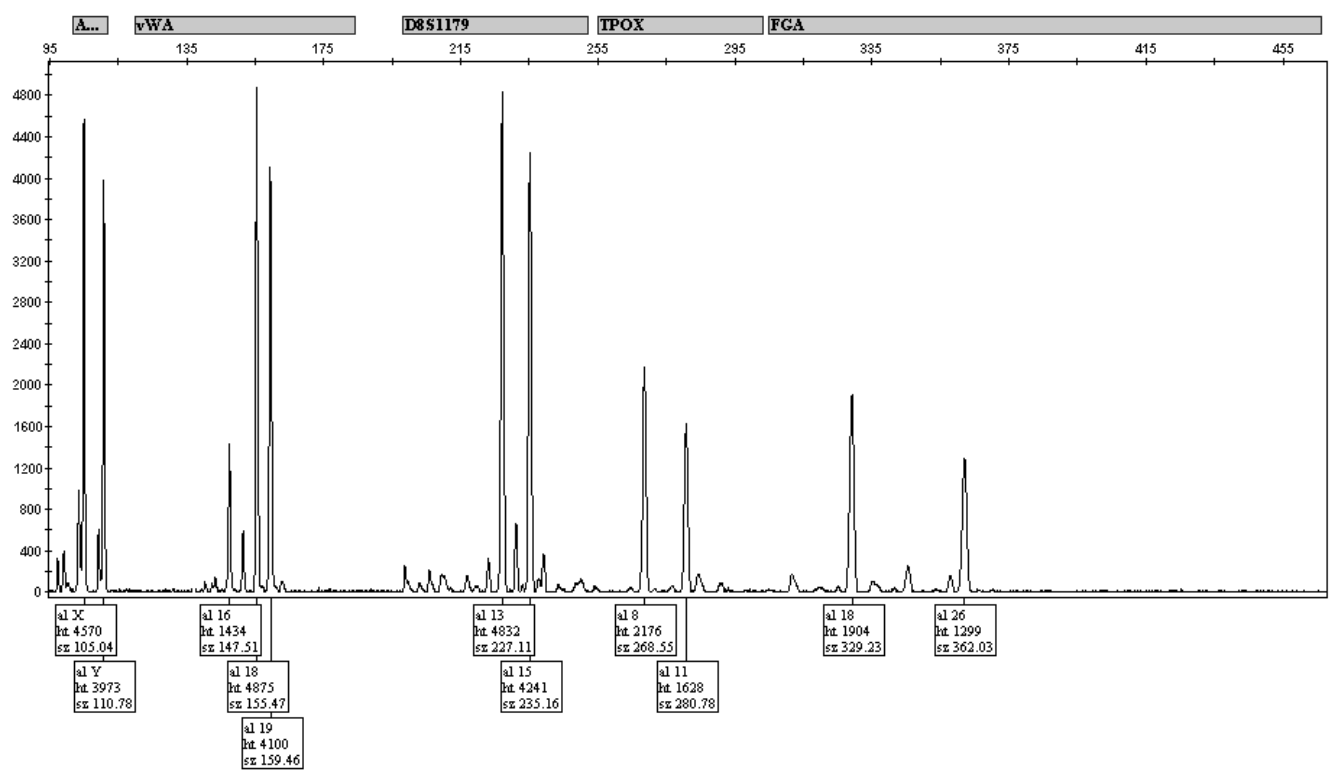

Figure 34. Sperm fraction profile of a 20:1 F:M sample extracted with PCT + alkaline lysis protocol modified with $0.05 \mathrm{~N} \mathrm{NaOH}$ for the PCT step and an additional PCT step. A percent male DNA contribution of $87 \%$ is observed in the profile. Panel represents loci labeled with 5-TAMRA (5-Carboxytetramethylrhodamine); Amelogenin, D8S1179, TPOX, and FGA.

Experiments were then performed at higher female to male cell ratios for the determination of the effects of increasing levels of female epithelial cells in the sample. The results in Figure 35 indicate a predictable decrease in selectivity. At 50:1 and 100:1, slight majority male profiles are still observed with male contributions of $67 \pm 2.0 \%$ and $59 \pm 6.0 \%$ respectively. At 200:1 a majority female profile with a male contribution of $43 \pm 13 \%$ is observed presumably due to excessive amounts of female DNA left behind in the swab. 


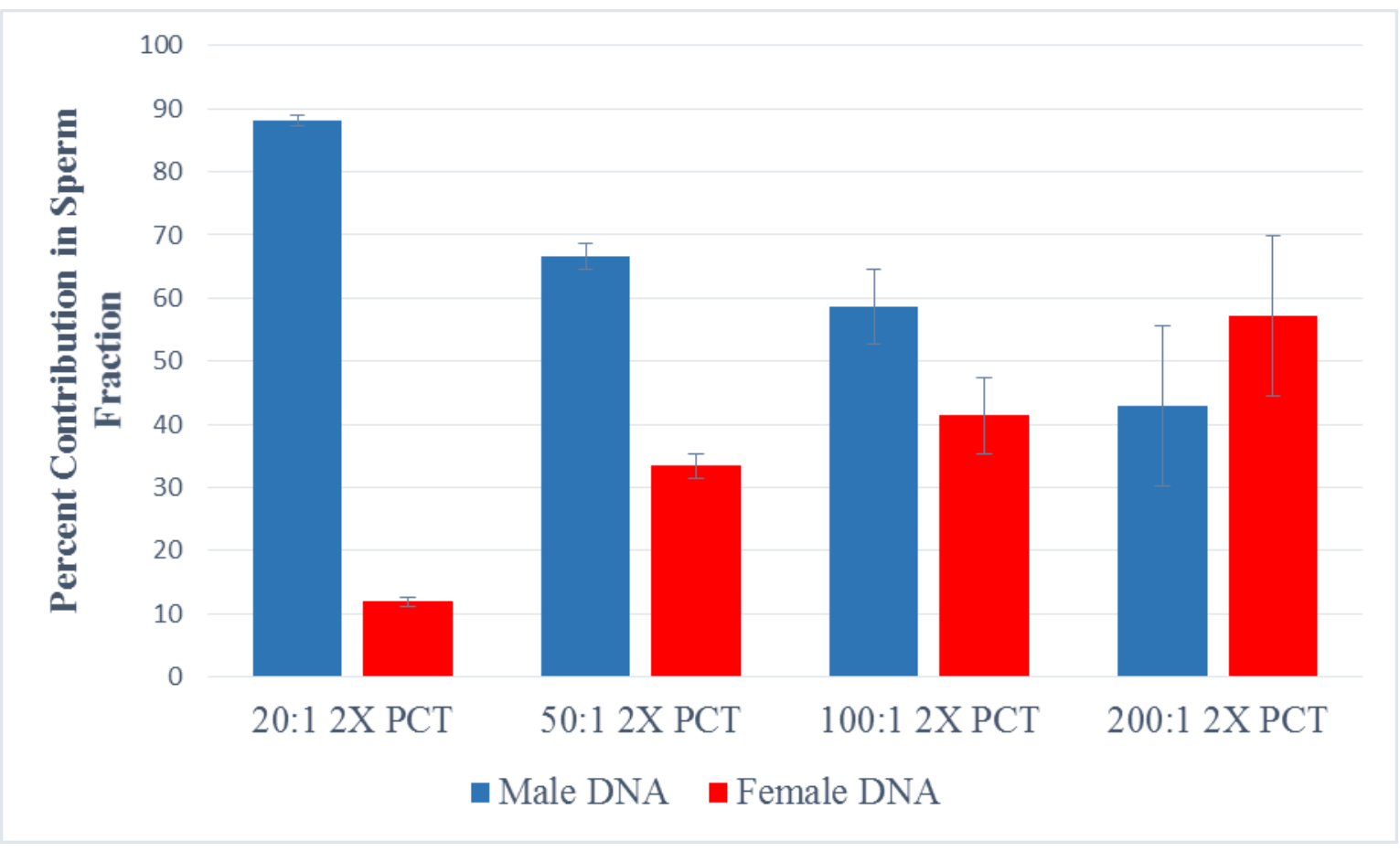

Figure 35. The effect of an additional PCT step on selectivity with 20:1, 50:1, 100:1, and 200:1 F:M samples. Predictably, selectivity decreased with increasing amounts of epithelial cells. Majority male profiles were obtained at 50:1 and 100:1. Percentage determined by male or female peak height divided by total peak height at 7 loci where no alleles are shared between the male or female contributor, $\mathbf{x 1 0 0 .}$

\section{Concluding Remarks}

Decreasing concentrations of $\mathrm{NaOH}$ clearly led to great improvement in selectivity due to decreased loss of male DNA caused by sperm cell disruption. At 0.05 $\mathrm{N}$, this effect is combined with high epithelial cell DNA recovery to yield high selectivity. An increase in temperature likely led to disturbance of sperm cells and male DNA loss. Decreasing the temperature to $20^{\circ} \mathrm{C}$ caused no improvement. Room temperature appears to be the optimal choice for future experiments. A second round of pressure cycling led to improved selectivity with a nearly clear male profile at 20:1, majority male profiles at both 50:1 and 100:1. This extra step will be incorporated into the modified protocol. Extra steps should be taken to improve the selectivity with challenging samples. 


\section{CHAPTER VII. DEVELOPMENTAL VALIDATION}

\section{A. Introduction}

The reduction of the backlog of sexual assault evidence is an important and prominent aim in current forensic research. New methods to address this issue must be proven to be reproducible, robust and effective. To demonstrate applicability for purpose, the Scientific Working Group on DNA Analysis Methods (SWGDAM) guidelines recommend validation studies to determine the reliability of new extraction methods. These recommended studies include sensitivity studies in order to gauge the ability of the extraction method to obtain results from samples with a range of DNA quantities, stability studies intended to investigate the effects of environmental and chemical insults, casetype sample studies performed with sample types encountered in casework, reproducibility studies for the determination of the accuracy and precision of the new method, and studies comparing the new method to established methods.

As mock samples are prepared from fresh epithelial cell samples and wellpreserved semen samples, it is important to examine and assess the effectiveness of this protocol with aged and degraded samples as well as with samples containing inhibitors or found on substrates commonly encountered in casework. Correlation studies for this protocol were performed with post-coital samples preserved for 5 years at $-80^{\circ} \mathrm{C}$ and results compared to those extracted with an established differential extraction method currently used by the Palm Beach County Sherriff's Office (PBSO). Stability studies were conducted with week old samples of known concentration as well as with samples with added bile salts and tannic acid, inhibitors known to bind to DNA and polymerase 
respectively. Sensitivity studies were conducted with samples containing as few as 75 sperm cells. Reproducibility studies appraising the results from sample sets over the course of 3 days were also performed. Samples added to denim jeans, cotton panties, and mixed cotton/polyester bedspread were evaluated to determine the results of this protocol with case-type samples.

\section{B. Materials and Methods}

\section{B.1. Correlation Studies}

A correlation study was performed to evaluate the efficacy of this extraction method through comparison with an existing protocol currently in use by the Palm Beach County Sheriff's Office (PBSO). Post-coital swabs collected in 2011 and stored at $-80^{\circ} \mathrm{C}$ were used for this study. These swabs were documented with detailed information about the volunteer including age range, time since intercourse, time since menstruation, and whether or not her partner had been vasectomized.

Ten swabs from ten individuals were selected and half of each swab was extracted with each method. One sample from one individual was selected to be extracted in triplicate to test for reproducibility.

\section{B.2. Case-type Samples}

Three different materials were examined to determine the ability of the extraction method to obtain suspect profiles from various substrates that may be encountered in casework. Cuttings from denim jeans, a cotton panty, and a 52\% cotton $/ 48 \%$ polyester bedspread were included in this study. Each sample was cut into a 
$1 \mathrm{~cm} \mathrm{X} 1 \mathrm{~cm}$ square. Epithelial cell suspension and sperm cell suspension were added to the sample at a 5:1 epithelial cell and sperm cell ratio with approximately 12,500 epithelial cells ad 2,500 sperm cells in each sample. Samples were obtained from one female and one male volunteer. All swabs were air dried for 1 hour before extraction and processed in triplicate.

\section{B.3. Stability Studies}

Studies were conducted in order to determine the effects of inhibitors and environmental conditions on the results obtained from samples extracted with this method. Inhibitors can negatively affect results by binding to template DNA and preventing amplification or binding to polymerase and blocking its activity. Both types of inhibition can lead to allele dropout. Inhibitors that bind to DNA can lead to an increase in threshold cycle value $(\mathrm{Ct})$ during quantitative polymerase chain reaction (qPCR) due to loss of template as well as changes in melting temperature (Tm) that can be observed as a shift in melt curve. Inhibitors than bind to polymerase tend to decrease efficiency and cause shifts in the exponential slope [68]. It is important to investigate both types of inhibition as they relate to this extraction method.

Bile salts bind to DNA template and inhibit amplification. It has been determined that $50 \%$ qPCR inhibition occurs at $1.25 \mu \mathrm{g} / \mu \mathrm{L}$ and $50 \% 1^{\text {st }}$ allele dropout occurs at 1.2 $\mu \mathrm{g} / \mu \mathrm{L}$ [68]. Tannic acid binds to polymerase blocking its activity. It has been reported that $50 \%$ qPCR inhibition occurs at $15 \mathrm{ng} / \mu \mathrm{L}$ and $50 \% 1^{\text {st }}$ allele dropout occurs at 32 $\mathrm{ng} / \mu \mathrm{L}$ [68]. To test for inhibition effects, one set of swabs was prepared with a 100 
$\mathrm{mg} / \mathrm{mL}$ bile salt solution for $1.5 \mu \mathrm{g} / \mu \mathrm{L}$ of sample. A second set of swabs was prepared with a $1 \mathrm{mg} / \mathrm{mL}$ tannic acid solution for a concentration of $36 \mathrm{ng} / \mu \mathrm{L}$ of sample.

Another set of swab samples was prepared and left at outdoors for one week to determine the effects of environmental conditions. All samples included a 100:1 epithelial cell to sperm cell mixture with 2,500 sperm cells per sample and were prepared in triplicate.

\section{B.4. Sensitivity Studies}

The sensitivity of the extraction method was determined with experiments at low cell concentrations at 20:1 or 5:1 female epithelial cell to sperm cell ratios as well as an experiment at 1:10 to evaluate the protocol with samples containing majority male DNA. Sperm cell counts of 2,500,1,250, 500, 250, 125, and 75 per sample were included in the study. Samples were obtained from one female volunteer and one male volunteer. All samples were added to sterile cotton swabs (Puritan Medical Products Co., Guilford, ME) and air dried for 1 hour prior to extraction. All samples were processed in triplicate.

\section{B.5. Reproducibility Studies}

Reproducibility studies were performed with samples from two female volunteers (E1 and E2) and two male volunteers (S1 and S2). Three sample sets were

tested and designated E1S2, E2S1, and E2S2. All samples were prepared in triplicate and a portion of each swab was sampled over the course of three days. 


\section{B.6. Sample Preparation}

Epithelial cells were collected from healthy female volunteers according to the Institutional Review Board (IRB) of Florida International University recommended protocols. Vaginal swabs were processed in $1.5 \mathrm{~mL}$ tubes in $1 \mathrm{X}$ PBS (pH 7.5) (Fisher Scientific, Fair Lawn, NJ) buffer with agitation for 1 hour. The swabs were transferred to spin baskets (Kerafast, Inc., Boston, MA) and samples were centrifuged at 13,000 rpm for 10 minutes to pellet the epithelial cells. The pellet was diluted with 1X PBS buffer until the target concentration was reached. Sperm cells were obtained from Fairfax Cryobank (Fairfax, VA). The samples were allowed to liquefy at room temperature and samples were diluted with $1 \mathrm{X}$ PBS buffer. Cell counting was performed through microscopic examination of sample injected into a Neubauer-improved disposable C-chip hemocytomer (INCYTO, Fisher Scientific, Fair Lawn, NJ).

\section{B.7. Pressure-based Lysis}

DNA extraction of epithelial cells was achieved through pressure cycling with the Barocycler® NEP 2320 from Pressure BioSciences Inc. (South Easton, MA). Variables were set to 10 cycles with each cycle consisting of 15 seconds at 20,000 psi, and 15 seconds at ambient pressure. All experiments were performed at room temperature. Each sample was placed in a FT500-ND PULSE ${ }^{\text {TM }}$ tube (Pressure BioSciences Inc., South Easton, MA) with $800 \mu \mathrm{L}$ of $0.05 \mathrm{~N} \mathrm{NaOH}$ (Fisher Scientific, NJ) dissolved in molecular biology grade water (Fisher Scientific, NJ). The PULSETM tube was then inserted into the instrument for pressure-based lysis and extraction. Epithelial fractions were then collected by transferring supernatant into a $2 \mathrm{~mL}$ tube and 
transferring the swab into a spin basket. Each sample was then centrifuged at 13,000 rpm for 5 minutes. The pressure-cycling step was performed twice in order to lyse any epithelial cells left behinds after the first run.

\section{B.8. Alkaline Lysis and Neutralization}

The swab was transferred into a $1.5 \mathrm{~mL}$ tube with $400 \mu \mathrm{L}$ of $0.4 \mathrm{~N} \mathrm{NaOH}$. It was then incubated for 5 minutes in a water bath set at $95^{\circ} \mathrm{C}$ for sperm cell lysis. The sperm fraction was collected by transferring the swab into a spin basket with centrifugation proceeding at 13,000 rpm for 5 minutes. The alkaline sample was neutralized with the addition of $160 \mu \mathrm{L}$ of $1 \mathrm{M} \mathrm{HCl}$ prior to purification.

\section{B.9. DNA Purification}

DNA purification was performed with the EZ1 Investigator Kit and the BioRobot EZ1 (Qiagen, Valencia, CA) with the Large-Volume Protocol. Each sample was eluted with $40 \mu \mathrm{L}$ of TE buffer.

\section{B.10. DNA Quantitation}

Amplification and quantitation of DNA extracted from the samples was performed using the Plexor® HY system (Promega Corp., Madison, WI) on the RotorGene 6000 (Corbett, Australia). Quantification of both autosomal and Y-chromosomal DNA was necessary to determine the selectivity of the procedure as well as the optimum amount of sample required for PCR amplification. 


\section{B.11. PCR Amplification and STR Analysis}

The quality of recovered DNA was assessed by performing STR analysis with PowerPlex ${ }^{\circledR} 16$ HS system (Promega Corp., Madison, WI) according to manufacturer's protocols. Samples were amplified using ABI GeneAmp® 9700 thermal cyclers (Applied Biosystems, Foster City, CA). Amplified products were processed using the ABI PRISM $^{\text {TM }} 310$ genetic analyzer and analysis was conducted with the use of GeneMapper ${ }^{\circledR}$ v.4.0 (Applied Biosystems, Foster City, CA) software

\section{Results and Discussion}

\section{C.1. Correlation Studies}

Correlation studies were performed with post-coital swabs collected in 2011 and stored at $-80^{\circ} \mathrm{C}$. Ten samples were selected to be tested with the modified PCT + alkaline lysis protocol and with the PBSO differential extraction method. One sample, PC 175, was selected to be tested in triplicate with both methods. Half of a cutting from each swab was used for extraction. Complete information for all sample is included in Table 1.

Since the genotype of each individual was unknown, selectivity was determined through calculation of peak height ratio of $\mathrm{Y}$ over $\mathrm{X}$ at the Amelogenin locus. A value of 1 should indicate a completely male profile. A sperm cell sample was extracted in triplicate as a control and a $\mathrm{Y}$ over $\mathrm{X}$ ratio of $1.01 \pm 0.04$ was obtained. The results for the ten individual samples are displayed in Figures 36. For most samples, the selectivity achieved was much greater with the PBSO method than with the PCT + alkaline lysis method. Exceptions are observed with PC 158, PC 162, and PC 159. With PC 158, a majority male profile was obtained with PCT + alkaline lysis, but no visible profile could 
be generated with the PBSO method. Greater selectivity was achieved with the PCT + alkaline lysis method with sample PC 162. And the extraction of PC 159 yielded approximately equivalent results. Overall, the average male DNA contribution in the sperm fraction obtained with the PBSO method and PCT + alkaline lysis method was determined to be $0.59 \pm 0.087$ and $0.24 \pm 0.051$ respectively.

\begin{tabular}{|l|l|l|l|}
\hline Sample D & Time since sexual intercourse (hours) & Age range (years) & Time since menstruation (days) \\
\hline PC 158 & $13-18$ & $21-30$ & $8-14$ \\
\hline PC 159 & $13-18$ & $21-30$ & $6-14$ \\
\hline PC 160 & $7-12$ & $31-40$ & $8-14$ \\
\hline PC 161 & $7-12$ & $31-40$ & $8-14$ \\
\hline PC 162 & $7-12$ & $21-30$ & $8-14$ \\
\hline PC 163 & $37-42$ & $31-40$ & $8-14$ \\
\hline PC $175^{*}$ & $13-18$ & $31-40$ & $8-14$ \\
\hline PC 176 & $13-18$ & $31-40$ & $8-14$ \\
\hline PC 177 & $13-18$ & $21-30$ & $8-14$ \\
\hline PC 178 & $13-18$ & $21-30$ & $8-14$ \\
\hline PC 180 & $13-18$ & $31-40$ & $15-21$ \\
\hline
\end{tabular}

Table 1. Correlation study post-coital sample volunteer data. No volunteers reported a vasectomized partner. 


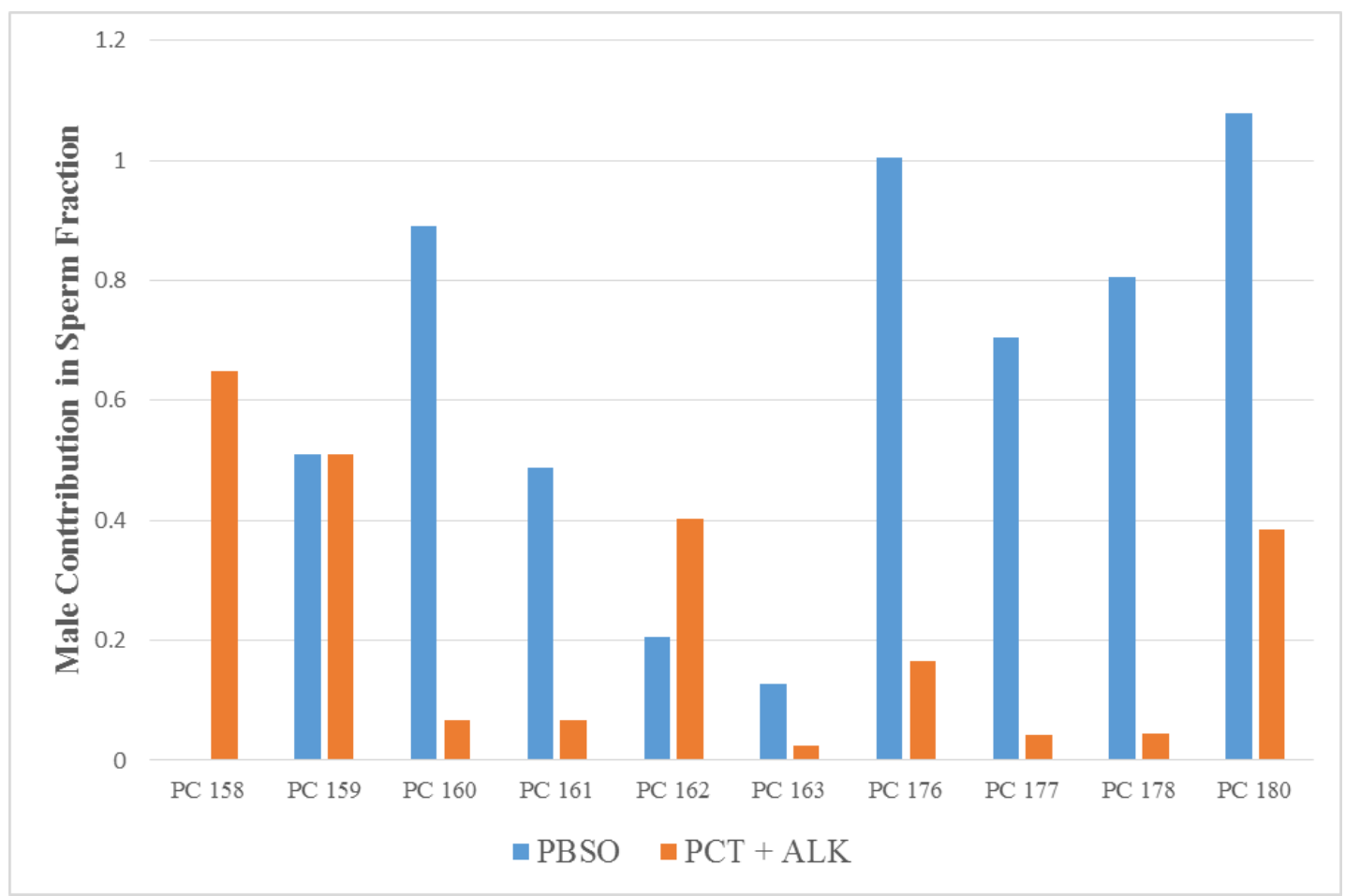

Figure 36. Selectivity comparison between the PBSO and PCT + alkaline lysis method used to extract preserved post-coital samples. PBSO method gave superior results for most samples. Exceptions are observed for PC 158, PC 162, and PC 159. Determined through calculation of peak height ratio of $Y$ over $X$ at the Amelogenin locus.

Sample PC 175 was extracted in triplicate with both methods for the purposes of estimating reproducibility. The results are displayed in Figure 37. Results are similar to those obtained from the individual samples. The PBSO method yielded higher selectivity, $0.60 \pm 0.12$, but greater reproducibility resulted from extraction with PCT + alkaline lysis, $0.24 \pm 0.018$. 


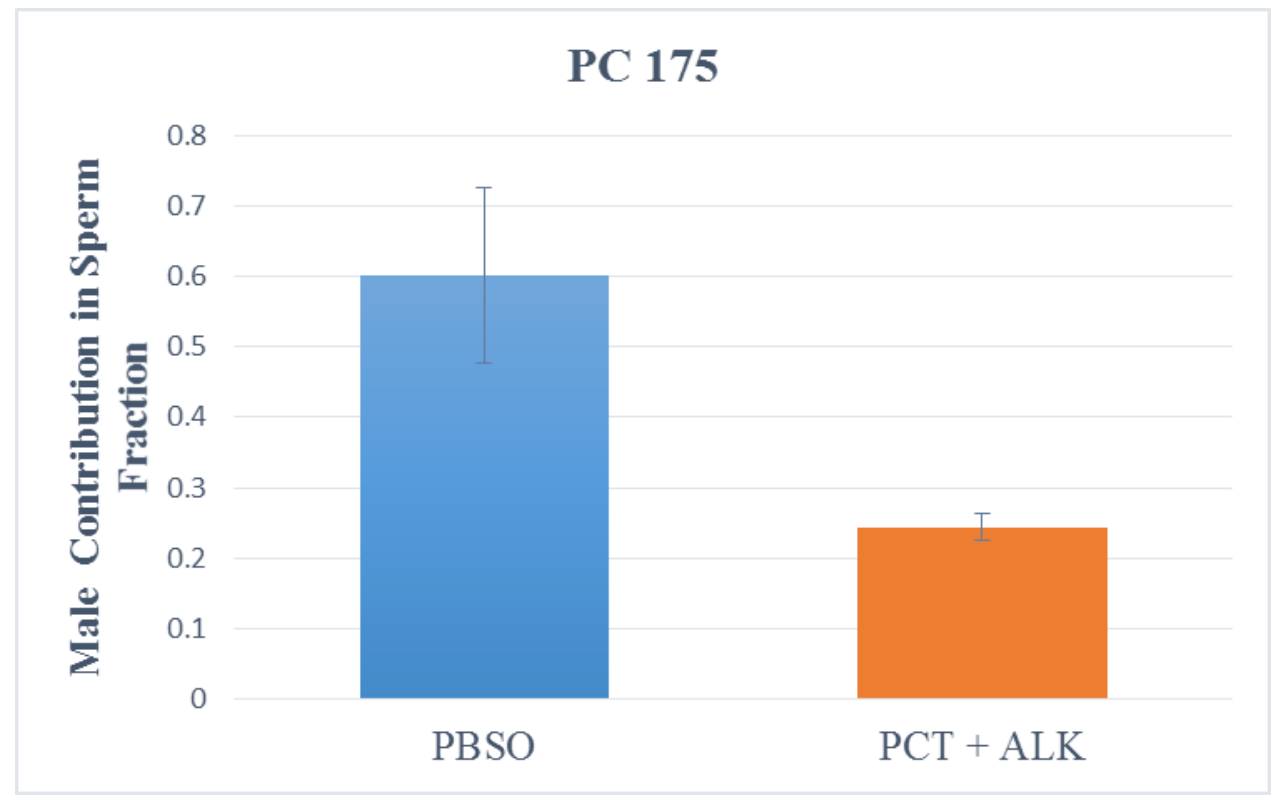

Figure 37. Selectivity comparison between the PBSO and PCT + alkaline lysis method used to extract preserved post-coital samples. PBSO method showed greater male contribution but less reproducibility when compared to PCT + alkaline lysis. Determined through calculation of peak height ratio of $Y$ over $X$ at the Amelogenin locus.

The recovery achieved by both methods was also determined through Plexor ${ }^{\circledR}$ HY quantification of Y-DNA concentration. The results for seven of the ten individual samples tested are displayed in Figure 38. DNA concentrations for samples PC 161, PC 176, and PC 180 exceeded those of other samples and are displayed separately in Figure 39 for the purposes of clarity. Higher recovery was observed with several samples when extracted with the PCT + alkaline lysis method; PC 158, PC 159, PC 162, PC 163, PC 161, and PC 180. Sample PC 175 also resulted in higher recovery with PCT + alkaline lysis with greater reproducibility as seen in Figure 40. The PCT + alkaline lysis method resulted in an average of $723 \pm 495 \%$ more DNA when compared to the PBSO method. 


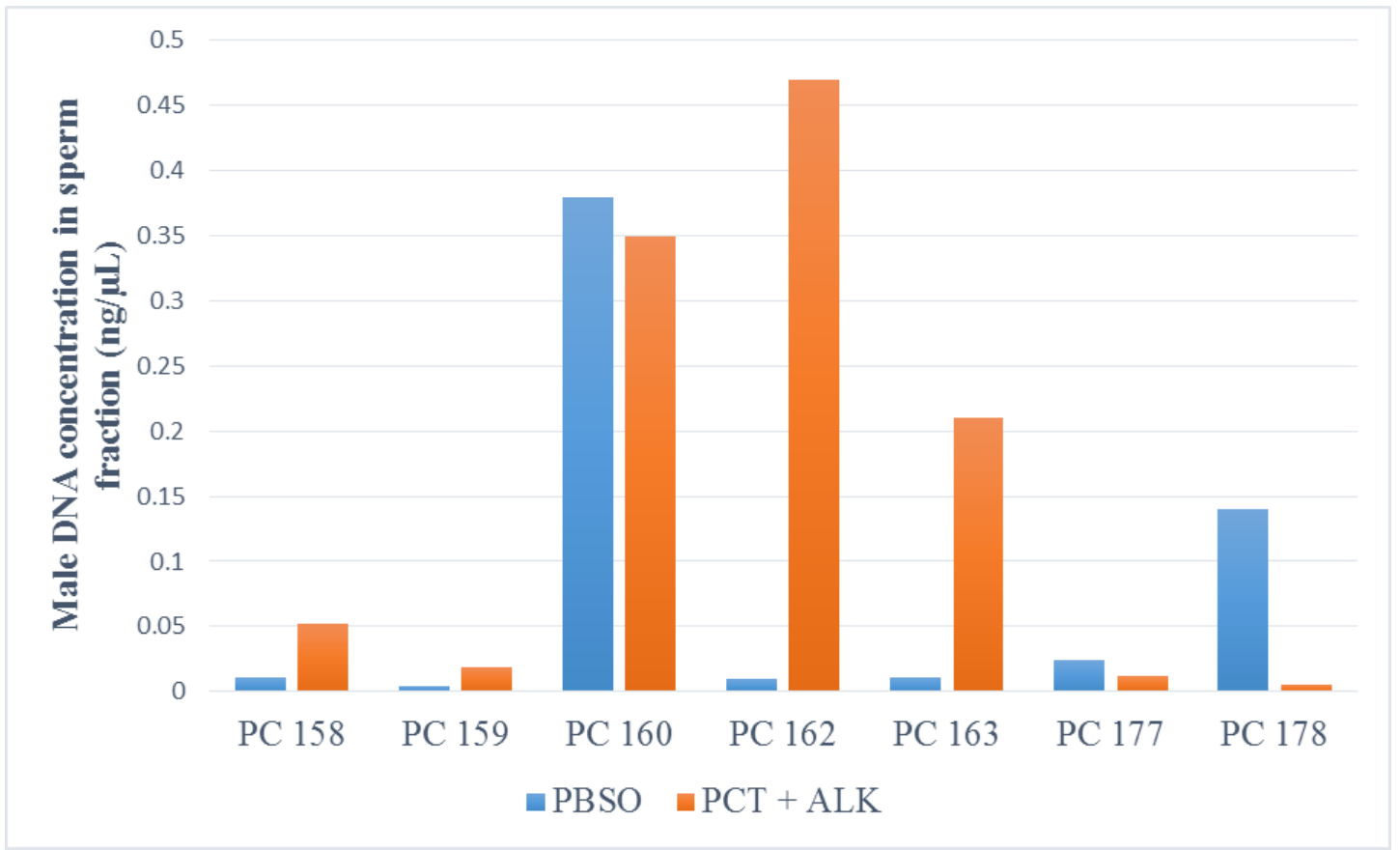

Figure 38. Male DNA concentration in sperm fraction of post-coital samples after extraction with PBSO method or PCT + alkaline lysis method; PC 158, PC 159, PC 160, PC 162, PC 163, PC 177, and PC 177. Higher recovery of male DNA reported with samples PC 158 (373\% increase), PC 159 (342\% increase), PC 162 (4,847\% increase), and PC 163 (1,809\% increase).

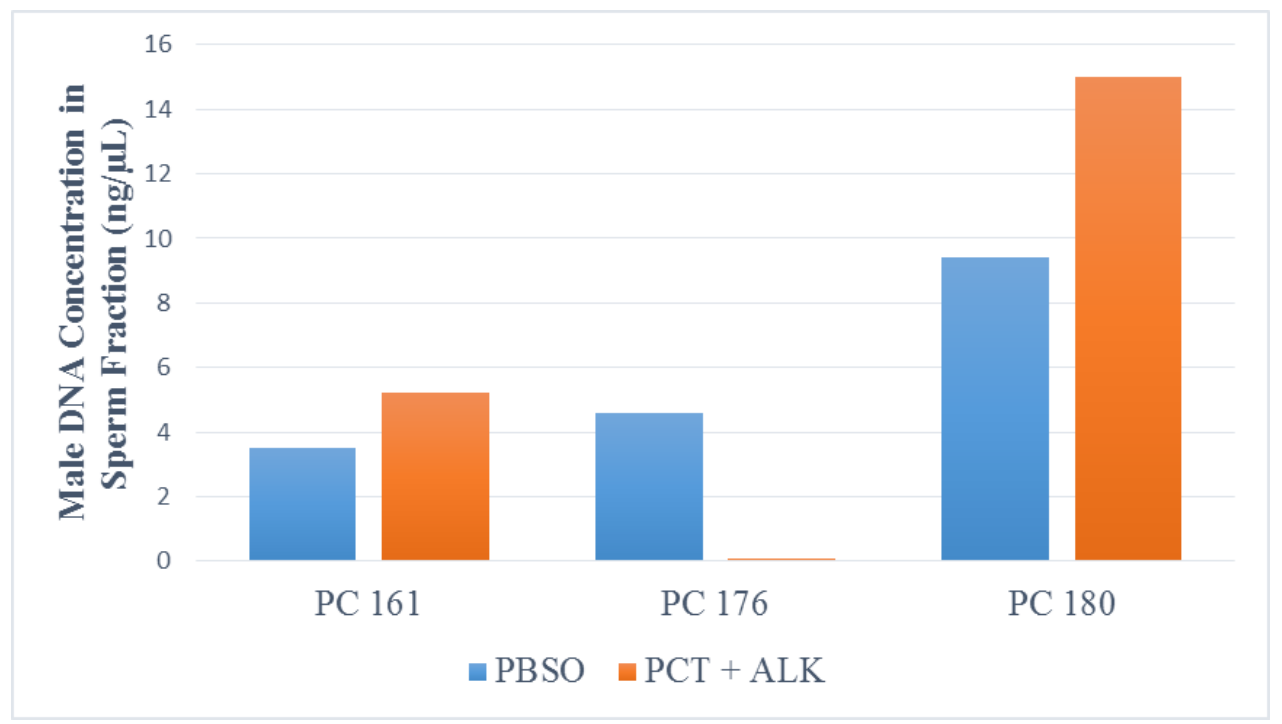

Figure 39. Male DNA concentration in sperm fraction of post-coital samples after extraction with PBSO method or PCT + alkaline lysis method; PC 161, PC 176, and PC 180. Higher recovery of male DNA reported with samples PC 161 (49\% increase) and PC 180 (60\% increase). 


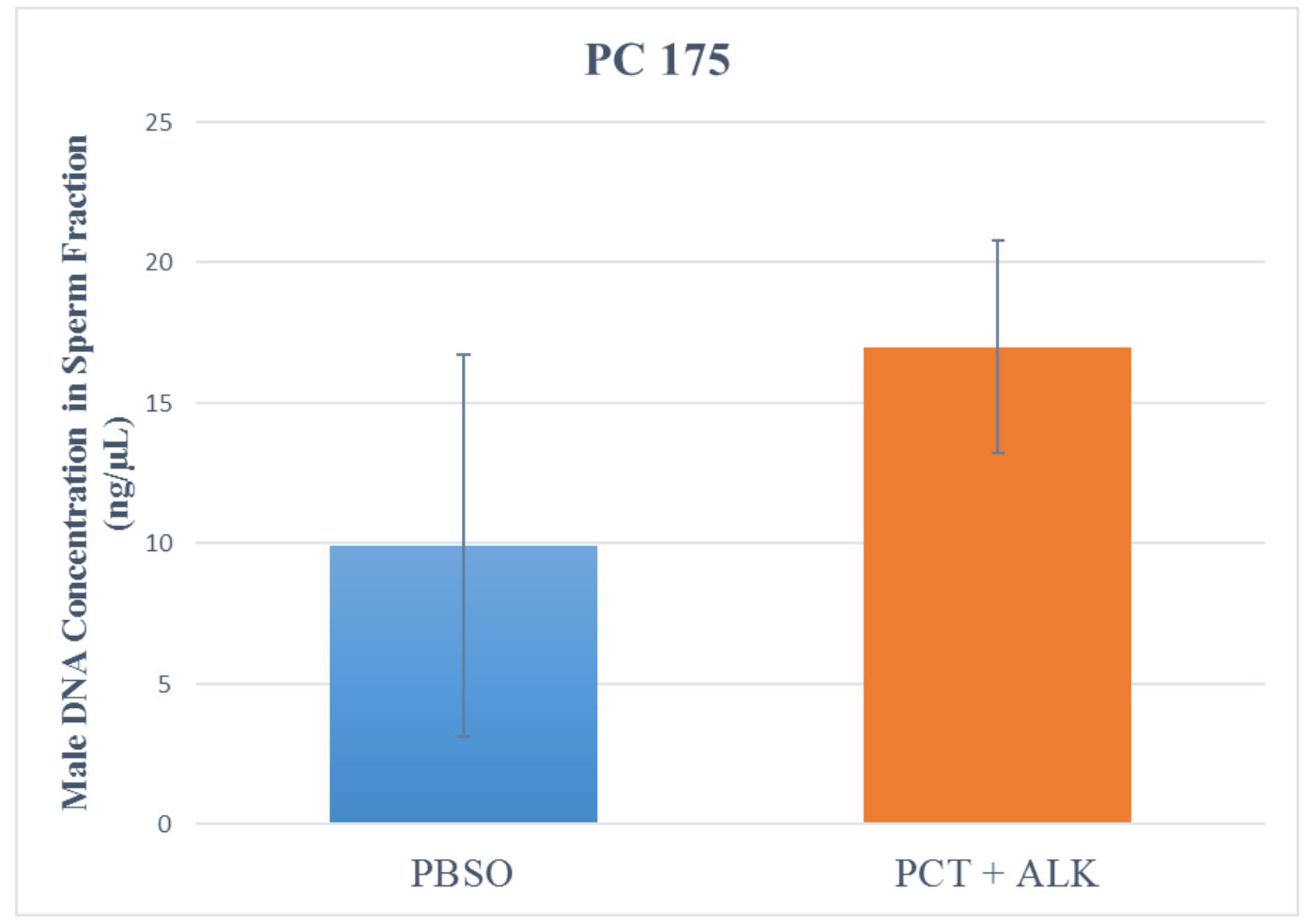

Figure 40. Male DNA concentration in sperm fraction of post-coital sample PC 175 after extraction with PBSO method $(9.9 \pm 6.8 \mathrm{ng} / \mu \mathrm{L})$ or PCT + alkaline lysis method $(17 \pm 3.8$ $\mathrm{ng} / \mu \mathrm{L}) .(\mathrm{n}=3 \pm$ standard error $)$

\section{C.2. Case-type samples}

The sperm fractions extracted from samples deposited on 3 different substrates were compared to those obtained from swabs prepared at with a 5:1 epithelial cell to sperm cell ratio. Cuttings from denim jeans, cotton panties, and a 52\% cotton/48\% polyester bedspread spiked with a 5:1 F:M sample were extracted in triplicate. The results are displayed in Figure 41.

Percent recovery was determined with a $10 \mu \mathrm{L} 250,000$ cell $/ \mathrm{mL}$ sperm cell suspension. Organic extraction was performed by incubating the samples in stain extraction buffer (10 mM Tris, 100mM NaCl, $10 \mathrm{mM}$ EDTA, 2\% SDS, $39 \mathrm{mM}$ DTT) and 
proteinase $\mathrm{K}(20 \mathrm{mg} / \mathrm{ml})$ at $56^{\circ} \mathrm{C}$ for 3 hours followed by phenol-chloroform-isoamyl alcohol purification and ethanol precipitation. Quantitation revealed a concentration of $0.30 \pm 0.016 \mathrm{ng} / \mu \mathrm{L}$. Recovery of male DNA in the sperm fraction of the cotton swab samples was determined to be, $77 \pm 4.0 \%$. The highest recovery among the tested substrates was achieved with the cotton panties at $54 \pm 19 \%$ and lowest with the bedspread with $3.3 \pm 1.2 \%$. The recovery with the denim samples was also quite low at only $8.2 \pm 0.68 \%$

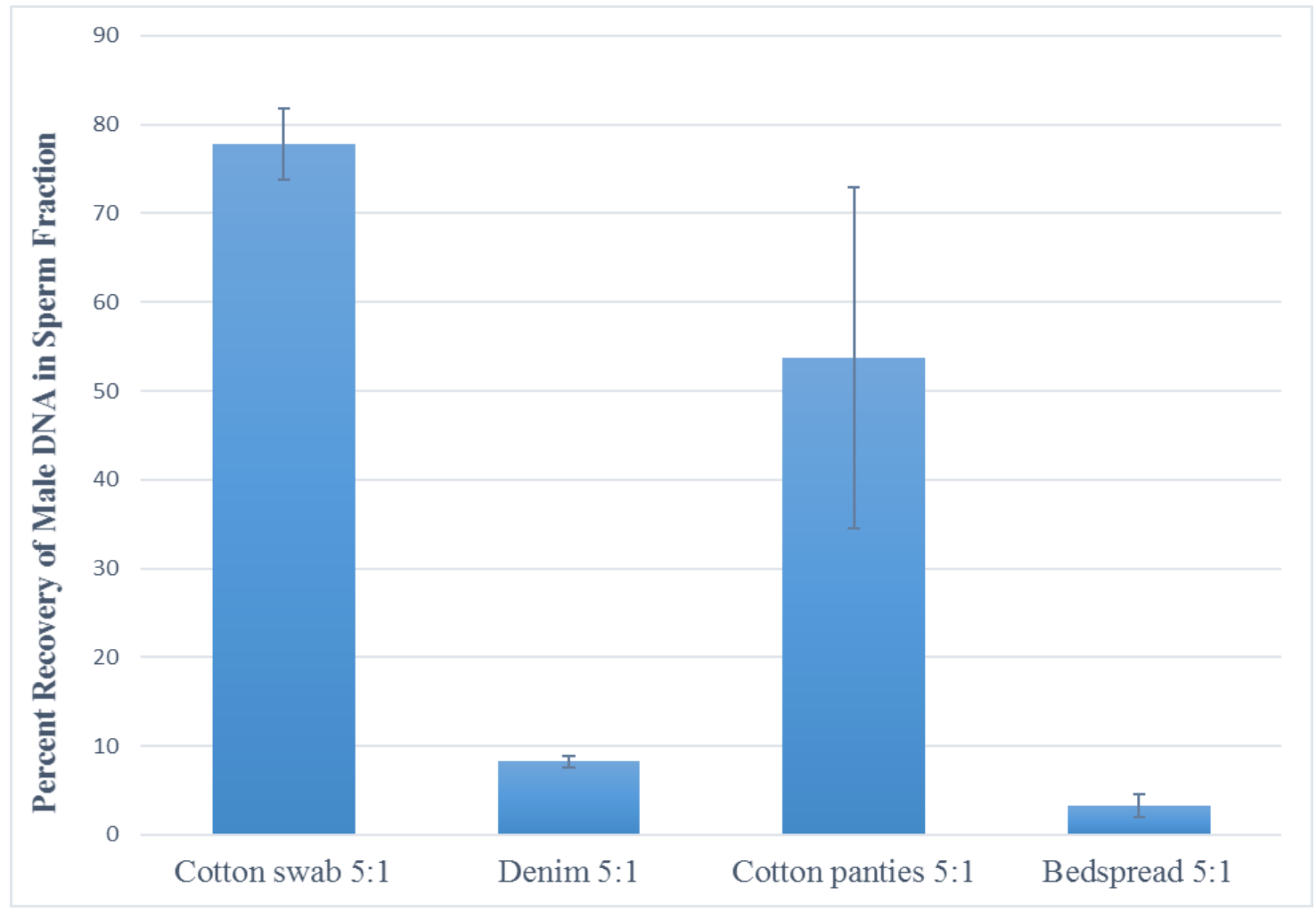

Figure 41. Percent male DNA recovery in sperm fraction on extracted samples deposited on various substrates spiked with 5:1 F:M sample. Recovery was poor in all cases compared to the cotton swab. $(n=3 \pm$ standard error)

The selectivity of the protocol was determined through calculation of male and female contribution to generated STR profiles. A total of seven loci were selected that share 
no alleles in common between the profiles of male and female contributors. Relative Fluorescence Units (RFU) of peaks from male and female contributors were divided by total RFU at each locus x100 for calculation of percentage.

Selectivity results are displayed in Figure 42. Male DNA contribution for samples deposited on denim and cotton panties were lower with a male DNA contribution of $43 \pm 2.8 \%$ and $43 \pm 2.5 \%$ when compared to the cotton swab control, $89 \pm 0.77 \%$. The bedspread sample extraction resulted in a $98 \pm 1.4 \%$ male DNA contribution in the sperm fraction with no signs of male allelic dropout and a negligible amount of contribution from the female profile.

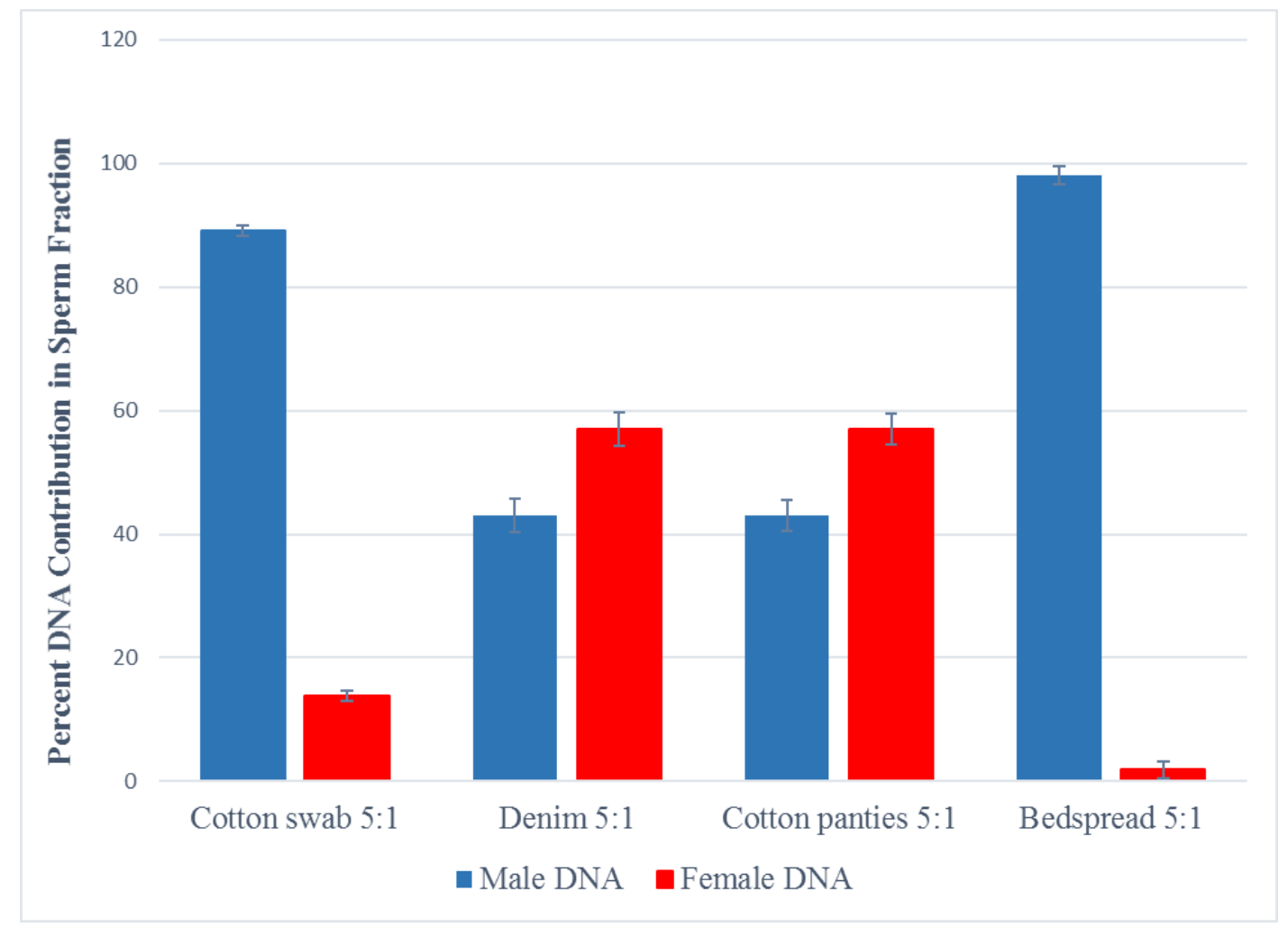

Figure 42. The effect of substrate on selectivity for the sperm fraction from a 5:1 F:M spiked sample. The cotton swab samples resulted in $89 \pm 0.77 \%$ male DNA contribution. Denim and cotton panties resulted in a slight majority female profile with a male DNA contribution of $43 \pm 2.8 \%$ and $43 \pm 2.5 \%$. Nearly all alleles present in the bedspread samples pertained to the 
male contributor with a male DNA contribution of $98 \pm 1.4 \%$. The percent male or female contribution was determined by the male or female peak height divided by total peak height at 7 loci where no alleles were shared between the male or female contributor, $x 100 .(n=3 \pm$ standard error)

\section{C.3. Stability studies}

Selectivity results for 20:1 F:M swab samples treated with $1.5 \mu \mathrm{g} / \mu \mathrm{L}$ bile salts and $36 \mathrm{ng} / \mu \mathrm{L}$ tannic acid are displayed in Figure 43 and compared to 20:1 F:M control samples with a male DNA contribution of $85 \pm 2.4 \%$ in the sperm fraction. Treatment with bile salts and tannic acid did not have a large negative impact on selectivity with male DNA contribution with $82 \pm 1.5 \%$ and $79 \pm 1.6 \%$ respectively.

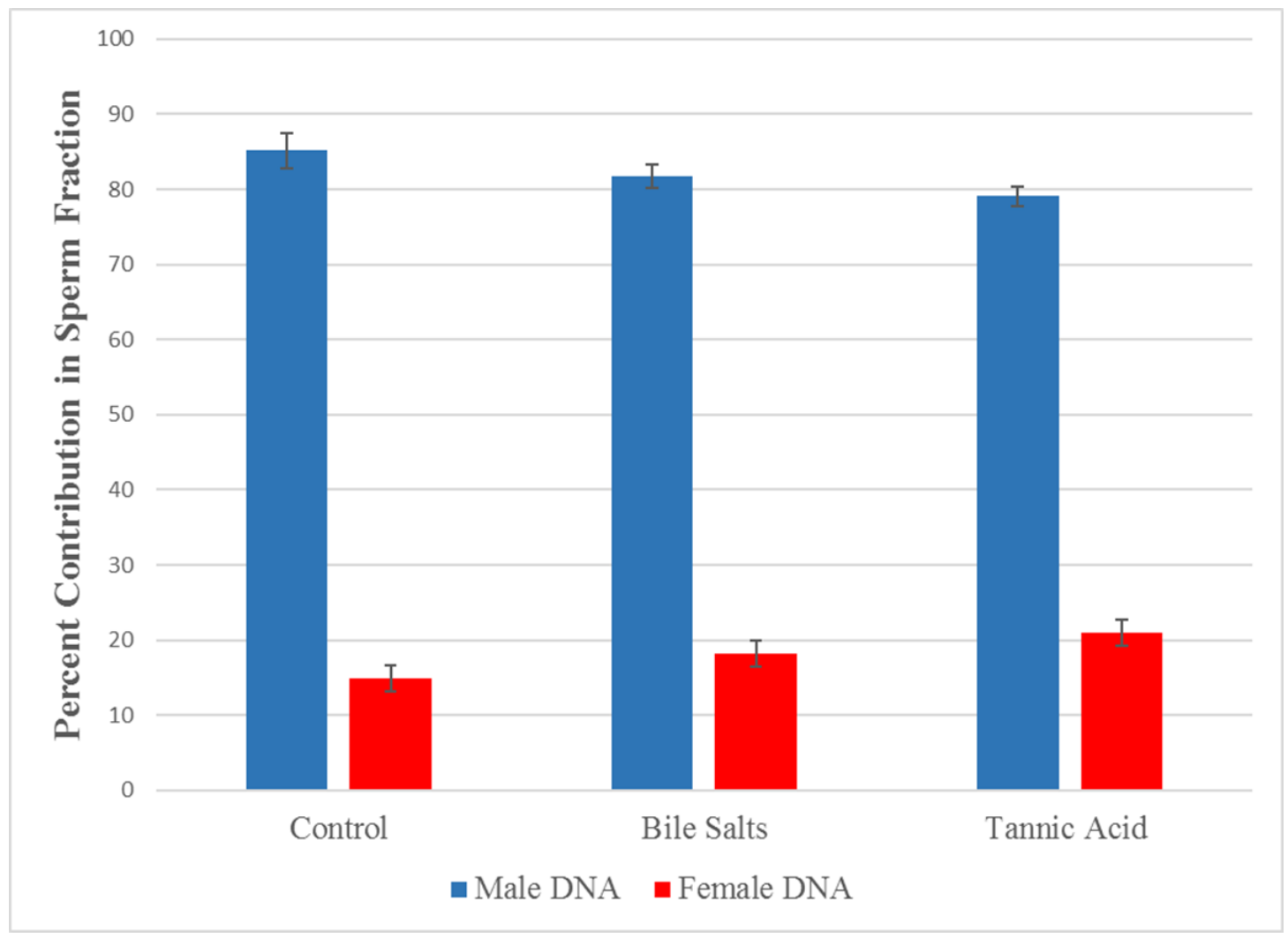

Figure 43. The effect of inhibitors and environmental insults on selectivity of 20:1 F:M sample sperm fractions compared to controls. Bile salts and tannic acid have a minimal effect on selectivity when compared to the control (20:1 F:M on a cotton swab). The percent male or female contribution was determined by the male or female peak height divided by total peak height at 7 loci where no alleles were shared between the male or female contributor, $x 100$. $(n=3 \pm$ standard error $)$ 
Figure 44 compares the recovery of the control sample and samples treated with bile salts or tannic acid. A slight decrease in recovery was detected in samples treated with bile salts with $0.09 \pm 0.027 \mathrm{ng} / \mu \mathrm{L}$ compared to $0.12 \pm 0.031 \mathrm{ng} / \mu \mathrm{L}$, but the standard error is still in range of the control. Tannic acid treated samples yielded better results with $0.11 \pm 0.0058 \mathrm{ng} / \mu \mathrm{L}$.

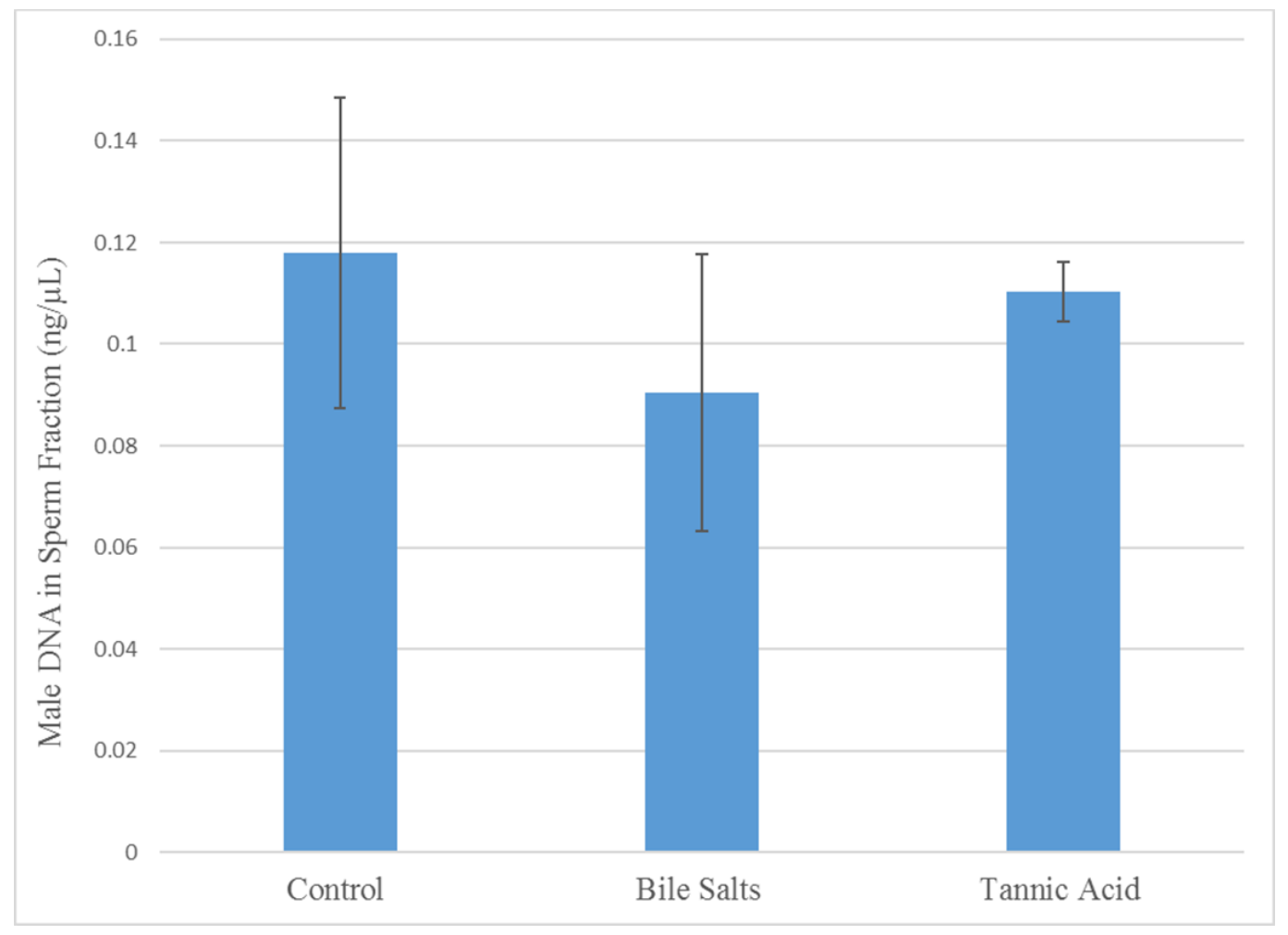

Figure 44. Male DNA concentration in sperm fraction of 20:1 F:M samples treated with either bile salts or tannic acid compared to control. ( $n=3 \pm$ standard error)

Figure 45 displays selectivity results for 100:1 F:M samples subjected to outdoor conditions for 1 week in order to examine the effect of environmental insults. There was only a slight decrease in selectivity observed, with $58 \pm 1.7 \%$ male DNA contribution for control samples and $52 \pm 3.1 \%$ for the outdoor samples. 


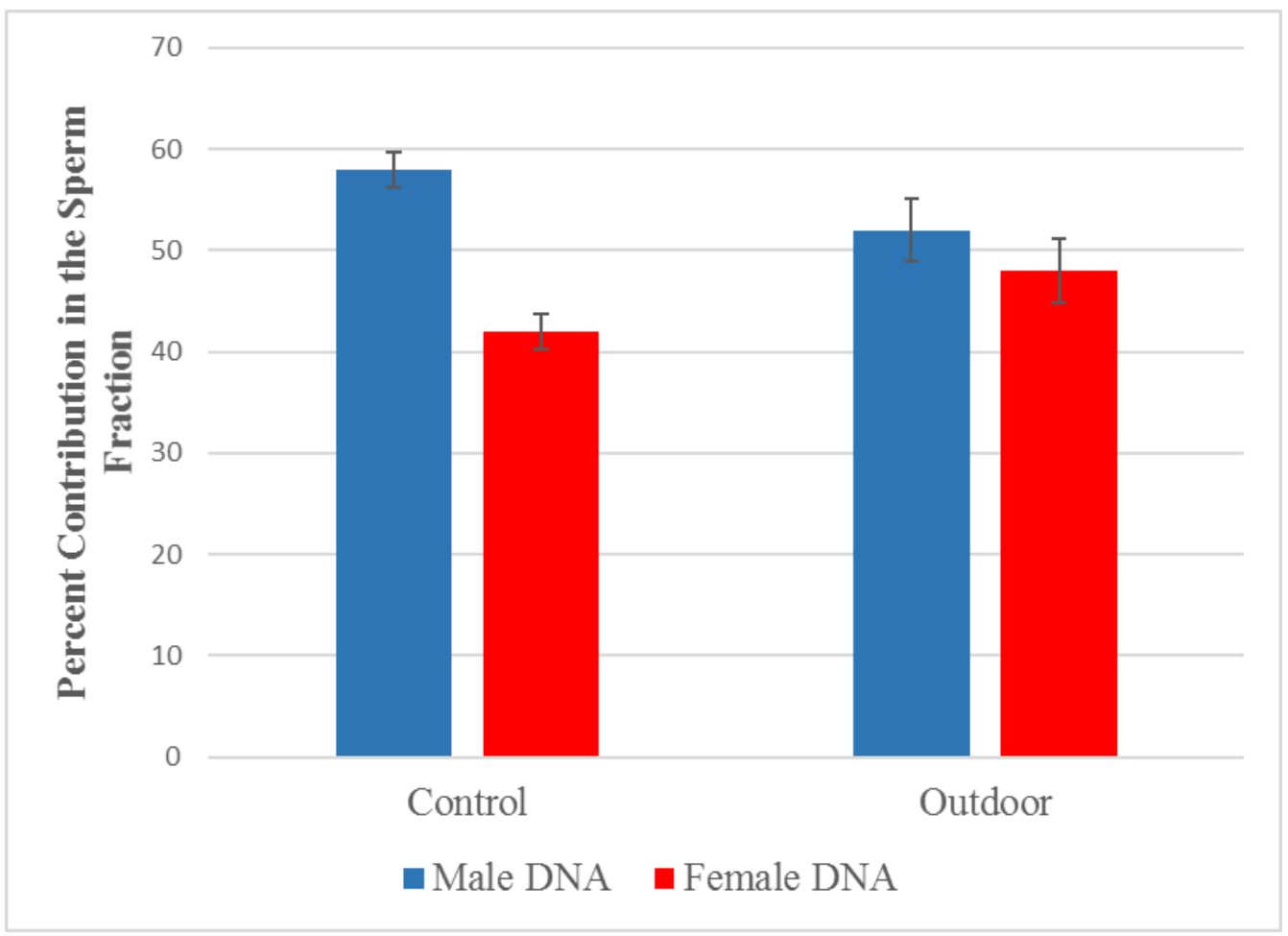

Figure 45. The effect of environmental insults on the selectivity of 100:1 F:M sample sperm fractions compared to controls. A slight decrease form $58 \pm 1.7 \%$ male DNA contribution to $52 \pm 3.1 \%$ is observed. The percent male or female contribution was determined by the male or female peak height divided by total peak height at 7 loci where no alleles were shared between the male or female contributor, $x 100$. $(n=3 \pm$ standard error)

Figure 46 compares the recovery of male DNA found in the sperm fraction between the control and outdoor samples. The concentration of male DNA was determined to be $0.16 \pm 0.017 \mathrm{ng} / \mu \mathrm{L}$ in the control samples and $0.13 \pm 0.036$ in the outdoor samples. Although a decrease in recovery was observed, possibly the result of male DNA loss during the PCT step, the standard error of the outdoor samples is within range of the controls. 


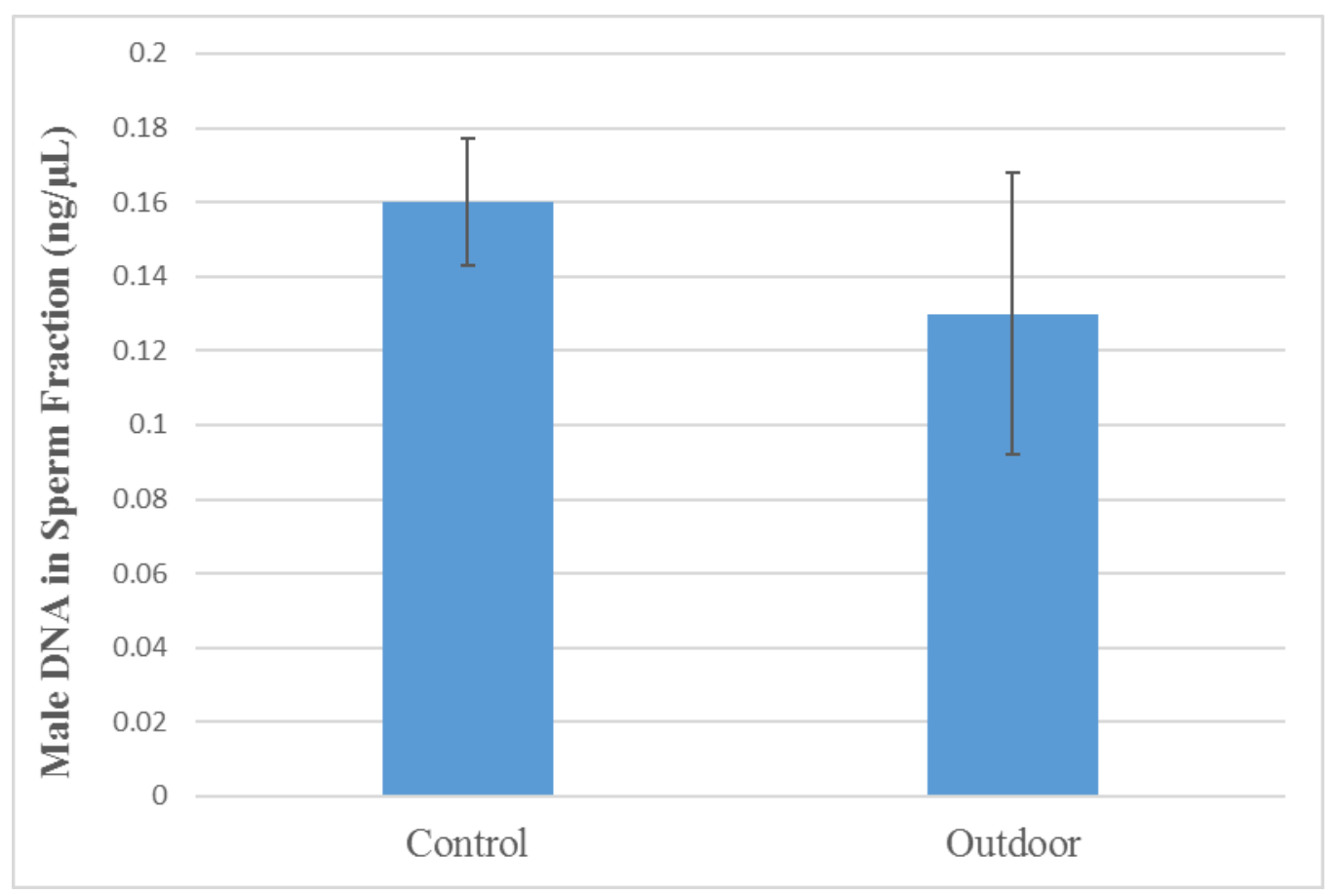

Figure 46. Male DNA concentration in the sperm fraction of 100:1 F:M samples subjected to outdoor environmental conditions for 1 week compared to control. $(n=3 \pm$ standard error $)$

\section{C.4. Sensitivity Studies}

The sensitivity of the extraction method was determined with the preparation of various samples at a 20:1 female epithelial cell to sperm cell ratio with a decreasing number of cells. Selectivity was determined through calculation of peak height ratio of Y over $\mathrm{X}$ at the Amelogenin locus. Figure 47 and Table 2 contain full details and results. Unfortunately, as the number of cells dropped, the male DNA contribution decreases. Figures 48-52 depict STR profiles obtained at each concentration. It should be noted that even with only 125 sperm cells present in the sample, all male alleles are visible with 14 of 16 loci above containing male alleles above 150 RFUs. 


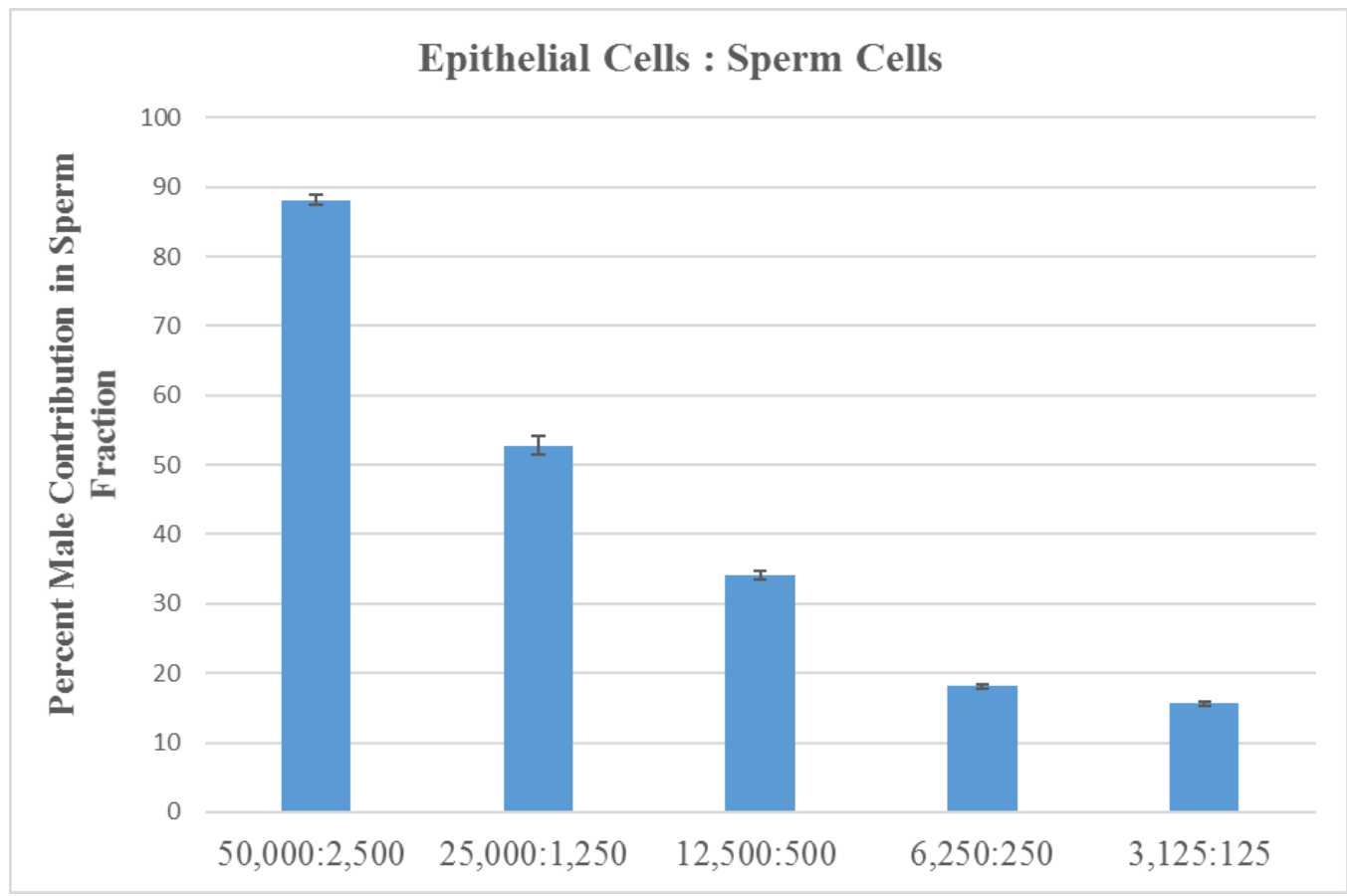

Figure 47. Selectivity of developed protocol with decreasing number of cells present in sample. Male DNA contribution decreased with decreasing cell count. Percentage determined by male peak height divided by total peak height at 7 loci where no alleles are shared between the male or female contributor, $x 100$. $(n=3 \pm$ standard error $)$

\begin{tabular}{|l|l|l|l|}
\hline Epithelial cells : Sperm cells & $\begin{array}{l}\text { Percent Male Contribution } \\
\text { in Sperm Fraction } \\
(\mathbf{n}=\mathbf{3} \pm \text { standard error) }\end{array}$ & $\begin{array}{l}\text { Male alleles detected above } \\
\mathbf{1 5 0} \text { RFUs per locus }\end{array}$ & $\begin{array}{l}\text { Major } \\
\text { Contributor }\end{array}$ \\
\hline $50,000: 2,500$ & $89 \pm 0.81$ & $16 / 16$ & Male \\
\hline $25,000: 1,250$ & $53 \pm 1.3$ & $16 / 16$ & Male \\
\hline $12,500: 500$ & $34 \pm 0.61$ & $16 / 16$ & Female \\
\hline $6,250: 250$ & $18 \pm 0.33$ & $16 / 16$ & Female \\
\hline $3,125: 125$ & $16 \pm 0.25$ & $14 / 16$ & Female \\
\hline $375: 75$ & $65 \pm 4.0$ & $7 / 16$ & Mixed \\
\hline $250: 2,500$ & $92 \pm 0.25$ & $16 / 16$ & Male \\
\hline
\end{tabular}

Table 2. Sensitivity studies data table. 


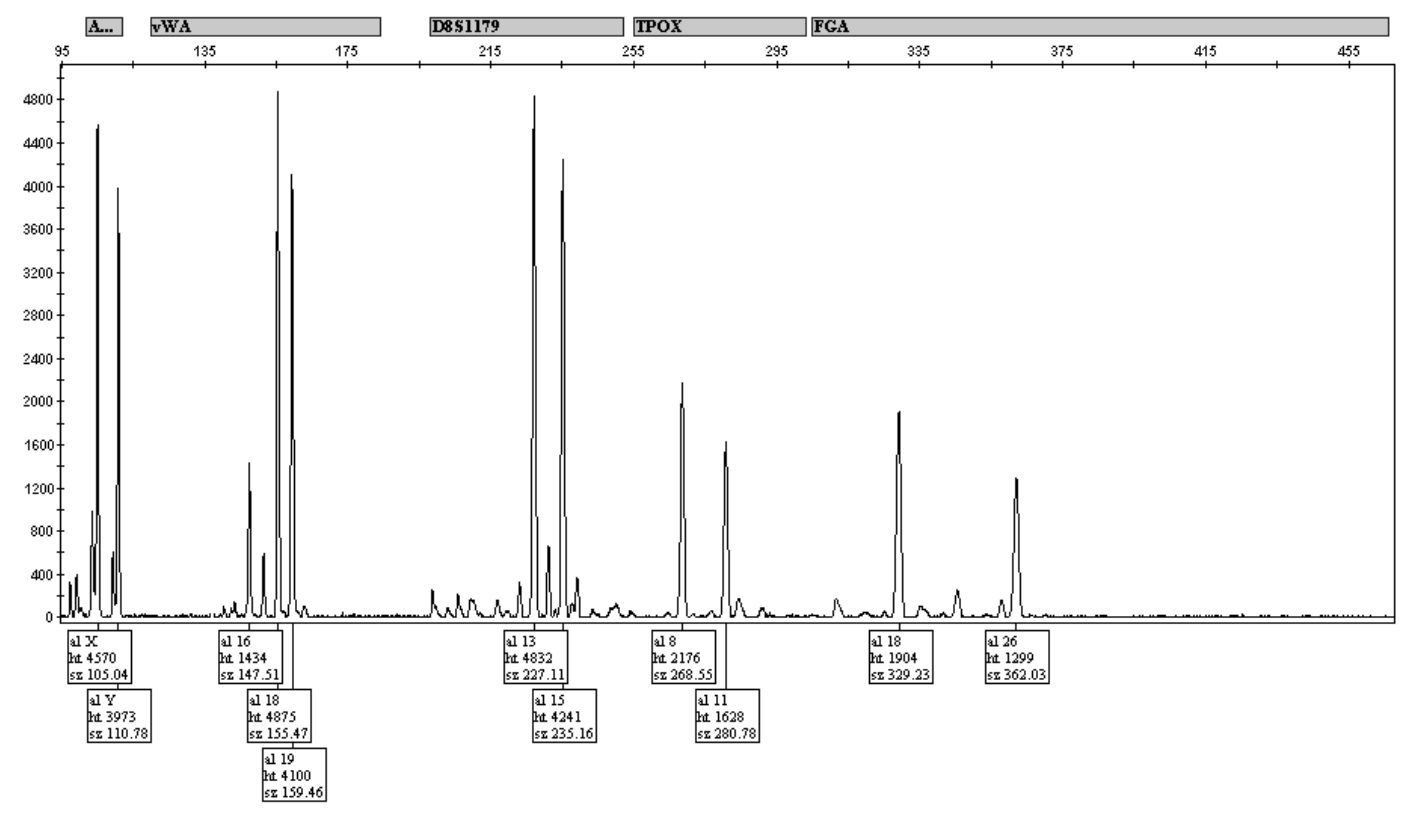

Figure 48. Sperm fraction profile of a 50,000:2,500 epithelial cell:sperm cell sample. A percent male DNA contribution of $90 \%$ is observed in the profile. Panel represents loci labeled with 5-TAMRA (5-Carboxytetramethylrhodamine); Amelogenin, D8S1179, TPOX, and FGA.

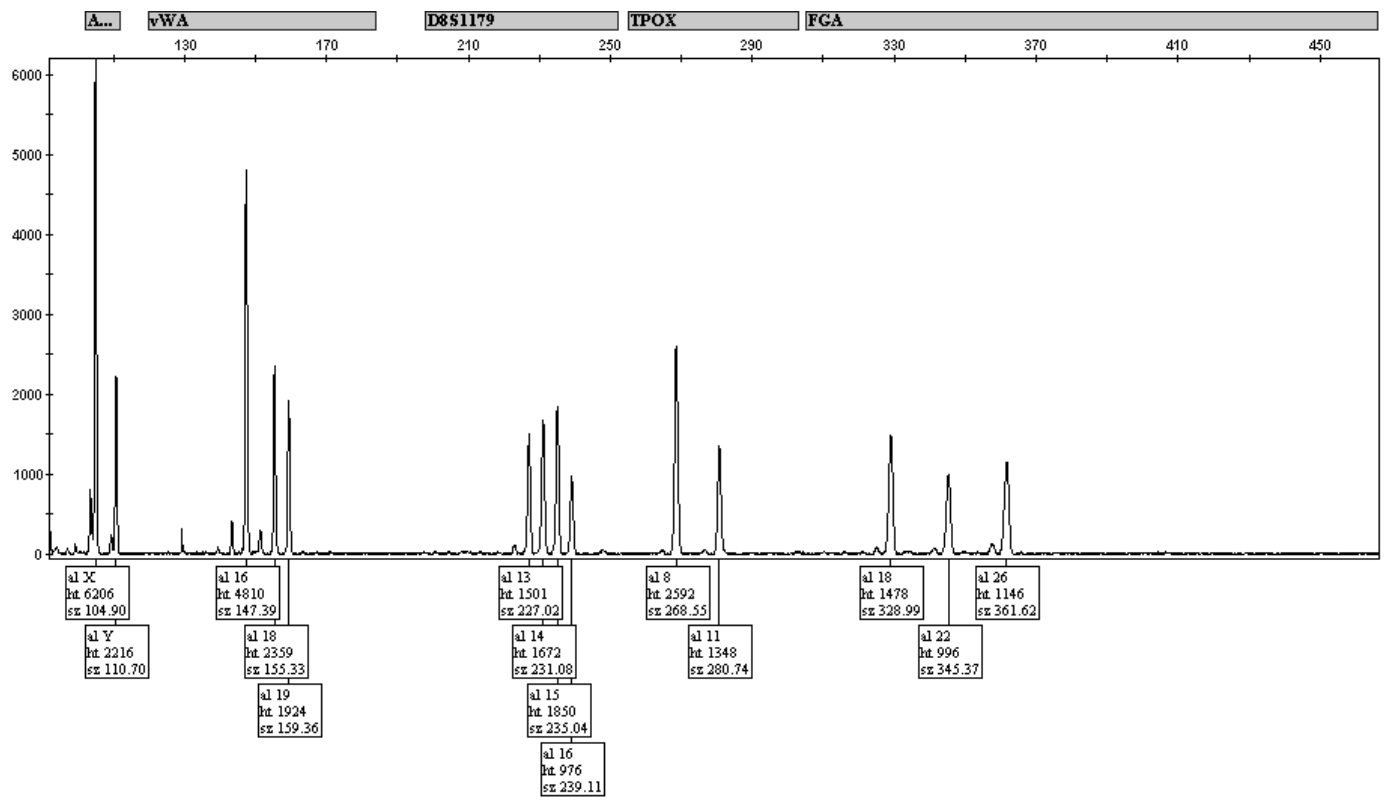

Figure 49. Sperm fraction profile of a 25,000:1,250 epithelial cell:sperm cell sample. A percent male DNA contribution of $55 \%$ is observed in the profile. Panel represents loci labeled with 5-TAMRA (5-Carboxytetramethylrhodamine); Amelogenin, D8S1179, TPOX, and FGA. 


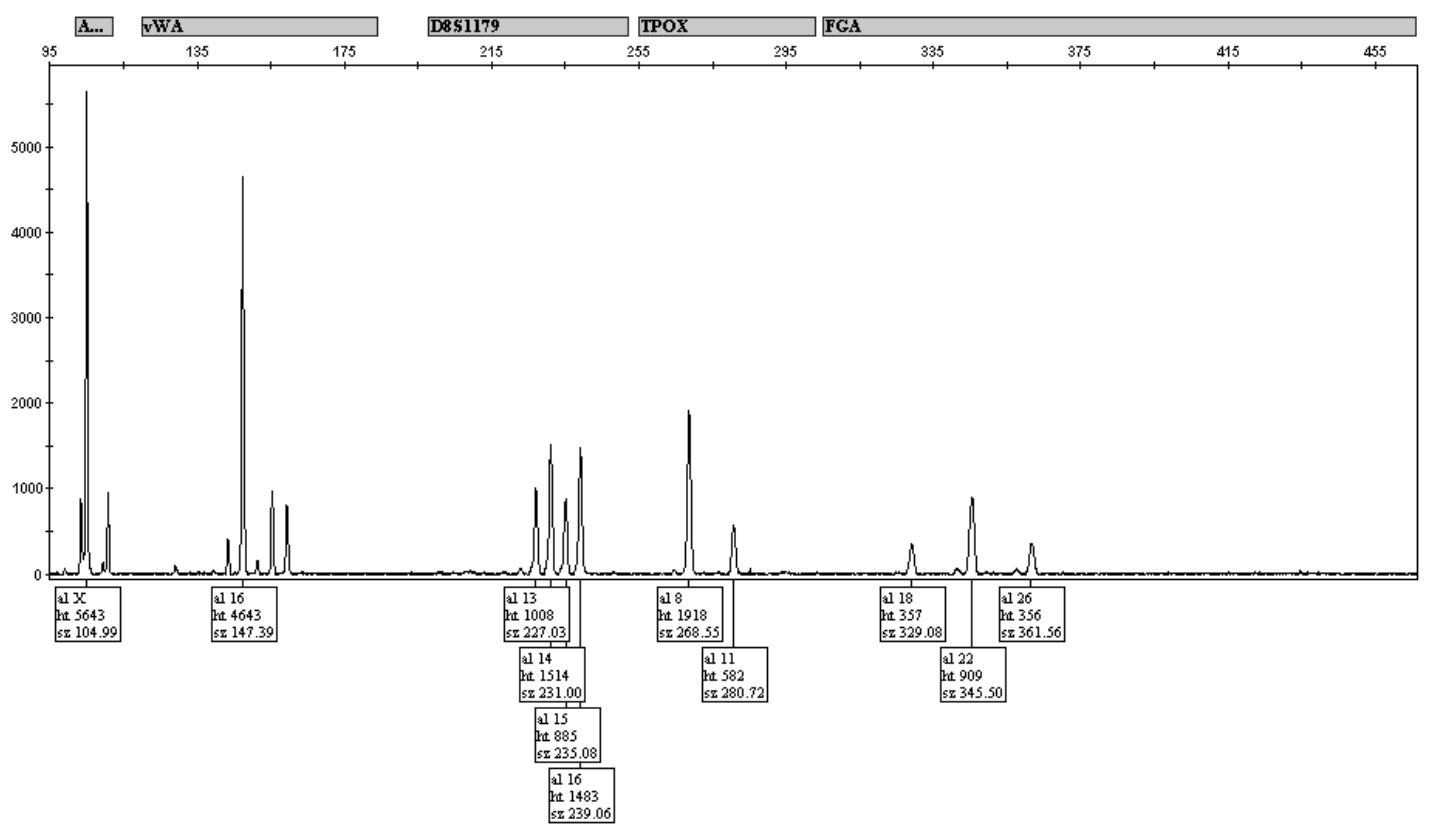

Figure 50. Sperm fraction profile of a 12,500:500 epithelial cell:sperm cell sample. A percent male DNA contribution of $36 \%$ is observed in the profile. Panel represents loci labeled with 5-TAMRA (5-Carboxytetramethylrhodamine); Amelogenin, D8S1179, TPOX, and FGA.

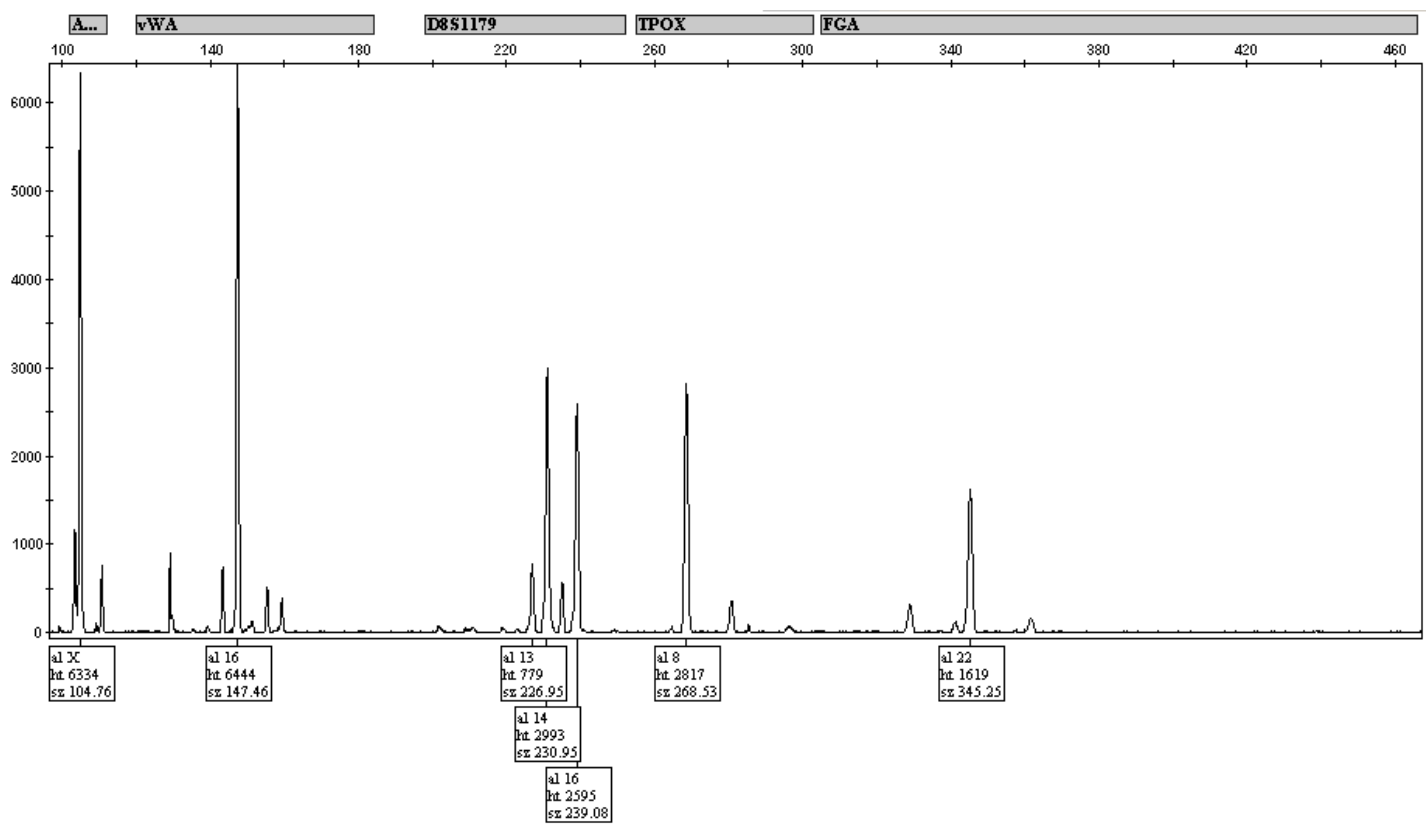

Figure 51. Sperm fraction profile of a 6,250:250 epithelial cell:sperm cell sample. A percent male DNA contribution of $17 \%$ is observed in the profile. Panel represents loci labeled with 5-TAMRA (5-Carboxytetramethylrhodamine); Amelogenin, D8S1179, TPOX, and FGA. 


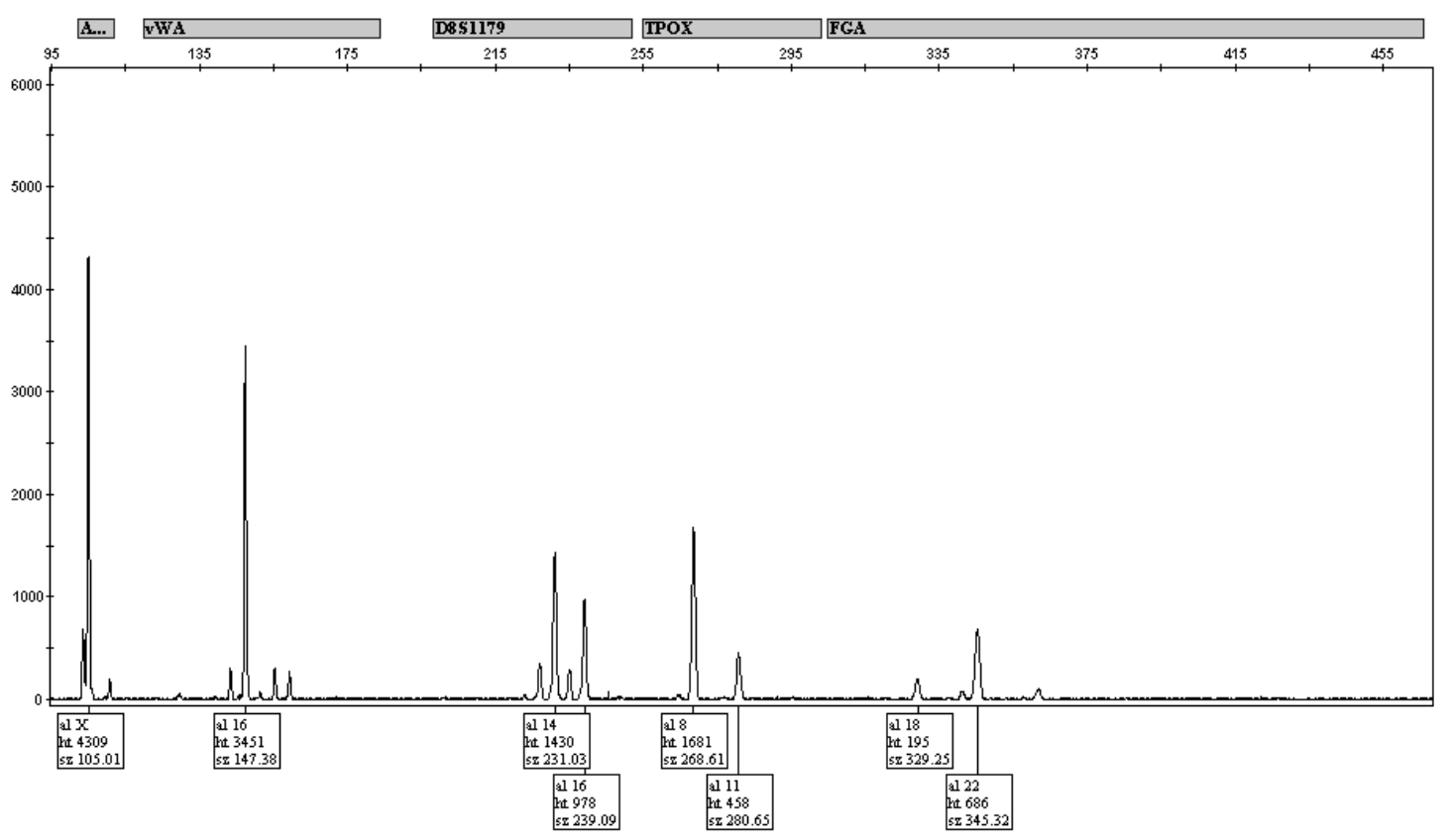

Figure 52. Sperm fraction profile of a 3,125:125 epithelial cell:sperm cell sample. A percent male DNA contribution of $16 \%$ is observed in the profile. Panel represents loci labeled with 5-TAMRA (5-Carboxytetramethylrhodamine); Amelogenin, D8S1179, TPOX, and FGA.

The protocol was also tested at a 5:1 female epithelial cell to sperm cell ratio with only 75 sperm cells. The results are reported in Table 2 the STR profile is displayed in Figure 53. 


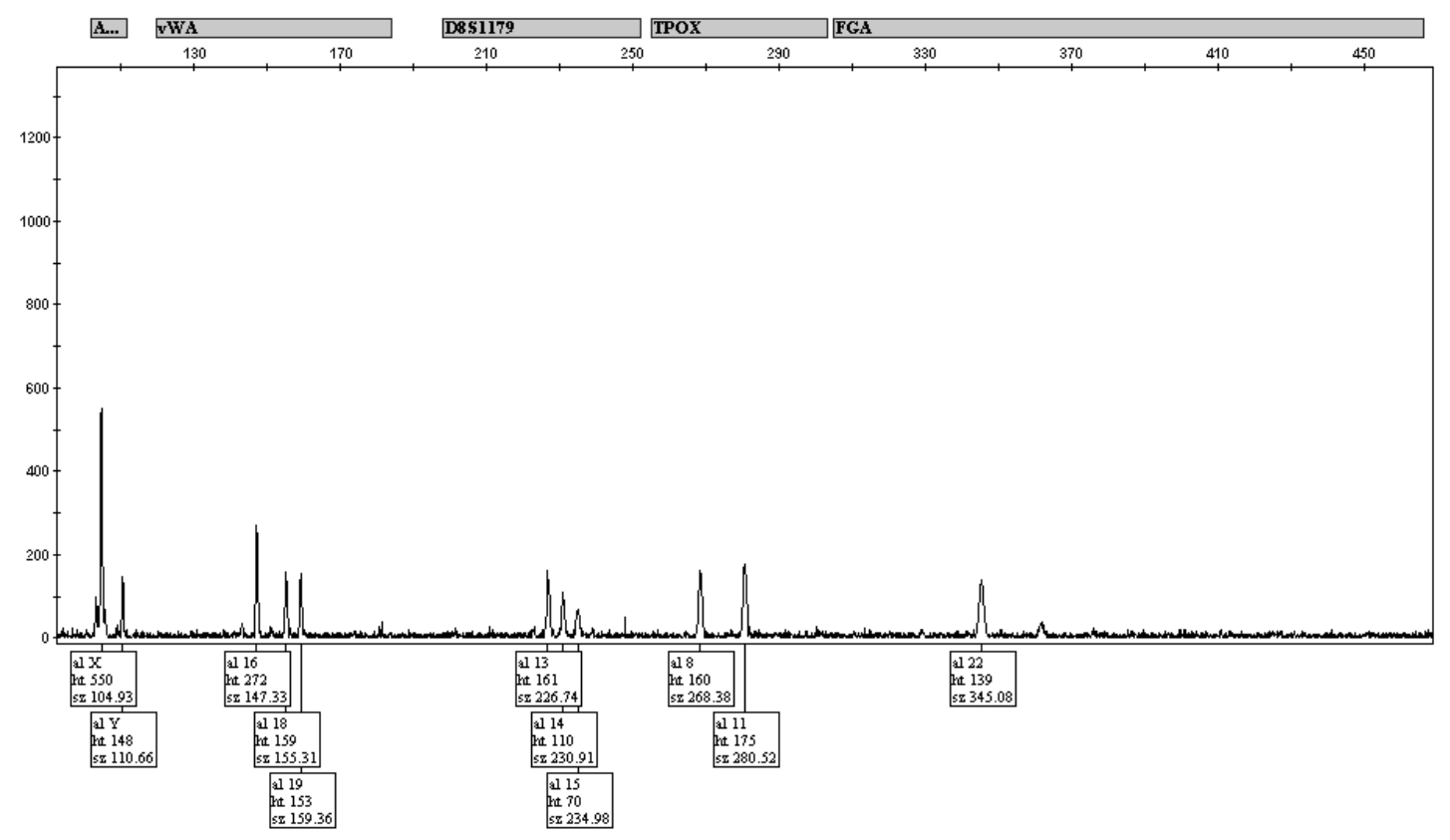

Figure 53. Sperm fraction profile of a 375:75 epithelial cell:sperm cell sample. A percent male DNA contribution of $66 \%$ is observed in the profile. Dropout occurred at the FGA locus. Panel represents loci labeled with 5-TAMRA (5-Carboxytetramethylrhodamine); Amelogenin, D8S1179, TPOX, and FGA.

Encouragingly, even with such a low amount of DNA, all male alleles of 14 of the 16 loci and Amelogenin were visible although only 7 loci contained all male alleles above 150 RFUs.

The protocol was also examined with a sample containing a majority amount of male DNA. The sample prepared at 1:10 female epithelial cell to sperm cell ratio with 250 epithelial cells and 2,500 sperm cells. The Y/X peak height ratio is surprisingly low at only $0.72 \pm 0.0041$, but as can be seen in the STR panel in Figure 54, this is the result of a peak imbalance and the STR profile is clearly male. 


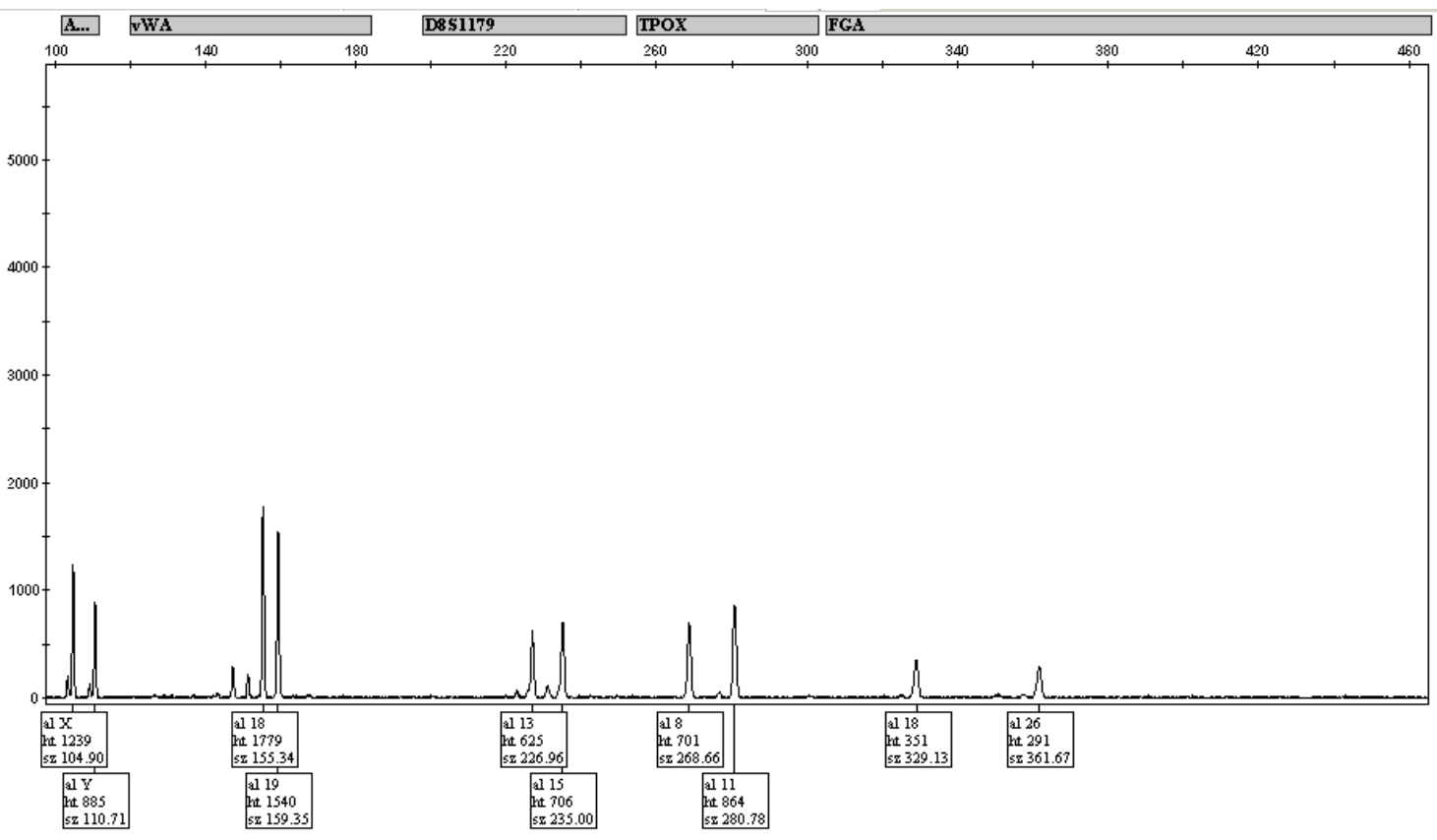

Figure 54. Sperm fraction profile of a 250:2,500 epithelial cell:sperm cell sample. A percent male DNA contribution of $94 \%$ is observed in the profile. Panel represents loci labeled with 5-TAMRA (5-Carboxytetramethylrhodamine); Amelogenin, D8S1179, TPOX, and FGA.

\section{C.5. Reproducibility Studies}

Figure 55 and Table 3 represents the male DNA obtained for each sample set at each day of the reproducibility study. The sample sets E1S1, E1S2, and E2S2 resulted in averages of $0.28 \pm 0.044 \mathrm{ng} / \mu \mathrm{L}, 0.39 \pm 0.064 \mathrm{ng} / \mu \mathrm{L}$, and $0.34 \pm 0.045 \mathrm{ng} / \mu \mathrm{L}$. Recovery of male DNA varied by a significant extent day by day with results from the first day showing far less recovery, with only $18 \pm 0.077 \mathrm{ng} / \mu \mathrm{L}$ for E1S1, $0.19 \pm 0.036 \mathrm{ng} / \mu \mathrm{L}$ for E1S2, and 0.19 $\pm 0.054 \mathrm{ng} / \mu \mathrm{L}$ for E2S2. 


\begin{tabular}{|l|l|l|l|l|}
\hline & Day 1 & Day 2 & Day 3 & Mean \\
\hline E1S1 & $0.18 \pm 0.077$ & $0.32 \pm 0.069$ & $0.33 \pm 0.13$ & $0.28 \pm 0.044$ \\
\hline E1S2 & $0.19 \pm 0.036$ & $0.55 \pm 0.058$ & $0.45 \pm 0.092$ & $0.39 \pm 0.064$ \\
\hline E2S2 & $0.19 \pm 0.054$ & $0.46 \pm 0.015$ & $0.37 \pm 0.038$ & $0.34 \pm 0.045$ \\
\hline
\end{tabular}

Table 3. Male DNA (ng/ $\mu \mathrm{L})$ present in sperm fraction for sample sets E1S1, E1S2, and E2S2 at each day of the experiment.

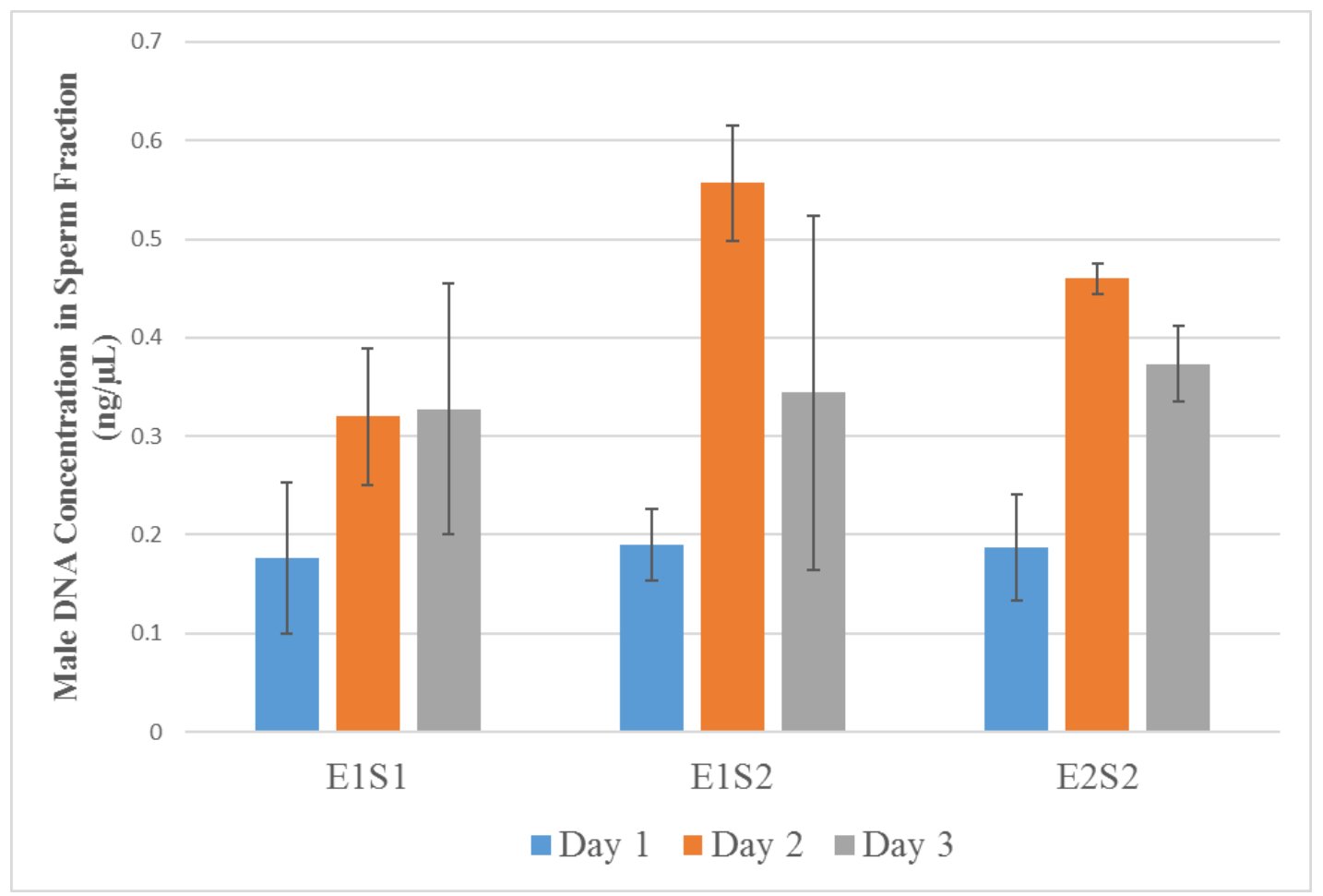

Figure 55. Reproducibility of male DNA recovery among three sets of samples over three days. $(n=3 \pm$ standard error)

Reproducibility of the selectivity of the modified protocol fared better over the course of the 3 days as depicted in Figure 56 and Table 4. Selectivity was determined through calculation of peak height ratio of $\mathrm{Y}$ over $\mathrm{X}$ at the Amelogenin locus and male peak height over total peak height at the D871179 locus, $x$ 100. E1S1 and E2S2 showed little variation in selectivity with average male DNA contributions of $72 \pm 7.0 \%$ and 
$77 \pm 6.6 \%$. E1S2 with an average of $74 \pm 1.6 \%$ showed more variation, particularly between

Day 1 and Day 2 with a difference of $57 \pm 9.8 \%$ and $94 \pm 2.5 \%$.

\begin{tabular}{|l|l|l|l|l|}
\hline & Day 1 & Day 2 & Day 3 & Mean \\
\hline E1S1 & $68 \pm 16$ & $71 \pm 12$ & $79 \pm 13$ & $72 \pm 7.0$ \\
\hline E1S2 & $57 \pm 9.8$ & $94 \pm 2.5$ & $79 \pm 9.0$ & $77 \pm 6.6$ \\
\hline E2S2 & $76 \pm 2.8$ & $75 \pm 3.1$ & $72 \pm 3.0$ & $74 \pm 1.6$ \\
\hline
\end{tabular}

Table 4. Percent Male DNA contribution present in sperm fraction for sample sets E1S1, E1S2, and E2S2 at each day of the experiment.

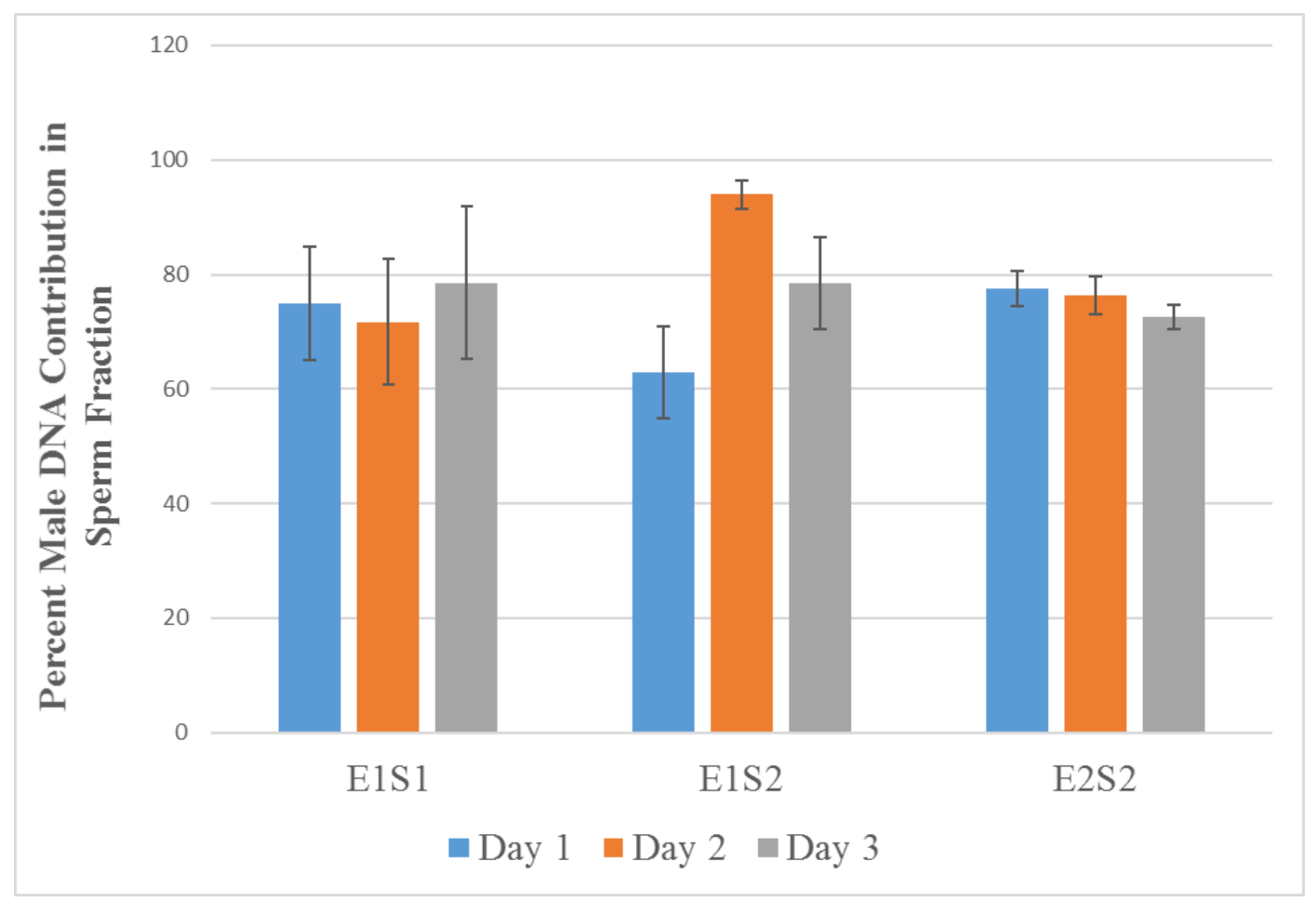

Figure 56. Reproducibility of selectivity among three sets of samples over three days. Results were more reproducible for set E1S1 and E2S2 compared to E1S2. Selectivity was determined through calculation of peak height ratio of $Y$ over $X$ at the Amelogenin locus and male peak height over total peak height at the D871179 locus, $x$ 100. ( $n=3 \pm$ standard error) 


\section{Concluding Remarks}

The PCT + alkaline lysis method resulted in improved recovery with a number of post-coital samples when compared to the established method used by Palm Beach County Sheriff's Office. Unfortunately, this result was at the expense of selectivity which was lower on average compared to the established method. The PCT + alkaline lysis protocol was designed and optimized with the used of entire swab heads for more accurate determination of percent recovery. In order to completely cover the substrate, $800 \mu \mathrm{L}$ of $\mathrm{NaOH}$ are added during the PCT step. Such a large volume used with smaller substrates such as swab cuttings may result in inefficient transmission of pressure to the sample.

The case-type sample study revealed poor recovery for samples deposited on the three tested substrates when compared to cotton swabs. The bedspread substrate was a thin material with low absorbency. This possibly resulted in efficient recovery of epithelial cell DNA and the loss of most sperm cell DNA during the PCT step. The small amount left behind consisted almost entirely of male DNA leading to high selectivity. A single round of pressure cycling may be sufficient for such samples deposited on nonabsorbent substrates. The low selectivity observed with the samples deposited on denim and cotton panties indicate a different problem. Denim and cotton are both rather absorbent materials and epithelial cells were left behind after the PCT step. As with swab cuttings, the small size of the substrate and high volume of $\mathrm{NaOH}$ used during the PCT step may have led to an inefficient transmission of pressure to the substrate and low

recovery of epithelial cell DNA. The lower recovery of male DNA from the denim 
compared to the cotton panties may be caused by sperm cells trapped in the thick denim material or inhibition from the indigo dye.

Stability studies with both bile salts and tannic acid show little decrease in efficiency in the presence of inhibitors. Marshall et al. reported a decrease in inhibition effects in the presence of hematin and humic acid with samples subjected to pressure cycling. The mechanism behind these results are still under investigation [66]. Alkaline lysis has also been known to dilute inhibitors present in a sample [54]. Environmental effects appear to have only resulted in a minor decrease in selectivity of the extraction method with $58 \pm 1.7 \%$ male DNA contribution in controls to $52 \pm 3.1 \%$ in samples subjected to outdoor conditions for one week. It is possible that sperm cells were damaged leading to some male DNA loss during the PCT step.

Male alleles are detectable even in numbers as low as 125 or 75 cells, but selectivity suffers with low DNA samples. These results may be due in part to sperm cells being left behind in the swab decreasing the male DNA contribution in the sperm fraction.

Swab sample recovery does not appear to be particularly reproducible, but this does not have a large negative impact on selectivity which remained largely consistent from day to day. 
CHAPTER VIII. IMMUNOMAGNETIC CAPTURE (IMC) OF EPITHELIAL

\section{CELLS}

\section{A. Introduction}

When performing differential extractions, the key issue is providing sufficient selectivity in a binary mixed sample to permit determination of the male contributor's full genotype. An alternative method to perform this step involves cell specific capture. Cell capture techniques have been attempted with sperm specific antigens in hopes of obtaining a pure sperm fraction and a clear suspect profile. Difficulty with dried and older samples have proven to be impediments to this approach. Antigens located on the neck and mid-piece of the sperm cell become less effectives as in dried samples these sections detach from the head containing the DNA [6]. Experiments with older samples have shown that antigen stability tends to deteriorate over time [60]. More recent experiments with anti-PH-20 immunomagnetic beads have shown more promise, but still required selective degradation with DNase for a clear profile when working with samples containing large number of epithelial cells [118].

An alternative approach is the use of commercial kits for epithelial cell immunomagnetic capture. These kits have been developed for the separation of epithelial cells for breast cancer research [70], but this method have not yet been explored for forensic purposes. Such a scheme would involve removal of epithelial cells in order to achieve a male DNA isolate. This technique of negative selection of epithelial cells is unlikely to yield clear suspect profiles by itself. However, it is possible that if used as a pretreatment to differential extraction methods, this procedure may remove sufficient 
epithelial cells to produce an improvement in selectivity. Thus for this study the potential of immunomagnetic cell capture (IMC) pretreatment was examined as a method to further improvement of the selectivity of pressure cycling and alkaline lysis extraction protocol.

\section{Materials and Methods}

\section{B.1. Cell Suspension Preparation}

Epithelial cells were collected from healthy female volunteers according to the institutional review board (IRB) of Florida International University recommended protocols. Vaginal swabs were processed in $1 \mathrm{~mL}$ of $1 \mathrm{X}$ PBS buffer (pH 7.5) (Fisher Scientific, Fair Lawn, NJ) with 1 hour of agitation. The samples were transferred to spin baskets (Kerafast, Inc., Boston, MA) and centrifuged at 13,000 rpm for 10 minutes to obtain a cell pellet, which was then diluted to the desired concentration. Sperm samples were obtained from Fairfax Cryobank (Fairfax, VA) and allowed to liquefy at room temperature. The samples were then also diluted to the desired concentration with $1 \mathrm{X}$ PBS buffer.

\section{B.2. Cell Count and Sample Preparation}

Cell counting was accomplished through microscopic examination with Neubauer-improved disposable C-chips (INCYTO, Fisher Scientific, Fair Lawn, NJ). Mock samples were prepared with the addition of epithelial cell and sperm cell suspensions to a sterile cotton swab (Puritan Medical Products Co., Guilford, ME). The samples were then allowed to air dry for 1 hour prior to extraction. Samples were prepared containing; 250,000 epithelial cells and 1,250 sperm cells; 250,000 epithelial cells and 
2,500 sperm cells; 125,000 epithelial cells and 2,500 sperm cells; for epithelial cell to sperm cell ratios of 200:1, 100:1, and 50:1 respectively. All samples were prepared in triplicate. The procedure was tested with fresh samples prepared from epithelial cell suspension stored for fewer than 5 days at $4^{\circ} \mathrm{C}$. Stability was tested with a post-coital sample stored at $-80^{\circ} \mathrm{C}$ for 5 years.

\section{B.3. Immunomagnetic Capture (IMC) of Epithelial Cells}

Stemcell Technologies produces the EasySep ${ }^{\mathrm{TM}}$ Human EpCam Positive Selection Kit (Vancouver, Canada), an immunomagnetic cell capture kit that is designed for capturing human mammary epithelial cells. This kit was used to capture excess female epithelial cells prior to pressure cycling.

The swab was inserted into a $5 \mathrm{~mL}$ BD Falcon ${ }^{\mathrm{TM}}$ tube (Franklin Lakes, NJ) with $500 \mathrm{~mL}$ of Robosep ${ }^{\mathrm{TM}}$ Buffer (Stemcell Technologies, Vancouver, Canada). This was followed by the addition of $50 \mu \mathrm{L}$ of antibody cocktail and 20 minutes of incubation on ice. Then, $25 \mu \mathrm{L}$ of dextran-coated magnetic particle suspension was added, followed by 15 more minutes of incubation ice. Additional RoboSep buffer was pipetted into the sample for a total volume of $2.5 \mathrm{~mL}$. The tube was then inserted into the EasySep ${ }^{\mathrm{TM}}$ magnet (Stemcell Technologies, Vancouver, Canada) for 5 minutes. The swab was removed and snipped into a FT500-ND PULSETM tube (Pressure BioSciences Inc., South Easton, MA). The supernatant was poured out of the tube that was still contained in the magnet. The captured epithelial fraction remained in the $5 \mathrm{~mL}$ tube and was saved for analysis. 


\section{B.4. Pressure Cycling Technology and Pressure-based Lysis}

DNA extraction proceeded at room temperature with the Barocycler NEP 2320 from Pressure BioSciences, Inc. (South Easton, MA). Swabs were snipped with sterilized scissors into FT500-ND PULSETM tubes with $800 \mu \mathrm{L} 0.05 \mathrm{~N} \mathrm{NaOH}$ prepared from sodium hydroxide crystals (Fisher Scientific, NJ) dissolved in molecular biology grade water (Fisher Scientific, NJ). Pressure-cycling was set at 10 cycles with each cycle consisting of 15 seconds at 20,000 psi and 15 seconds at ambient pressure. The supernatant was pipetted into a $2 \mathrm{~mL}$ tube and the swab was transferred to a spin basket. Centrifugation of the sample proceeded at 13,000 rpm for 5 minutes for elution and collection of the epithelial fraction. This pressure lysis step was repeated once more for the lysis and removal of remaining epithelial cells.

\section{B.5. Alkaline Lysis and Neutralization}

The swab was placed in a $1.5 \mathrm{~mL}$ tube with $400 \mu \mathrm{L}$ of $0.4 \mathrm{~N} \mathrm{NaOH}$ for sperm cell lysis at $95^{\circ} \mathrm{C}$ for 5 minutes. It was transferred to a spin basket and the sample was centrifuged at 13,000 rpm for 5 minutes for collection of the sperm fraction. The alkaline sample was neutralized with the addition of $160 \mu \mathrm{L}$ of $1 \mathrm{M} \mathrm{HCl}$ (Fisher Scientific, NJ).

The sperm fraction and captured epithelial cells were extracted with the use of an organic extraction method that involved the addition of $300 \mu \mathrm{L}$ of lysis buffer $(10 \mathrm{mM}$ Tris, $100 \mathrm{mM} \mathrm{NaCl}, 10 \mathrm{mM}$ EDTA, $2 \%$ SDS, $39 \mathrm{mM}$ DTT) with $2 \mu \mathrm{L}$ of Proteinase K solution (Promega Corp., Madison, WI). Following incubation at $56^{\circ} \mathrm{C}$ for $2-4$ hours, the samples were purified using phenol chloroform isoamyl alcohol (25:24:1 v/v) (SigmaAldrich, St. Louis, MO). DNA was then precipitated with 3 M sodium acetate and 100\% 
ethanol. After centrifugation, the pellet was washed twice with 70\% ethanol and finally diluted in 1X Tis-EDTA (TE) buffer (Sigma-Aldrich, St.Louis, MO).

\section{B.6. DNA Quantitation}

Quantitation of both autosomal and Y-chromosomal DNA was performed with the Plexor ${ }^{\circledR}$ HY System (Promega Corp., Madison, WI) according to manufacturer's guidelines and with the Rotor-Gene 6000 (Corbett, Australia). The female DNA concentration in the captured epithelial fraction was determined by subtraction of quantified Y-DNA from quantified autosomal DNA.

\section{B.7. PCR Amplification and STR Analysis}

The PowerPlex ${ }^{\circledR} 16$ HS System (Promega Corp., Madison, WI) was used for PCR amplification with the ABI GeneAmp ${ }^{\circledR} 9700$ thermal cycler (Applied Biosystems, Foster city, CA). STR profiles were generated with the ABI PRISM ${ }^{\mathrm{TM}} 310$ Genetic Analyzer (Applied Biosystems, Foster City, CA). Samples were diluted in 9.5 $\mu \mathrm{L} \mathrm{Hi-}$ $\mathrm{Di}^{\mathrm{TM}}$ formamide (Applied Biosystems, Foster city, CA) and $0.5 \mu \mathrm{L}$ ILS 600 Size Standard (Promega Corp., Madison, WI). The subsequent results were analyzed with GeneMapper $^{\circledR}$ v.4.0 (Applied Biosystems, Foster City, CA) software.

\section{Results and Discussion}

\section{C.1. IMC Cell Ratio Test}

The selectivity of the protocol was determined through calculation of male and female contribution to generated STR profiles. Seven loci were selected that shared no alleles in common between the profiles of male and female contributors. Relative 
Fluorescence Units (RFU) of peaks from male and female contributors were divided by total RFU at each locus x100 for calculation of percentage.

The application of two rounds of pressure cycling previously resulted in the generation of a majority male profile at a 20:1 female epithelial cell to sperm cell ratio with a male DNA contribution of $88 \pm 0.77 \%$. At 50:1, 100:1, and 200:1, selectivity was not as high with male DNA contributions of $67 \pm 2.0 \%$ and $59 \pm 6.0 \%$ and $43 \pm 13 \%$ respectively. Samples at these concentrations were tested with an immunomagnetic capture (IMC) pretreatment. All three cases resulted in increased selectivity as displayed in Figure 57.

At 50:1 a male DNA contribution of $91 \pm 0.46 \%$ was achieved. At 100:1 and 200:1, the result was a male contribution of $84 \pm 2.2 \%$ and $79 \pm 4.3 \%$.

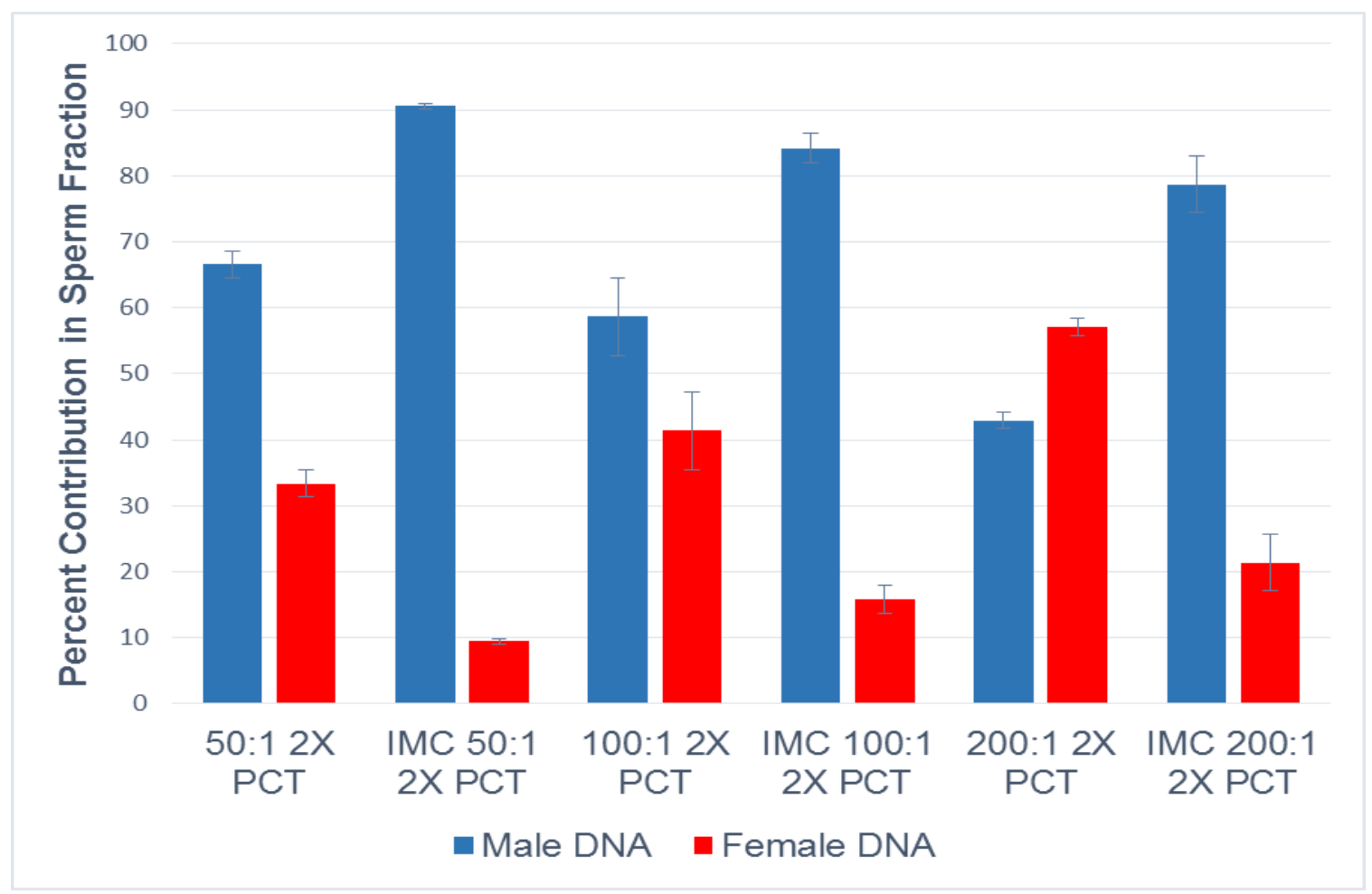

Figure 57. The effect of IMC pretreatment compared to original results with no treatment. Majority male profiles were obtained at every concentration with the addition of IMC 
treatment. Determined by male or female peak height divided by total peak height at 7 loci where no alleles are shared between the male or female contributor, $x 100$. $(n=3 \pm$ standard error)

A majority male profile is observed in the STR profile for each cell ratio. Figures 58 and 59 display STR profiles generated with PowerPlex ${ }^{\circledR} 16 \mathrm{HS}$ of the female and male contributor, respectively, as a reference. The yellow panel for each STR profile generated with PowerPlex ${ }^{\circledR} 16$ HS is displayed in Figures $60-62$.

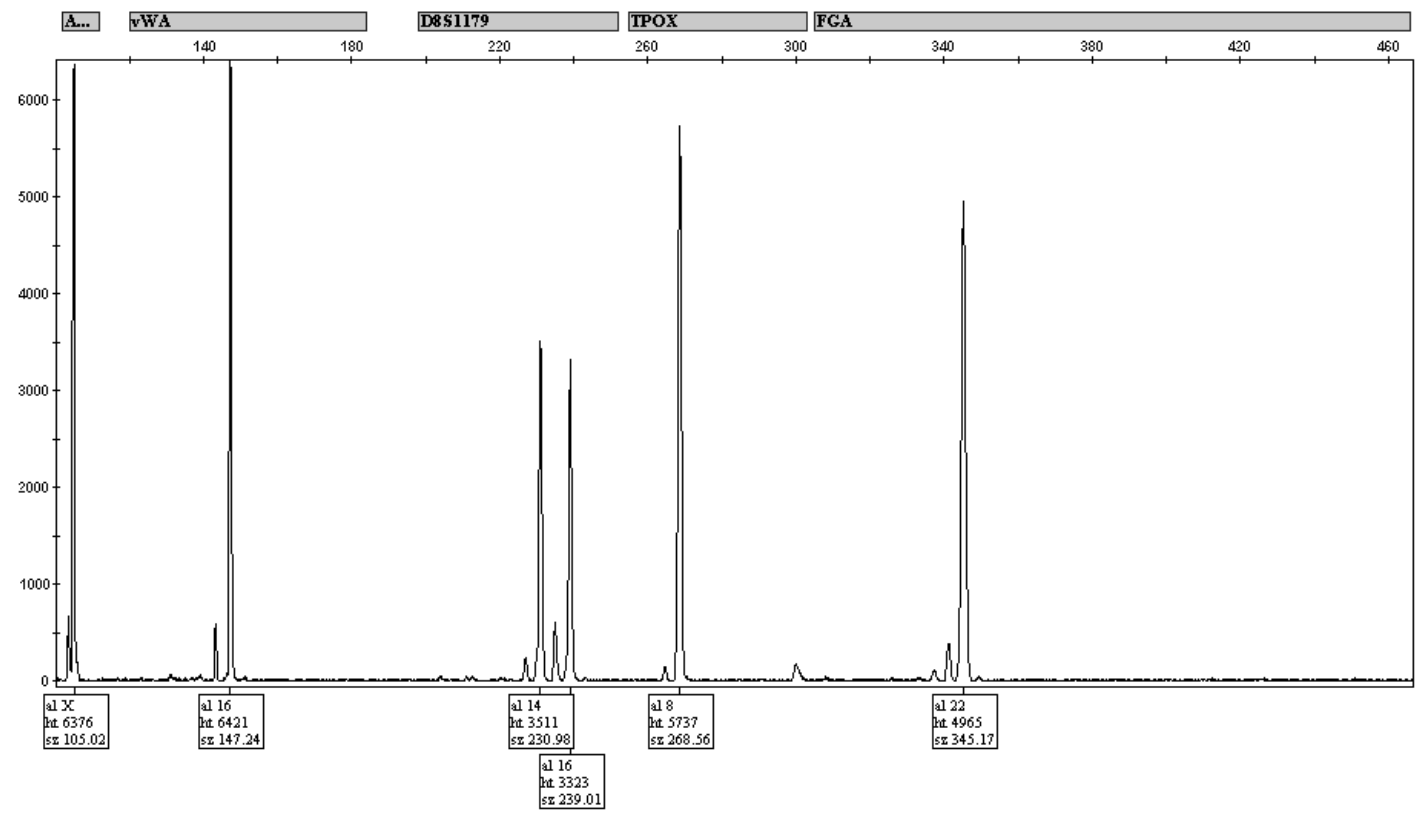

Figure 58. Profile of the female contributor. Panel represents loci labeled with 5-TAMRA (5-Carboxytetramethylrhodamine); Amelogenin, D8S1179, TPOX, and FGA. 


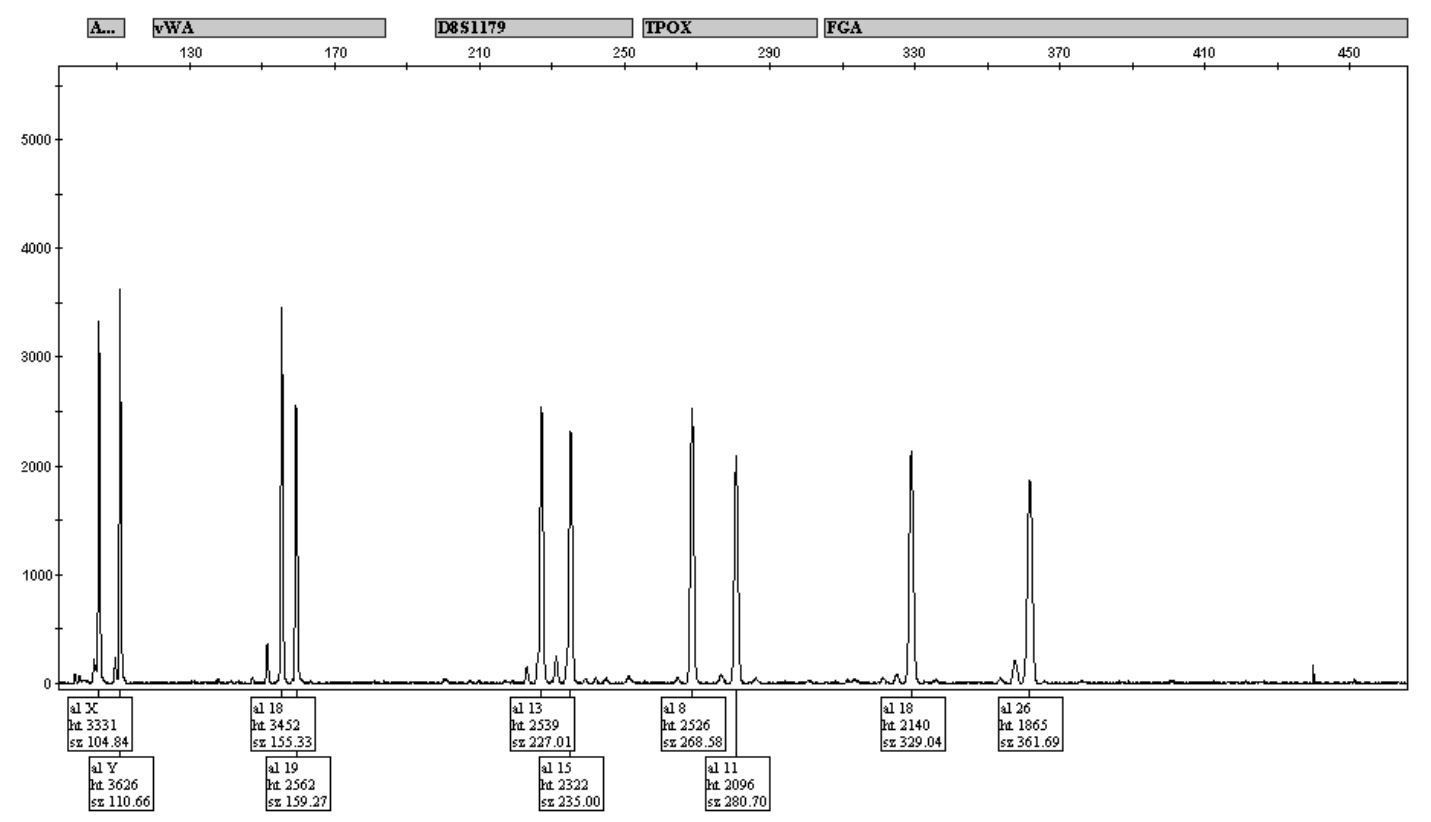

Figure 59. Profile of the male contributor. Panel represents loci labeled with 5-TAMRA (5Carboxytetramethylrhodamine); Amelogenin, D8S1179, TPOX, and FGA.

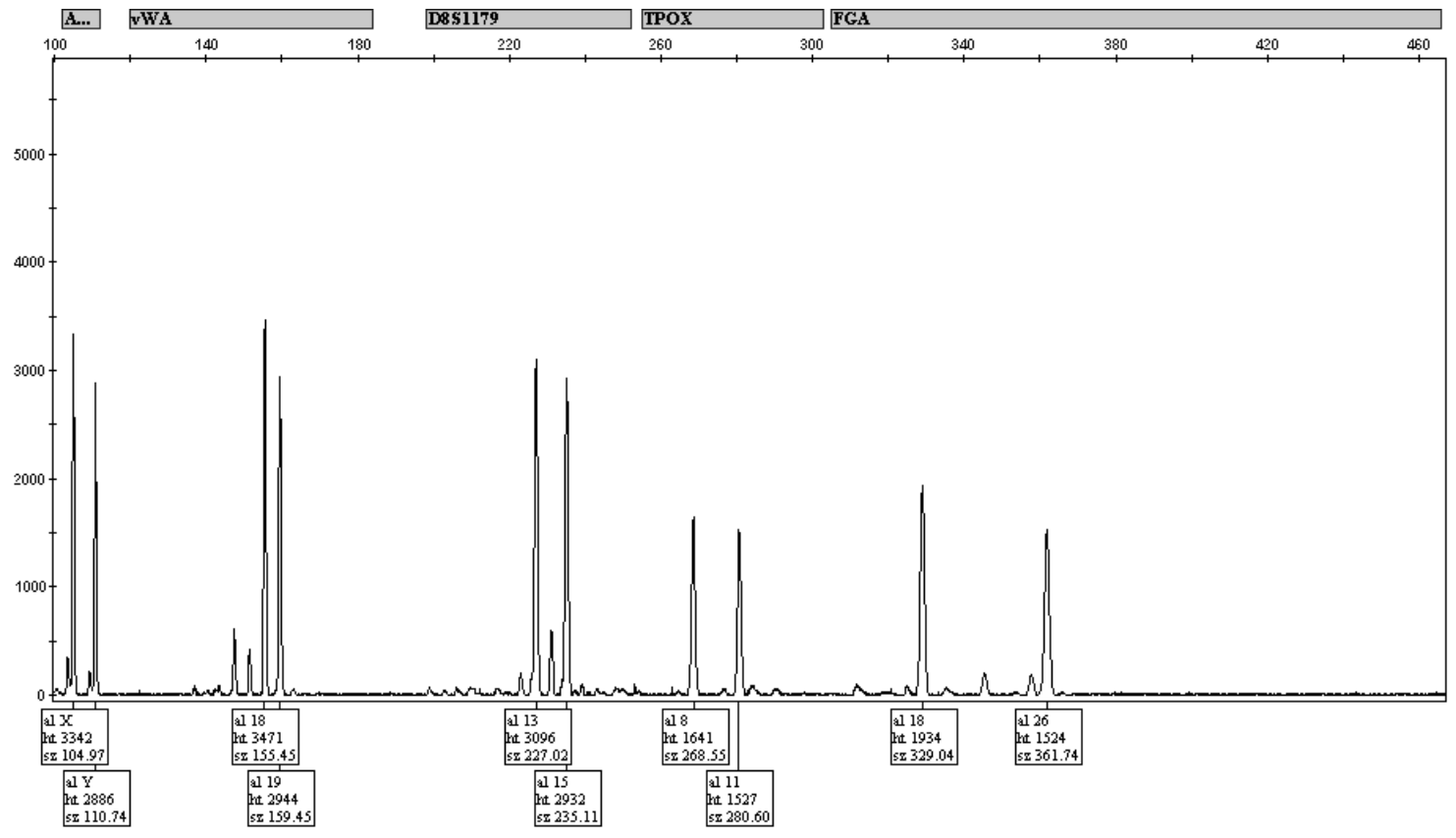

Figure 60. Sperm fraction profile of a 50:1 F:M sample extracted with the modified PCT + alkaline lysis protocol with IMC pretreatment. A percent male DNA contribution of $91 \%$ is observed in the profile. Panel represents loci labeled with 5-TAMRA (5Carboxytetramethylrhodamine); Amelogenin, D8S1179, TPOX, and FGA. 


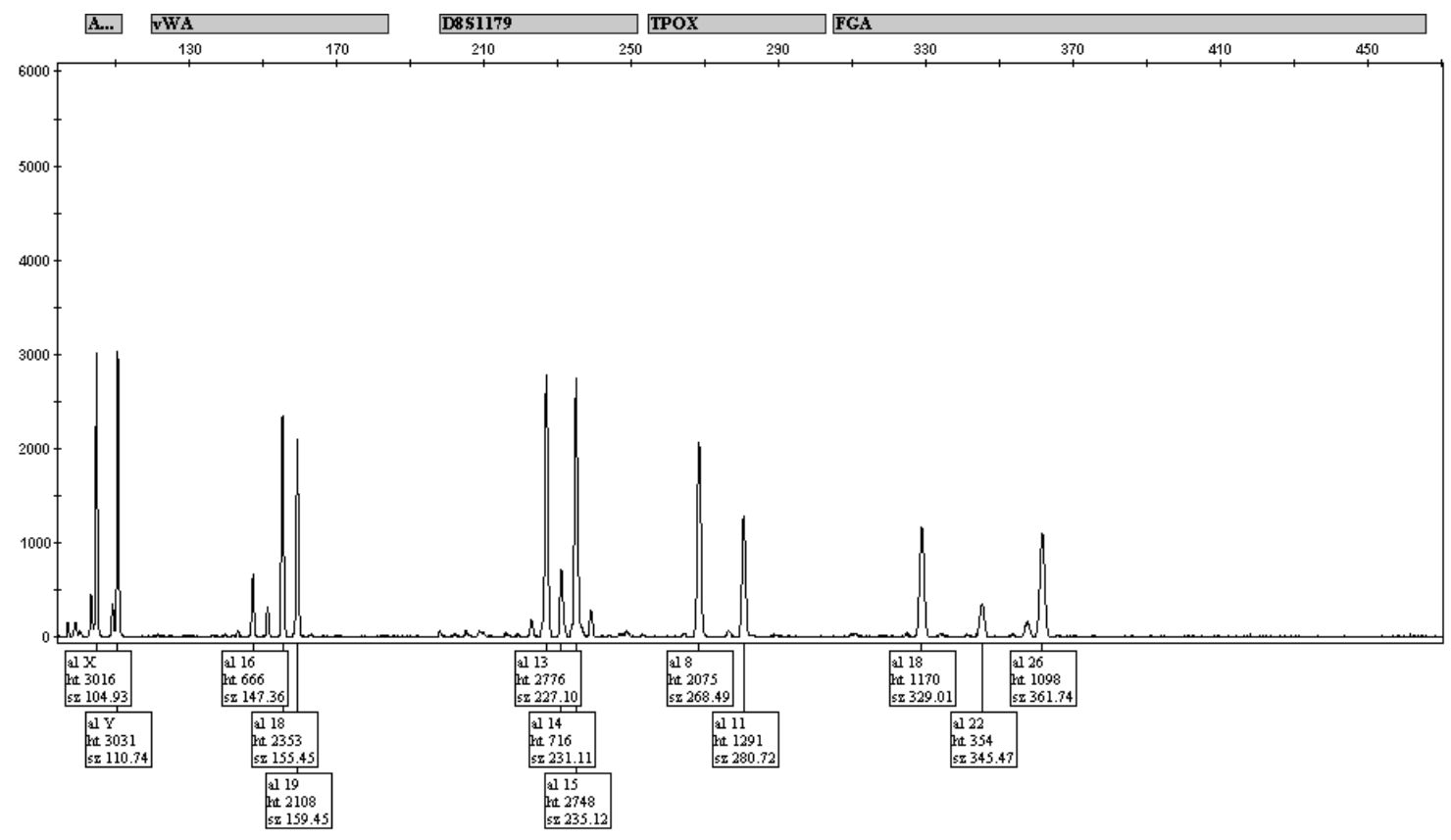

Figure 61. Sperm fraction profile of a 100:1 F:M sample extracted with the modified PCT + alkaline lysis protocol with IMC pretreatment. A percent male DNA contribution of $86 \%$ is observed in the profile. Panel represents loci labeled with 5-TAMRA (5Carboxytetramethylrhodamine); Amelogenin, D8S1179, TPOX, and FGA. 


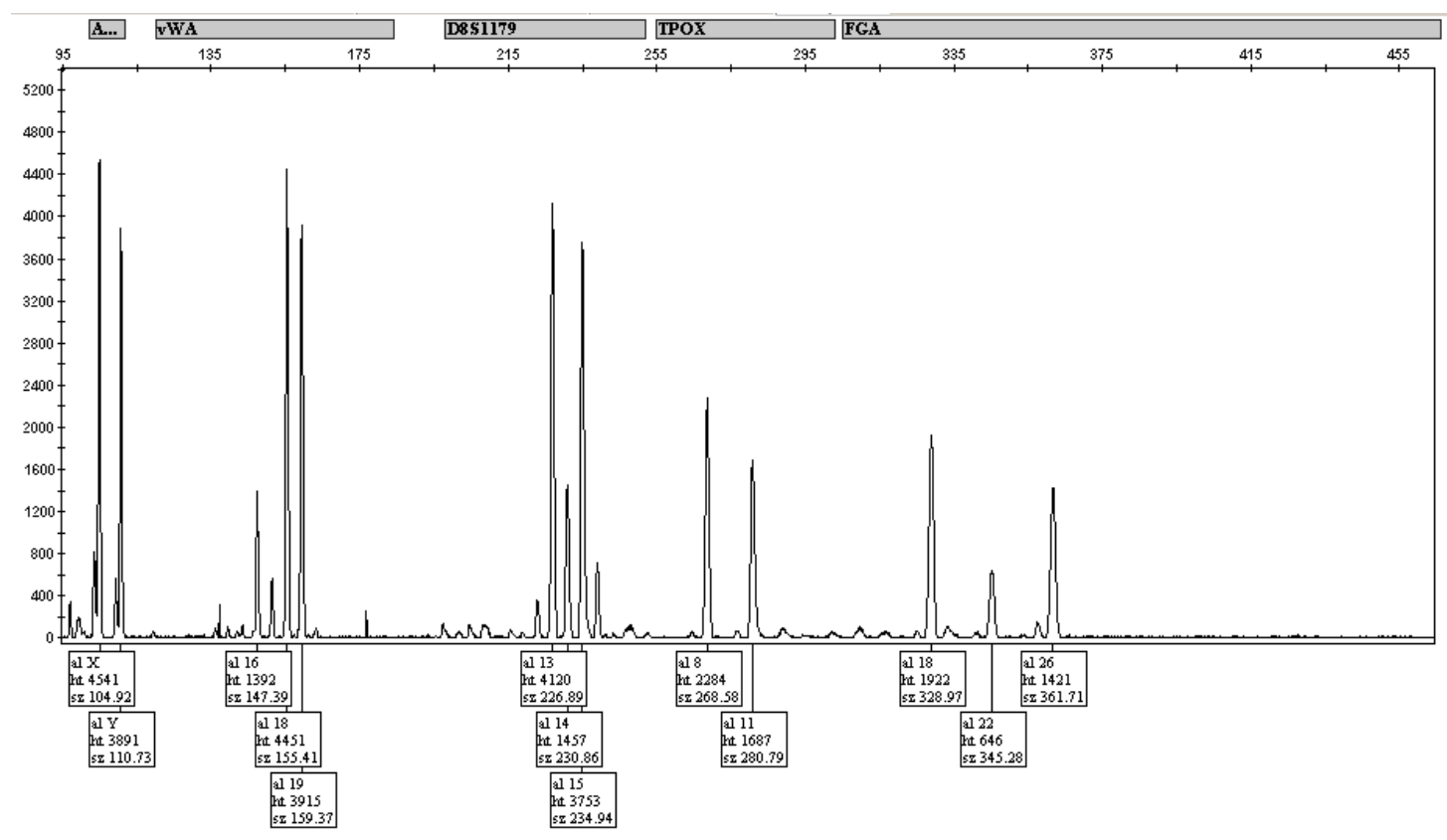

Figure 62. Figure 5. Sperm fraction profile of a 200:1 F:M sample extracted with the modified PCT + alkaline lysis protocol with IMC pretreatment. A percent male DNA contribution of $82 \%$ is observed in the profile. Panel represents loci labeled with 5-TAMRA (5- Carboxytetramethylrhodamine); Amelogenin, D8S1179, TPOX, and FGA.

\section{C.2. Captured Epithelial Cells}

The captured epithelial cell fractions were saved for extraction following the IMC treatment. The results in Figure 63 indicate the removal of a large number of epithelial cells prior to extraction. The captured fractions of the 50:1 samples contained an average of $0.21 \pm 0.017 \mathrm{ng} / \mu \mathrm{L}$. At 100:1 the result was an average of $0.33 \pm 0.038$ $\mathrm{ng} / \mu \mathrm{L}$. A much larger average concentration was found in the 200:1 samples; 0.80 \pm 0.12 $\mathrm{ng} / \mu \mathrm{L}$. These results indicate that only $1.3-1.9 \%$ of female DNA added to sample was captured, but considering that only $0.60 \%-0.76 \%$ female DNA is left behind in the swab without pretreatment that small amount is enough to make a significant difference. Male DNA detected in the captured fractions was negligible with an average of 
$0.000326 \pm 0.000121 \mathrm{ng} / \mu \mathrm{L}(\mathrm{n}=9 \pm$ standard error $)$ and $0.0006 \mathrm{ng} / \mu \mathrm{L}$ at the highest. This small amount of male DNA may originate from male epithelial cells or lysed sperm.

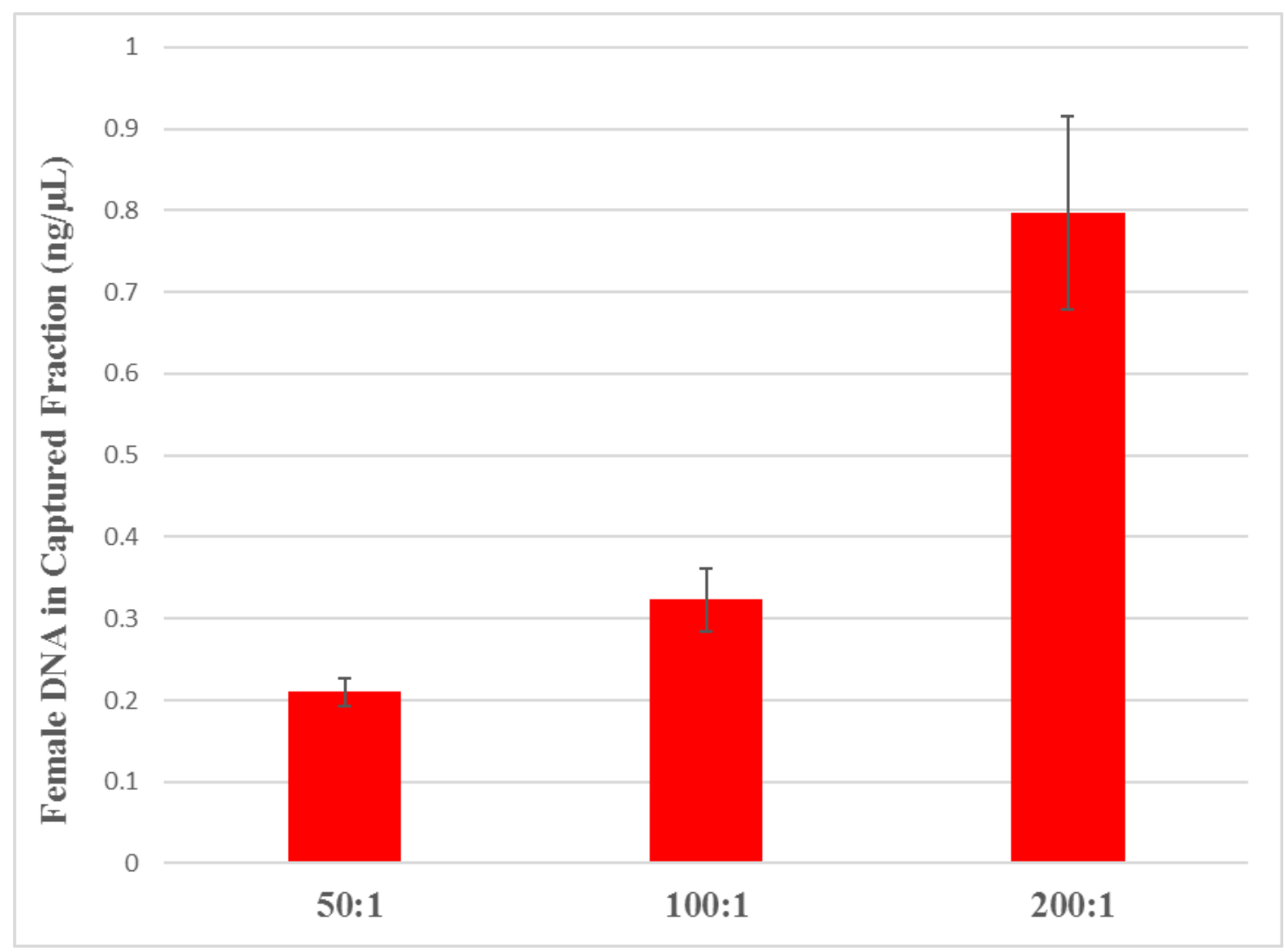

Figure 63. Female DNA recovered in the captured fraction following IMC pretreatment. The greatest number of epithelial cells were predictably captured at 200:1. ( $n=3 \pm$ standard error)

\section{C.3. Stability test}

As backlog samples may be maintained in storage for many years. A post-coital sample collected in 2011 and stored for 5 years at $-80^{\circ} \mathrm{C}$ was extracted with IMC pretreatment in order to determine its applicability for backlog reduction purposes. Selfreported data collected from the volunteer indicated collection took place 13-18 hours after intercourse and 6-14 days since menstruation. The volunteer was reported to be 2130 years of age with a partner that had not been vasectomized. The results are displayed 
in Figure 64. Application of the IMC pretreatment procedure resulted in a percent male DNA contribution of $39 \%$. Investigation of the captured fraction and supernatant reveals important information. Results are depicted in Figure 65. The concentration in the captured fraction was determined to be $24.1 \mathrm{ng} / \mu \mathrm{L}$. An additional $43.2 \mathrm{ng} / \mu \mathrm{L}$ was left behind in the supernatant. It is possible that the high number of epithelial cells saturated available antibodies leading to incomplete separation.

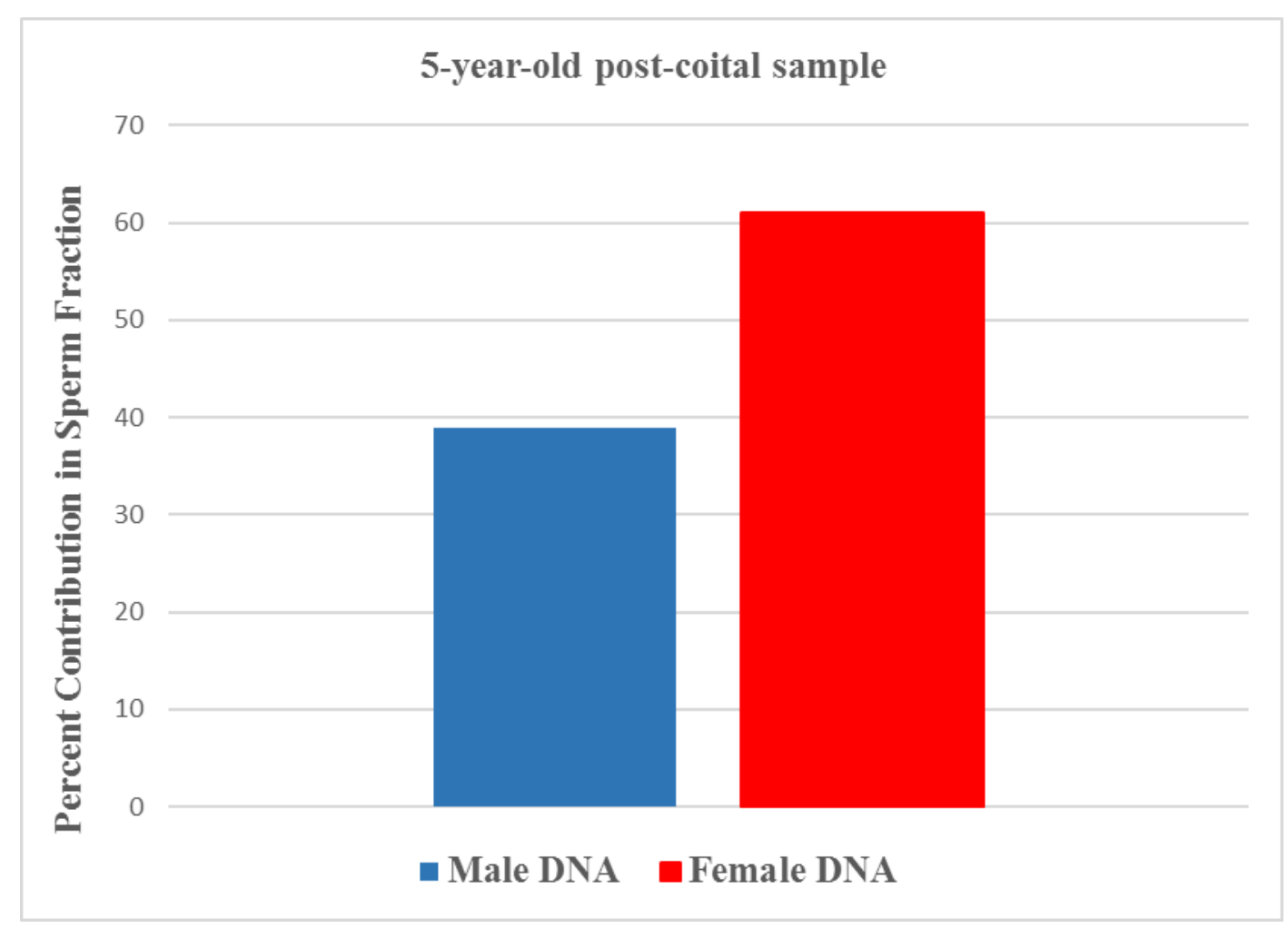

Figure 64. Stability of the method was tested with a 5-year-old post-coital sample. A male DNA contribution of $39 \%$ was obtained. Determined by male or female peak height divided by total peak height at 7 loci where no alleles are shared between the male or female contributor. 


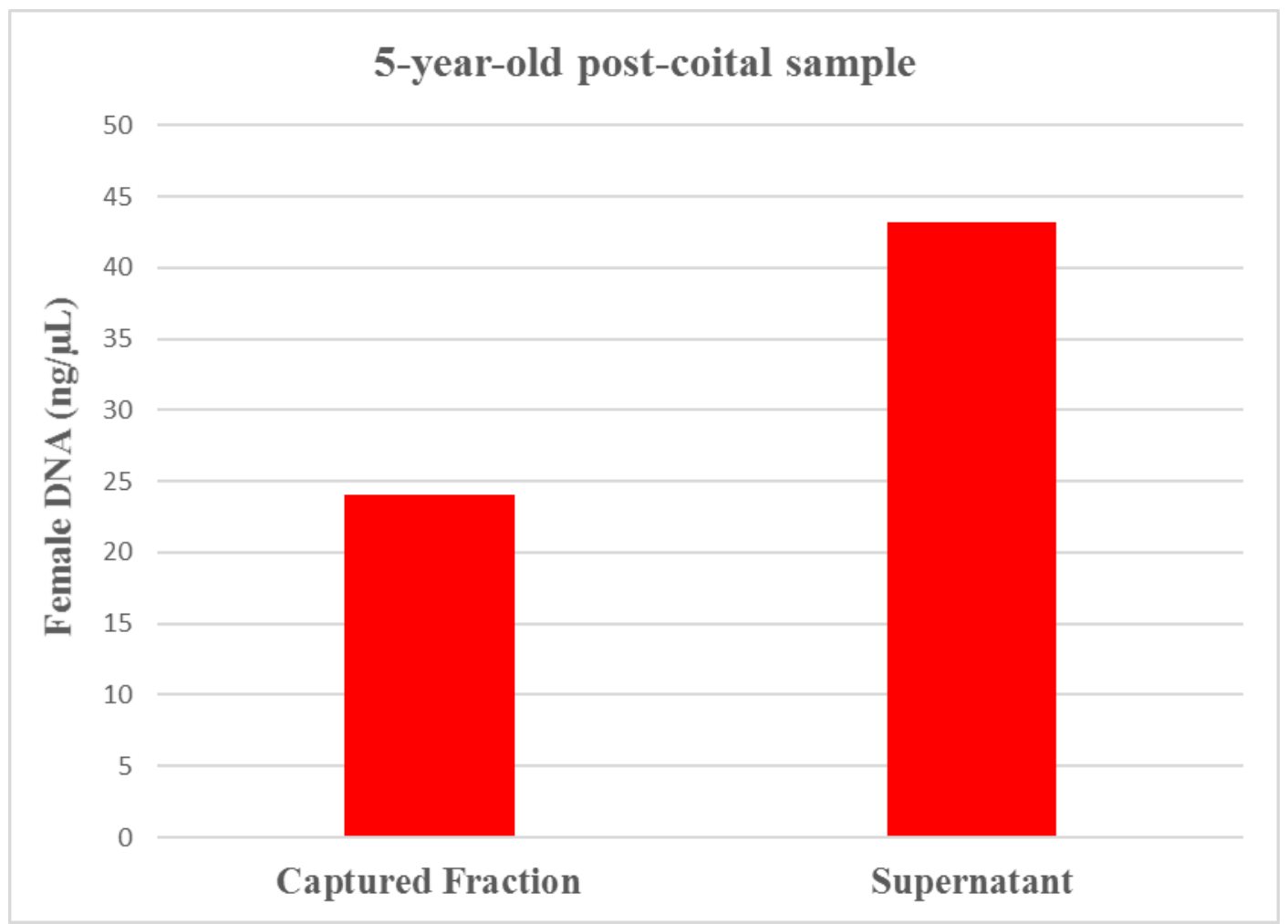

Figure 65. Female DNA recovered in the captured fraction and supernatant following IMC pretreatment of a 5-year-old post-coital sample. A concentration of $24.1 \mathrm{ng} / \mu \mathrm{L}$ was detected in the captured fraction with $43.2 \mathrm{ng} / \mu \mathrm{L}$ left behind in the supernatant.

\section{Concluding Remarks}

Immunomagnetic capture of epithelial cells has the potential to reduce the number of epithelial cells in a sample prior to extraction with a negligible loss of sperm cell DNA. A significant number of epithelial cells were still captured from a 5-year-old post-coital swab demonstrating the capability of the procedure even with older samples. The high concentration of cells found in the captured fraction for the post-coital sample and in the supernatant indicated a possible saturation of the added antibodies. Additional reagent may improve results. In casework, swab cuttings are used rather than entire swab heads. This would reduce the number of epithelial cells present in the sample and reduce the amount of reagent needed for efficient capture and separation. 


\section{CHAPTER IX. CONCLUSIONS}

Sexual assault evidence samples are among the most challenging encountered in forensic laboratories. Such samples are often overwhelmed by the presence of the victim's epithelial cells. In order to obtain a clear suspect profile, it is necessary to separate these epithelial cells from the suspect's sperm cells collected in the sample.

The developed two-step protocol utilizes the Barocylcer ${ }^{\circledR}$ NEP 2320 from Pressure BioSciences, Inc. to selectively lyse epithelial cells in alkaline conditions. This is followed by alkaline lysis and high temperature extraction of sperm cells. This technique has resulted in high recovery of sperm cell DNA from cotton swabs, $69 \pm 6 \%$, and clear male profiles at low epithelial cell to sperm cell ratios. At higher ratios, selectivity decreases and mixed or majority female profiles are observed [75].

This study was conducted to improve the selectivity of this method at higher female epithelial cell to sperm cell ratios. An increase in epithelial cell recovery occurred at $0.2 \mathrm{~N} \mathrm{NaOH}$ compared to $0.4 \mathrm{NaOH}$. [75]. This led to experiments at lower concentrations during the PCT step to test for further improvement. Increased epithelial cell DNA recovery and decreased sperm cell disruption at $0.05 \mathrm{~N} \mathrm{NaOH}$ resulted in improved selectivity at 20:1 female epithelial cell to sperm cell ratio with a male DNA contribution of $71 \pm 1.6 \%$ compared with $18 \pm 0.92 \%$ obtained with the original protocol using $0.4 \mathrm{~N} \mathrm{NaOH}$ for both cell lysis steps.

Experiments varying temperatures during the PCT step revealed no improvement with either increasing or decreasing temperature when compared to room temperature. 
Epithelial cell fraction quantitation data suggested these results are due to male DNA loss at higher temperatures and lower epithelial cell DNA recovery at lower temperatures.

The inclusion of an additional pressure cycling step allowed for further recovery of epithelial cell DNA left behind in the swab and the successful generation of nearly clear male profiles at 20:1 female epithelial cell to sperm cell ratios with a male DNA contribution of $88 \pm 0.77 \%$. This treatment also resulted in majority male profiles at 50:1 and $100: 1,67 \pm 2.0 \%$ and $59 \pm 6.0 \%$.

Validation studies were performed to determine the effectiveness of the modified protocol with various sample types and conditions. Reproducibility studies showed variation over the course of three day in recovery, but consistent results for selectivity.

The correlation study revealed greater recovery for several post-coital swab sample cuttings samples with the PCT and alkaline lysis protocol compared to the established PBSO differential extraction method, but selectivity was not as high with most samples. One sample was tested in triplicate with both methods. Results coincided with those of the ten individual samples; higher recovery and lower selectivity. Higher reproducibility was also achieved with the PCT and alkaline lysis protocol.

Case-type samples included epithelial cell and sperm cell suspensions deposited on cuttings from denim jeans, cotton panties, and a cotton/polyester bedspread. Recovery was extremely low with samples deposited on the bedspread, but selectivity was high as only male alleles were detected in bedspread samples. This may be due to the low absorbance of the cotton/polyester material. Epithelial cell DNA was likely easily 
removed from the substrate along with the majority of sperm cells. One round of pressure cycling may be sufficient for non-absorbent substrates.

Both recovery and selectivity decreased compared to cotton swab controls with samples deposited on denim and cotton panty fabric cuttings. The results for the postcoital samples, denim, and cotton panty cuttings may be due to the large volumes of $\mathrm{NaOH}$ used for the PCT step; $800 \mu \mathrm{L}$. As this protocol was originally developed for the extraction of entire swabs, this volume may lead to inefficient transmittance of pressure to smaller substrates such as cuttings from swab or fabric. Low volume sample tubes manufactured for the Barocycler instrument may also be of interest for such samples.

Stability studies were performed with samples subjected to environmental insults and with the addition of inhibitors; bile salts and tannic acid. Results showed little loss in selectivity or recovery due to the addition of bile salts or tannic acid.

Exposure to outdoor conditions for one week only resulted in a minor decrease in male DNA contribution in the sperm fraction compared to controls, $58 \pm 1.7 \%$ to $52 \pm 3.1 \%$. Male DNA recovery remained within range of control results.

According to sensitivity studies, male alleles were visible with as few as 75 sperm cells present in the sample. At a 5:1 epithelial cell to sperm cell ratio, male alleles were detected by the GeneMapper ${ }^{\circledR}$ software at 14 of the 16 loci. Unfortunately, sensitivity studies also revealed decreasing selectivity with decreasing cell count. This may be due to incomplete sperm cell recovery from the swab. Studies also revealed rather reproducible selectivity results for 3 sets of samples over the course of 3 days, but lower reproducibility in male DNA recovery. 
Immunomagnetic capture was successfully applied to swab samples with epithelial cell to sperm cell ratios as high as 200:1 to obtain nearly clear male profiles. Epithelial cells were successfully captured from a post-coital sample stored at $-80^{\circ} \mathrm{C}$ for 5 years thus demonstrating the stability of the technique.

Overall, these results present pressure cycling with immunocapture as a promising new extraction method that is capable of high recovery as well as high selectivity in certain conditions with no wash steps and short processing times. Work must continue to improve the effectiveness of this technique with various substrates and at various samples concentrations. Immunomagnetic capture of epithelial cells has shown great potential as a pretreatment for samples overwhelmed with epithelial cells pertaining to the victim. The technique can be applied to improve the selectivity of any extraction method.

\section{CHAPTER X. FUTURE WORK}

Experiments must continue to further improve this protocol and solve issues with samples containing low amounts of DNA and or deposited on different substrates.

Studies should be done to test the effectiveness of the protocol with samples deposited on swab and fabric cuttings with the use lower volumes of $\mathrm{NaOH}$ during the PCT step. It is possible that selectivity of the protocol suffered with low DNA samples due to incomplete recovery of male DNA from the swab. The effect of adding non-human DNA as a carrier should be examined for its potential to assist the recovery of DNA from such low level samples. Carriers minimize DNA loss on walls of tubes and to substrates by competing with nucleic acids present in the sample for binding sites [120]. 
The immunomagnetic cell capture technique should continue to be tested with cuttings from vaginal swabs to determine the optimal amount of reagent needed for efficient separation with more realistic samples. Studies should also continue with the remaining post-coital swabs to further substantiate the effectiveness of IMC pretreatment with samples kept in storage for several years. Sample aged for days or weeks could also be used to track the stability of the technique over time.

The presence of magnetic particles left behind in the swab following immunomagnetic cell capture treatment interfered with purification by Biorobot EZ1. This necessitated the use of Phenol/Chloroform/Isoamyl Alcohol (25:24:1) (PCIA) purification and ethanol precipitation. Methods should be investigated to remove these magnetic particles from the lysate for simplified processing. 


\section{LIST OF REFERENCES}

1. Abaz, J., Walsh S. J., Curran J. M., Moss D. S., Cullen J., Bright J. A., Power, T. E. Comparison of the variables affecting the recovery of DNA from common drinking containers. Forensic Science International 2002, 126, 233-240.

2. Aboud, M. (2012). The development of direct ultra-fast PCR for forensic genotyping using short channel pinchedidic systems with enhanced sieving matrices. Ph.D. Dissertation, Florida International University, Miami FL, 2012.

3. Aditya, S., Sharma, A. K., Bhattacharyya, C. N., \& Chaudhuri, K. Generating STR profile from "touch DNA." Journal of Forensic and Legal Medicine, 2011, 18(7), 295-298.

4. Alaeddini R. Forensic implications of PCR inhibition--A review. Forensic Science International: Genetics 2012, 6 (3), 297-305.

5. Alberts B. Molecular Biology of the Cell: Reference Edition. Garland Science: 2008

6. Anslinger, K., Bayer, B., Danilov, S. M. \& Metzger, R. Application of spermspecific antibodies for the separation of sperm from cell mixtures. Forensic Sci. Int. Genet. Suppl. Ser. 2008, 1, 394-395 (2008).

7. Atsü S. S., Gökdemir K., Kedici P. S., Ikyaz, Y. Y. (1998). Bitemarks in forensic odontology. The Journal of Forensic Odonto-stomatology 1998, 16(2), 30-34.

8. Bai, R., Liu, Y., Li, Z., Jin, H., Tian, Q., Shi, M., \& Ma, S. Developmental Validation of a novel 5 dye Y-STR System comprising the 27 YfilerPlus loci. Scientific Reports. 2016, 6, 29557.

9. Baird, J. B. The Individuality of Blood and Bloodstains: A Non-Critical Historical Review with Some Reference to the More Recent Canadian Endeavours to Characterize Human Blood. Canadian Society of Forensic Science Journal 1978, 11 (2), 83-122.

10. Barni F., Lewis,S. W., Berti A., Miskelly G. M., Lago, G. Forensic application of the luminol reaction as a presumptive test for latent blood detection. Talanta 2007, 72 (3), 896-913.

11. Berg, J.M., Tymoczko, J.L., Stryer, L. Biochemistry $6^{\text {th }}$ edition. W.H. Freeman and Company: 2007

12. Biega L.A., Duceman, B.W. Substitution of $\mathrm{H}_{2} \mathrm{O}$ for formamide in the sample preparation protocol for STR analysis using the capillary electrophoresis system: the effects on precision, resolution, and capillary life. Journal of Forensic Sciences 1999, 44, 1029-1031. 
13. Birky Jr. C. W. The inheritance of genes in mitochondria and chloroplasts: laws, mechanisms, and models. Annual Review of Genetics 2001, 35 (1), 125-148.

14. Birnboim H. C., Doly J. A rapid alkaline extraction procedure for screening recombinant plasmid DNA. Nucleic Acids Research 1979, 7, 1513-23.

15. Blears M.J., De Grandis S.A., Lee H., Trevors J.T. Amplified fragment length polymorphism (AFLP): a review of the procedure and its applications. Journal of Industrial Microbiology and Biotechnology 1998, 21 (3), 99-114.

16. Bright, J., Veth, J. S., Vintiner, S. K., Cockerton, S. L., Curnow, N., Dalzell, J. A., \& Meredith, M. L. Laser microdissection methodology in forensic casework. 2012, 44(2), 135-144.

17. Brownlow R. J., Dagnall K. E., Ames C. E. A comparison of DNA collection and retrieval from two swab types (cotton and nylon flocked swab) when processed using three QIAGEN extraction methods. Journal of Forensic Sciences 2012, 57 (3), 713-717.

18. Bruner E.A., Okubara P.A., Abi-Ghanem R., Brown D.J., Reardon C.L. Use of pressure cycling technology for cell lysis and recovery of bacterial and fungal communities from soil. Biotechniques. 2015, 58(4):171-80.

19. Bessetti, J. An introduction to inhibitors. Profiles in DNA 2007, 10(1), 9-10

20. Budowle B., Bieber F. R., Eisenberg A. J. Forensic aspects of mass disasters: strategic considerations for DNA-based human identification. Legal Medicine 2005, 7(4), 230-243.

21. Buel E., Schwartz M. B., LaFountain M. J. Capillary electrophoresis STR analysis: Comparison to gel-based systems. Journal of Forensic Sciences 1998, 43, 164-170.

22. Butler J. M., Advanced Topics in Forensic DNA Typing: Methodology. Elsevier Science \& Technology, 2011.

23. Butler J. M., Buel E., Crivellente F., McCord B. R., Forensic DNA typing by capillary electrophoresis using the ABI Prism 310 and 3100 genetic analyzers for STR analysis. Electrophoresis 2004, 25 (10-11), 1397-412.

24. Butler J. M., Forensic DNA Typing: Biology, Technology, and Genetics of Str Markers. Elsevier Academic Press, 2005.

25. Butler J. M., Genetics and genomics of core short tandem repeat loci used in human identity testing. Journal of Forensic Sciences 2006, 51 (2), 253-65.

26. Byrdsong, C. Evaluation of manual differential separation in comparison to QIAcube automation 2014 
27. Carson, C., Dr. Garvin, A., Gorman, K. Automated Processing of Sexual Assault Cases Using Selective Degradation. U.S. Department of Justice 2013

28. Comey C. T. DNA extraction strategies for amplified fragment length polymorphism analysis. Journal of Forensic Sciences 1994, 39 (5): 1254-69.

29. Delidow B. C., Lynch J. P., Peluso J. J., White, B. A. Polymerase chain reaction. In PCR Protocols Humana Press. 1993, 1-29.

30. Demeke T., Jenkins G. R. Influence of DNA extraction methods, PCR inhibitors and quantification methods on real-time PCR assay of biotechnology-derived traits. Analytical and Bioanalytical Chemistry 2010, 396 (6), 1977-1990.

31. Frégeau C. J., De Moors A. Competition for DNA binding sites using Promega DNA IQ ${ }^{\mathrm{TM}}$ paramagnetic beads. Forensic Science International: Genetics 2012, 6 (5), 511-522.

32. Garrett P.E., Tao F., Lawrence N., Ji J., Schumacher R.T., Manak M.M. Tired of the same old grind in the new genomics and proteomics era? TARGETS 2002, 1, 156162.

33. Gill, P., Jeffreys, A. J., \& Werrett, D. J. Forensic application of DNA

“fingerprints". Nature, 1985, 318(6046), 577-579.

34. Goodwin, W., Linacre, A., \& Hadi, S. (2011). An Introduction to Forensic Genetics. Journal of Chemical Information and Modeling (Vol. 53).

35. Goodwin, W., Linacre, A., Hadi, S. An Introduction to Forensic, $2^{\text {nd }}$ Edition. Wiley: 2010

36. Gossett, D. R., Weaver, W. M., MacH, A. J., Hur, S. C., Tse, H. T. K., Lee, W., ... Di Carlo, D. Label-free cell separation and sorting in microfluidic systems. Analytical and Bioanalytical Chemistry, 2010, 397(8), 3249-3267.

37. Baker, D.R., Capillary Electrophoresis. Wiley-Interscience: 1995.

38. Greenspoon S.A., Scarpetta M.A., Drayton M.L., Turek S.A. QIAamp spin columns as a method of DNA isolation for forensic casework. Journal of Forensic Sciences 1998, 43, 1024-30.

39. Gross V., Carlson G., Kwan A.T., Smejkal G., Freeman E., Ivanov A.R., Lazarev A. Tissue fractionation by hydrostatic pressure cycling technology: the unified sample preparation technique for systems biology studies. Journal of Biomolecular Techniques 2008, 19, 189-199. 
40. Hanson, E., Haas, C., Jucker, R., \& Ballantyne, J. Specific and sensitive mRNA biomarkers for the identification of skin in "touch DNA" evidence. Forensic Science International: Genetics, 2012, 6(5), 548-558.

41. Hares, D.R. Selection and implementation of expanded CODIS core loci in the United States. Forensic Sci. Int. Genet. 2015. 17:33-34.

42. Hatsch, D. et al. High-throughput mitochondrial DNA cloning in forensic and anthropological studies. Int. Congr. Ser. 2006, 1288, 118-120.

43. Hebda, L. M., Doran, A. E., \& Foran, D. R. Collecting and analyzing DNA evidence from fingernails: A comparative study. Journal of Forensic Sciences, 2014, 59(5), 1343-1350.

44. Hochmeister M. N., Budowle B., Rudin O., Gehrig C., Borer U., Thali M., Dirnhofer, R. Evaluation of prostate-specific antigen (PSA) membrane test assays for the forensic identification of seminal fluid. Journal of Forensic Sciences 1999, 44, $1057-1060$.

45. Hoff-Olsen P., Mevåg B., Staalstrøm E., Hovde B., Egeland T., Olaisen B. Extraction of DNA from decomposed human tissue: an evaluation of five extraction methods for short tandem repeat typing. Forensic Science International 1999, 105 (3), 171-183.

46. Holland P. M., Abramson R. D., Watson R., Gelfand D. H. Detection of specific polymerase chain reaction product by utilizing the 5 '----3'exonuclease activity of Thermus aquaticus DNA polymerase. Proceedings of the National Academy of Sciences 1991, 88 (16), 7276-7280.

47. Horsman K. M., Barker S. L. R., Ferrance J. P., Forrest K. A., Koen K. A., Landers J. P. Separation of sperm and epithelial cells in a microfabricated device: Potential application to forensic analysis of sexual assault evidence. Analytical Chemistry 2005, 77 (3), $742-749$.

48. Houk, M.M., Siegel, J.A. Fundamentals of Forensic Science, Second Edition. Elsevier: 2010

49. Hudlow W. R., Buoncristiani M. R. Development of a rapid, 96-well alkaline based differential DNA extraction method for sexual assault evidence. Forensic Science International Genetics 2012, 6 (1), 1-16.

50. Innis, M. A.; Sninsky, J. J.; Gelfand, D. H., Pcr Applications: Protocols for Functional Genomics. Academic Press: 1999; pp 33-47.

51. James, S.H., Nordby, J.J., Bell, S. Forensic Science: An Introduction to Scientific and Investigative Techniques $4^{\text {th }}$ edition. CRC Press Taylor and Francis Group: 2014 
52. Jeffreys A.J., Wilson V., Thein S.L. Individual- specific fingerprints of human DNA. Nature. 1985, 316, 76-9.

53. Kipps A. E., Whitehead P. H. The significance of amylase in forensic investigations of body fluids. Forensic Science 1975, 6(3), 137-144.

54. Klintschar M, Neuhuber F. Evaluation of an alkaline lysis method for the extraction of DNA from whole blood and forensic stains for STR analysis. Journal of Forensic Sciences 2000, 45 (3), 669-673.

55. Krenke, B. E., Nassif, N., Sprecher, C. J., Knox, C., Schwandt, M., \& Storts, D. R. Developmental validation of a real-time PCR assay for the simultaneous quantification of total human and male DNA. Forensic Science International: Genetics 2008, 3 (1), 14-21.

56. Lawless, C. J. The low template DNA profiling controversy: Biolegality and boundary work among forensic scientists. Social Studies of Science, 2012, 43(2), 191214.

57. Lee H. C., Ladd C. Preservation and collection of biological evidence. Croatian Medical Journal 2001, 42 (3), 225-228.

58. Lee H. C., Palmbach T. M., Primorac D., Andelinovic S. Collection and Preservation of Physical Evidence. Forensic DNA Applications: An Interdisciplinary Perspective 2014, 253.

59. Lewin, B. Genes IX. Jones and Bartlett Publishers.2008

60. Li, X., Wang, Q., Feng, Y. et al. Magnetic bead-based separation of sperm from buccal epithelial cells using a monoclonal antibody against MOSPD3. International Journal of Legal Medicine. 2014, 128: 905.

61. Lincoln C. A., McBride P. M., Turbett G. R., Garbin C. D., MacDonald E. J. The use of an alternative light source to detect semen in clinical forensic medical practice. Journal of Clinical Forensic Medicine 2006, 13 (4), 215-218.

62. Liu, W., Chen, W., Liu, R., Ou, Y., \& Liu, H. Separation of sperm and epithelial cells based on the hydrodynamic effect for forensic analysis. 2015, 44127, 1-14.

63. Lounsbury J. A., Coult N., Miranian D. C., Cronk S. M., Haverstick D. M., Kinnon P., Landers J. P. An enzyme-based DNA preparation method for application to forensic biological samples and degraded stains. Forensic Science International: Genetics 2012, $6(5), 607-615$.

64. Manual EasySep ${ }^{\circledR}$ Protocol Using Purple EasySep ${ }^{\circledR}$ Magnet (Catalog \#18000), Stemcell Technologies, 2009, 1-6 
65. Marmur J. A procedure for the isolation of deoxyribonucleic acid from microorganisms. Journal of Molecular Biology 1961, 3 (2), 208-218.

66. Marshall, P. L., King, J. L., Lawrence, N. P., Lazarev, A., Gross, V. S., \& Budowle, B. Pressure cycling technology (PCT) reduces effects of inhibitors of the PCR. International Journal of Legal Medicine. 2013, 127(2), 321-333.

67. McClintock J. T. Forensic Analysis of Biological Evidence: A Laboratory Guide for Serological and DNA Typing. CRC Press, 2014

68. McCord, B., Pionzio, A. Thompson, R. Analysis of the Effect of a Variety of PCR Inhibitors on the Amplification of DNA using Real Time PCR . U.S. Department of Justice 2015.

69. McNevin D., Wilson-Wilde L., Robertson J., Kyd J., Lennard C. Short tandem repeat (STR) genotyping of keratinised hair: Part 1. Review of current status and knowledge gaps. Forensic Science International 2005, 153 (2), 237-246.

70. Leong, K. G., Niessen, K., Kulic, I., Raouf, A., Eaves, C., Pollet, I., \& Karsan, A. Jagged1-mediated Notch activation induces epithelial-to-mesenchymal transition through Slug-induced repression of E-cadherin. The Journal of Experimental Medicine 2007, 204(12), 2935-2948.

71. Moss D., Harbison S. A., Saul D. J. An easily automated, closed-tube forensic DNA extraction procedure using a thermostable proteinase. International Journal of Legal Medicine 2003, 117 (6), 340-349.

72. Mullis, K. B. The unusual origin of the polymerase chain reaction. Scientific American. 1990, 262 (4), 56-61.

73. Nagy M., Otremba P., Kruger C., Bergner-Greiner S., Anders P., Henske B., Prinz M., Roewer L. Optimization and validation of a fully automated silica-coated magnetic beads purification technology in forensics. Forensic Science International 2005, 152 (1), 13-22.

74. Nori, D. Differential extraction of DNA from sperm and epithelial cells recovered from sexual assault kits using alkaline lysis and pressure cycling technology (PCT). Ph.D. Dissertation, Florida International University, Miami FL, 2015.

75. Nori, D. V., McCord, B. R. The application of alkaline lysis and pressure cycling technology in the differential extraction of DNA from sperm and epithelial cells recovered from cotton swabs. Anal. Bioanal. Chem. 2015, 407, 6975-6984.

76. Norris J.V., Manning K., Linke S. J., Ferrance J. P., Landers J. P. Expedited, chemically enhanced sperm cell recovery from cotton swabs for rape kit analysis. Journal of Forensic Sciences 2007, 52 (4), 800-805. 
77. Norris, J. V., Evander, M., Horsman-Hall, K. M., Nilsson, J., Laurell, T., \& Landers, J. P. Acoustic differential extraction for forensic analysis of sexual assault evidence. Analytical Chemistry, 2009, 81(15), 6089-6095.

78. Okubara, P. A., Li, C., Schroeder, K. L., Schumacher, R. T., \& Lawrence, N. P. Improved extraction of Rhizoctonia and Pythium DNA from wheat roots and soil samples using pressure cycling technology. Canadian Journal of Plant Pathology. 2007, 29(July), 304-310.

79. Olaisen B., Stenersen M., Mevåg B. Identification by DNA analysis of the victims of the August 1996 Spitsbergen civil aircraft disaster. Nature Genetics 1997, 15(4), 402405.

80. Oliva R. Protamines and male infertility. Human Reproduction Update 2006, 12, 417-435.

81. Olszowy, P. P., Burns, A., \& Ciborowski, P. S. Pressure-assisted sample preparation for proteomic analysis. Analytical Biochemistry.2013, 438(1), 67-72.

82. Opel K. L., Chung D., McCord B. R. A Study of PCR Inhibition Mechanisms Using Real Time PCR. Journal of Forensic Sciences 2010, 55 (1), 25-33.

83. Pang B. C. M., Cheung B. K. K. Double swab technique for collecting touched evidence. Legal Medicine 2007, 9 (4), 181-184.

84. Pickrahn, I., Müller, E., Zahrer, W., Dunkelmann, B., Cemper-Kiesslich, J., Kreindl, G., Neuhuber, F. Yfiler ${ }^{\circledR}$ Plus amplification kit validation and calculation of forensic parameters for two Austrian populations. 2016, Forensic Sci Int Genet., 21:904

85. Plexor ${ }^{\circledR}$ HY system, Technical Manual\# TM299, Promega Corp., Madison, WI).

86. Poon H., Elliott J., Modler J., Fregeau, C. The Use of Hemastix ${ }^{\circledR}$ and the Subsequent Lack of DNA Recovery Using the Promega DNA IQ ${ }^{\mathrm{TM}}$ System. Journal of Forensic Sciences 2009, 54 (6), 1278-1286.

87. PowerPlex ${ }^{\circledR}$ Fusion 6C System, Technical Manual\# TMD045, Promega Corp., Madison, WI.

88. PowerPlex ${ }^{\circledR}$ Fusion System, Technical Manual\# TMD039, Promega Corp., Madison, WI.

89. Pray L. Discovery of DNA structure and function: Watson and Crick. Nature Education 2008, 1 (1), 100.

90. Rekha, B. Evaluation of DNA Extraction Protocol for Detection of Salmonella From Animal Foods. 2014, 14, 4373-4376. 
91. Rudbeck, L. \& Dissing, J. Rapid, simple alkaline extraction of human genomic DNA from whole blood, buccal epithelial cells, semen and forensic stains for PCR. Biotechniques 1998, 25, 588-592.

92. Ruitberg C. M., Reeder D. J., Butler, J. M. STRBase: a short tandem repeat DNA database for the human identity testing community. Nucleic Acids Research 2001, 29 (1), 320-322.

93. Saferstein R., Hall P. An Introduction to Forensic Science. Criminalistics. Prentice Hall, Upper Saddle River, New Jersey 2011,108-112.

94. Saferstein R.E. Criminalistics: an introduction to forensic science, sixth ed., Prentice Hall, New Jersey, 1998.

95. Said T.M., Agarwal A, Zborowski M., Grunewald S., Glander H., Paasch U. Utility of Magnetic Cell Separation as a Molecular Sperm Preparation Technique. Journal of andrology 2008, 29(2):134-142.

96. Sato, M., \& Sato, K. Maternal inheritance of mitochondrial DNA by diverse mechanisms to eliminate paternal mitochondrial DNA. Biochimica et Biophysica Acta - Molecular Cell Research, 2013, 1833(8), 1979-1984.

97. Scientific Opinion on the toxicological evaluation of phenol. EFSA Journal 2013;11(4):3189, 44 pp.

98. Skoog, D.A., Holler, F.J., Crouch, S. R., Instrumental Analysis. Cengage Learning: 2007

99. Smejkal, G. B., Witzmann, F. A., Ringham, H., Small, D., Chase, S. F., Behnke, J., \& Ting, E. Sample preparation for two-dimensional gel electrophoresis using pressure cycling technology. Analytical Biochemistry. 2007, 363(2), 309-311.

100. Smejkal, G.B.; Robinson, M.H.; Lawrence, N.P.; Tao, F.; Saravis, C.A.; Schumacher, R.T. Increased Protein Yields from Escherichia coli Using PressureCycling Technology. J Biomol Tech 2006, 17(2): 173-175.

101. Smith L. M., Burgoyne L. A. Collecting, archiving and processing DNA from wildlife samples using FTA® databasing paper. BMC Ecology 2004, 4 (1), 4.

102. Song, Y., Zhu, Z., An, Y., Zhang, W., Zhang, H., Liu, D., Yang, C. J. Selection of DNA Aptamers against Epithelial Cell Adhesion Molecule for Cancer Cell Imaging and Circulating Tumor Cell Capture. 2013

103. Swander, C. J., \& Stites, J. G. Evaluation of the ABAcard HemaTrace for the forensic identification of human blood, 1-5. 
104. Thacker C., Phillips C. P., Syndercombe-Court D. Use of FTA cards in small volume PCR reactions. Progress in Forensic Genetics 8 1999, 2000, 8, 473-475

105. Thompson R., Zoppis S., McCord B. An overview of DNA typing methods for human identification: past, present, and future. DNA Electrophoresis Protocols for Forensic Genetics. Humana Press 2012, 3-16.

106. Tilstone, W. J., Savage, K. A., Clark, L. A. Forensic science: An encyclopedia of history, methods, and techniques. ABC-CLIO: 2006.

107. Valgren, C., \& Edenberger, E. Evaluation of the Differex TM System. $2008,1,78-79$.

108. Valls, C., Rojas, C., Pujadas, G., Garcia-Vallve, S., \& Mulero, M. Characterization of the activity and stability of amylase from saliva and detergent: Laboratory practicals for studying the activity and stability of amylase from saliva and various commercial detergents. Biochemistry and Molecular Biology Education, 2012, 40(4), 254-265.

109. Virkler K., Lednev I.K. Analysis of Body Fluids for Forensic Purposes: From Laboratory Testing to Non-Destructive Rapid Confirmatory Identification at a Crime Scene. Forensic Science International 2009, 188 (1-3), 1-17.

110. Voorhees J. C., Ferrance J. P., Landers J. P. Enhanced elution of sperm from cotton swabs via enzymatic digestion for rape kit analysis. Journal of Forensic Sciences. 2006, 51 (3), 574-579.

111. Vuichard S., Borer U., Bottinelli M., Cossu C., Malik N., Meier V., Gehrig C., Sulzer A, Morerod M., Castella V. Differential DNA extraction of challenging simulated sexual assault samples: a Swiss collaborative study. Investigative Genetics 2011, 2 (1), 11.

112. Walker, J. A.; Hedges, D. J.; Perodeau, B. P.; Landry, K. E.; Stoilova, N.; Laborde, M. E.; Shewale, J.; Sinha, S. K.; Batzer, M. A., Multiplex polymerase chain reaction for simultaneous quantitation of human nuclear, mitochondrial, and male Ychromosome DNA: application in human identification. Analytical Biochemistry 2005, 337 (1), 89-97.

113. Weaver, R., Molecular Biology. McGraw-Hill: 2011.

114. Willard J.M., Lee D.A., Holland M.M. Recovery of DNA for PCR amplification from blood and forensic samples using a chelating resin. Methods of Molecular Biology 1998, 98: 9-18

115. Witt S., Neumann J., Zierdt H., Gébel G., Röscheisen C. Establishing a novel automated magnetic bead-based method for the extraction of DNA from a variety of forensic samples. Forensic Science International: Genetics 2012, 6(5), 539-547. 
116. Xu, K., Poe, B. L., Lounsbury, J. a, \& Landers, P. (2012). Bead-Assisted Acoustic Differential Extraction of Sperm Cells in Dilute Samples for Potential Forensic Analyses. 2012, 3, 130-132.

117. Yamada M., Nakashima M., Seki M. Pinched flow fractionation: continuous size separation of particles utilizing a laminar flow profile in a pinched microchannel. Anal Chem. 2004, 76(18):5465-71.

118. Zhao X.C., Wang L., Sun J., Jiang B.W., Zhang E.L., et al. Isolating Sperm from Cell Mixtures Using Magnetic Beads Coupled with an Anti-PH-20 Antibody for Forensic DNA Analysis. PLoS ONE 2016. 11(7): e0159401

119. Untested Evidence in Sexual Assault Cases. (2016, March 3). Retrieved September 13, 2016, from http://www.nij.gov/topics/lawenforcement/investigations/sexual-assault/Pages/untested-sexual-assault.aspx

120. Higgins, D., Kaidonis, J., Townsend, G. \& Austin, J. J. Evaluation of carrier RNA and low volume demineralization for recovery of nuclear DNA from human teeth. Forensic Sci. Med. Pathol. 2014, 10, 56-61 (2014). 


\section{APPENDIX}

\section{Protocol: Pressure Cycling Technology (PCT) and Alkaline Lysis Differential Extraction Protocol}

$\underline{\text { Reagents }}$

$0.05 \mathrm{~N} \mathrm{NaOH}$

$0.4 \mathrm{~N} \mathrm{NaOH}$

$1 \mathrm{M} \mathrm{HCl}$

\section{$\underline{\text { Procedure }}$}

1) Cut the swab with sterile scissors and place it in a FT500-ND PULSETM tube.

2) Add $800 \mu \mathrm{L}$ of $0.05 \mathrm{~N} \mathrm{NaOH}$ to the swab sample.

3) Insert the PULSE tube into the Barocycler ${ }^{\circledR}$ instrument.

4) Set the Barocycler ${ }^{\circledR}$ to $20 \mathrm{k}$ psi target pressure for 10 cycles. Set T1 (holding time at ambient pressure) to 15 seconds and T2 (holding time at target pressure) to 15 seconds.

5) Transfer supernatant to a $2 \mathrm{~mL}$ microcentrifuge tube and place the swab in a spin basket with sterilized tweezers. Place spin basket back in tube and spin in centrifuge for 5 minutes at $13000 \mathrm{rpm}$.

6) Save the sample in the tube as the first epithelial fraction.

7) Place the swab in a new FT500-ND PULSE ${ }^{\mathrm{TM}}$ tube and insert the tube into Barocycler ${ }^{\circledR}$ instrument for another run.

8) Transfer supernatant to a $2 \mathrm{~mL}$ microcentrifuge tube and place the swab in a spin basket with sterilized tweezers. Place spin basket back in tube and spin in centrifuge for 5 minutes at $13000 \mathrm{rpm}$.

9) Save the sample in the tube as the second epithelial fraction.

10) Place the swab in a $1.5 \mathrm{~mL}$ microcentrifuge tube.

11) Add $400 \mu \mathrm{L}$ of $0.4 \mathrm{~N} \mathrm{NaOH}$ to the swab sample.

12) Incubate the sample in a $95^{\circ} \mathrm{C}$ water bath for 5 minutes. 
13) Place the swab in a spin basket with sterilized tweezers. Place spin basket back in tube and spin in centrifuge for 10 minutes at $13000 \mathrm{rpm}$.

14) Discard the swab and save the sperm fraction.

15) Neutralize the sperm fraction with $160 \mu \mathrm{L}$ of $1 \mathrm{M} \mathrm{HCl}$.

16) Purify with Biorobot EZ1 or Phenol/Chloroform/Isoamyl Alcohol (25:24:1) (PCIA).

\section{Protocol: Immunomagnetic Cell Capture Pretreatment for Differential Extraction}

\section{$\underline{\text { Reagents }}$}

Stemcell Technologies EasySep ${ }^{\mathrm{TM}}$ Human EpCAM Positive Selection Kit

- Human EpCAM Positive Selection Cocktail

- Magnetic Nanoparticles

\section{RoboSep ${ }^{\mathrm{TM}}$ Buffer}

$\underline{\text { Procedure }}$

1) Place swab in $5 \mathrm{~mL}$ BD Falcon ${ }^{\mathrm{TM}}$ tube and add $500 \mu \mathrm{L}$ of RoboSep ${ }^{\mathrm{TM}}$ Buffer.

2) Add $50 \mu \mathrm{L}$ of Human EpCAM Positive Selection Cocktail and mix well.

3) Incubate for 20 minutes on ice.

4) Gently re-suspend Magnetic Nanoparticles with pipet.

5) Add $25 \mu \mathrm{L}$ of Magnetic Nanoparticles and mix well.

6) Incubate for 15 minutes on ice.

7) Add $2 \mathrm{~mL}$ of RoboSep ${ }^{\mathrm{TM}}$ Buffer and mix well.

8) Place $5 \mathrm{~mL}$ BD Falcon ${ }^{\mathrm{TM}}$ tube in EasySep ${ }^{\mathrm{TM}}$ Magnet for 5 minutes.

9) Remove swab for extraction. 